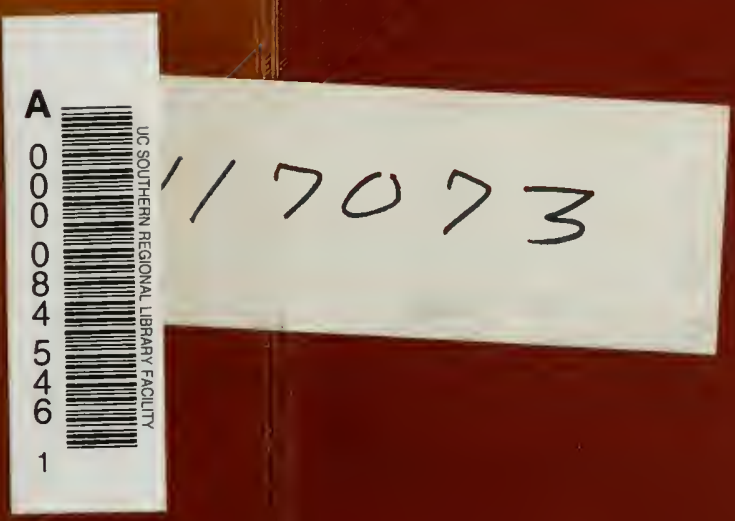




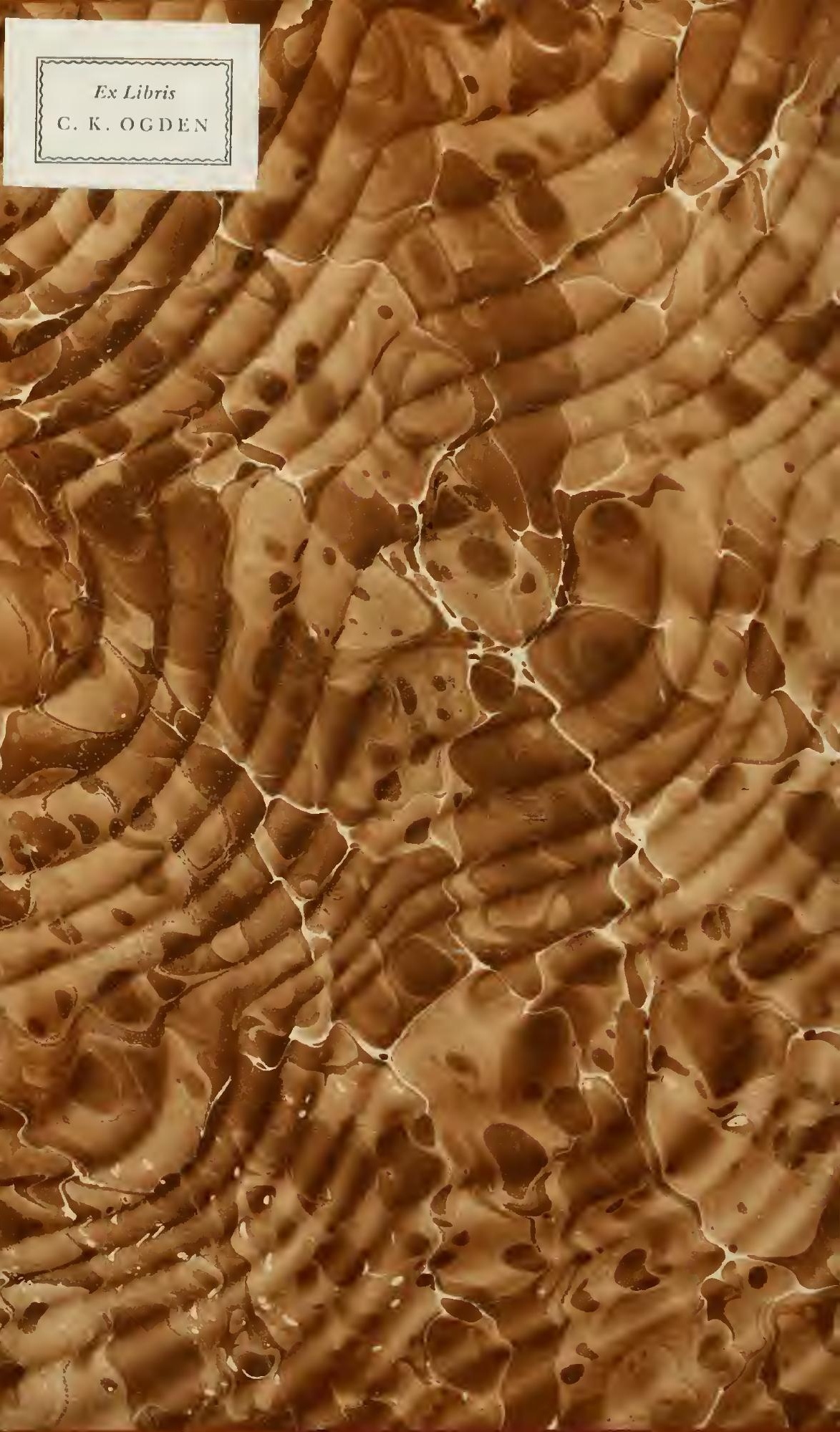




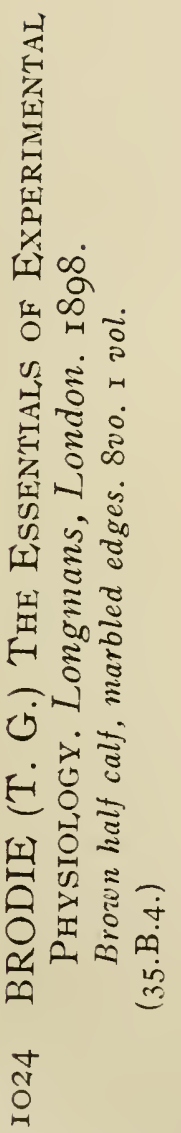





\section{EXPERIMENTAL PHYSIOLOGY}


A TEXT-BOOK OF CHEMICAL PHYSIOLOGY AND PATHO. LOGY. By W. D. HallibunTox, M.D., F.R.S., M.R.C.P., Professor of Physiology in King's College, London; Lecturer on Physiology at the Loudon School of Medicine for Women. With 104 Illistrations, 8vo. 28s.

ESSENTIALS OF CHEMICAL PHYSIOLOGY. By IV. D. HalliburTox, M.D., F.R.S., M.R.C.P., Professor of Physiology in King's College, London; Lecturer on Physiology at the London school of Medicine for Women. $8 \mathrm{vo}, 5 s$

*** This is a book suitable for medical students. It treats of the subject in the same way as Prof. Schäfre's 'Essentials' treats of Histology. It contaius a number of elementary and alvanced practical lessolis, followed in each ease by a brief descriptive account of the facts related to the excreises which are intendied to be performed by each member of the class.

AN INTRODUCTION TO HUMAN PHYSIOLOGY. By Adgustes D. WALLfi, MI.D., Lecturer on Physiology at St. Mary's Hospital Medical School, London; late External Examiner at the Victorian Universits. Third Edition, Revised. With 314 Illustrations. 8vo. 18..

LECTURES ON PHYSIOLOGY. By Augustus D. Waller, M.D., Lectırer on Physiology at St. Mary's Hospital Medical School, London: late Exterual Examiner at the Victorian University.

First Scries. ON Aximal Electrictity. 8ro. 5s. net.

EXERCISES IN PRACTICAL PHYSIOLOGY. Part I. Elementary Physiological Chemistry. By Augustus D. WALlen and W. Letge SrMes. 8vo. 1s. net. Part II. in the press. Part III. Pliysiology of the Nervous System; Electro-Physiology. 8vo. 2s.6d. net.

THE ESSENTIALS OE HISTOLOGY. Descriptive and Practical. For the Use of Students. BF E. A. SCHÄFER, F.R.S.. Jodrell Professor of Physiology in University College, London ; Editor of the Histological portion of Quain's 'A natomy.' Illustrated by more than 300 Figures, many of which are new. Fonrtl Ellition, Revised and Enlarged. 8vo. 7s, 6il. (Interlea serl, 10s.)

LONGMANS, GREEN, \& CO., 39 Paternoster Row, London New York and Bombar. 


\section{THE ESSENTIALS}

$\mathrm{OF}$

\section{EXPERIMENTAL PHYSIOLOGY}

FOR THE USE OF STUDENTS

BY

T. G. BRODIE, M.D.

LECTURER ON PHYSIOLOGY, ST THOMAS'S HOSPITAL MEDICAL SCHOOI,

LONGMANS, GREEN, AND CO.

39 PATERNOSTER ROW, LONDON NEW YORK AND BOMBAY

1898 



\section{P R E F C E}

Iv writing this book my aim has been to give a short account of those experiments which can be carried out by students during classes, together with a selection of experiments suitable for class demonstrations. In the selection of the experiments I have been largely guided by the course of Advanced Practical Physiology given by Professor Halliburton at King's College, which was based upon the 'Syllabus of Lectures' published by Professor J. Burdon SANderson in 1879, though in several respects I have modified and added to this course.

The illustrations are for the most part new. For permission to reproduce several of the figures of instruments I wish to thank Professors McKendrick, Yeo, Halliburton, and Wallek. The source of these figures is indicated in each case. The reproductions of the tracings are all new and taken from tracings specially prepared for the purpose. With very few exceptions they are all reproduced the same size as the originals, so that the measurements indicated in the text directly apply to the figures.

Those modifications of many of the usual forms of apparatus figured in the text have been made for me by Mr. C. F. Palmen, and are especially designed for class work.

The plan of the book varies slightly from that adopted by Professor SchäFER and Professor Halliburton in their 'Essentials.' I have 
made use of three different types: a small type employed in describing apparatus or the method of carrying out an experiment; a medium type forming the main body of the text; and a heavy type used in the accounts of the nore fundamental experiments which are fitted for elementary classes. It seemed better to mark off these elementary experiments in this way rather than to separate the book into an clementary and an advanced course. The number of elementary experiments given is only small, and a detailed list of them will be found on p. xiv.

To Professor Halliburton and to Professor Schäfer my best thanks are due for the many suggestions and criticisms with which they have aided me during the preparation of the book. To Dr. A. E. Russelu, Medical Registrar, St. Thomas's Hospital, I am especially indebted for many valuable suggestions and alterations, and for his assistance in reading and correcting the proofs.

T. G. BRODIE.

St. Thomas's Hospital, December 1897. 


\section{CONTENTS}

CHAPTER

I. Some Physical Instruments in Constant Use in Piysiological ExpERIMENTS

II. Preparation of a Frog's Muscle. Its Response to Stimulation. The Graphic Method. . . . . . . . 16

III. A Single Contraction of a Frog's Muscle. Its Modification under Changes in the External Condtions . . . . 30

IV. Sumition of Muscular Contraction. Tetanus . . . . 56

V. Fatigue of Musche . . . . . . . . . . . 66

VI. The Tilichening of a Muscle ox Contraction. The Muscle Wave 71

VII. Independent Muscular Excitability. Excitation of Musche by the Cosstant Curient. Polanisation of Electrodes. . . 76

VIII. Some Experinents to Determine the Functions of Nerves • 85

IX. Exanination of the Frog's Healit. The First Stannius Ligature 97

X. The Action of Heat and Coli upon the Frog's Heart • . 112

XI. The Nerves of the Frog's Heart and Their Functions • . 120

XiI. ACtion of Drugs upon the Frog's Heart . . . . . . 130

XIII. Some Further Methoos for Examining the Activitx of the Frog's Heart . . . . . . . . . . 134

XiV. Demonstiation of the Movejents of the Mamialian Heart. The Candiograph . . . . . . . . . 138

XV. Sone Experinents in Electro-physiology • . • • . . 146

XVI. Sciledia of the Circulation. The Sphygdograph • . . 157

XViI. Demonstration of Blood Pressure and its Nervous Regulation 168

XVIII. The Kinney. Demonstration of an Oxcometer Experiment 189

XiX. Demonstration of the Nervous Regulation of Respiration. The Stethoneter and Pneunograpil . . . . . . 201

XX. Demonstration of the Secretion of Saliva frodi the SubhaxilLaky Gland of thF Dog . . . . . . . . 209

XXI. Reflex Action as Studied opon the Spinal Cond of the Frog 214

XXII. Sone Expeninents in the Pirysiology of the Exe. Acconnodation, Ophthalmoscopy, Colour Sense, Permetry ․ . 218 



\section{LIST OF ILLUSTRATIONS}

FIG.

Plate I. Record of a Series of Successive Twitches of a Hyoglossus Muscle Stinulatei, by Make Shocks, once EACH SECOND . . . . . . . .

Plate II. Record of the Bloon Piessure and Respiration in a Rabbit during Asphyxia . . . . . . , , 182

1. The Daniell Battery (McKendrick) . . . . . . Page 2

2. The Grenet Battery (McKendrick) . . . . . . . . 2

3. The Bunsen Battery (McKendrick) . . . . . . . . 3

4. The Grove Battert . . . . . . . . . . 3

5. The Leclanchћ Battery (McKennitck) . . . . . . 4

6. The Induction Coll (McKendrick) . . . . . . . . . 4

7. Diagray of the Currents Induced in the Secondary Coll . * 6

8. Arrangenext of Apparatus for Equalising the Make and Breat SHоскS . . . . . . . . . . . . 7

9. To Illustrate the Action of Neef's Hamuer . . . . . 8

10. To Illustrate the Action of Neef's Hamier with the Helaholtz Modification . . . . . . . . . . . . 9

11. Two Forms of Mercury $\mathrm{Key}$ | . . . . . . . . 10

12. Two Forms of Mercury $\mathrm{Key} j$
13. Simple Form of Spring $\mathrm{Key}$. . . . . . . . . . . 10

14. Two Forms of the DU Bors Key \} . . . . . . . . 11

15. Two Foris of the Du Bois Key
16. Plan of the Arrangement of the Du Bois Key as a Short-circuitING KeY . . . . . . . . . . . . . . 11

17. Arrayged as a Simple Break Kex. Not to be used after this Method in a Secondary Circuit . . . . . . . 11

18. Pohl's Commutator . . . . . . . . . . . 12

19. A Form of Cut-out Key . . . . . . . . . 13

20. Arrangement of Apparatus for Making Use of Single Induckd SноскS . . . . . . . . . . . . . 14

21. Arrangement of Apparatus to Show the Break Extra-current • 14

22. Lea Muscles of the Frog seen frou the Inver Side . • • . 17

23. Leg Muscles of the Frog seen from the Outer Side • . . 17

24. Gastrocnemius-sciatic Preparation . . . . . . . 18

25. Arranoment of Apparatus for Showing Mate Extra-current . 19

26. The Relations of the Hyoglossus in the Frog . . . . . 20

27. Two Records of the Vishations of a Tuning-fork Vibrating at the RAte of 10 PER SEC. . . . . . . . . . . 22

28. A Time-harker or Chronograph (McKendrick) • . . . . 23

29. A Spring Chronograph . . . . . . . . . . 21

30. Lower Part of Drum to show Method of Driving the Crinder. at Different Rates 


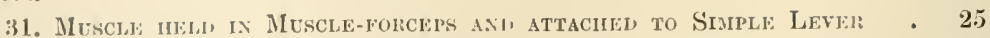

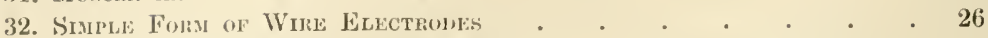

33. Heigits of Costractiox of a Muscle witil Different Strengths of Stтnul . . . . . . . . . . . . 27

34. Mamey's Fomir of Reconding Tambodi (Mchexinick) . . . . 28

35. A Second fonm of Reconing Tambul . . . . . . . 29

36. A BREAK KEY . . . . . . . . . . 30

37. Plin of the Arrangement of the Apparates for Recording a Simple Twitcil . . . . . . . . . 31

38. Isotonic Twitch of a Hroglossus Muscle . . . . . . 34

39. To Illustrate the Meaning of the Cuive of Fig. 38 . . . . 35

40. Sidple Tiwtch of a Gastrockeanus . . . . . . . 36

41. Twitch of a Hyoglossus recorded bi a Heavt Level . • . . 38

42. The Prixciple of the Isotonic Nethol . . . . . . . . . 39

43. The Principle of the Isometric Miethon . . . . . . 40

44. Muscle Attached to an Isodietric Letel . . . . . . 40

45. Timen Isometric Twitches mith Different Initial Texsions • . 41

46. Arraxaejext of Sinple Lever for Recording by the Method of

After-lOAD . . . . . . . . . . . 4 43

47. Twitches Takex under the Principle of After-loading . . . 44

48. Apparatus for Varisig the Temperatures of a Muscle by Immersiox 45

49. Twitches of a Hyoglossus at Different Temperatures . • . 46

50. Twitches of a Gastrocanemius at Different Temperatures . . . 47

51. Twitches of a Hyoglossus with Different Loads . . . . 48

52. Twitches of a Gastrocnemites with Different Loads . . . . 48

53. Two Twitches given by a Muscle Poisoned with Veratrine . $\quad 50$

54. Work-hiaray of a Gastrociemius for Single Twitches . . . 51

55. Simple Fori of Pexdulua Mrograph . . . . . . . . 53

56. Trigger Kex . . . . . . . . . . . . . . 54

57. Simple Twttch of Hyoglosses Muscle: recordey bi the Pfadulua

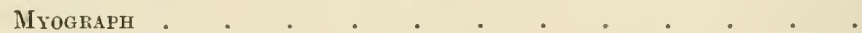

58. Arrangemext of Upper Part of Drem for applyixg Two Stimul to A Muscle

59. Effect of Two Successive Stimuli, with Gradualix Dimisishixg INtervals, upox a Gastrochemitus . . . . . . .

60. Node of Fitting up a Vibratixg Reed . . . . . . . 60

61. Reed arranged to Vibrate in a Horizontal Plane . . . . 61

62. The Gradual Production of Tetands as the Rate of Stimulation WAS INCREASED.

63. The Genesis of Tetanes with Slow Rotation of Recondng Surface

64. A Series of Twitches of the Hroglossus aiade to Coxtract every Half-Second to show the Alteration in the Twitch as the Muscle becomes Fatigued

65. Prolonged Tetanisation of a Hroglosstes showing Relaxation as the Moscle becaje Fatigued

66. Method of Recordng the Thichenitg of a Muscle as it Contracts

67. Curve I the Shortexing, axd Curve II the Thicienisg of a Semimembraxosus and Gracilis Preparation . . . . . . 72

68. Diagram to Illustrate Wave Mofemest . . . . . . 73 
FIG.

69. Apparatus for Recording tue Thickening of a Muscle at Two Points, for the purpose of Studying the Muscle Wave . . . . 74

70. The Thickening of a Muscle . . . . . . . . . . 75

71. Method of Studying Polar Excitation of a Muscle . . . . 80

72. Method of Arranging the Apparatus to Show Polarisation of ElecTRODES

73. Several Models of Unpolarisarle Electrodes (Waller) . •

74. Simple Form of Unpolarisable Electrodes . . . . . .

75. Plax of Apparatus for Studing the Chaxges of Excitability of Electrotonus

76. Diagram Indicating the Changes of Excitability of a Nerve in Electrotonus .

77. To Illustrate the Principle of the Moxochord

78. A Second Form of Monochond. . . . . . . . .

79. The Rheochord as Arranged for Varying the Direction and Strength of a Current throdgh a Nerve . . . . .

80. Arrangement of Apparatus for Studing the Velocity of a Nervous

IMPULSE . . . . . . . . . . . . .

81. Two Twitches of a Gastrocnemius when the Sciatic was Stimulated

82. Anterior and Posterior Surfaces of the Frog's Heart . .

83. Apparatus for Recording the Heatt Beat my the Suspexsion Method

84. Record of the Movenents of the Frog's Heart by the Suspension METHod .

85. Application of the First Stannius Ligature to the Frog's Heart

86. Electrical Stimulation of the Ventricle of the Frog's Heart in

Standstill dy the Stannius Ligature to Show the 'Staircase'

EFFECT .

87. A Simple Form of Flexible Electrodes

88. A Single Contraction of the Frog's Ventricle . . . . 107

89. A Single Contraction of the Frog's Ventricle • . . . . 108

90. A Repetition of the Tracing of Fig. 89 with a Slower Movement of the Recording Surface

91. The Effect of Two Successive Struul upon the Ventricle of the Frog's Heart .

92. Tetanisation of a Frog's Ventricle in Standstill by the Stannius

Ligature

93. Apparatus for Varying the Temperature of a Frog's Heatit .

94 a. Tracings Obtained by Imuersing an Excised Frog's Heart in

Diluted Blood at Different Temperatures . . . .

94 b. Tracings Obtaned by Imuersing an Excised Frog's Heart in

Diluted Bloon at Different Temperatures. . . . .

95. Single Contractions of the Frog's Ventricle at Various TejiperaTURES

96. Diagrals of the Variations of the Duration of a Single Vextricle Contraction at Different Temperatures. • . • . .

97. Diagrail of the Variations in Heigitit of Contraction of the Frog's Ventricle at Different Temperatures . • . . • . 118

98. To Show the Counse of the Vagus in the Frog . . . . 120

99. The Course of the Sympatiete in tile Frog . . . . . 121 
Fir.

100. Ehfects of Thtanisixa the Vagus wite Differext Strexctus of

Strmul • • • • • • • • • • . 123

101. The Effect of Tetanisation of the Vagus • • • • • 126

102. Strmulation of the Sympatietic . . • • • • • . 127

103. The Efrect of Muscarixe and Atropine on the Frog's Heart • 131

104. The Effect of Appling a Weak Solution of Nicotine directir to THE Heart. . .

105. Tracings lecorded by a Lever Resting upon a Frog's Heart . 134

106. Rox's Tonometer (Halimurton) . . . . . . . . 135

107. Schälen's Frog-heart Plethyshograpi • • • • • • • . 136

108. Frog-heart Plethysmograph by which the Pressure Changes can also be Recorded bx a Siall Manometer . . . . . . 137

109. A Simple Fory of Apparatus for Antificlal Respiration . • . 13!

110. Arraxgement of Levers for Recording the Movements of the Mammalin Heart by Attachisg Threads to the Auricle and Ventricle - RESPECTIVLY • • • • • • • • • • •

111. Tracing Obtained from the Rabbit's Heart, Ejploying the Levers OF FIG. 110 .

112. Result of the Stimulation of the Left Vagus . . . . .

113. Result of the Injection of 1 c.c. of a 4 per cent. Solution of Caffeine Citrate

114. The Cardiograph

115. A Cardiogray taken opon a Man

116. Kühne's Experiment of Contraction without Metals . . . . 148

117. Arrangement of Apparatus for Showing Secondary Contraction - 148

118. Side View of Galvanometer and Shunt, Lamp and Scale (Waller). 149

119. Course of Current through Galvaxometer (Waller) — . . 150

120. Puan of De Bois-Reymond's Method of Measuring the Musche Currents

121. Lippalaxy's Capillary Electrometer (Waller)

123. Arrangement of Apparatus to Show the Paradoxical Contraction . 156

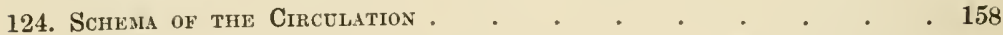

125. Apparatus for Studing the Passage of a Pulse Wave along an Elastic Tube

126. Marey's Sphrgiograph (Halliburton).

127. Diagram to Show the Arrangement of the Levers in Marey's

SPHYGMOGRAPH .

128. Sphyghogray Taken by Marey's Sphyghograph . . . . . 165

129. Richardon's Modification of Dudgeon's Sphygmograph a . . 166

130. Plai of the Levers in Dudgeon's Sphygnograph . . . . . 166

131. Two Sphyghograyis Taken by a Dudgen's Sphygmograph • . . 167

132. Arrangement of Apparatus for a Blood-pressure Experiment . . 169

133. Dissection of the Nerves of a Rabbit's Neck . • . . . 170

134. Two Forms of Cannulaz . . . . . . . . . . 170

135. Tracing of the Blood Pressure from a Rabbit taken bx the Mercury Manometer • • • • • • • • • . 172

136. Blood Pressure and Respmatory Tracing of a Curarised Cat under

Mоврна . . . . . . . . . . . 173 
FIG.

137. Stimulation of the Depressor Nerve in a Rabiti * . . . 174

138. Stimulation of the Cextral End of the drined Sciatic . . . 176

139. Pressor Effect Produced ry Struilating the Central Exi or the

Sciatic of a Curarised Cat under Morphis . . . . . . 177

140. Stimulation of tie Periphenal End of the Left Vages iN a Rabbit 178

141. Two Successive Stimulations of the Peripheral Fnd of the Right

Vagus with the Same Strength of Strimlus . . . . . . 179

142. Stimulation of the Cextral Exi of the Left Vagus in a Rabit . 180

143. Effect of an Injection of Nicotine upon the Brood Pressure . 181

144. To Illustrate the Ixertia of a Mercury Manometer . . . . 183

145. Fick's C-Spring Manometer (Yeo) . . . . . . . . $\quad$. 183

146. Tracing by Fich's Mixometer. . . . . . . . . . . 184

147. Hürthle's Manometer . . . . . . . . . . . . . 184

148. Tracings of Blood Pressure of the Rabitit ry Hürthle's Manojeter 185

149. Strifulatiox of the Perifheral Exd of the Vagos . . . 186

150. Noralal Brood Pressure axt Respimition . . . . . 187

151. Lodwig's Stroiruhr . . . . . . . . . . . . . . . 187

152. Two Sizes of Roy's KidNey ONcometeri . . . . . . . 190

153. To Illustrate the Prixciple of Roy's Oxconeter _ . . . 190

154. The Oxcograph . . . . . . . . . . . . . . 191

155. AN Air Oxcometer Fol: The KinNey _ . . . . . . . . 191

156. Simultaneous Tracing of the Volume Changes of the Kidney ani) of the Cafiotid Bloot Pressure in a Dog . . . . . . 194

157. Kidney Volume and Blood Pressure in a Dog • . . . . 196

158. Effect of Caffeine upox the Kidney Voluje and Bloon Phessure 197

159. Effect of Nedrine upox the Kinney Voldale dxd Blood Pressure 199

160. Alteratiox in Respiration on Strafulation of the Superior LaryxGEAL NERVE

161. EFfect upon Respiration of Stimulation of the Glossophariggeal Nirve.

162. Result of Section of the Vagus, the other Nerve having beex Prleviously Diviled . . . . . . . . . .

163. Stimulation of the Cextral Exd of the Vagus, both Vagi having BEEN DIVIDED

164. Stimuration of the Central Eni of the Vagus, both Vagi havisg BEEN DIVIDED .

165. Marky's Pneumograph (McKendrick)

166. SANDERson's Stethojeter .

167. Mode of Applying the Stethometer to Recond Changes 1N Transversse Dianeter of the Cines't

168. Record of Chasges in thl Transverse Diajeter of thi: Thorax during Respiration (Man)

169. The Relation of the Veins to the Subajaxiluary Gland in the Dog

170. Relations of the Duct axd Neives of the Subuaxiliary Gland in THE Dog

171. The Pilamoscole (McKexdricii).

172. The Rephected Images as seen in the Phajoscor'e . . . . 220

173. To Illustrat'e Scheiner's Expeithext . . . . . . . . 221 
NIT:

174. The Course of the Ligit in the Indinect Method of Euploying THE: OPMTIIALMOSCOPE . • • • • • • • • . .

175. The Course of tuie Ligit in Examining the Eye by the Direct METHOD • . . . . . . • • . . 223

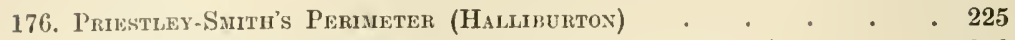

177. A Penimetric Chart for tue Right Eye (Halliburton) • • . 226

The experiments and descriptions of apparatus for elementary classes will be found in the following positions, and are indicated by being printed in heavy type :-

The Induction Coll

PAGE

Description of the More Important Keys . . . . . . . . 10

The Nerve-muscle Preparation . . . . . . . . . . 16

The Hyoglossus Preparation . . . . . . . . . . . 20

The Simple Lever. . . . . . . . . . . . 25

Minimal and Maxianal Excitation . . . . . . . . . 26

The Sinple Muscle Curve . . . . . . . . . . . 30

The Work Perforded during a Twitcil . . . . . . . . . . 50

Tetanisation of a Muscle . . . . . . . . . . . . 65

Thickening of a Muscle durisg a Twitch . . . . . . . 71

Independent Muscular Excitability . . . . . . . . . . 76

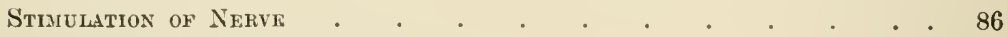

Record of the Beat of the Frog's Heart . . . . . . . . . 97

Excision of the Frog's Heart . . . . . . . . . 101

Action of the Vagus upon the Frog's Heart . . . . . . 120

Reflex Action Studied on the Frog . . . . . . . . 214 


\title{
THE ESSENTIALS
}

OF

\section{EXPERIMENTAL PHYSIOLOGY}

\author{
CHAPTER I \\ SOME PHYSICAL INSTRUMENTS IN CONSTANT USE IN \\ PHYSIOLOGICAL EXPERIMENTS
}

BEFORE undertaking any purely physiological experiments it is necessary to understand the construction and mode of working of certain pieces of physical apparatus which are in constant use ; such, for instance, as batteries, induction coils, keys, \&c.

The Daniell's Element (fig. 1) is in very general use, on account of the constancy of the current it yields. It consists of an outer vessel of glass or glazed earthenware, in which is placed a cylinder of copper open at both ends. Within the copper cylinder is a porous pot, and within this is a roll of zinc. The outer vessel is filled with a saturated solution of sulphate of copper, and an excess of the crystals is kept in the solution. The porous pot is filled with dilute sulphuric acid ( 1 to 5 of water). Connections are taken from the copper and zinc cylinders. The positive pole of the battery is the copper, the negative the zinc. To prevent local action the zine cylinder is previously thoroughly amalgamated by first cleaning its surface with dilute sulphuric acid, and then rubbing metallic mercury well over its surface with a piece of cloth dipped in the acid. When in action the chemical changes in the battery are, solution of zinc and formation of $\mathrm{ZnSO}_{4}$ at the zinc plate, and decomposition of the $\mathrm{CuSO}_{4}$, by the hydrogen appearing at the copper plate to form $\mathrm{H}_{2} \mathrm{SO}_{4}$ and metallic $\mathrm{Cu}$, which latter is deposited on the copper surface. The E.M.F. (electromotive force) of the battery is 1.072 volts. 
Grenet's Battery (fig. 2) is a single fluid battery. It consists of an amalgamated zinc plate fixed between two carbon plates $\mathrm{K}, \mathrm{K}$. The zinc plate is fixcd above to a rod $\mathrm{B}$, by means of which it can be lifted from the fluid. The two carlon plates are connected to the binding screw, $\mathrm{B}$, which is therefore the positive pole; the zinc is

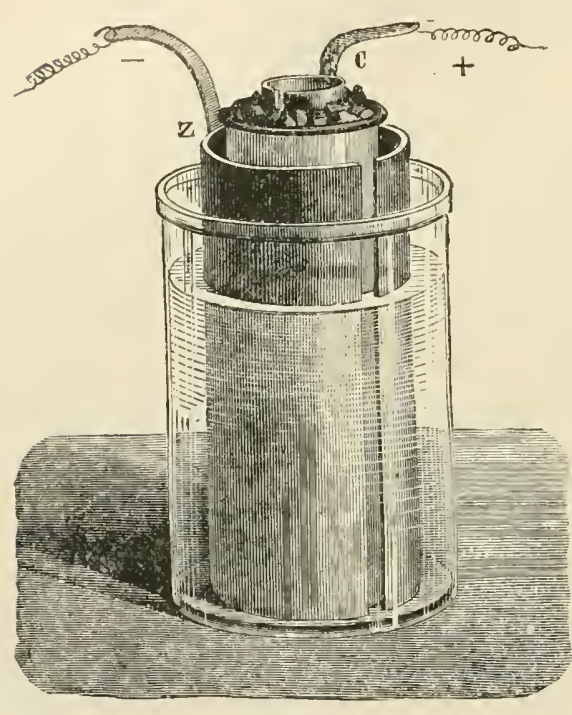

F'ig. 1.-The Daxiell Batters.

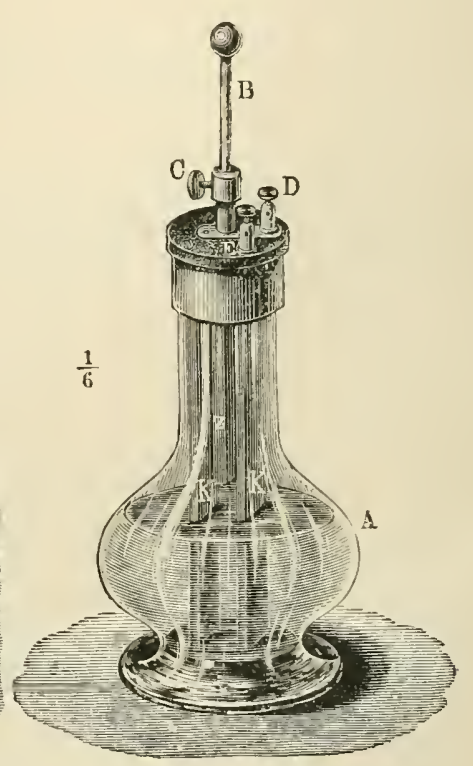

Fig. 2.-The Grenet Batteri.

connected to D. The fluid is made by adding four parts of a 10 per $^{2}$ cent. solution of potassium bichromate to one of sulphuric acid. In action the zinc is dissolved, and the hydrogen set free at the carbon plates is oxidised by the bichromate and thus removed. When freshly made the battery has an E.M.F. slightly above 2 volts, but rapidly falls until it reaches about 1.8 volts.

A Bunsen Battery (fig. 3) consists of an outer earthenware pot in which is placed a zinc cylinder. Inside this is a porous pot carrying a square block of carbon, $c$. The wire connections are made to the carbon, the positive pole, and to the zinc, the negative pole. The porous pot is filled with strong nitric acid, and the fluid surrounding the amalgamated zinc is dilute sulphuric acid ( 1 to 7 ). The $\mathrm{SO}_{4}$ appearing at the zinc plate when the battery is in action dissolves the zine to form $\mathrm{ZnSO}_{4}$, and the $\mathrm{H}_{2}$ appearing simultaneously at the 
carbon pole is oxidised into $\mathrm{H}_{2} \mathrm{O}$ by the nitric acid. The E.M.F. of the battery is 1.9 volts.

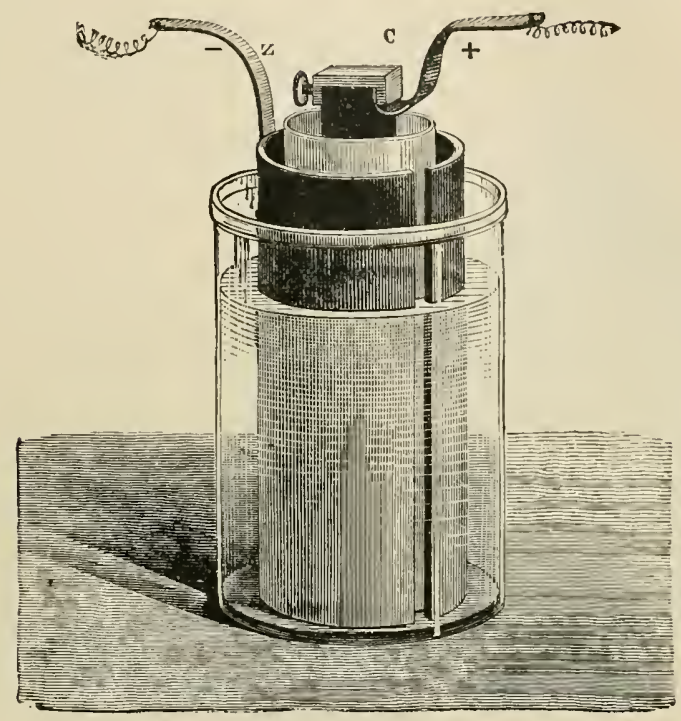

Fig. 3. - The Bunsen Battery, (McKendiek.)

The Grove Battery (fig. 4) is similar to the Bunsen battery, but the carbon is replaced by a sheet of platinum. Its E.M.F. is 1.96 volts.

The Leclanché Battery (fig. 5) consists of a glass jar containing a saturated solution of ammonium chloride into which an amalgamated zinc rod dips. This forms the negative terminal. The positive consists of a carbon plate fitted into a porous pot packed with small pieces of carbon mixed with manganese dioxide. The porous pot is then filled up with the ammonium chloride solution. Its E.M.F. when freshly prepared is 1.48 volts. It has the disadvantage that it tends to polarise rather quickly, and

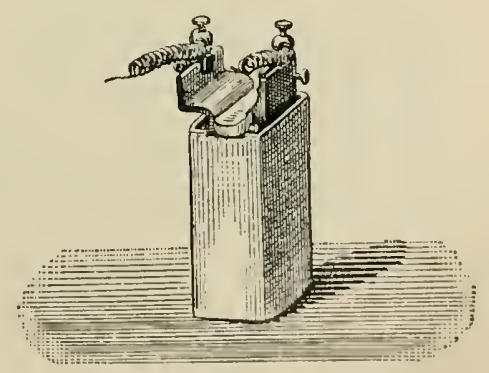

Fig. 4.-The Grove Battery. is therefore only used when a current is required for short periods of time. It is very convenient, as it does not fume; there are no acids to be spilt, and it does not require much attention. 
Dry Batteries. These are of very great convenience in that they are always ready for use, do not give off fumes, and contain no fluid

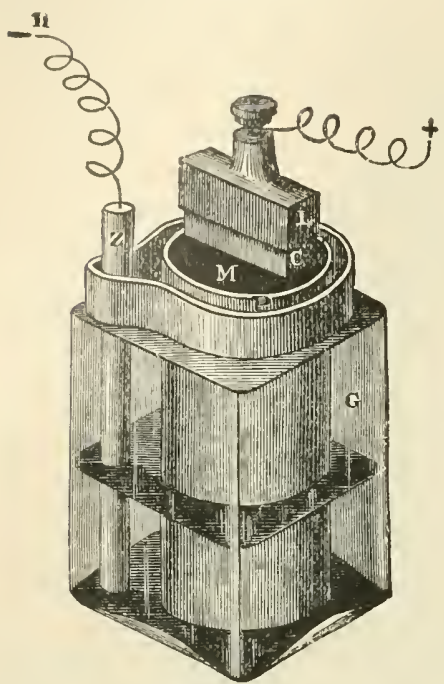

Fig. 5.-The Leclanché Battery. to be spilt. One of the most satisfactory of these is the Obach dry battery, manufactured by Siemens. In principle, they are usually modified Leclanché cells.

\section{THE INDUCTION COIL}

The form of induction coil usually employed by physiologists is Du BoisReymond's sledge inductorium (fig. 6). It consists of a coil, $a$, of fairly stout insulated copper wire wound on a wooden reel in the centre of which is a core of soft iron wires, $c$. The number of turns of wire in this, the PRIMARY COIL, varies in different instruments from 200 to 500 or more. The ends of the wire of the primary coil are connected to the two binding screws $f$ and $h$. A second coil of much finer wire is wound round a large wooden bobbin, the whole forming the SECONDARY COIL, $b$. This is fixed to a wooden foot sliding in a

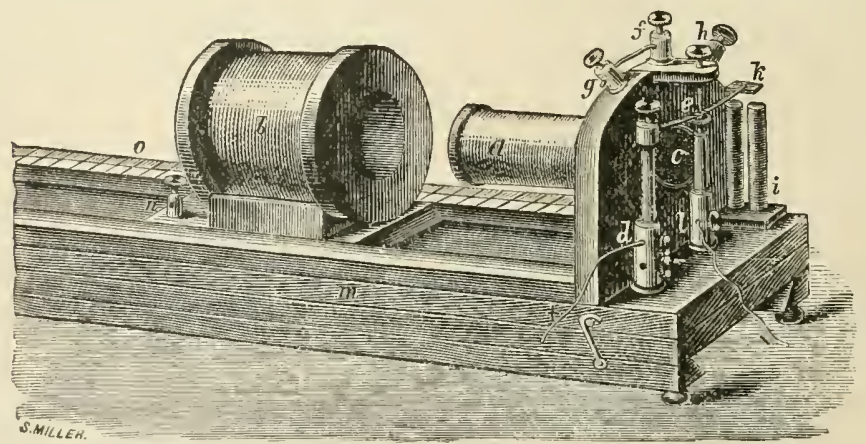

Fig. 6.--The Indoction Coll. (McKendick.)

grooved base, $m$, and the central cavity in the wooden bobbin is of such a size that the secondary coil may be pushed home so as to completely cover the primary coil $a$. The terminations of the wire of the secondary coil are connected to two binding screws, only one of which, $n$, 
can be seen in the figure. The number of turns of wire in this coil is 5.000 or more. The turns of wire in each coil are carefully insulated from each other.

The action of the coil depends upon the fact that if the strength of a current running along a wire be altered, an induced current is set up in a second wire placed near to it.

The F.M.F. of the induced current depends upon several factors :

1. It is directly proportional to the intensity of the current change in the first wire.

2. It is directly proportional to the rate of change of the inducing current.

3. It is inversely proportional to the distance between the two wires.

4. It varies with the angle between the two wires, the maximum effect being produced when the wires are parallel to each other, and no effect when they are at right angles to each other.

5. The strength of the induced current may be increased by concentrating the force of the magnetic field; as, for instance, by placing a coil of soft iron wires in the interior of the primary coil.

Some or all of these various factor's are utilised in the production of an induced current for physiological purposes; but as the induced current produced by the induction of one wire upon one other is very small, the induction coil forms a very convenient means by which these weak induced shocks may be multiplied and added to one another. By taking a large number of turns of wire in each coil the effect is greatly increased, because each turn of the primary coil induces a current in each of the turns of the secondary, and all these small effects are added together to produce a single greatly increased effect. We have seen that an induced current is only produced in the secondary coil during a change in the strength of the current in the primary, so that if that change be effected instantaneously, as in breaking the current, the induced current is also instantaneous. The direction of the induced current is such as to tend to oppose the new change, so that if a current be suddenly sent into the primary coil, round which it runs in the direction of the hands of a watch, the induced current in the secondary coil passes along its turns in the reverse direction, i.e. against the direction of the hands of a watch. Conversely, on suddenly breaking the primary eurrent, the induced current is in the same direction as that in the primary.

In a consideration of the action of the induction coil, there is a further point of some considerable importance, for just as the wires of the primary can react upon the wires of the secondary coil, so car 
each turn of the primary induce currents in each neighbouring turn of the coil. If we consider two neighbouring turns when the current is suddenly increased, the increase in the one wire will induce a current in the second, and this induced current will be in the reverse direction to that of the main current, and as the direction of the current in two neighbouring turns is the same it tends to diminish the amount of the increase in the second wire. As the duration of this induced current is very short its effect is soon exhausted, but not before it has produced the result that more time is required for the current to reach its full strength than would have been the case if the wire had been perfectly straight. On breaking the circuit the circuit of the primary is broken, so that no induction currents can be set up in the primary. The fall in potential is therefore instantaneous. These effects are diagrammatically represented in fig. 7. In this

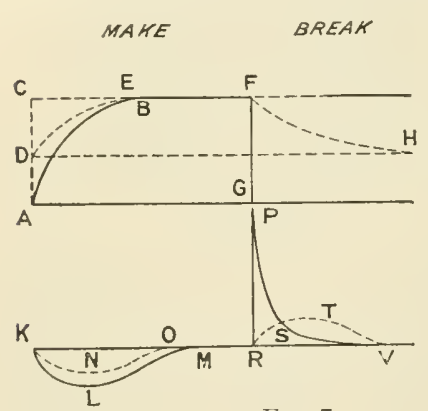

Fig. 7. figure, lines written horizontally indicate time, and vertical lines strength of current. At the instant $A$ a current whose amount is represented by the vertical line $\mathrm{A} \mathrm{C}$ is suddenly thrown into the primary, but instead of instantly reaching its full intensity, when the course of events would be represented by the line $\mathrm{A}$ c, time is occupied before it attains its full strength. Thus the gradual rise of strength of the current is represented by the curved line $\mathrm{A}$ B. At the instant $\mathrm{G}$ the current is broken, and there occurs an instantaneous fall in strength to zero, which is thus represented by the line F G. The induction effect produced in the primary on making the circuit is spoken of as the make extra-current. The result of this upon the current induced in the secondary coil is of very great importance. One of the chief factors varying the intensity of the induced current is the rate at which the change is effected, and as the make takes an appreciable time while the break is instantaneous, it follows that the induced secondary current at make is of less E.M.F. than that at break, but lasts longer. This is indicated in the lower half of fig. 7 . The line $\mathrm{K} \mathrm{r}$ indicates zero current, and the curved line $\mathrm{k} \mathrm{L} \mathrm{i}$ the current induced in the secondary by the change of current A $\mathrm{B}$ in the primary. The intensity of the change at any instant is indicated by the vertical height of the curve for that instant, and is drawn below the line $\mathrm{K} \mathrm{II}$, 
because the current is in the reverse direction to that of $A \mathrm{~B}$. The line R P S indicates the current induced in the secondary by the sudden change $\mathrm{F} \mathrm{G}$ in the primary: it is above the line $\mathrm{K} \mathrm{R}$ because it is in the same direction as the inducing current, and is of greater height than that representing the current induced on make. Von Helmholtz showed how we might approximately equalise the two induced shocks by the introduction of a deriving circuit into that through the primary. Fig. 8 shows how to arrange the apparatus to demonstrate this. A battery is connected to the two terminals of the primary coil, and to these are two further wires connected to a key and forming the derived circuit. It is seen that there is always some current passing through the primary both when the key is open and closed. When the key is closed the current from the

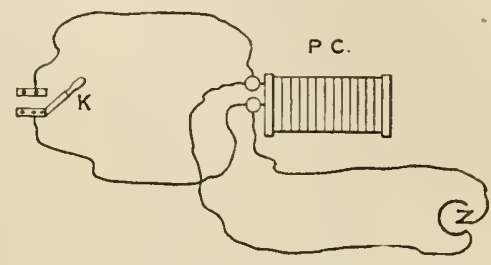

Firi, 8.-Amrangement of Apparatus For EQUAlising THE MAKE AND Break Shocks. battery on reaching the first terminal of the coil divides into two parts, one passing throngh the coil, the other through the deriving circuit. The amount of current passing throngh either circuit is inversely proportional to the total resistance in that circuit. If then the resistance of the deriving circuit be small in comparison with that of the coil, only a small proportion of the total current passes through the coil. On opening the key, the whole of the current is thrown through the coil and, as previously explained, an extra-current is produced which for a time delays the establishment of the current to its full intensity. On closing the key, there is a fall of current which produces an extra-current running in the same direction as that of the main current; and as the circuit through the primary is still closed, this extra-current can act in delaying the fall of strength of the current. The result is that the current induced in the secondary is considerably diminished and made approximately equal to that of the make. These results are indicated in the diagrams of fig. 7. The current passing through the primary when the key of the derived circuit is closed is indicated by $\mathrm{A} \mathrm{D}$. On opening the key the current rises in value to $\mathrm{A} \mathrm{C}$, but its course is delayed and takes the course represented by the dotted line $\mathrm{DE}$. If the key be opened at $\mathrm{F}$, the fall in strength to the line $\mathrm{D}$ н is not instantaneous, but takes time and is represented by the curved line $\mathrm{FH}$. The effects on the induced currents in the secondary circuit are represented by the interrupted lines $\mathrm{K} \mathrm{N} \mathrm{O}$ and $\mathrm{k} \mathrm{T} \mathrm{V}$ respectively.

For very many purposes it is essential to have a rapid series of 
induction shocks, which call of course be obtained by a rapid make and break of the circuit through the primary. To obtain this in an automatic way the induction coil is always fitted with an arrangement termed the NEEF'S HAMMER. This is represented in fig. 6, and consists of a pillar $d$ carrying a steel spring to which is attached an iron armature $k$. In the centre of this spring is a small platinum

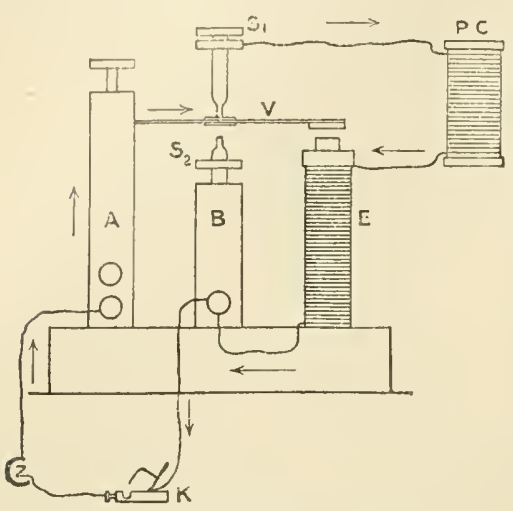

Fig. 9.-To Illestrate the Action of NeEF's Hamuer. plate for making contact with the platinum point of a screw adjustable in a brass plate connected to the binding screw $f$, and therefore with one terminal of the primary. Fixed under $k$ is a double electromagnet $i$, o:1e end of the wire of which is connected to $h$, the second terminal of the primary coil, and the other end to a central pillar $l$. The mode of action is illustrated by fig. 9. A battery is connected by one pole to the pillar $\mathrm{A}$ and by the other to the pillar $\mathrm{r}$, using a mercury key $\mathrm{k}$. If the platinum point of the screw $s_{1}$ be in contact with the platinum plate on the upper surface of the spring $r$, then on closing the key $K$ the circuit is closed, and if we suppose the positive pole of the battery to be in connection with the pillar a the course of the current is from the battery to $\mathrm{A}$, then along the spring $\mathrm{v}$ to the screw $\mathrm{s}_{1}$, thence through the primary coil to the electromagnet, and from this to the second pillar $\mathrm{B}$, and so through the key $\mathrm{K}$ back to the battery.

As soon as the circuit is thus closed the electromagnet acts upon the armature and pulls down the spring $\mathrm{v}$, thereby separating the two platinum surfaces. The current is at once broken, and the electromagnet therefore ceases to attract the armature, which is carried up by the spring $\mathrm{v}$; a new contact is thus made by the platinum surfaces, and the whole cycle of events is repeated. In this way the circuit through the primary is made and broken automatically at a rate which depends solely upon the rate of oscillation of the steel spring v. At each make and at each break of the circuit induced currents are produced in the secondary circuit, which, as previously explained, are of very unequal intensities.

Von Helmholtz showed how the Neef's hammer might be arranged to give shocks of about the same intensity. All that is necessary is to 
connect the pillar $d$ (fig. 6) with the binding screw $f$ by a stout wire and screw up the screws $s_{1}$ and $s_{2}$ (fig. 10) until $s_{1}$ is removed from contact with the spring $\mathrm{v}$, and $\mathrm{s}_{2}$ lies just below it, but not touching it. Fig. 10 illustrates the action of the hammer with this arrangement. The connections to the battery remain the same. On closing the key $\pi$ the path of the current is now from the battery to the pillar $\mathrm{A}$, and from this by the stout wire to the screw $\mathrm{s}_{1}$, and thence to the primar'y coil P C. From the primary coil it passes to the electromagnet $\mathrm{E}$, thence to the pillar $\mathrm{B}$, and so through the key $\mathrm{K}$ back to the battery. Immediately the current is closed the electromagnet attracts the armature of the spring $\mathrm{v}$, and as it pulls it down brings the platinum plate on its lower surface into contact with the platinum point of the screw $\mathrm{s}_{2}$, the result of which is that the derived circuit from the pillar $\mathrm{A}$ through the spring $\mathrm{v}$ to the pillar $B$ is closed. The current is now divided, and instead of all passing through the primary coil and electromagnet

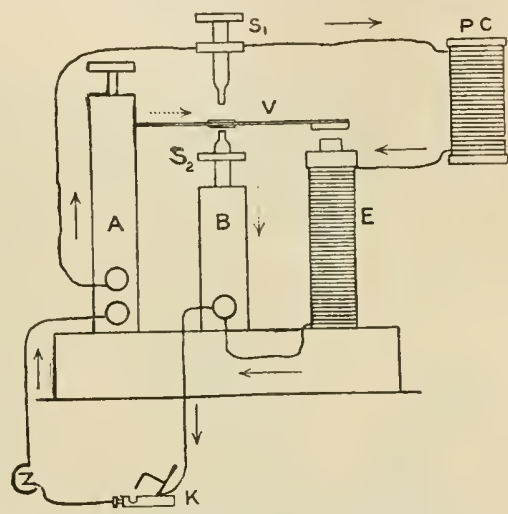

Fig, 10.-To Illustrate the Action of NeEf's Hamier with the Helmholtz Modification. most travels through the derived circuit, because the resistance of this is much less than that of the coil and electromagnet. The current of the electromagnet becoming so much weaker is now unable to resist the upward pull of the spring $\mathrm{r}$, which therefore recoils, and thus breaks its contact with the screw $\mathrm{s}_{2}$. The derived circuit is broken and the whole current again sent through the coil, the cycle is repeated, and so on continuously.

Just as in the previously described case where a simple derived circuit was used to equalise the make and break shocks this arrangement attains the same end, and is to be used when it is necessary that the two shocks should be nearly equal.

One of the great conveniences of the sledge inductorium is the ready manner in which the strength of the induced shock can be varied by simply altering the distance of the secondary coil from the primary. It must, however, be remembered that the strength of the induced current is by no means inversely proportional to the distance of the secondary coil from the primary, but that the strength of the induced current increases at a far greater rate than the diminution of 
distance between the two coils. The value of the induced current may be determined empirically by use of the galvanometer. Some forms of coil are already graduated in this manner.

Another plan which is at times adopted for varying the strength of the induced current is to have the secondary coil so fitted that it can be rotated and its long axis set at any angle to the axis of the primary. The induced current, with a fixed alteration in the primary, is then proportional to the cosine of the angle between the two axes of the coils.

\section{SOME FORMS OF KEYS FOR OPENING AND CLOSING A CIRCUIT}

The MERCURY KEY.-This key is used for making and breaking a current by hand, and is constructed in various forms (see figs. 11 and 12).

In fig. 11 there are two cups, $\mathrm{C}^{1}, \mathrm{C}^{2}$, hollowed out in a vulcanite base and with two binding screws, $\mathrm{B}^{1}$ and $\mathrm{B}^{2}$, entering them from the
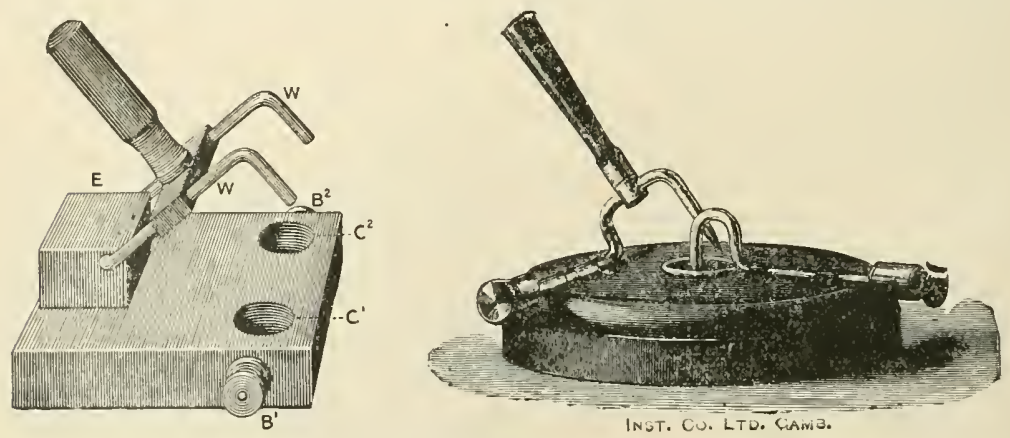

Figs. 11 and 12.-Two Foris of Mercory Key.

side. The cups are nearly filled with mercury, and can be connected by means of the stout bent copper wire $\mathrm{w}$ w which hinges through a

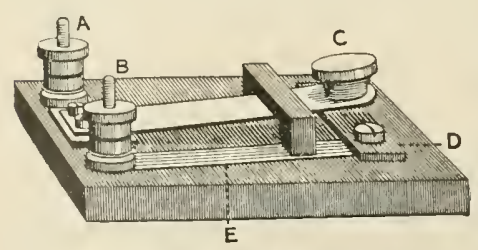

Fig. 13. - Sinple Forit of Spring Key. piece of vulcanite E. In fig. 12 there is a single mercury cup into which a wire dips to make contact with the binding screw.

The SPRING or CONTACT KEY (fig. 13) consists of a metal spring connected to a binding screw A. At its movable end there is a vulcanite knob c by which it can be depressed, and thus a platinum point on its lower surface brought into contact with a platinum plate 
on the brass plate $\mathrm{D}$, which is connected by the strip of copper $\mathrm{E}$ to the second binding screw $\mathrm{B}$. Whell interposed in the course of a circuit, the circuit will only be closed when $\mathrm{c}$ is depressed to lie in contact with $\mathrm{D}$.

DU BOIS-REYMOND'S FRICTION KEY (figs. 14 and 15) consists of two metal blocks $A$ and $B$ (fig. 14), each carrying two binding screws, fixed on an insulating base. The two blocks can be connected
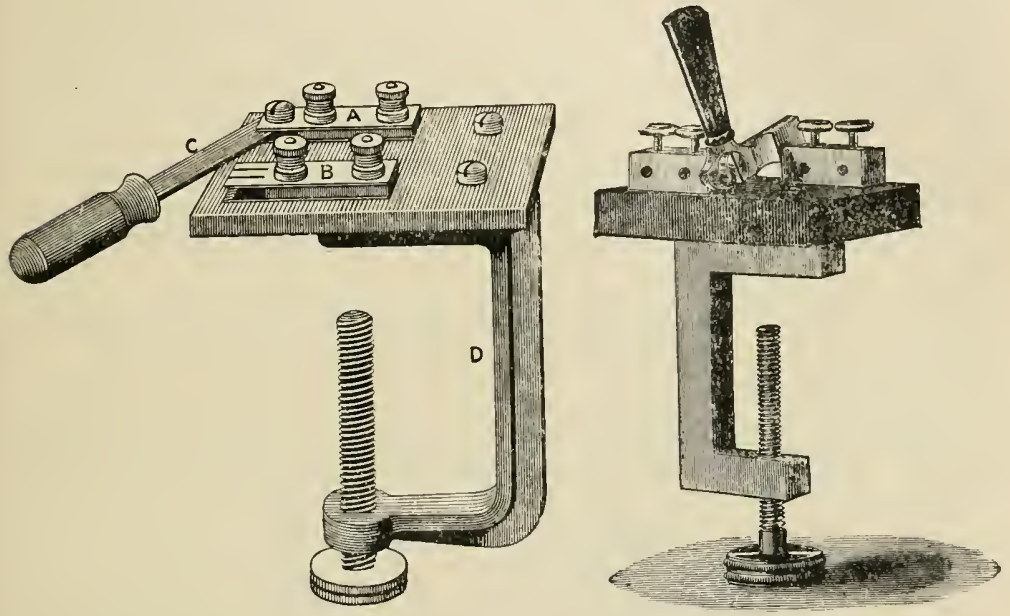

INST. CO. LTD. CAmB.

Firts. 14 and 15. - Two Foriss of the De Bois Kéy.

by a metal cross-bar c, which thus closes the key. This key is of very great service, and is employed in two ways indicated in the two accompanying figures (16 and 17), where it is represented as being used in the secondary circuit of an inductorium. In fig. 16 is shown the

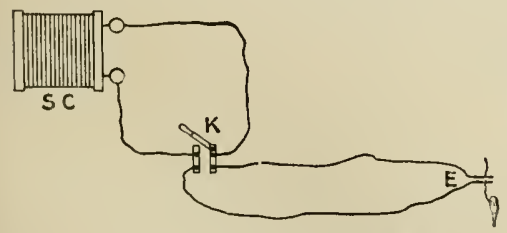

Fig. 16.-Plan of the Arraxgenent oh rile De Bois Key as a SHort-cirCUITLNG KeY.

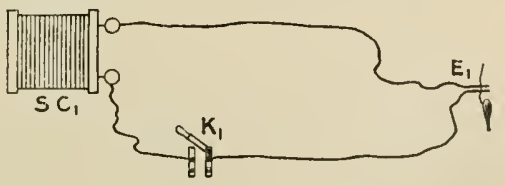

Fig. 17.-Arranged as a Simple Break KEY. NOT TO BE USED AFTER THIS Method in a Secondary Cilicuit.

arrangement in which it is used as a short-circuiting key. The two terminals of the secondary coil are connected by wires to two of the binding screws on the blocks, one to each block, and to the remaining two binding screws are connected the wires of a pair of electrodes, $\mathrm{s}$, 
lying under a nerve or other structure to be stimulated. When the key $k$ is open any current in the secondary coil can travel through the electrodes. If the key be closed, a current in the coil divides when it reaches the key, passing either through the key or to one electrode, thence through the nerve to the other electrode, and so back to the key. As the resistance of the key is very low compared to the high resistance of the piece of nerve, practically the whole of the current passes that way, or, in other words, the secondary coil is short-circuited. A Du Bois key is always to be used in this manner when in a secondary circuit. The second method of using the key is shown in fig. 17, where it is used as a simple key. The electrode wires $\mathrm{E}_{1}$ are represented connected to one terminal of the coil and to one block of the key $\mathrm{K}_{\mathrm{l}}$. The other terminal of the coil is connected to the second block of the key. When the key is closed any current in the coil can pass through the electrodes, but when the key is opened the secondary circuit is broken. The key can be used after this plan for making and breaking any battery circuit, but should not be thus employed in a secondary circuit.

POHL'S COMMUTATOR (fig. 18) consists of a wooden or vulcanite base in which are six mercury cups, to each of which a binding screw is connected. A rocker made of a vulcanite axis $\mathrm{H}$, to which two

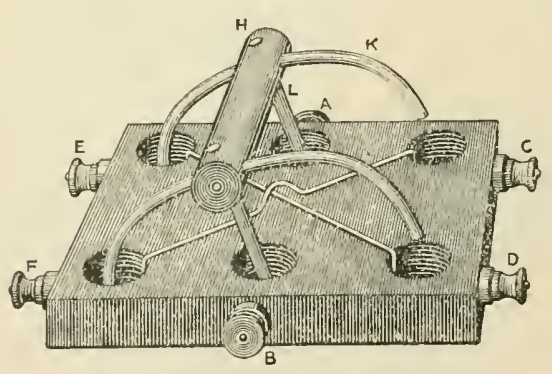

Fig. 18.-Pohl's Commetator. curved wires $\mathrm{k}$ and two vertical ones $\mathrm{L}$ are joined so that the vertical and curved wires of the same side are connected together, is so arranged that the two straight wires are supported in the cups A and $B$, and the curved wire may be made to dip into either pair of the four remaining cups. Two cross wires are also provided which connect $\mathrm{C}$ to $\mathrm{F}$ and $\mathrm{D}$ to $\mathrm{E}$. Supposing now that the positive pole of a battery is connected with A and the negative with $\mathrm{B}$, and the key is turned over so that the curved wires $\mathrm{K}$ dip into the cups $\mathrm{C}$ and $\mathrm{D}$, and if $\mathrm{c}$ and $D$ are connected by wires to any circuit, then the current enters at $A$, passes up $\mathrm{L}$ along $\mathrm{K}$ to $\mathrm{c}$, thence through the circuit to $\mathrm{D}$, and so to $\mathrm{B}$ and back to the battery. If now the rocker be turned over so as to rest in the cups $\mathrm{E}$ and $\mathrm{F}$, as shown in the figure, then the current enters at $A$, passes to $\mathrm{E}$, thence by one cross wire to $\mathrm{D}$, through the external circuit to $\mathrm{c}$, by the second cross wire to $\mathrm{F}$, and so back to $\mathrm{B}$. In the first position of the rocker the current in the external circuit 
was from $\mathrm{C}$ to $\mathrm{D}$, in the second position from $\mathrm{D}$ to $\mathrm{C}$, i.e. by moving the rocker the direction of the current in the external part of the circuit has been reversed.

This key can also be used in a second way by removing the crosswires, when two circuits can be closed by it, either from C to D or from E to F. Suppose, for instance, that the two ends of a muscle were connected by wires to $\mathrm{E}$ and $\mathrm{F}$, and the wires of a pair of electrodes upon which the nerve is lying to $\mathrm{C}$ and $\mathrm{D}$, then if the key be in the position of the figure a current entering at $\mathrm{A}$ and leaving at $\mathrm{B}$ is sent through the muscle, whilst if the rocker be rotated into the cups $\mathrm{C}$ and $\mathrm{D}$ the current through the muscle is broken, and instead is sent through the nerve. For the mode of connecting the key for such a purpose, see fig. 80 , p. 95 .

\section{A KEY FOR CUTTING OUT EITHER THE MAKE OR BREAK SHOCK}

This consists (fig. 19) of two spring lieys, one between $\mathrm{P}^{\mathrm{t}}$ and $\mathrm{p}^{2}$, closed when the spring $\mathrm{B}$ is brought into contact with the metal piece $\mathrm{D}$, and the other one between $s^{1}$ and $s^{2}$. The contact is made in each ease between two platinum
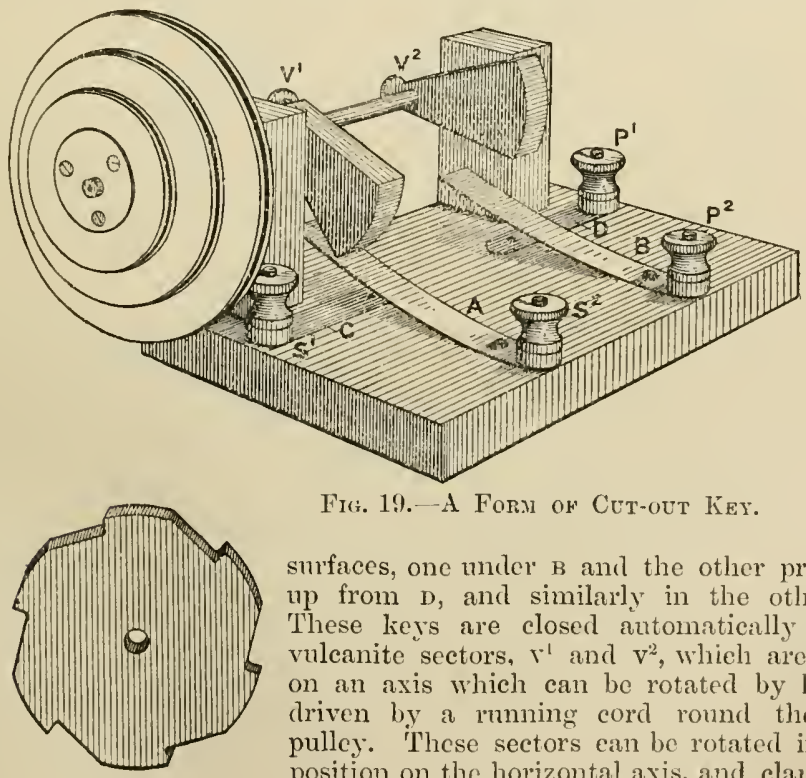

Fici. 19.-A Foris of Cet-out hey.

surfaces, one under $\mathrm{B}$ and the other projectiner up from D, and similarly in the otler liey. These kers are closed antomatically by two vulcanite sectors, $r^{-1}$ and $\mathrm{v}^{2}$, which are carricd on an axis which can be rotated by hand or driven by a rumning cord round the coned pulley. These sectors ean be rotated into any position on the horizontal axis, and clamped by screws. Fit up the liey $\mathrm{P}^{l} \mathrm{P}^{2}$ to malie and break a current through the primary, and $\mathrm{s}^{1} \mathrm{~s}^{2}$, so that it short-circuits the secondary when it is closed. Thus the two terminals of the secondary are connected, one to $\mathrm{s}^{1}$ and the other to $\mathrm{s}^{2}$, and the two electrode wires 
are commected to the same binding screws. If now the pulley be rotated in the dircetion of the hands of a wateh, and the sectors are in the position drawn in the figure, the sequence of erents is:-i. the spring $\mathrm{A}$ is brought into contact with $\mathrm{c}$, and therefore the secondary coil is short-circuited; ii. the spring $\mathrm{B}$ is brought into contact with $\mathrm{D}$, thus closing the primary; $\mathrm{a}$ make shoek is therefore induced in the secondary, which is, however, shortcircuiter because the spring $\mathrm{A}$ is still depressed; iii. the sector $\mathrm{v}^{1}$ glides off the spring $A$, which flies up, and the secondary coil is no longer shortcircuited; iv. the sector $\mathrm{v}^{2}$ leaves the spring $\mathrm{B}$, which flies up and breaks the primary cireuit, and the break shock now passes to the electrodes and through a nerve or muscle laid upon them. By fixing the sector $\mathrm{v}^{2}$ a little in advance of $\mathrm{v}^{1}$, only make shocks would be sent through the electrodes. When a more rapid series of stimuli is required, two notched wheels are provided to replace the sectors; these close and open the keys six times in each revolution, and one, as with the sectors, may be set a little in advance of the other, and so either make or break shocks sent through the electrodes as desired.

Fit up the key as directed, and placing the electrodes upon the tongue, rotate the key, and show that the one or other shock can be cut out as required.

Experiment 1.- Show that the break shock is greater than the make shock in the following way. Comnect the primary coil with a battery and mercury or' spring key as in fig. 20. To the secondary coil attach a pair of wires, and remove the coil to some distance from the primary. Hold the two
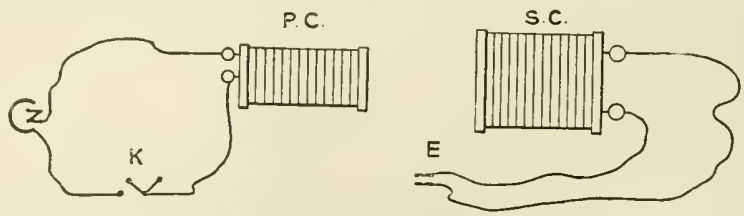

Fig. 20.-Arrangement of Apparatus for Making Use of Single Ixduced Shocks.

iree ends of the wires on the tip of the tongne, and make and break the primary circuit by opening and closing the mercury key. At first nothing is felt. Now gradually move up the secondary coil, testing each new position by opening and closing the key in the primary circuit. At last a position will be found at which a shock is perceived at break and none at make. Make a note of the position of the secondary coil with respect to the fixed scale. Move up the secondary still further, noting that the break shock becomes progressively stronger, and at last a position is reached at which a shock is felt on making the current. This position is to be noted and contrasted with that previously observed for

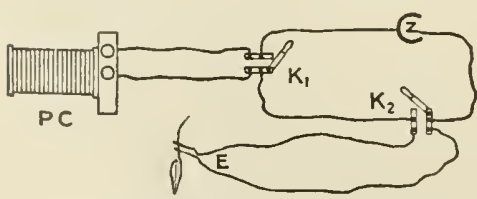

Fig. 21. -Arrangement of Apparatus to

Show the Break Extra-current. the break shock. The experiment also clearly shows how convenient the coil is for modifying the strength of stimulus to any required degree.

Experiment 2. - To demonstrate the break extra-current arrange the apparatus as in fig. 21, applying the electroiles $\mathrm{E}$ to the tongne. First close the key $\mathrm{k}_{1}$; on now closing the kry $\mathrm{k}_{2}$ the current is short-circuited, and none passes through the tongue; on opening $\mathrm{K}_{2}$ all the current passes through 
the tongue. Neither on opening nor closing the key $\mathrm{K}_{2}$ is any distinet shock felt unless the battery is very strong. Now open the key $\mathrm{K}_{1}$ and again open and close $\kappa_{, y}$. Each time the key $\mathrm{r}_{\mathrm{z}}$ is opened the current is sent through the tongue, and the resistance being very high there is a sudden fall in strength of the curvent. On opening a distinct shock is felt. This is due to the extra-current brought about by the sudden fall in strength, inducing currents in the turns of wire of the primary coil $\mathrm{P}$ c. 
PITH A FROG.-Pass your nail along the back of a frog's skull until the groove between the skull and the first vertebra is felt, and then insert the point of a fine scalpel between these two, and so divide the central nervous system transversely at about the level of the medulla. Now insert a blunt-pointed seeker into this aperture and pass it forward into the skull cavity, so as to destroy the brain, and then downwards into the vertebral canal, and thus destroy the spinal cord.

MAKE A NERVE MUSCLE PREPARATION,--The simplest and one of the most convenient muscles to isolate for experiments is the gastrocnemius. Its anatomical relations are shown in figs. 22 and 23. To prepare it together with its nerve, pith a frog, and cutting through the spinal column one vertebra above the sacrum, remove all the soft parts in front down to the pubis, including the viscera, taking care not to injure the branches of the sciatic plexus lying on the posterior wall of the abdominal cavity. If the sacrum be now firmly held, the skin over the back of the iliac bones can be drawn down, and the whole of it drawn off the two legs, thus laying bare the muscles of the thigh and leg. The tendo Achillis is cut across below the ankle-joint, and with its sesamoid bone dissected free up to the belly of the gastrocnemius, which is then isolated from the tibia and fibula right up to its insertion into the femur. The head of the tibia is then cut through just below the knee-joint. Next proceed to isolate the sciatic, which will be found lying between the biceps, B, fig. 23, and semimembranosus, sm, on the posterior surface of the thigh. Carefully separate these muscles, and follow up the nerve to the pelvis, cutting through its branches as they are laid bare. The nerve should not be touched with metal instruments, and in its separation should not be allowed to be covered with blood from the vessels which accompany it. Next cut through the muscles attached to the urostyle, and divide 
the vertebræ in the mid line into two symmetrical halves. Lift up the muscles which have been cut from the urostyle, and turn them outwards, so as to expose the sciatic, which can then be completely isolated up to its three constituent cords, and so to the vertebræ. Cut through the joint between the vertebræ and the ilium, and the vertebræ can then be picked up, and by this means the nerve lifted and its isolation completed down to the lower end of the femur, where it divides into two branches. It is then laid on the gastrocnemius while the muscles are separated from the femur, the triceps from the outer side, and the adductors from the inner. The femur is then cut through at

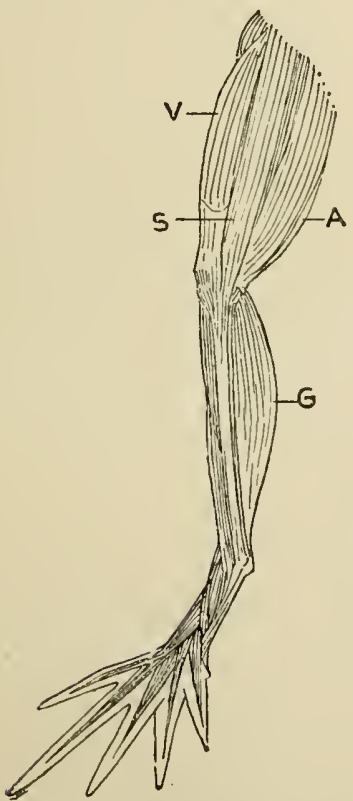

Fig. 22.-Leg Muscles of the Frog SEEN Frou the INNER Side. A, Gracilis. s, Sartorjus. v, Vastus Internes. G, Gastrocnemios.

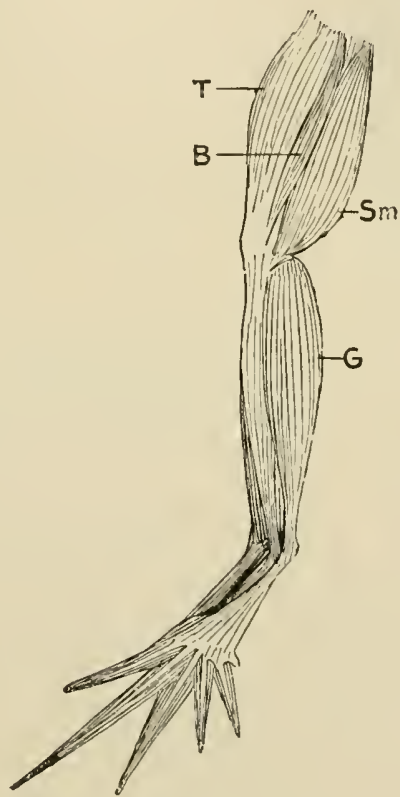

Fig. 23. - Leg Muscles of the Fror SEEN Fros the OUter Side. T, Triceps. B, Biceps. sm, Semimembranosus. G, Gastrocnemitus.

about its upper third and the preparation is complete. Fig. 24 is a drawing of such a nerve-muscle preparation, where $F$ is the femur and $\mathrm{K}$ the knee-joint ; $\mathrm{G}$ is the gastrocnemius and $\mathrm{T}$ the tendo Achillis with its sesamoid bone $\mathrm{s}$. The nerve $\mathrm{N}$ stili remains attached to a piece of the vertebral column $\mathrm{v}$, which serves as a convenient means of handling the nerve. At $\mathrm{N}_{1}$ is the branch of the nerve to the gastrocnemius. The femur can be clamped in the muscle forceps, and thus a rigid support is given to its upper end. A fine thread is tied round 
the tendon, or this is pierced by a bent pin, and thus the lower end attach $3 d$ to the lever of a myograph (see fig 31, p. 25).

If a crank lever is to be used it is not necessary to thoroughly isolate the femur, but its lower end can be directly fixed to the cork plate of the myograph by a needle which is passed through the bone.

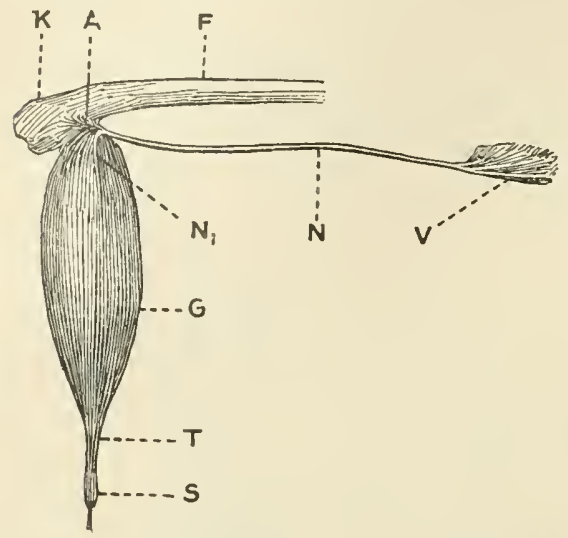

Fig. 24.-Gastrocnemings-sciatic Preparation. In many cases, too, it is not necessary to completely isolate the nerve up to the vertebræ.

DIRECT AND INDIRECT EXCITATION OF MUSCLE. A muscle may be made to contract by a stimulus applied to the muscle mass itself, when the excitation is termed direct, or it may be caused to contract by a stimulus applied to its nerve, which stimulus then travels down to the muscle. This is indirect excitation. Test this by applying the electrodes first to the nerve and then to the muscle, and sending an induced current through the electrodes.

\section{THE MOIST CHAMBER}

In all instances in which we are experimenting upon an excised muscle and nerve, it is of the greatest importance that they should be protected from drying. To secure this it is necessary either to immerse them in some fluid which exerts no harmful effect upon them, such as defibrinated ox-blood, or to place them in an enclosed airchamber in which the air is kept moist. This latter is termed a moist chamber, and is of different form according to the myograph employed. It consists of a glass cover to the myograph, in which is an aperture through which a thread may pass to connect the muscle to the recording lever. The air in the chamber is kept moist by placing in it a few pieces of blotting-paper wetted with normal saline solution.

Experiment 1.-Utilise this nerve-muscle preparation to prove that the break shock is stronger than the make shock. Arrange the apparatus in the same way as in Experiment 1, p. 14 (see fig. 20), placing the nerve upon the pair of electrodes. Gradually decrease the distance between the two coils as in that experiment, and make notes of the positions of the secondary coil when a twitch occurs-(1) at break of the primary circuit, (2) at make.

Experiment 2.-By using the arrangement previously described and shown in fig. 8, p. 7, show that, by the introduction of a deriving circuit of 
low resistance in parallel with the primary coil, the induced shocks are rendered of nearly equal value. Test this on the nerve-muscle preparation. On varying the position of the secondary coil as in the preceding experiment, it will now be found that the strength of the break shock has become nearly equal to that of the make shock, which has also been somewhat reduced.

Thus in one experiment it was found that the farthest position of the secondary coil from the primary at which a break shock caused a twitch of the muscle was $26 \frac{1}{2} \mathrm{~cm}$. A make shock was first effective when the coil was brought up to $10 \frac{1}{2} \mathrm{~cm}$. With the deriving circuit of low resistance as in experiment 2 the break shocks first produced a twitch when the coil stood at $10 \mathrm{~cm}$, and the make shock was effective when the coil stood at $9 \frac{1}{2} \mathrm{~cm}$.

Experiment 3.-Demonstrate upon the nerve-muscle preparation the existence of the break extra-current, arranging the apparatus as in Experiment '2, p. 14 (fig. 21).

Experiment 4.-Denonstrate the make extra-current, arranging the apparatus as in fig. 25. A current is sent through the primary coil and electrodes arranged in parallel and with a $\mathrm{Du}$ Bois key $\mathrm{K}^{2}$ interposed so that both may be short-circuited. Interpose a friction key $\mathrm{K}^{1}$ and a resistance-box $\mathrm{R}$ in the main circuit. Also place a key $\mathrm{K}^{3}$ in the electrode circuit. The current on reaching the key $\mathrm{K}^{2}$ divides, and as the resistance of the piece of nerve across the electrodes is very high, most passes through the primary coil, which therefore acts as a deriving circuit. Close the key $\mathbf{K}^{1}$ and open $\mathrm{K}^{2}$, and now interpose enough resistance at $R$ until opening and closing $k^{3}$

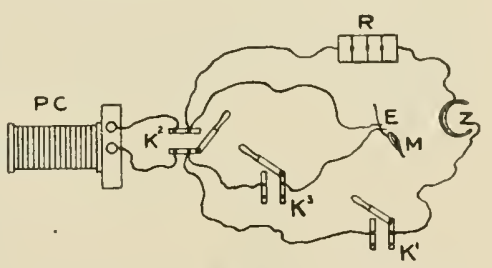

Fig. 25.-Arrangement of Apparatus for Showing Malie Extra-current. gives no contraction of the muscle. Next, with $\mathrm{K}^{-1}$ and $\mathrm{k}^{3}$ closed, open and close $\mathrm{r}^{2}$. Each time $\mathrm{K}^{2}$ is opened the muscle contracts, stimulated by the make extra-current in the primary coil, for the strength of constant current at the same time sent through the nerve has, by increasing the resistance $\mathrm{R}$, been reduced until it no longer was able to stimulate on make. On closing the key $\mathrm{K}^{2}$ the currents through both primary and nerve are short-circuited, a break extra-current is produced in the primary, which, however, is short. circuited by the key $\mathrm{K}^{2}$. The break of the current through the nerve is not sufficient to stimulate, and the muscle does not contract.

If the key $\mathrm{K}^{2}$ be kept open and $\mathrm{K}^{1}$ closed and opened, a contraction occurs both at make and break. This arrangement thus demonstrates both make and break extra-currents.

\section{MAKE A GRACILIS AND SEMIMEMBRANOSUS PREPARATION}

First study the relations of these muscles as given in figs. 22 and 23. Pith a frog and dissect away the skin from the thigh, carefully cutting through the fibres of the rectus internus minor, which are inserted into the skin on the adductor surface of the thigh. The muscles can then be readily made out on the inner side of the thigh separated from one another by the rectus internus minor. The gracilis, or rectus internus major, $\Lambda$, is to be scen from the front of the thigh, being in relation on its outer edge with the 
ulductor brevis, adductor magnus and sartorius, s. The semimembranosus, $\mathrm{s} m$, is seen on the posterior surface with its outer border in relation with the pyriformis above and the biceps, $\mathrm{B}$, below. Both muscles arise above from the symphysis pubis, and below are inserted by tendinous aponeuroses into the tibio-fibula. Cut through the aponeurosis at the outer border of each muscle, and then separate each from the subjacent muscles, viz. the adductors and semitendinosus. Isolate the muscles right down to their lower insertion and cut through the tibio-fibula just below this, and then divide the femur a little above the knee-joint. By holding the piece of bone thms isolated the two muscles can now be easily separated right up to the symphysis. The semitendinosus nsually tends to separate with them, and may be remored later by cutting through its lower attachment, then dissecting it away from the gracilis, or finally dividing its two heads of attachment to the pelvis. The other muscles attached to the symphysis are now ent through, and the head of the femur disarticulated from the acetabulum. In many cases it is convenient to make a second preparation in a similar manner from the opposite leg; but if this be not reyuired, the whole leg may be removed, disarticulating at the acetabulum. The great adrantage of this preparation is that we have a mass of muscle in which the fibres are very nearly straight, and are of a good length. With a double preparation the muscles can hang side by side, and so the tranverse section is doubled. The upper end can be conveniently fixed by passing a strong needle through the acctabula. With the two preparations dissected out they can also be hung one below the other, being united by a piece of the symphysis, and thus a muscle of donble length is obtained.

MAKE A HYOGLOSSUS PREPARATION.-One of the simplest

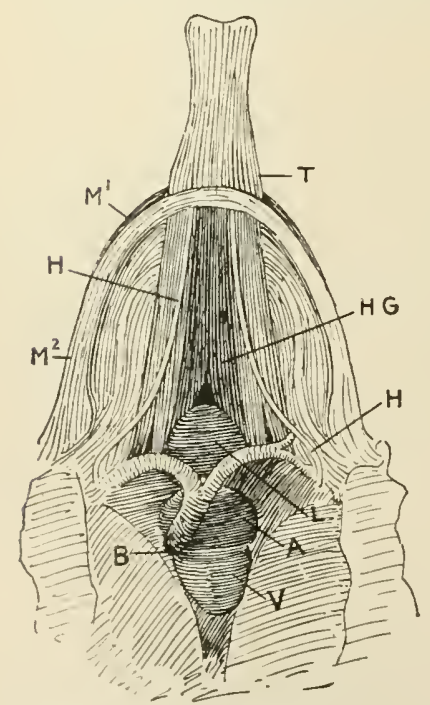

Fig. 26.-The Relations of the Hyoglossus, h G, in the Frog. and most convenient muscle preparations that can be obtained from a frog is the hyoglossus muscle. Fig. 26 shows the general course and arrangement of the muscle. It is attached to the anterior edge of the body of the hyoid cartilage, and from this the fibres run forwards to meet in the mid.line with the muscle of the opposite side. The two then run forward as two bands to the apex of the lower jaw, and thence into the substance of the tongue. In the tongue the fibres run towards the tip and the muscle gradually ends by becoming inserted into the submucous connective tissue of that organ. It is supplied by the hypoglossal nerve (H, fig. 26). To utilise the muscle when we wish to stimulate directly, all that is necessary is to lift up the lower jaw and cut through the joint between the two jaws on either side, extending the in- 
cisions down to the shoulder girdle. The lower jaw is then pulled slightly forwards, and by a single transverse incision at the upper edge of the shoulder girdle the whole of it is removed. It is now placed mucous surface upwards, the tip of the tongue lifted up and either transfixed with a hook, or a fine thread is tied round it. The tongue is then turned forwards and extended out of the mouth. The body of the hyoid cartilage now stands out clearly, and this may be transfixed by a pin, and in that way fixed to the cork of a myograph, or the cartilage may be directly clamped in a muscle forceps. The thread or hook may then be attached to the writing lever. The great advantage of the preparation is that the muscles are composed of long fibres strictly parallel to one another, which are completely protected from any injury during the preparation, because the muscle itself is not exposed. Remaining in situ the whole time, they are protected from drying by the mucous membrane of the tongue and mouth, and on the ventral side by the slsin of the jaw.

If we wish to stimulate indirectly, the two hypoglossal nerves can be easily isolated and laid upon electrodes. The only disadvantage lies in the small size of the muscle, but the many advantages which it possesses give, in the greater number of experiments, full compensation for that.disadvantage.

\section{THE GRAPHIC METHOD}

Iost of the movements carried out by the different parts of the body, and which it is our object to study, are performed at so rapid a rate that the unaided eye is only able to give us a judgment of the broad outlines of the movement. By it alone we are quite unable to gain any accurate knowledge of the details of a particular movement. For instance, if we expose the heart of a recently killed frog, and watch it beating, it is difficult to be certain that the auricular beat precedes the ventricular, and in many cases it is quite impossible to determine with any certainty whether the contraction be carried ont at a faster or slower rate than the dilatation, or, if there be a difference, to determine the amount of that difference. Still more is the difficulty perceived if we turn our attention to a more rapid movement, such as a single twitch of a frog's muscle, where the whole cycle of movement is so rapid that we are quite unable to accurately judge of its amount, or of any variation in the rate of its contraction or relaxation. We require, then, some means of obtaining a permanent record of each movement which we may afterwards study at our leisure; and this means is afforded us by what is termed the graphic method, the general principle of which is that the part in movement is marle to record its movement by writing it upon a surface. Thus if we wish 
to record the amount of contraction of a frog's muscle we may fix one end to some rigid support and to the free end attach some form of writing-point, which is made to record its movement upon a piece of paper so placed that the point, during its movement, is always in contact with the paper. Where the amount of movement to be recorded is small, it is readily magnified by some form of lever such as one of those represented in figs. 31 and 37 . We in this way obtain a straight or eurred line which gives us at once a permanent record of the amount of movement performed, or of some multiple of it. We still have one important point to determine in the consideration of any movement, viz. the time occupied. Thomas Young was the first to show how we might obtain measurements of time with very considerable accuracy. He pointed out that if a surface be moved in a given direction at a constant rate, lines measured parallel to the direction of motion indicated time, and that to determine the value of those lines all that was necessary was to fix a very light style or marker to a vibrating rod, held so that the style was in contact with the moving surface and its movements at right angles to the direction of motion of the surface. If the time of oscillation of the rod be known, the rate of movement of the surface is directly determined. This time measurement was perfected by Duhamel by employing a tuning-fork to one prong of which a light writing-point is fixed. The rate of ribration of the tuning-fork can be determined with rery great accuracy, and hence the rate of morement of the surface can be determined with the same accuracy. The recording surface, which is most convenient and which is usually employed, consists of a smooth and highly glazed surface of paper, which is covered with a thin deposit of carbon, obtained by holding it in a smoky flame of burning gas, camphor, turpentine, or some other substance. The writing-point is made of metal, glass, or moderately stiff paper eut to a sharp point, which is

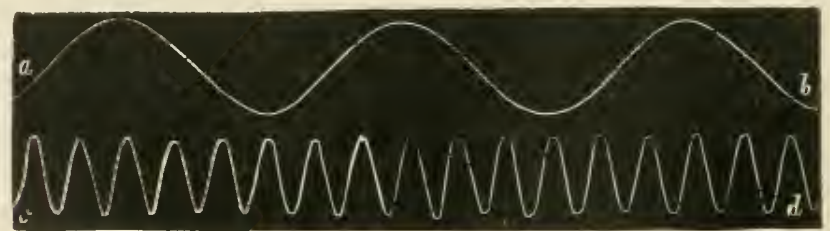

Fig. 27.-Two Records of the Vibrations of a Tuning-fork Vibrativg at the Rate of 10 per sec. The Tracing $a b$ was taken while the Recording Surface was Moring hore Rapidix thax during the Record c d.

then made to scratch the smoked surface, and so remove some of the black deposit and bring the white surface of the paper into view. The 
shape of surface which is most generally useful is that of a cylinder which can be set rotating about its long axis by clockwork or some other means. In fig. 27 are reproduced two tracings taken by a tuningfork, which vibrated at the rate of ten per second, the cylinder being made to rotate at two different rates. The distance between the summit of one curve and that of the next curve represents the space travelled over by the surface in $\frac{1}{10}$ second. This distance in the tracing $a b$ is $2.85 \mathrm{~cm}$, or in one second the surface travelled $28.5 \mathrm{~cm}$. In the lower tracing the rate is found to be $4.8 \mathrm{~cm}$. per second, if we measure the distance between one summit and the tenth following.

The recording of time by means of a tuning-fork possesses the disadvantage that the vibrations soon cease, especially if the rate of vibration be rapid. To obviate this, a method commonly employed is to record by means of a chronograph (fig. 28), actuated by an electrical current made and broken at some definite known rate by a special piece of apparatus. The chronograph

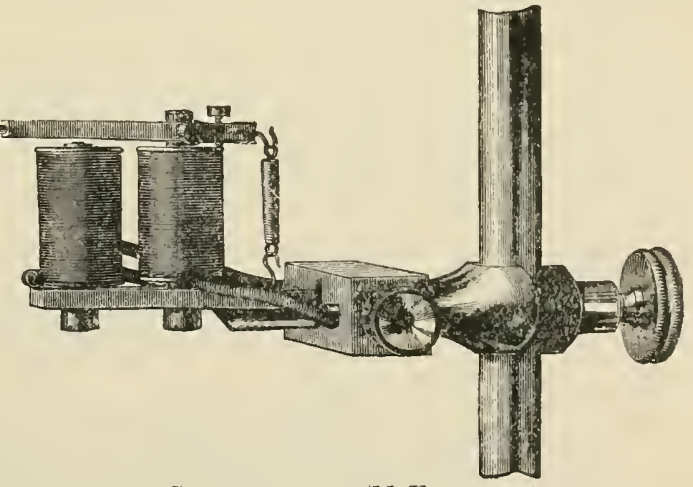

Fig. 28. - A Thue-mariker or Cimonograpil. (McKendick.)

(fig. 28) consists of a small electromagnet and a movable armature, to which is attached a writing-point. Each time the current is closed the armature is attracted and the writing-point moves downwards. The rate of vibration of the writing-point thus depends upon the rate of make and break of the current employed. The current may be antomatically closed in a regular manner in several ways. Where the rate required is slow a pendulum clock is very frequently used; when a more rapid rate is required, a tuning-fork, to one prong of which a platinum wire is attached, so that with each vibration the wire completes a circuit either by touching a platinum surface or by dipping into mercury. The tuning-fork is kept vibrating indefinitely by means of an electromagnet.

A very convenient time-marker is shown in fig. 29. It consists of a stiff steel band s firmly clamped at one end by the metal cross-bar c. Attached to it is a heavy weight $\mathrm{w}$, by altering the position of which we are able to modify, to a certain extent, the rate of oscillation. The oscillation of the spring is communicated to the vertical bar $\mathrm{E}$, and thus to a lever $\mathrm{B} \mathrm{E}$, to which a writing lever $\mathrm{L}$ is attached. The writing lever $\mathrm{L}$ makes an angle with $\mathrm{B} \mathbf{E}, \mathrm{so}$ 
that the spring s does not touch the writing surface. With w in the position drawn, the oscillations are at the rate of two per second. When $w$ is moved

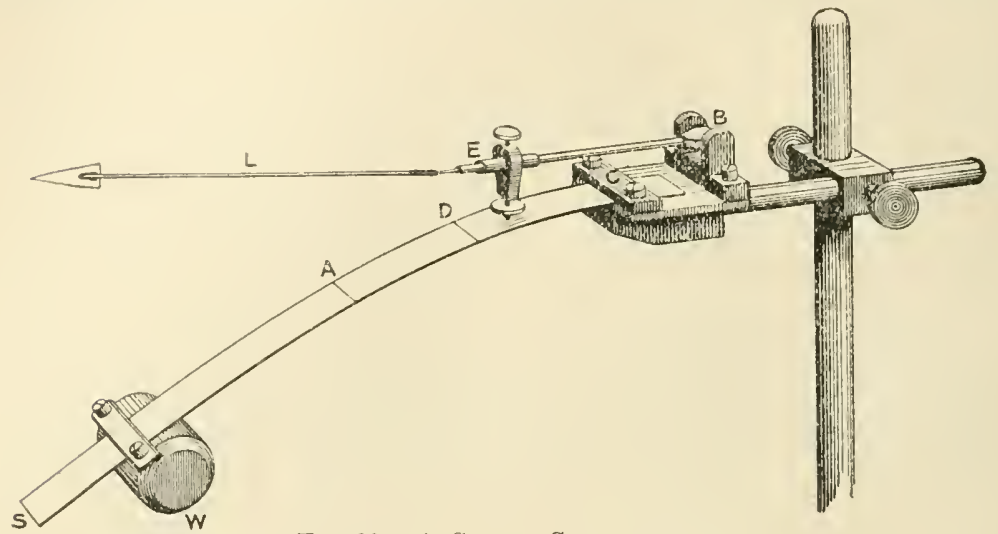

Fig. 29.-A Sprixg Chronograph.

to the transverse mark $A$ the rate becomes four per second, and when at $D$ eight per second. As the weight is heavy, when once the spring is started vibrating it keeps on for a snfficient length of time for most experiments.

In a recording cylinder such great differences of speed are required for

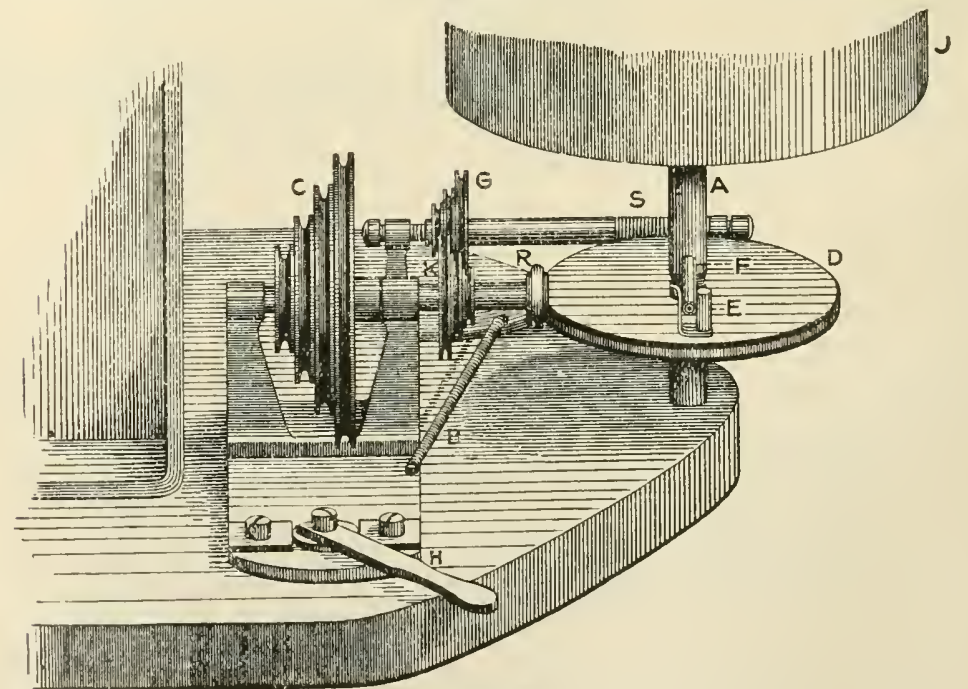

Fig. 30.-Lower Part of Drun to show Method of Driving the Cylindei at DiffEReNT Rates.

different purposes that it is often difficult on the same drum to obtain sufficient variations. For rapid rates of movement a common plan is to drive a 
friction wheel, by means of clockwork or a running cord, which can be thrown into contact with a second wheel on the axis of the drum (see fig. 37). For slow rates the most satisfactory method is to rotate the drum by means of a tangent screw. The drum represented in fig. 30 combines these two, so that one or the other plan can be used by simply changing the position of a lever $\mathrm{H}$. The figure represents the base of the drum only, the cylinder and upper fittings being the same as those seen in fig. 37. The axis of the drum rests on the steel point of a short vertical rod round which a brass disc $D$ rotates in a collar. On the dise is a little upright $\mathbf{E}$ which is placed in contact with a bar $\mathrm{F}$ serewed into the drum-spindle $\mathrm{A}$. $\mathrm{F}$ and $\mathrm{E}$ are kept in contact by a brass spring, so that the drum is rotated by the lrass dise. The coned pulley $\mathrm{c}$ is driven by a running cord, and on the same axis is a smaller coned pulley $\mathrm{K}$ and a brass ring $\mathrm{R}$ covered with rubber. When $\mathrm{R}$ is brought into contact with the edge of the disc $\mathrm{D}$ the latter is set in movement, and thus gives a rapid movement to the drun. The coned pulley $\mathrm{x}$ by an endless cord drives a second pulley $\mathrm{g}$ on a second axis, the end of which is a screw. The screw fits into a toothed projection on the rim of the wheel $\mathrm{D}$, so that when $\mathrm{s}$ and $\mathrm{D}$ are in contact, as in the figure, the rotation of $\mathrm{s}$ gives a slow movement to the disc $D$. These two axes are fixed on a base piroting about a point hidden in the drawing by pulley c. When the handle $\mathrm{u}$ is carried over to the left, the rubber dise $\mathrm{r}$ comes into contact with the dise $\mathrm{D}$, and the serew $\mathrm{s}$ is removed. When, on the other hand, the handle $\mathrm{H}$ is to the right, $\mathrm{R}$ is removed and s bronght into contact with the disc.

As a general rule, the various movements we have to record are small in extent, and it therefore becomes necessary to magnify them at the time we record thent. This is usually effected by employing some form of lever, the extremity of which is made of a writing-point,

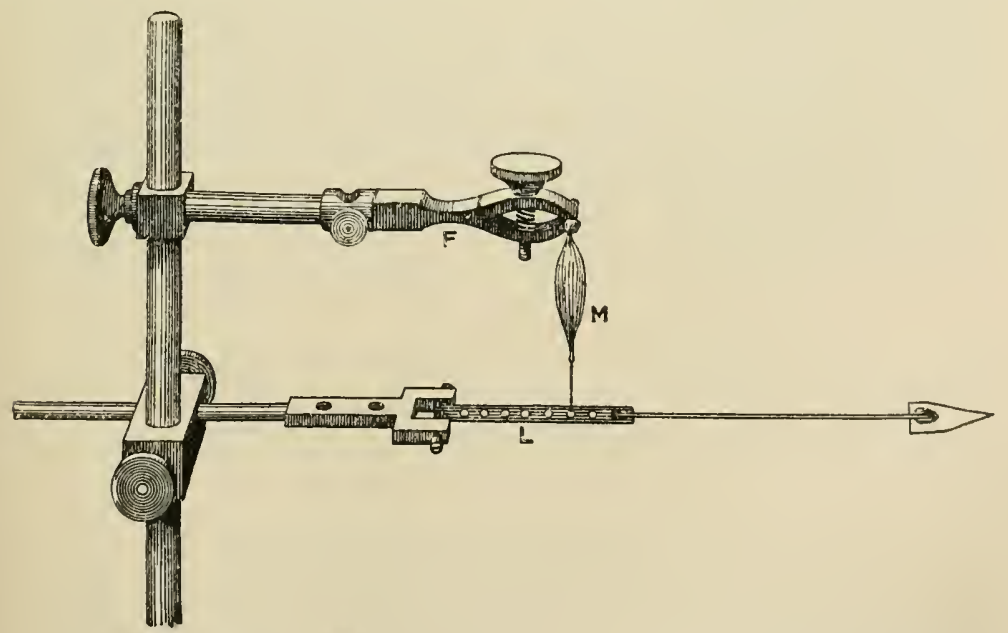

Fig. 31.-Musche held in Muscle-forceps F and attached to Simple Lever L.

and which is fixed, at a point near its axis, to the muscle or other tissue whose movements are to be recorded. The degree of magnifica- 
tion then varies inversely as the distance of the point of attachment from the axis. Fig. 31 represents such a SIMPLE LEVER L, which is represented as arranged for recording the movement of a muscle $\mathrm{I}$, whose upper end is held firmly in the MUSCLE FORCEPS F. All recording levers should be made as light as possible, consistent with sufficient rigidity to prevent distortion of the record by vibrations set up in the lever itself. The question of lightuess is of the greatest importance when rapid movements are to be recorded.

Another form of lever which is also very commonly employed is the CRANK LEVER. This consists of a lever with two arms fixed at right angles to each other. It is represented in fig. 37 , p. 31, as being used for recording a simple twitch of a muscle. One of the two arms is long, and when used is fixed horizontally. This is the writing lever. The other is fixed vertically, is much shorter, and is the lever to which the muscle is attached. The muscle in this instance lies horizontally, so that with a crank lever the movement of the writing point is at right angles to the direction of the movement recorded.

When we wish to excite a muscle electrically it is necessary to have a pair of electrodes by which the shock may be carried to the muscle or nerve to be excited. There are very many forms of these which can be employed.

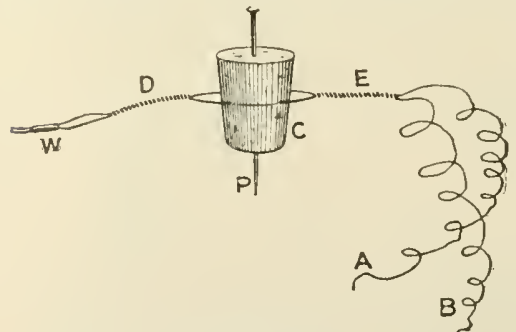

Fig. 32.-Simple Foris of Wire Eliectrodes. A simple form, readily made, is shown in fig. 32 . Two very thin and flexible copper wires, $A$ and $B$, covered with silk are taken and twisted together at $\mathrm{D}$ and $\mathbf{E}$. A small cork $\mathrm{C}$ is taken transfixed by a pin $P$ and two shallow cuts made in it. The wires are then forced into the cuts, as seen in the figure. This holds the wires firmly. Near the ends of the wires a drop of melted sealing-wax $w$ is fixed, so as to hold the wires parallel to one another and about $1 \mathrm{~mm}$. apart.

The wires are then cut off about $4 \mathrm{~mm}$. beyond the wax, and these projecting pieces bared by scraping off the silk insulation. In many cases it is a further advantage to imbed the uncovered points in wax, and only expose the wires for about $1 \mathrm{~mm}$., and on one surface only. This tends to prevent escape of the current to sturrounding parts.

\section{MINIMAL AND MAXIMAL EXCITATION}

If the strength of the excitation be varied, it is found that the response of the muscle varies in amount. This should be studied in the following way.

Experiment 5.-Cover and blacken a drum. Dissect out a muscle and attach it either to a simple-lever or to a crank-lever myograph. Fit up the 
exciting apparatus with a contact key in the primary and a Du Bois key in the secondary. The muscle may be stimulated either directly or indirectly. Arrange the electrodes accordingly. Remove the secondary coil to some distance from the primary. Bring the writing-point to the drum surface, and while the latter is at rest close and open the key in the primary. No contraction results either on make or on break. If one occur move the secondary further from the primary. Gradually move the secondary up to the primary, when a position will be found at which a slight twitch will occur at break. This is recorded as a vertical line on the drum. Now turn the drum by hand through about $.5 \mathrm{~cm}$. Move up the secondary coil $1 \mathrm{~cm}$. and stimulate as before. Repeat gradually, increasing the strength of the stimulus and moving the drum after each contraction has been recorded. At a certain position the make shock will be found to canse a contraction as well as the break. After a time it will be found that a further increase of the strength of the stimulus does not lead to an increase in the height of the contraction.

Fig. 33 records an experiment carried out in this way. It was obtained from a gastrocnemius with indirect stimulation, and a magnification of 5 . The first indication of a contraction was on break with the secondary coil at $17 \mathrm{~cm}$. of the scale. This strength of stimulus is called the MINIMAL STIMULUS, and the contraction is

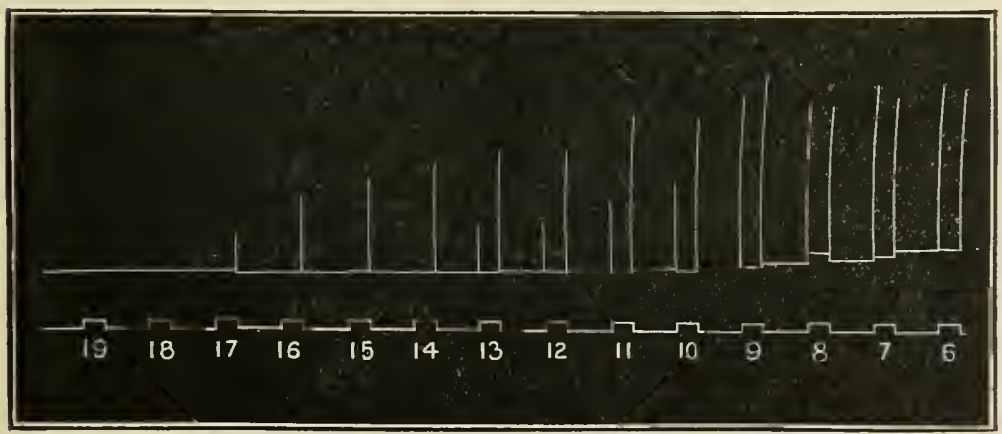

Fig. 33. - Heights of Contraction of a Muscle with Differext Strexgths of Stimoli. The Numbers Refer to the Distances of the Secoxdary Coil. The Interropted Line above Shows tire Instants at which the Primary Culrent was Made and Broken. A Rise in this Line indicates Make, a Fall Break.

also termed minimal; any strength of stimulus lower than that was for this muscle sub-minimal. As the stimulus was increased it is seen that the contractions on break increased at first rapidly, and then more slowly, but that beyond $9 \mathrm{~cm}$. the height did not increase. The stimulus at $9 \mathrm{~cm}$. was therefore a MAXIMAL STIMULUS. All strengths of stimulus below this were SUB-MAXIMAL. A contraction on make was first obtained when the secondary coil stood at $13 \mathrm{~cm}$., and this rapidly increased in amount till it reached a maximum at $9 \mathrm{~cm}$. 
The tracing also shows one other point of some importance. It is to be noticed that the heights of the break contractions do not show a perfectly uniform gradation, but offer some irregularities. This is due mainly to irregularities in the strength of the stimulus, for the induced shock at break is in reality compound, and caused mainly by the break of the current, and also by the break extra-current which sparks across in quite an irregular manner at the instant the break is effected.

\section{UNIPOLAR EXCITATION}

Experiment 6.-Set up the coil to give single shocks, and at first only attach one wire to the secondary coil. Excise a nerve muscle preparation, and placing it upon a dry glass plate put the single wire from the secondary coil under the nerve. On opening or closing no contraction occurs. Next insert a second wire in the remaining terminal of the secondary coil and attach its other end to a gas pipe and so to the earth. A contraction will now oceur both on opening or closing the primary circuit.

Thus it is seen that, in the latter case, the amount of current which passes through the earth and the glass plate is sufficient to stimulate the nerve. It is in order to avoid excitation in this way that the Du Bois key is used as a short-circuiting key in the secondary circuit.

\section{RECORDING MOVEMENTS BY MEANS OF TAMBOURS}

In recording movements of different parts of the body, it is often necessary to be able to transmit that movement to some little distance because the part cannot be conveniently brought sufficiently near to

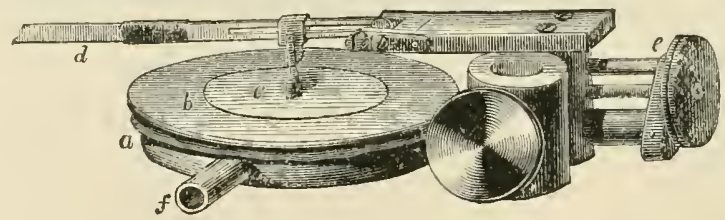

Fig. 34.-Marey's Form of Recorbing Tambour.

the recording surface to be able to write its movements directly upon the surface. When this is the case, one of the most convenient methods is to employ a pair of tambours, one of which is termed the receiving tambour and the other the recording tambour.

Each tambour consists of a shallow circtlar metal box whose upper surface consists of a rubber membrane so that it is air-tight. A tube leads 
into the interior of each, and the two tambours are through these connected by a piece of rubber tubing. Then thus comnected a pushing-in of the nembrane of the receiving tambour causes a corresponding rise of the membrane of the recording tambour, and to the same extent if the two tambours are of the same size. The form of the receiving tambour varies according to the purpose for which it is intended. The form of the recording tambour is shown in fig. 34 . The flat metal box, provided with a side tubular $f$, is represented at $a$. This is corered above by the rubber membrane $b$, to the centre of which is attached a metal dise $c$ with a vertical jointed rod which moves the recording lever $d$. The anount of magnification may be varied by altering the position of the jointed rod with respect to the axis of the writing lever.

Another form of tambour is represented in fig. 35. It consists of an oblong vulcanite base on whose upper surface is a shallow circular carity into which the metal tube $\mathrm{T}$ opens. This is covered with thin ruber membrane, which is attached to the vulcanite with a little Canada balsam. The upper surface of the rubber is covered by a brass plate $P$ with a central circular aperture through which the mbber $R$ is seen. The movements of the men. brane are transmitted by the corli $c$ to the writing lever $L$. The axis of this lever is held on a rod, which can be clamped in any position by the screw $\mathrm{R}$, and adjusted to any height by a vertical rod passing through the vulcanite base and fixed by the serew $\mathrm{s}^{\mathrm{I}}$. The advantages of this form are that the

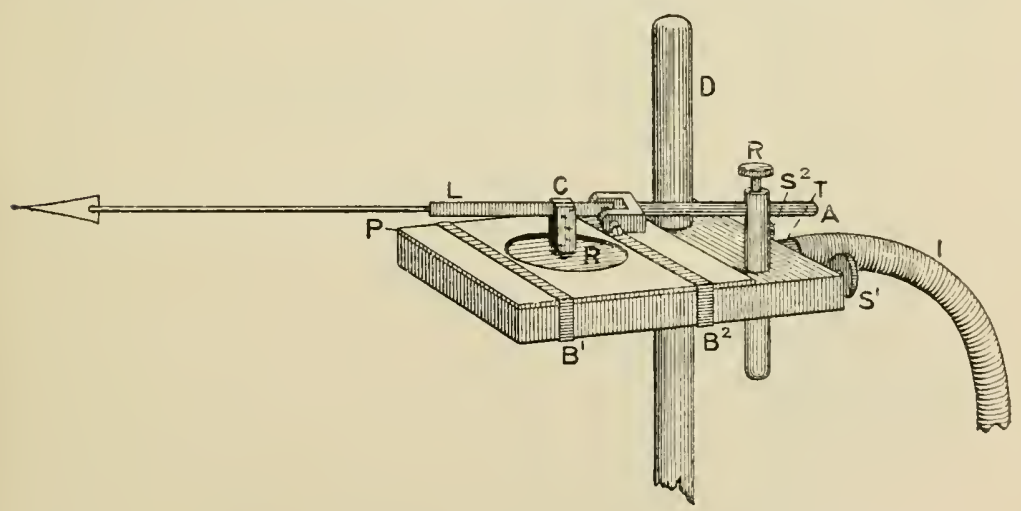

Fig. 35.-A Seconi, Fona or Recoming Tambodr.

rubber membrane is very quickly replaced, and is easily made air-tight. The netal plate $\mathrm{P}$ is held on by two stout rubber bands, $\mathrm{B}^{1}$ and $\mathrm{B}^{2}$, and by changing this for one with a larger or smaller central aperture the sensitireness of the tambour can be at once decreased or increased. 


\section{CHAPTER III}

\section{A SINGLE CONTRACTION OF A FROG'S MUSCLE. ITS MODIFICATION} UNDER CHANGES IN THE EXTERNAL CONDITIONS

If a single stimulus of very short duration be applied to a muscle or its nerve, the muscle responds by giving a contraction of very short duration. This is termed a simple twitch, and is to be studied as in the following experiment.

Experiment 1.--Record a simple twitch of a muscle setting up the apparatus in the following way (see fig. 37). Connect one terminal of a battery $\mathrm{B}$ to one terminal of the primary coil $\mathrm{P}$ C, and the second terminal of this to the mercury key $\mathbf{k}$, and thence to the special break key $\mathbf{k}^{1}$, from the second terminal of which a wire is connected to the battery. The details of construction of the break key are shown in fig. 36 . A brass pillar D rotates

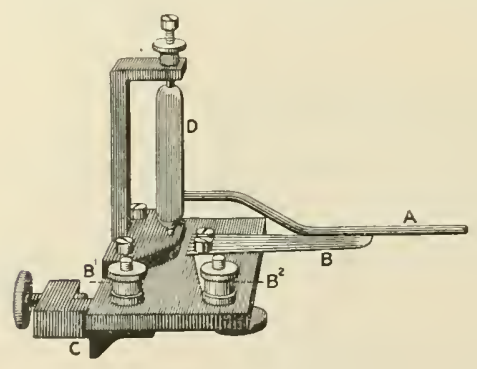

Fig. 36.-A Break Key. about a vertical axis upon two bearings, and to it is fixed a bent brass rod $\mathrm{A}$. To the metal bearings of $\mathrm{D}$ a binding serew $\mathbf{B}^{1}$ is connected, and this is fixed in an insulating base of vulcanite. A brass tongue B slightly curved upwards at its free end, is also fixed to the vulcanite base, and is connected to the second binding serew $\mathrm{B}^{2}$. The free extrenuity of $\mathrm{B}$ is slightly notehed to receive the $\operatorname{rod} \mathrm{A}$, and the two are liept firmly in contact by a screw, part of which is seen in the figure under the vulcanite base, which tends to force $\mathrm{B}$ upwards. If a current be made to enter at $B^{1}$ it will pass to $D$, thence along $A$ to $B$, and so ont from $B^{2}$. If $110 \mathrm{w} A$ be knocked on one side the current is broken directly $\mathrm{A}$ and $\mathrm{B}$ are separated. The drum having been covered and smoked is placed in position, and the arm $\mathrm{A}$ (fig. 37), fixed to a collar fitting on the axle of the eylinder, is brought into such a position that there is a well-blackened smooth piece of paper at the front of the drum, when the arm A touches the rod of the break key $\mathrm{K}^{-1}$. The two terminals of the secondary coil sc are connected to the two blocks of the Du Bois friction key $\mathrm{K}^{2}$, and to the remaining two terminals are connected the two wires of the electrodes E. A gastrocnemins-sciatic preparation is now excised, the femur fixed to the cork plate of the inyograph, and a fine thread tied to the tendon of the muscle, thus connecting it to the vertical arm of the crank lever. A small weight $\mathrm{W}$ is attached to the horizontal arm at a point near its axis, and the muscle so fixed that the writing lever is horizontal or points slightly downwards. The nerve is now laic across the wire electrodes, the key $\mathrm{K}^{2}$ being kept closed. A tmuing-fork F, or a chronograph, is arranged to write its tracing vertically 


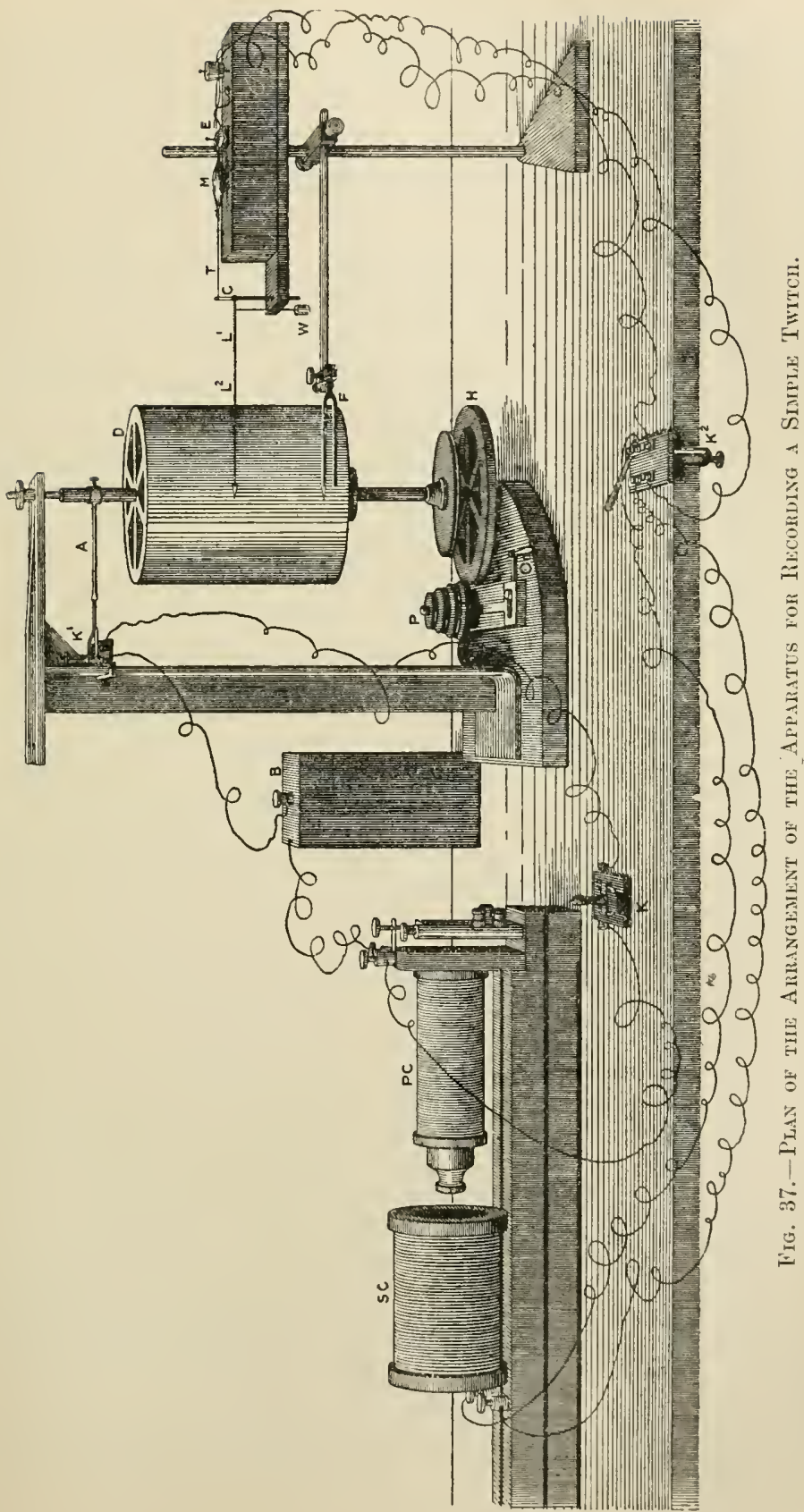


under the nyograph lever. Before either writing-point is allowed to tonch the snioked surface, the drum should be set in motion to see that the front of it rotates from right to left. The secondary coil is now bronght into sucl a position that naximal contractions are obtained when the key $\mathrm{k}$ is opened. The drum is now rotated until the rod $\mathrm{A}$ is a little in front of the arm of the break key $\mathrm{h}^{-1}$. Adjust the writing-point of the lever $\mathrm{L}^{2}$ to touch the drum surface whilst the tuning-fork $\mathrm{F}$ is not in contact. The key $\mathrm{K}^{1}$ is closed and then $\mathrm{K}^{2}$ is opened. The drum is next very slowly rotated by hand until the arm A breaks the key $\mathbf{r}^{1}$ and the shock thus produced in the secondary coil canses a twitch of the muscle, which is recorded as a vertical line on the smoked surface. The key $\mathrm{K}^{2}$ is again closed. Te now know that, no matter at what rate the drum be rotating, at the instant at which the arm $\mathrm{A}$ breaks the primary circuit the writing-point must be exactly opposite the vertical line just recorded. In other words, this vertical line represents the instant at which the stimulus will be sent into the nerve, i.e. it is the point of stimula. tion. Now rotate the drum through about a half-rerolution, set the tuningfork vibrating, and bring its writing-point in contact with the surface. Close the key $\mathrm{k}^{1}$ and open $\mathrm{k}^{2}$. Set the drum revolving by switching the friction wheel below the coned pulley $\mathrm{P}$ into contact with the wheel $\mathrm{H}$, the arm $\mathrm{A}$ breaks the contact of $\mathrm{n}^{-1}$, a stimulus is sent to the nerve, and the muscle contracts. As soon as the writing lever has returned to rest, the drum is stopped. This takes as a rule about half a revolution. The kev $\mathrm{K}^{2}$ is closed and the writing-point of the tming-fork removed from the surface. The writingpoint of the lever is once more bronght accurately on to the abscissa line, and the clrum rotated so that a horizontal line is recorded on the drum. This is the zero-abscissa line.

The drum is again rotated till the writing-point is brought to the line marking the point of stimulation, when the lever is depressed until it cuts the time tracing. In a similar way vertical arcs are drawn opposite the following three points: (1) the point at which the tracing leaves the zeroabscissa line; (2) the highest point of the curve; and (3) the point when it regains the abscissa line. One or two of such curves shonld be taken, and the curves given by different muscles should also be recorded. A tracing by a hyoglossus preparation is especially useful. This may be stimulated directly, for which purpose one electrode wire is wound round the pin fixing the hyoid cartilage to the cork plate, and the other may be attached to the bent pin passing through the tip of the inuscle, or it may be passed directly through the tongue from side to side. This wire should be very fine. The paper may now be removed from the drum, a note of the nature of the experiment may be written upon it by a finely pointed pen, and it may then be fixed by drawing it through a dish of ramish,' afterwards allowing it to dry.

In this way the curve of fig. 38 was obtained. The point of stimulation is marked at $a$, and $b, c, d$ are the other three points mentioned above. The rate of vibration of the tuning-fork is 200 per second. The muscle from which it was obtained was a hyoglossus, and the writing-point magnified the movement of the muscle three times. The curved lines $a a^{\prime}, b b^{\prime}, c c^{\prime}$, and $d d^{\prime}$, written while the smoked surface was stationary, are taken for the purpose of making the time measurements more accurately. The curve is seen to fall naturally into three parts : -

1 A very convenient varuish consists of 250 c.c. of best white-hard rarnish to which 1 litre of methylated spirit and 10 c.c. of castor oil are added. This dries quickly and gives a dull surface to the tracing. 
(i.) From the point of stimulation, $a$, to the point of commencing contraction, $b$. This is the LATENT PERIOD. During this time there is no change of length. The chronograph tracing $a^{\prime} b^{\prime}$ shows that this occupied $\frac{2}{200}$ ths of a second, i.e. 01 sec. This method does not give accurately the true measurement of muscle latency. It is too high. More accurate measurements by specially designed methods show it to vary from 003 to .008 sec. for frog's muscle. There are several reasons why a measurement by the above method cannot give the true result. In the first place, a muscle does not contract simultaneously all over, but the contraction starts at some one spot and then spreads in a wave-like manner over the rest of the muscle. Following an excitation at one spot, the fibres in that position contract, but do not at first lead to a movement of the recording lever, but rather to a stretching of the remainder of the fibre both above and below the point contracting. This is because the parts which have to be moved possess some inertia, and the part whose earliest movement we wish to record is not united to the lever by a rigid connection, nor is its upper end rigidly fixed, but at both ends muscle tissue is interposed.

As muscle is elastic the first result of the contraction of a part of a fibre is a stretching of the neighbouring parts, and movement will only be communicated to the lowest extremity when either the whole of the fibre has commenced to contract, or when the increase of tension by the stretching has been transmitted through the elastic fibre to that extremity.

(ii.) From the point of commencing contraction $b$ to the highest point of the curve $c$. This is termed the PERIOD OF CONTRACTION. The curve is for about the first third convex to the abscissa line, which means that the rate of the contraction is gradually increasing. This rate of contraction is at first very slow, as seen by the acute angle which the first part of the curve makes with the abscissa ; it then rapidly increases, as shown by the increasing inclination to the abscissa, and very soon reaches a maximum rapidity. From this, again, there is another change in rate, this time in the reverse direction, for the curve becomes concave to the abscissa line, and gradually, shortening becomes slower until at last it ceases, when the tangent to the curve becomes parallel to the abscissa line. The time occupied by the writing point in travelling from $b$ to $c$, as shown by the piece of time tracing $b^{\prime} c^{\prime}$, was $\frac{15}{200}$ ths of a second, i.e. $\cdot 075$ sec.

(iii.) The third portion of the tracing is from the highest point $c$ to the point $d$, at which the lever again reaches the abscissa line. This part is called the PERIOD OF RELAXATION. The terminal point, $d$, is often a difficult one to determine with any accuracy, because the lever does not come instantly to rest; but, as it always possesses some 
inertia, it oscillates for a time about a mean position which it ultimately reaches. The difficulty therefore is purely instrumental, and should be reduced to a minimum by working with as light a lever as possible. It is particularly marked when the relaxation process is carried out very rapidly, and is completely absent when, from any cause, the time is prolonged. An examination of this part of the curve shows practically the same changes as the preceding portion, though in the reverse order. It is at first concave, and then, after an intermediate portion in which the change of curvature is but slight, it becomes convex to the abscissa line. These changes mean that at first the rate of relaxation increases slowly, then more rapidly, until a maximum rate is attained, and from this gradually diminishes until relaxation ceases. The length of time occupied by this process, in the curve of fig. 38 , is seen, by measuring $c^{\prime} d^{\prime}$, to be $\frac{15}{200}$ ths of a second, or

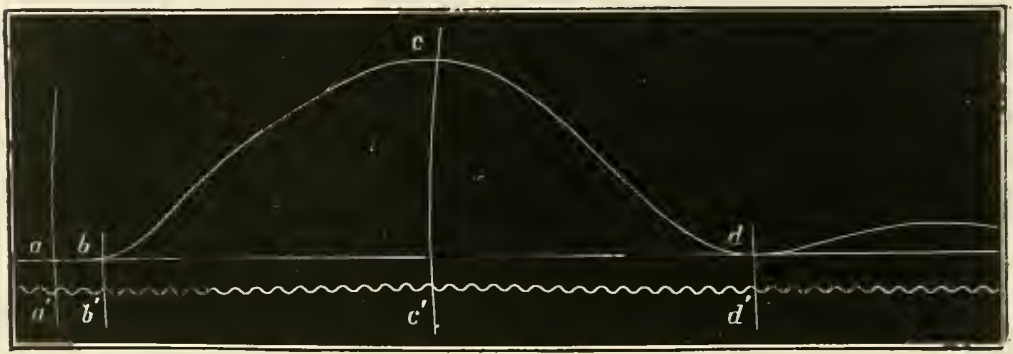

Fig. 38.-Isotonic Twitch of a Hyoglossus Muscle. Time Tracing, 200 per sec. Magnification, 3 (I.e. the Vertical Ordnate represents 3 times the AMOUNT OF SHORTENING AT THAT INSTANT).

$.075 \mathrm{sec}$; but this time measurement is not to be regarded as quite so accurate as the two preceding - it is probably estimated a little too high.

By adding up these three time measurements it is seen that for this twitch the total time occupied was 16 sec.

So far we have been mainly occupied in a study of the curve with regard to its time relations, but there are other points which the curve can teach us. In the first place the height of the curve will tell us the amount of the shortening that took place. The height of $c$ from the abscissa line is found to be exactly $20 \mathrm{~mm}$; and as the magnification of the movement was 3 , the amount the muscle contracted was 20 $\frac{20}{3} \mathrm{~mm}$. The length of the muscle when loaded by the lever was $28 \mathrm{~mm}$. Consequently the muscle contracted $\frac{20}{3} \times \frac{1}{28}$, i.e. nearly a quarter of its whole length.

We may in the? next place estimate the amount of work per formed by the muscle during its twitch. This is given by the product 
of the load lifted into the height through which it is moved, or w $=\mathrm{L} \%$. In this experiment the load, including the lever, was adjusted to be 2 grms. Hence the total work was $2 \times \frac{20}{3}=13.33 \mathrm{grm}$. $\mathrm{mm}$.

This work was effected by the muscle in the time .075 sec. Hence the mean rate at which the muscle worked during its contraction was $13 \cdot 33$ 075

The load was not, however, retained at the highest point, but

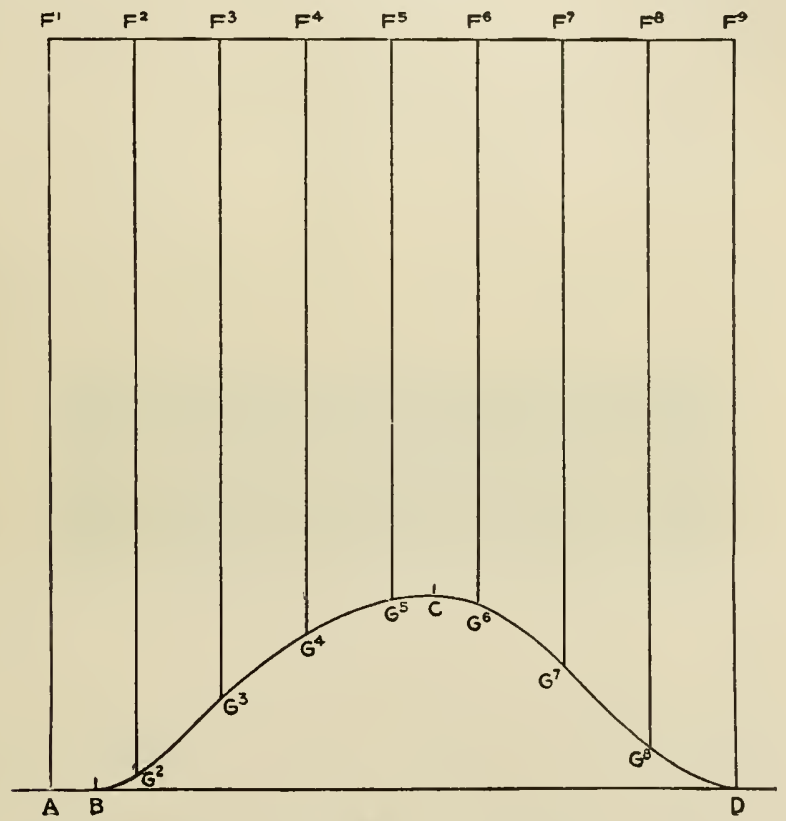

Fig. 39.-To Illustrate the Meanivg of the Curve of Fig. 38.

was allowed to fall again, and the lever ultimately came to rest at exactly the same level as at the start. Therefore, in falling, the load performed exactly the same amount of work upon the muscle as had been previously performed by the muscle upon the load. Moreover as in this particular example the time occupied in the relaxation happens to be identical with that of the contraction, the mean rate at which it was performed was identical with that of the former.

The exact meaning of the curve of fig. 38 or of any other curve taken upon the same principle by the graphic method will be rendered very evident by a study of fig. 39 . In this figure $A B$ C D is an exact reproduction of the curve of fig. 38. All measurements along A D represent 
time, and ordinates at right angles to this indicate for this particular example changes in length of the muscle. The muscle was $26 \mathrm{~mm}$. long; consequently $A F^{1}$ has been drawn at right angles to $A D$ and of three times that length $(78 \mathrm{~mm}$.) because the recording lever in tracing the curve magnified the shortening three times. $F_{1} F_{9}$ has been drawn parallel to $A D$ and divided at $F_{1}, F_{2}, F_{3} \ldots$ by a series of points which follow one another at intervals of four vibrations of the tuningfork. Hence $\mathrm{F}_{1} \mathrm{~F}_{2}, \mathrm{~F}_{2} \mathrm{~F}_{3}, \ldots$ \&c. each represent ${ }_{50}^{1}$ th of a second. Then $F_{2} G_{2}, F_{3} G_{3} \ldots F_{9} \mathrm{D}$ have been drawn parallel to $A F$, to cut the curve in $G_{2}, G_{3} \ldots$. In this way we are able to state that at the instant $\mathrm{A}$, at which the muscle was stimulated, its length was $\frac{1}{3} \mathrm{~A} F$.

$\begin{array}{llll}\frac{1}{50} \text { th of a second later its length was } & \frac{1}{3} \mathrm{~F}_{2} \mathrm{G}_{2} \\ \frac{2}{30} \text { ths } & \text { ditto } & \text { ditto } & \frac{1}{3} \mathrm{~F}_{3} \mathrm{G}_{3} \\ \frac{3}{50} \text { ths } & \text { ditto } & \text { ditto } & \frac{1}{3} \mathrm{~F}_{4} \mathrm{G}_{4} \\ \frac{8}{50} \text { ths } & \text { ditto } & \text { ditto } & \frac{1}{3} \mathrm{~F}_{9} \mathrm{D}\end{array}$

It should be remembered that in a great number of these graphic records the true interpretation to be placed on the curves is one similar

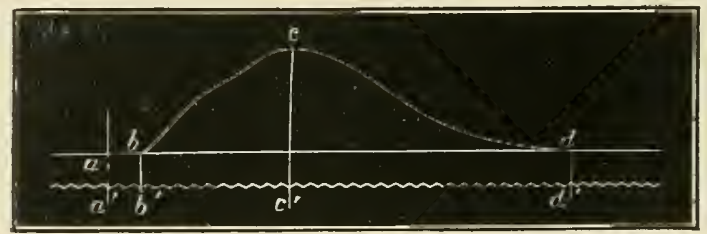

Fig. 40.-Sinple Twitch of a Gastrocnemivs. Time Tracing, 200 per sec. Magnification, 5.

to the above. As a rule, however, we shall find it sufficient to take into account only changes in length, width, \&c., according to the movement which is being recorded.

For purposes of comparison fig. 40 is introduced. This is a curve obtained in the same way, but given by a gastrocnemius. It shows the same features as those of fig. 38 . The latent period is $0.01 \mathrm{sec}$; the period of contraction $0.05 \mathrm{sec}$; and that of relaxation $0.1 \mathrm{sec}$. The magnification in this case was five-fold.

In recording a single muscle contraction by the method we have just employed, there is one factor in the method which causes an inaccuracy in the result we are aiming at, and as it leads to several important considerations we must examine into it with some detail. This is that the recording lever must possess a certain amount of mass, and, as a consequence, is unable to follow alterations in length in an absolute manner, more particularly when those alterations are 
effected at some speed. That the mode of action of this factor may be made more clear, let us consider what happens during the twitch of a muscle to which a weight is directly attached. The force exerted by the muscle during its contraction acts directly on the weight, which it sets in motion, and produces an acceleration in it directly proportional to the force and inversely proportional to the mass moved. The result is that the weight gains a certain amount of kinetic energy by virtue of which it will continue to move upwards, even though the muscle force ceases to act upon it, until that kinetic energy is neutralised by the constantly acting force of gravity. This is exactly the condition, then, when the force of the contraction begins to diminish ; and if, as is usually the case, we are actually recording the movement of the weight, the record of the true alteration of length is distorted by the operation of this acceleration. But the acceleration introduces another alteration which is even of greater influence, for as the acceleration upwards increases, so more and more of the weight ceases to pull directly upon the muscle, i.e. its tension diminishes. The result of a diminution in tension is that the elastic force of the muscle comes into play and produces a shortening which thus interferes with the shortening process we are attempting to record. The greater the load pulling upon the muscle the more will the action of acceleration interfere with the record. In the second process, that of relaxation, acceleration again comes into play. At first the tension diminishes because the weight does not follow the rapid relaxation instantaneously, but with a certain delay. When the relaxation is, however, becoming slower, the weight is moving downwards with a certain velocity imparted to it by the action of gravity, and a time is reached when the weight is moving downwards with a velocity greater than the rate of lengthening of the muscle. The result is it acts upon the muscle and the tension begins to rise, increasing until it is equal to the weight plus that force necessary to stop the acceleration. The muscle is therefore stretched beyond its initial length, and then, when the acceleration of the weight is stopped, the excess of tension over load acts upon the weight which is once more lifted, acceleration imparted to it, and the whole process is once more repeated, until with a few more oscillations the weight at last comes into equilibrium.

These final oscillations are to be observed in all tracings, and fig. 41 is reproduced especially in order to show them. The muscle recording was the double hyoglossus and the magnification five times. The only load attached to the muscle was the recording lever, in this case made of a light straw of about $25 \mathrm{~cm}$. in length. The preparation was the same one that had been previously employed 
to give the tracing of fig. 38 , where we note that these oscillations are

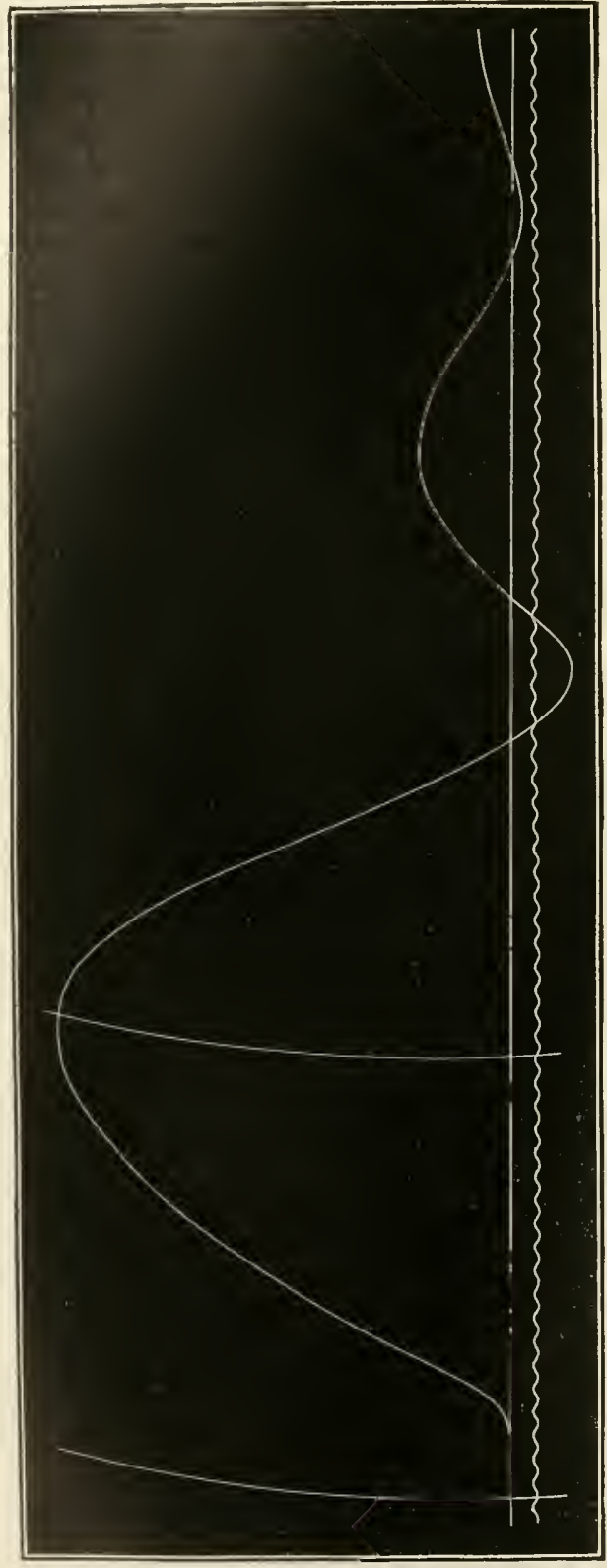

much less. To diminish the effects of acceleration we must always aim at employing recording levers of as small a mass as is compatible with the necessary rigidity. The lever used for fig. 38 was much lighter than that used for fig. 41.

It is, however, necessary for many experiments to study the contraction when the muscle is loaded. We have seen that if we apply the load directly under the muscle, acceleration mustcome into play with the result that the tension does not remain constant, but during the earliest part of the contraction period is increased, and during the later part and most of the period of relaxation it is below that of the weight, and at the end of relaxation it rapidly rises, and after a few oscillations ultimately reaches the initial tension. The part 
of the curve most markedly affected is the apex. Acceleration produces a greater distortion the greater the rapidity of the movement we are attempting to record.

In order to be able to record twitches under different tensions, and yet ensure that the tension remains practically constant throughout, Fick introduced what is known as the isotonic method. The end aimed at is to prevent acceleration of any part to be moved which possesses mass. This Fick obtained approximately by the arrangement shown in fig. 42. The weight $w$ is not applied directly beneath the muscle $\mathrm{M}$, but to a small pulley on the axis $\mathrm{A}$, and therefore much nearer the axis than the point of attachment of the muscle. In the particular lever drawn the muscle is attached to the point $P$ of the lever, $10 \mathrm{~cm}$. from the axis. This part of the lever consists of a light flat aluminium band, so that it is rigid in the direction in which movement takes place. The pulley has a radius of $5 \mathrm{~mm}$. Hence the tension on the muscle is $\frac{1}{20}$ th of the weight $w$. The movement of the weight upwards is also only $\frac{1}{20}$ th of the muscle movement, and consequently the bad effects of acceleration are diminished twentyfold. The curves of figs. 38 and 40 are taken with this lever.

There is a second important aspect from which we can study the physical manifestations of the processes underlying a muscle

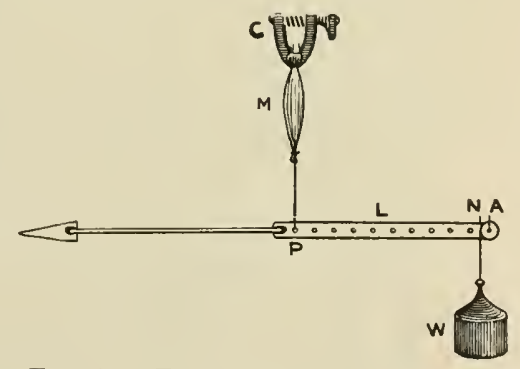

Fig. 42.-The Principle of the IsoTONIC METHOD.

contraction. This lies in answers to the questions: What happens if a muscle is prevented from shortening when it is stimulated? What variation in tension, if any, takes place? To investigate this problem Fick invented the isometric method, in which change of tension is recorded and change of length is practically prevented. The principle of the method is illustrated by fig. 43 . The muscle $x$ is attached above to a rigid support and below to a lever, $\mathrm{c} \mathrm{A}$, at a point near its axis, A. The lever is continued behind $\mathrm{A}$ in the form of a stiff spring, $\mathrm{A} B$, which rests on a rigidly fixed knife edge at $\mathrm{B}$. A light writing point,' c, is attached to record the movements of A C. When the muscle is stimulated it tends to contract and lift up $\mathrm{A}$ C, but this is resisted by the spring $\mathrm{AB}$, which is chosen of such a strength that the movement is very nearly prevented. The small upward movement of the lower muscle end is highly magnified by the long lever $\mathrm{A} \mathrm{D}$, and records the amount of bending of the spring $\mathrm{A} B$. If now the forces required to bend the spring so as to produce definite move- 
ments of the writing point are previously determined experimentally, any position of the writing point will be known to be caused by the

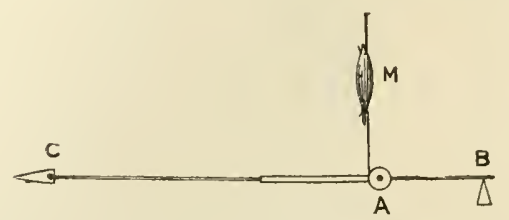

Fig. 43.-The Principle of the Isometric Method.

exertion of a certain force by the muscle. In this way, then, we may record variations in the force exerted by the muscle at different instants of its contraction.

Experiment 2.-Record an isometric twitch by means of the apparatus shown in fig. 44. The upper end of the muscle is to be fixed in the muscle forceps. The lower end by means of an S-shaped hook is attached to the lever $\mathrm{x}$ at a point near its axis. This axis consists of a stiff steel wire, $\mathrm{w}$, which can be clamped at any position by the screw $\mathrm{s}^{2}$. About $2 \mathrm{~mm}$. from the end of the axis the lever $\mathrm{x}$ is rigidly fixed perpendicular to it, and the projecting piece fits loosely into a small socket in the brass support A. The brass arm B carrying the axis can be adjusted horizontally and clamped in any position

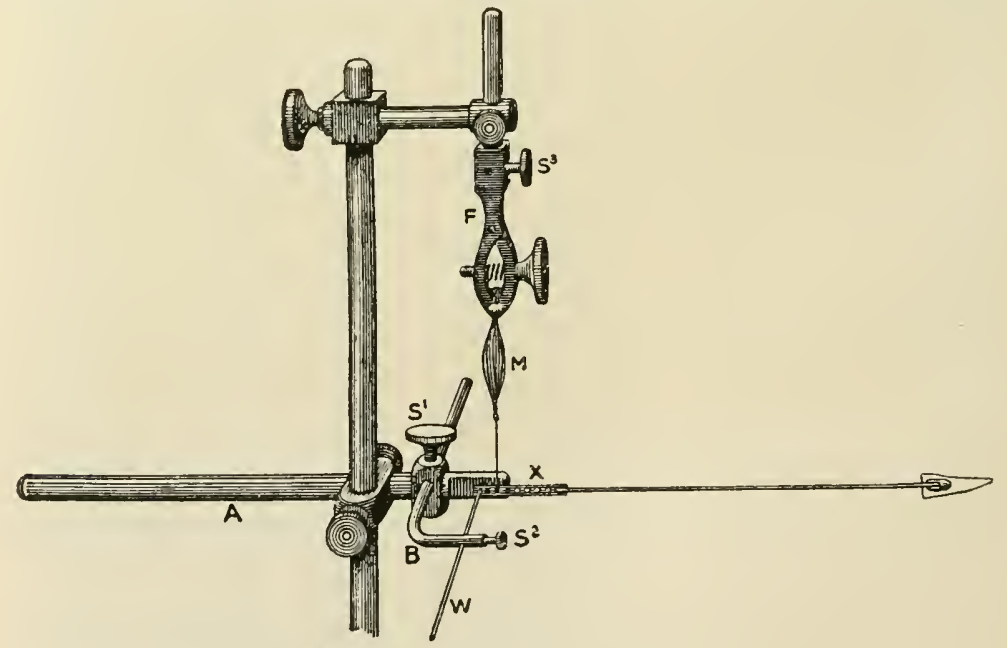

Fig. 44.-Mesche Attachei to an Isometric Lever.

by the screw $\mathrm{s}^{1}$, so that the free end of the axis just rests in its socket. Any rotation of the lever now produces a torsion in the steel axis, and this torsion is proportional to the amount of rotation of the lever. By hanging weights. on the lever and observing the torsions produced we shall then know that when that same displacement is produced by a muscle the tension exerted, which is exactly opposed by the torsion of the wire, is to be measured by the previously applied weights. Arrange the recording and stimulating apparatus. 
as for a simple twitch, p. 30, and then dissect out a muscle preparation. This should be the semimembranosus and gracilis (see p. 19). If we choose a small muscle which will only exert a small tension, a long piece of the torsion wire must be taken; if a powerful muscle a short piece. The muscle may be stimulated directly or indirectly. Record the twitch and tuning-fork tracing as before, and then determine the point of stimulation and draw an abscissa line. Next remove the muscle and to the lever attach a thread, which passes over a pulley held in the muscle forceps and to its free end hang a weiglit. This pulls up the lever and the writing point. By rotating the drum draw a line parallel to the zero abscissa. Now hang on a larger weight, and so obtain a second line, and by a series of these evaluate the movements of the writing point:

The form of curve obtained by this method is seen in fig. 45 . These curves were all taken from the same double semimem-

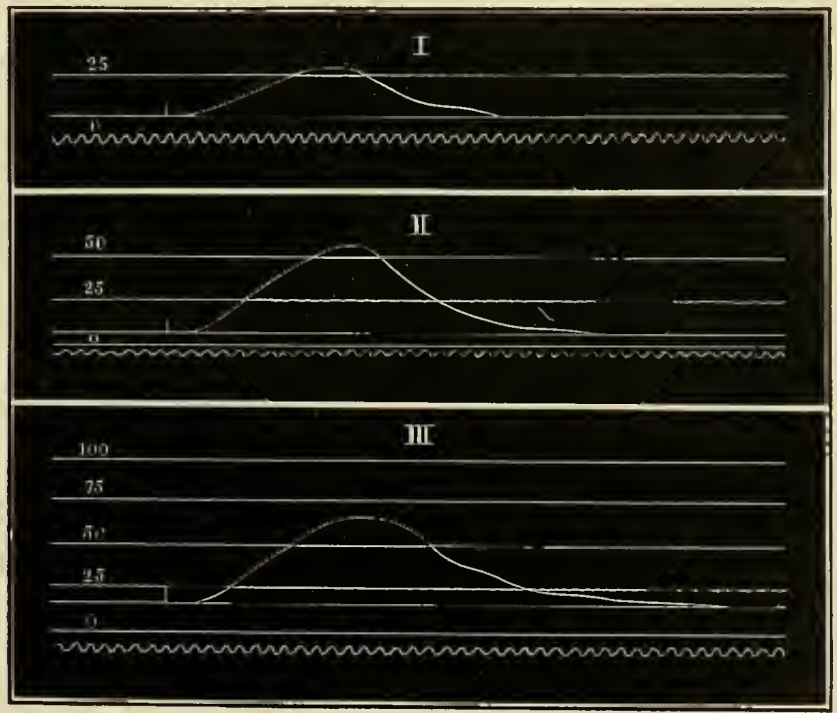

Fig. 45.-Three Isometric Twitches with Different Initial Tensions. The. Numbers on the Left indicate the Number of Grams required to Bring the Lever to the Level of the Corresponding Horizontal Line.

branosus and gracilis preparation with maximal stimuli, the only alteration in the conditions of the three experiments being with regard to the initial tension of the muscle. The horizontal lines indicate the amount of displacement of the lever by given weights, which are expressed in grams.

The general form of the curve is seen to closely correspond to that of an isotonic twitch, but measurement shows a few important differences. There is a latent period, a period of rising tension, and one of falling tension. The apex of the curve is seen to be rounded. 
The rise of tension is seen to be, on the whole, fairly uniform in rate, being slow at the commencement, and near the apex. Undulations on the curve are instrumental in character, and are due to the extremely disadvantageous position from which the lever is moved.

The measurements of such curves should be alranged in tabular form, as in the following table:-

\begin{tabular}{|c|c|c|c|c|c|}
\hline & $\begin{array}{c}\text { Initial } \\
\text { Tension } \\
\text { in Grams }\end{array}$ & $\begin{array}{c}\text { Maximum } \\
\text { Tension in } \\
\text { Grams }\end{array}$ & $\begin{array}{c}\text { Latent } \\
\text { Period } \\
\text { in Sees. }\end{array}$ & $\begin{array}{c}\text { Apex Time } \\
\text { in Sees. }\end{array}$ & $\begin{array}{c}\text { Total Time } \\
\text { in Sees. }\end{array}$ \\
\hline I & 0 & $30 \cdot 4$ & $\cdot 005$ & $\cdot 057$ & $\cdot 117$ \\
II & $5 \cdot 7$ & $55 \cdot 6$ & $\cdot 006$ & $\cdot 06$ & $\cdot 142$ \\
III & $\mathbf{1 6 \cdot 7}$ & $66 \cdot 7$ & $\cdot 007$ & $\cdot 065$ & $\cdot 170$ \\
\hline
\end{tabular}

From this table it is seen that the maximum tension attained during a twitch becomes greater when the initial tension is raised. With rise in initial tension the latent period, apex time, and total time all increase, but the greatest increase is in the period of falling tension.

By comparing these three measurements with those given for an isotonic twitch (pp.33-36) it is seen that the apex time for an isotonic twitch is longer than for an isometric, but that the period of relaxation is practically the same as the period of decreasing tension.

The aim of an isometric twitch is to be able to record the tensions a muscle is able to exert at each instant of a twitch carried out when it is prevented from shortening. It is important to recognise that the methods employed for this purpose can only give us an approximate result. This is due to the fact that we cannot prevent the muscle fibres from shortening, at any rate in part. When a muscle is stimulated the whole length of each fibre does not commence contracting at the same instant, but one part is first affected, and from this, as a centre, a wave of contraction travels along the whole fibre. As a result the part which first contracts exerts an increased tension upon the rest of the muscle fibre, which it stretches, and at the same time this extension allows it to contract. We could get a better solution to the problem if we could simultaneously stimulate the whole length of each muscle fibre, so that all its parts commenced contracting at the same instant.

\section{THE METHOD OF AFTER-LOAD}

In our study of the muscle twitch up to this point we have mainly been dealing with contractions carried out whilst the load on the muscle was as nearly as possible constant. There is, however, another 
method which very commonly obtains in many of our bodily movements, in which the muscle is under a low tension until it commences to contract, and then, only, experiences a rise of tension. We can imitate such a process by the plan of supporting the weight, and only allowing it to act on the muscle when it has already begun to contract. This is called the method of after-loading.

Experiment 3.-To examine the nature of a twitch under such circumstances arrange the apparatus as for taking a simple twitch. Prepare a gastrocnemius and attach it to the modified simple lever shown in fig. 46. This consists of an ordinary simple lever, but to the frame carrying the axis is fitted a stout brass plate, $B$, which runs parallel to the writing lever but not vertically under it. The end of this plate has a piece which projects under the lever and carries a screw, s. This can be screwed up so that the tip of the screw can support the metal part of the writing lever at any level. Load the muscle, $e . g$. with a load of $20 . g$., which, preferably, should be applied

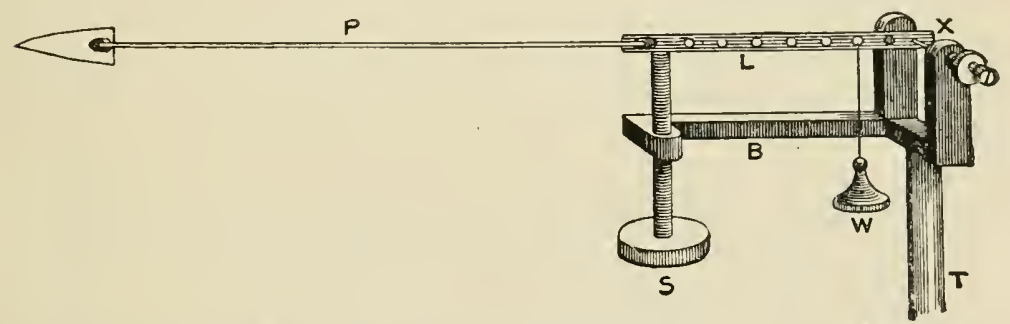

Fic. 46.-Arrangement of Simple Lever for Recording by the Method of AFTER-LOAD.

by a proportionately heavier weight attached nearer to the axis. Screw down the screw so that the whole load is carried by the muscle, and bring the writing lever to a horizontal position. Now record a simple twitch. Next screw up the screw to support the writing lever, so that the writing point is placed at the level of the apex of the curve just taken. Record from this position another curve. It will be found that the muscle still raises the lever. Paise the screw $\mathrm{s}$ once again until the level of the writing point is at the summit of this second curve, and again record a twitch. Repeat the process two or three times more.

Fig. 47 gives a series of curves obtained in this way. They are taken from a gastrocnemius in the manner described. The very striking and highly characteristic feature of these curves is that though the weight is supported at a level which it just reached at the height of a previous contraction, yet it is further raised when the muscle is again stimulated. Under such conditions, then, the muscle contracts to a greater degree than when freely loaded. As was to be expected, the latent period is longer in this second case, and, as the tracings show, becomes still further prolonged as the height of support of the weight is increased. The time measurements show for the four successive curves here reproduced $\cdot 01$ sec., ' 035 sec., 042 sec., 
and 065 sec. respectively. Two things are happening in these later twitches which account for this difference. In the first place, the muscle is taking in any 'slack' there may be. Secondly, and

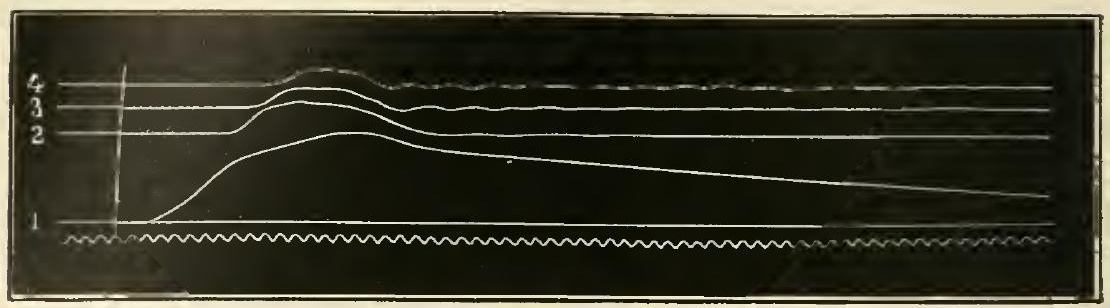

Fig, 47.-Twitches Taken teder the Princtple of After-loading.

more important, it is gradually increasing its tension until it is able to lift the load. The first part of such a twitch is therefore isometric ; but beyond a certain point it suddenly becomes isotonic, and its shortening is then registered.

\section{ALTERATIONS IN THE SIMPLE TWITCH BROUGHT ABOUT BY VARIOUS CONDITIONS}

I. The Influence of Temperature.-The differences in a simple twitch, brought about when the same muscle is at different temperatures, may be studied in many ways. If we are recording the twitches by a crank lever the muscle may be laid upon a metal base arranged so that it can be heated. Thus, in one form, the base is hollow so that water at different temperatures can be circulated through it. In another a stout metal wire is soldered to it, which is immersed in water at different temperatures, and so its temperature raised or lowered as required. In another form the muscle is suspended in a small moist air chamber made of hollow metal walls through which water is circulated. The chamber is completely closed except by a small orifice at the bottom through which the thread passes, attaching the lower end of the muscle to the recording lever. One of the most convenient forms is shown in fig. 48. This is to be employed for the purpose in the following experiment:-

Experiment 4.-Arrange the apparatus as for taking a simple twitch. Fit a cork c (fig. 48) tightly on to the lower end of the metal L-piece A B C. A weight $w$ is hung round the little pulley $D$ of the recording lever, so that it rotates the lever upwards. A muscle preparation is then made and its lower end fixed firmly by a pin to the upper edge of the cork c. The best preparation for the purpose is a hyoglossus or a sartorius, but a gastrocnemius may also be used. The upper end of the muscle is then fixed by a fine 
thread to the lever. The tension on the muscle may be varied by altering the weight $w$. The electrode wires from the secondary coil are connected (1) to the pin fixing the lower end $\mathrm{M}$, and (2) by a very fine light wire, N, passing throngh the upper end of the muscle. With this apparatus each twitch of the muscle pulls the lever downwards. The magnification should be about threefold or a little higher. The temperature of the muscle can now be altered at will by bringing up a small beaker containing a fluid at the required temperature, so as to immerse the muscle and the vertical limb of the L-piece. The immersion fluid may be normal saline made with tap water instead of distilled water, though it is better to employ defibrinated ox-blood which has been diluted with four times its bulk of normal saline solution, for it is found that the cha. racter of the contraction is very markedly altered if a muscle be soaked in normal saline for any

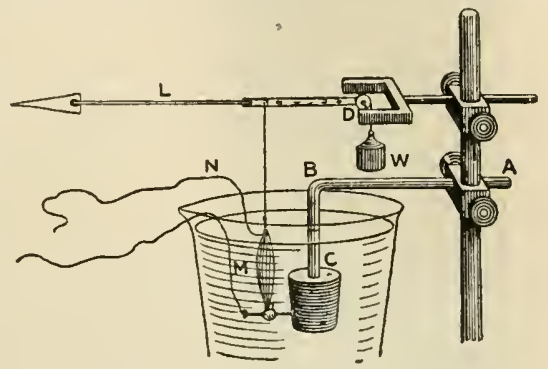

Fig. 48.-Apparatus for Varying the Teliperatures of a MUscle BY IMmersion. length of time. Having set up the munscle immerse it in fluid at about $0^{\circ} \mathrm{C}$. for three or four minutes, and then remove the fluid and record a twitch in the nsual way. Withont removing the writing point from the surface, again im. merse the innscle, having previously warmed the beaker of fluid by placing it in warm water until its temperature has risen to $5^{\circ} \mathrm{C}$. In three minutes remove the beaker and, if necessary, accurately adjust the writing point to the level of the previons abscissa, and then record a second twitch.

Repeat this for several higher temperatures, when a tracing similar to that of fig. 49 will be obtained. This tracing was given by a hyoglossus, the drum moving at a rapid rate. The various points in the curves should now be examined and the necessary measurements arranged in tabular form, as has been done in the following table for the curves of fig. 49 :-

\begin{tabular}{|c|c|c|c|c|c|}
\hline Temperature & Latent Periorl & $\begin{array}{c}\text { Contraetion } \\
\text { Time }\end{array}$ & $\begin{array}{c}\text { Relaxation } \\
\text { Time }\end{array}$ & $\begin{array}{c}\text { Height of } \\
\text { Twiteh in } \\
\text { mm. }\end{array}$ & $\begin{array}{c}\text { Mean Rate } \\
\text { of Work }\end{array}$ \\
\hline $7^{\circ}$ C. & 4.5 & 36 & 37 & 14 & \\
10 & $3 \cdot 25$ & 20 & 28 & 10.5 & 36 \\
15 & 3.0 & 15 & 21 & 9.25 & 41 \\
20 & 2.75 & 11 & 14 & 8.5 & 47 \\
25 & 2.25 & 9 & 10 & 8.5 & 58 \\
30 & 1.5 & $8 \cdot 5$ & 8 & 10.0 & 78 \\
\hline
\end{tabular}

In this table all the time measurements are recorded in $\frac{1}{20}$ ths of a second, and for the height of twitch the highest point of the tracing from the abscissa line in millimetres. The amount of contraction is therefore obtained by dividing these last figures by 3 , the amount of magnification. As the load was the same in all cases the total work in each case is proportional to the figures of the fifth column. 


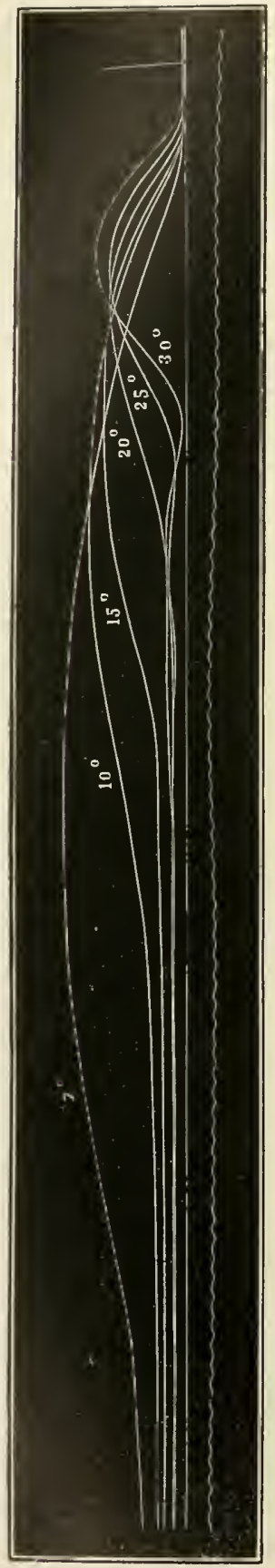

The mean rate of work is given in $\mathrm{grm}$ $\mathrm{mm}$. per second in the last column.

From the tracing and from the measurements given in the table the following points can be induced.

1. As the temperature rises the latent period, as measured by this method, gradually decreases, at first with some rapidity and then more slowly. These experiments, however, do not prove that the true latent period is diminished, for, as above explained, there are several factors at play tending to make the measurement by this method too high. The differences can probably be entirely accounted for in this experiment by the increase in the rate of propagation of the muscle wave as the temperature rises.

2. The period of contraction becomes markedly shorter as the temperature rises: at first the rate very rapidly increases, but after about $15^{\circ} \mathrm{C}$., though still increasing, its rate of increase is much slower.

3 . The period of relaxation varies in the same manner, but to a more marked degree. When the temperature is low relaxation is slower than contraction, in many experiments very much more so than in this particular instance. At higher temperatures relaxation is carried out more rapidly than contraction.

4. Perhaps the most interesting point in the whole series is with regard to the height of the twitch. In this case the maximum height was at $7^{\circ} \mathrm{C}$, and the height diminished as the temperature rose to $20^{\circ} \mathrm{C}$, at which a relative minimum height occuried. When the temperature was still further raised the height of twitch began once more to increase, and tended towards a second maximum at about $30^{\circ} \mathrm{C}$. 
5. As the load was the same throughout the series of twitches, the total work performed in the several cases is proportional to the heights of lift, and therefore shows the same variations as those pointed out in 4 . If, however, we examine the work from the point of view of the rate at which it was performed we see from the figures of the last column that there is a progressive increase in the rate of working which is particularly marked when the relative minimum height at $20^{\circ} \mathrm{C}$. has been passed. These rates of work are calculated, as previously described (p. 35), by dividing the amount of shortening by the time of contraction and multiplying by the load, which in this instance was one gram.

For purposes of comparison in fig. 50 is reproduced a similar series where the gastrocnemius was employed. The ar'angement of the apparatus was slightly different, being the one figured on p. 112, the muscle being fixed in the position at which the heart is there

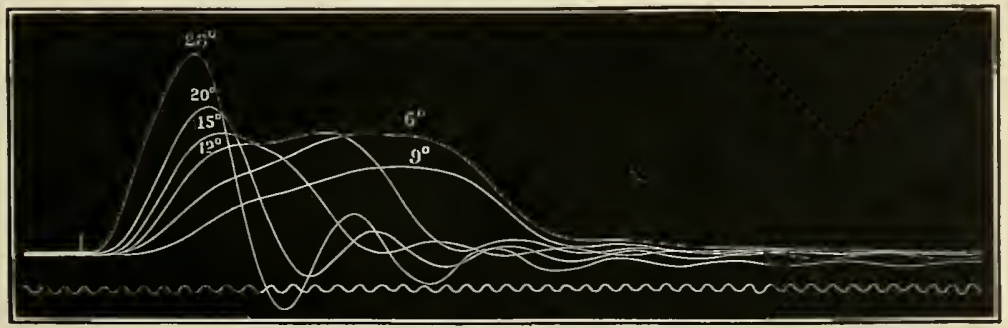

Fig. 50.-Twitches of a Gastrocnemios at Different Temperatures. Time Tracing, 200 per sec. Magnification, 5.

attached. The time tracing is 100 per sec., and the drum was moved at a rather slower rate. The general result is the same as in the previous example; but it is to be noticed that there is no relative minimum of height of twitch at $20^{\circ} \mathrm{C}$.

To complete our account of the influences of temperature upon the twitch there are one or two other facts known which do not appear in these tracings. It is found that if the temperature be still further lowered the total height of twitch is still further increased, and the relaxation much more markedly prolonged, and, as $0^{\circ} \mathrm{C}$. is approached, there is a rapid change in the direction of diminution in the amount of contraction, till at last none is produced. If, on the other hand, the temperature be still further raised the amount of contraction increases until about $32^{\circ} \mathrm{C}$. is reached: from this temperature the height rapidly diminishes, until the muscle goes into heat contraction at $34^{\circ} \mathrm{C}$. The twitches at the higher temperature are of the same total duration, although their height is less. 
II. The Influence of Load.-The effect of load upon the characters of a simple twitch are to be studied in three ways: (1) where the load is applied by the isotonic method; (2) where it is applied directly to the muscle; (3) where the load only acts on the muscle when it begins to contract.

Experiment 5.-Arrange the apparatus as for a simple twitch. Prepare a muscle and fix it in the isotonic lever as in fig. 4\%. The writing lever should be made as light as possible. The ordinary crank myograph lever could also be emploved, in which case the loads are to be applied at a point near to the axis. First record a simple twitch with the muscle weighted by the lever only. Then hang on a weight to the pulley by a thread. This extends the muscle, and the writing point must therefore be bronght back to its original level by raising the upper support of the muscle. Now record a second twitch. Increase the weight and bring the lever once more to its original level and record a third contraction. This may also be repeated until the amount of the contraction becomes very small.

Fig. 51 shows a tracing obtained in this way. The muscle was the hyoglossus. The loads in grams additional to the weight of the

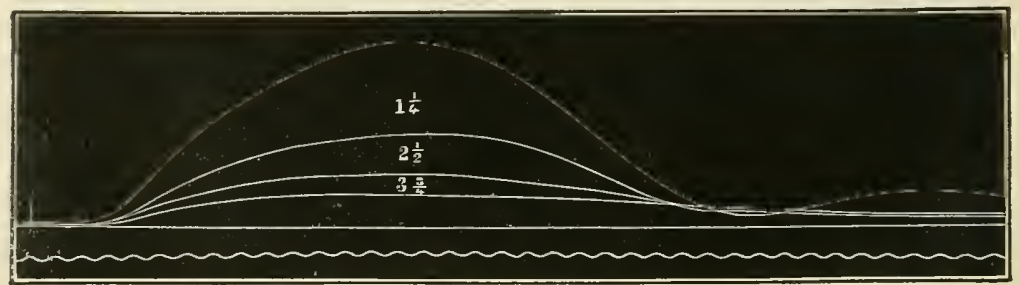

Fig. 51.-Twitches of a Hroglossus with Different Loads. Tine Tracing, 200 Per sec. Magaification, 3.

lever are written over the curves. Fig. 52 is a similar figure taken from a gastrocnemius.

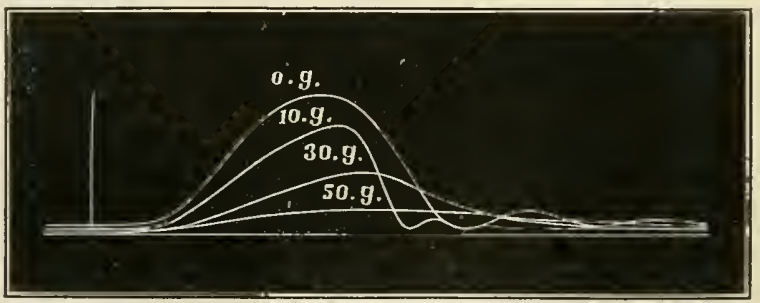

Fig. 52.-Twitches of a Gastrocnemios with Different Loads. Magification, 5.

The following effects are to be noticed :-

1. The latent period increases as the lond increases.

2. The length of the period of contraction also tends to increase. 
This is better seen in fig. 52, obtained from a gastrocnemius, than in fig. 51, from a hyoglossus.

3. The period of relaxation is at first markedly decreased. With higher loads it tends to increase again, often to a considerable degree.

4. The heights of the contraction progressively diminish as the load rises.

\section{THE INFLUENCE OF VERATRINE}

Experiment 6.-Destroy a frog's brain by pithing, leaving the spinal cord intact. Inject 5 minims of a 0.005 per cent. solution of veratrine dissolved in normal saline solution by the aid of a drop of weak sulphuric acid. Arrange the apparatus for taking a single contraction, but with the drum to rotate at a much slower rate (about $6 \mathrm{~cm}$. per minute). After about half an hour pith the spinal cord and dissect ont the gastrocnemius and sciatic, and fix the muscle in the myograph. In the preparation of the muscle care should be taken to aroid stimulating it or its nerve. Adjust the writing point to the blackened surface, and set the drum rotating. At any instant the muscle may be stimulated by opening the break key by hand. Note that the excitability is diminished, and a stronger stimulus than usual is required. The contraction will be effected quickly, but the relaxation is carried out very slowly, occupying some seconds. As soon as the muscle has completely relaxed, stimulate it once more. It will be found that the character of the twitch is completely altered. It is much mure rapid. One or two more contractions may also be recorded, and then the inuscle allowed to rest for a time. If it be then once more stimulated it will be found that the muscle has again returned to its previous state and the contraction is greatly prolonged.

The most satisfactory preparation to use for this experiment is the hyoglossus. This is attached to the lever in the usual way, and then a few drops of a 1 per 100,000 solution of veratrine in normal saline is injected into the large lymph sac in which the hyoglossus lies. If a very weak solution be directly employed in this way the muscle is ready for use almost at once, and the experiment never fails.

Fig. 53 represents two curves produced in such an experiment. Curve I is the first twitch, and curve II the sixth recorded. The first curve shows the characteristic effect produced. The early part of the period of contraction is effected as rapidly as in a normal twitch, but the latter part is very slow, lasting three seconds. The period of relaxation is 46 seconds. In curve II the total duration of the twitch has greatly diminished, viz. to 18 seconds, and another feature is present which is highly characteristic of veratrine; namely, that there is a double apex. The first contraction is carried out almost as rapidly as in a simple twitch, and it is then followed by a second contraction of very slow course, with rounded apex and showing a slow relaxation. The form of such a curve varies considerably in different instances. Sometimes the muscle may almost completely relax before the second contraction sets in, or again the second contraction may follow the first when that has reached its apex as in tracing $\mathrm{I}$. If 
the muscle be made to contract more frequently, and has not received too large a dose of veratrine, an almost normal contraction may be produced after a few stimulations.

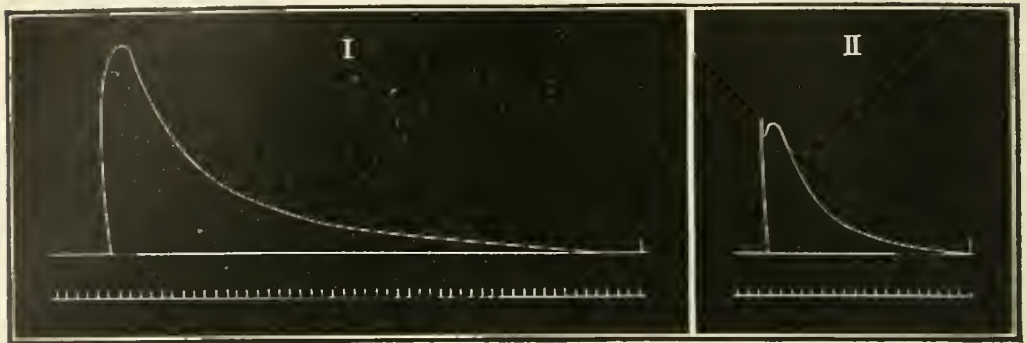

Fig. 53.-Two Twitches given by a Muscle Poisoned with Veratrine. Tiue ix Seconds.

\section{THE WORK PERFORMED DURING A TWITCH}

We have already pointed out how the amount of work performed during a twitch can be determined, and have studied some variations which occur on altering the conditions under which the contraction is carried out. It now remains to examine more completely the variations in the amount of work as the load is increased. The amount of work is represented by the product of the load lifted into the height of lift, so that if we simply require the total work performed we need only record the heights of the series of twitches.

Experiment 7.-Arrange the primary coil with a spring key for making and breaking the circuit by hand. Place a Du Bois key in the secondary with two fine wires for electrodes. Prepare the myograph lever and measure the distances of the point of attachment of the muscle and the end of the writing point from the axis. The writing point should be cut so that the ratio of the two is some simple multiple, e.g.5. Next prepare a gastrocnemius and attach one electrode to the fixed end, the other to the movable end of the muscle. Adjust the position of the secondary coil until it just gives maximal stimuli. Load the muscle with a tension of 40 grams, applying the weight at a point nearer the axis than the point of attachment of the muscle. The weight required to produce a tension of 40 grams will be found by multiplying 40 by the ratio between distance of muscle attachment to axis to distance of weight attachment to axis. This tension will elongate the muscle. The lever must therefore be brought back to the horizontal by altering the position of the fixed end of the muscle. Rotate the same a little to mark the level of the lever. Stimulate with a make shock recording the height of twitch on a stationary blackened surface. Increase the tension to 80 grams and repeat the process. Take a series of heights in this way, each time increasing the load by a fresh 40 grams. $^{1}$

1 The load chosen must depend on the size of the muscle. That of 40 grms. as here given is the most convenient for a medium-sized gastrocnemius. 
In this way a series of vertical lines have been recorded which give the heights of the several contractions. Now take a piece of squared paper and mark off these contractions in series, say $1 \mathrm{~cm}$. apart, measuring each contraction from the short horizontal mark to the apex. In this way a series of lines similar to those above $o x$ in fig. 54 will be recorded. Mark above each the weight with which the muscle was loaded at that contraction. The experiment from which

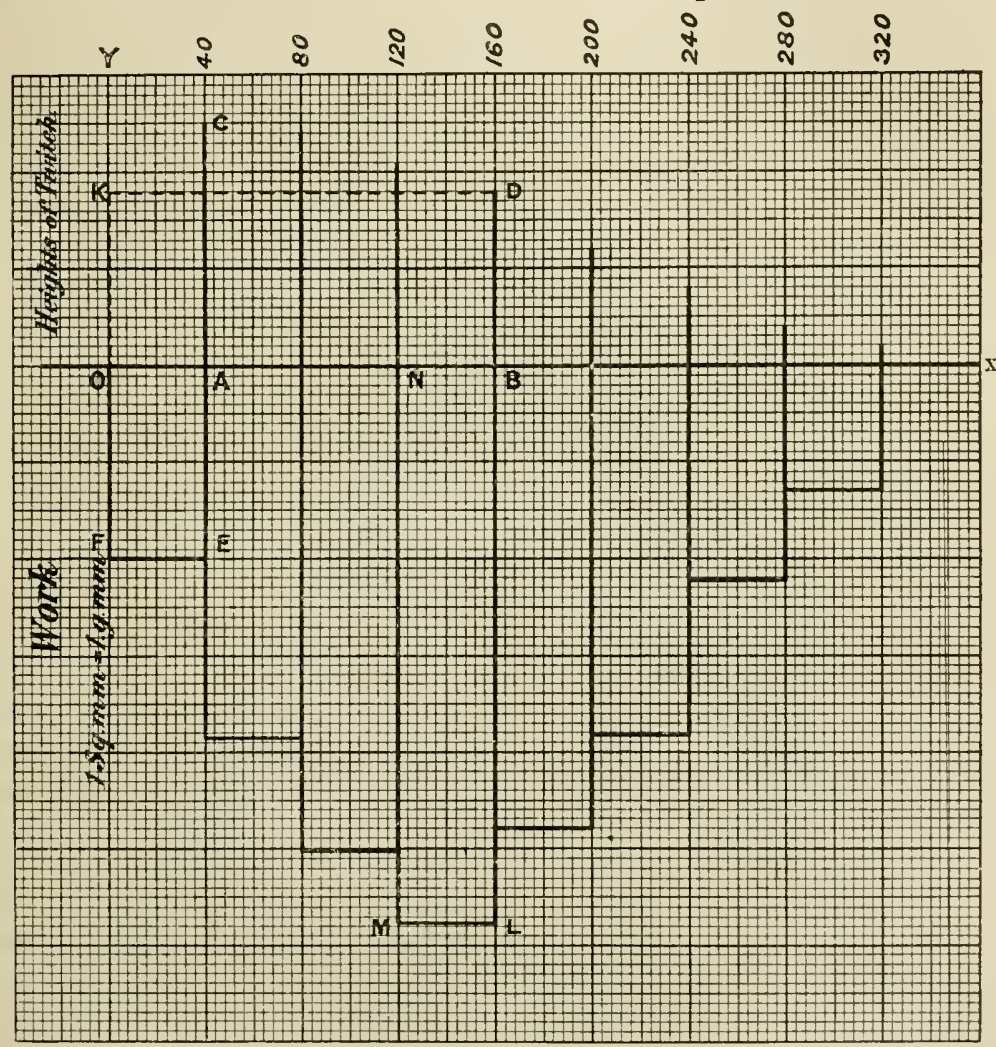

Fig. 54.-Work-diagrair of a Gastrocnemius for Single Twitches. Tim Ordinates above ox are Heights of Twitch ; the Rectangles below repreSent the Alounts of Work.

fig. 54 was drawn was carried out in this way. Distances measured along ox represent loads. Lines drawn parallel to o $x$ represent heights of contraction, and in this experiment they are magnified five-fold. Each $\mathrm{cm}$. measured along ox from o represents $40 \mathrm{grams}$. The work performed during one of the contractions, $c . g . \mathrm{B} \mathrm{D}$, is $! ? \mathrm{~B} \mathrm{D}$ $\times 160$ grm. mm., B D to be measured in $\mathrm{mm}$. As o 13 represents the 
load and $B$ D the height of contraction, the work is represented by $\mathrm{OB} \times \mathrm{B} \mathrm{D}$, i.c. by the rectangle $\mathrm{OB} \mathrm{D}$, and similarly for all the other contractions. These rectangles are not, however, readily comparable ; therefore proceed in the following manner. Determine the number of grm. mms. of work performed in each contraction and divide this by 10 ; measure off on the squared paper a line equal to the number obtained vertically under the contraction. Thus for the contraction в D, the work was $\frac{1}{3}$ о в $\times$ в D grm. mms. $=\frac{1}{5} \times 160 \times 18=576$. Therefore a line B L $57.6 \mathrm{~mm}$. was drawn as a continuation of D B. Then complete the rectangle $\mathrm{B}$ L is $\mathrm{N}$, which then contains 576 sq. mms., and therefore represents the total work performed, $1 \mathrm{sq}$. mm., representing $1 \mathrm{grm}$. mm. of work. It will of course be $\frac{4}{5}$ of the rectangle $O D$. In a similar manner the other rectangles were drawn and represent the other amounts of work for each contraction. The aim of this has been to represent each successive work by rectangles upon equal bases of $10 \mathrm{~mm}$., and therefore the heights are a measure of the work done and at once appeal to the eye.

We see directly that the amount of work performed at first increased rapidly as the load was increased, and reached a maximum at a load of $160 \mathrm{~g}$. From that point it decreased, but at a less rapid rate than it had increased, and at $320 \mathrm{~g}$, although the height of lift was only $\frac{2}{3} \mathrm{~mm}$., the amount of work performed was considerable. At $360 \mathrm{~g}$. the muscle only gave a scarcely perceptible lift.

This experiment also gives us a means of determining one other point in a muscle twitch, viz. the amount of load it is just able to lift when it is stimulated. In order, however, to get comparable results it is necessary to expresss this weight in a form for a definite amount of muscle. As it depends directly upon the transverse sectional area of the muscle, it is usual to express it in grams per sq. cm. of sectional area, and it is then spoken of as the 'ABSOLUTE FORCE' of the muscle.

We may gain this approximately from the preceding experiment if we know the length and transverse section of the muscle. In order to obtain these, the weight, specific gravity, and length were measured. These were: $\mathrm{W}=329 \mathrm{~g}$. S.G. $=1 \cdot 104, l=21.25 \mathrm{~mm}$. The load it was just unable to lift was $360 \mathrm{~g}$. Its volume $(v)$ is given by $\underset{\text { S.G. }}{W}=\frac{}{1 \cdot 104}=\cdot 298 \mathrm{cub} . \mathrm{cm} .=298 \mathrm{cub} . \mathrm{mm}$. Therefore its average transverse section $\frac{v}{l}=\frac{298}{21 \cdot 25}=14.02 \mathrm{sq} . \mathrm{mm}$. Hence the absolute force per sq. mm. of transverse sectional area was $\begin{gathered}360 \\ 14.02\end{gathered}=25.6$ grms.

Hence the absolute force per sq. cm. of sectional area was 2,560 grams. 
The absolute force of a muscle varies considerably in different muscles and in different animals. For mammalian muscles it is much higher than in the case of the frog's muscle. Determinations made upon the gastrocnemius have given results of as much as 8,000 to 10,000 grams per sq. $\mathrm{cm}$.

\section{THE PENDULUM MYOGRAPH}

A form of recording surface which has been largely employed for recording a single twitch of a muscle is the pendulum myograph, a simple form of which is shown in fig. 55 .

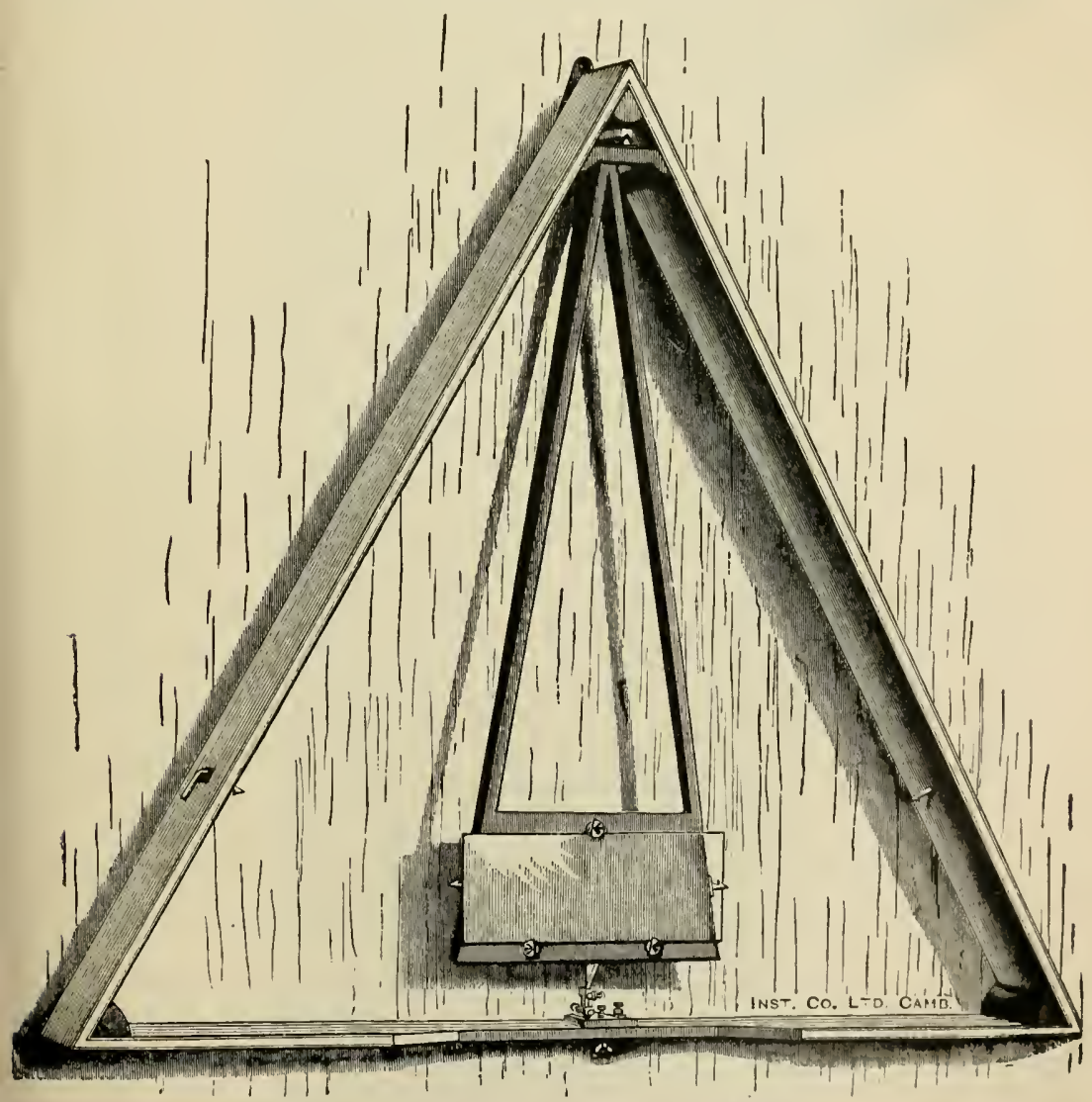

Fig. 55.--Simple Form of Pendulum Myograph,

It consists of a sheet of plate glass which is covered with glazed paper, and its surface is then smoked. This glass is held in a nupport at the end of 
a wooden pendulum swinging on two steel points at the upper angle of a large triangular frame. Clips are provided at either side of the frame by which the pendulum may be held at either side. Projecting from the lower end of the middle of the pendulum is a tongue of metal which breaks the contact of a special trigger-key the details of which are shown in fig. 56 . It

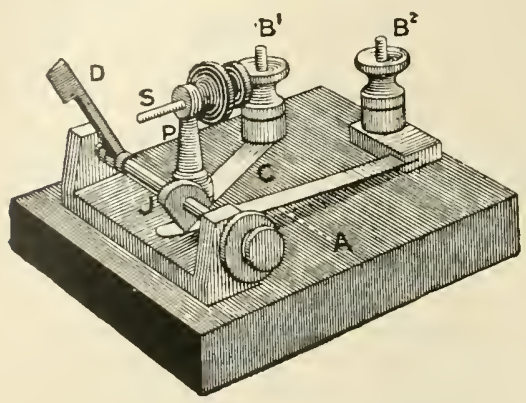

Fig. 56.-TRIGGER Ker. consists of a brass trigger $D$ fixed on an axis on which there is a sector $\mathrm{J}$ which is in contact with a brass spring $\mathrm{A}$, connected to a binding screw $\mathrm{B}^{2}$. The trigger is represented in the figure in an unstable position, so that if its upper end is moved slightly to the right, the spring $\mathrm{A}$ will bring it into contact with the end of the serew s. If, on the other hand, it is moved a little to the left, the spring will make it move downwards. The screw s fits in a pillar $P$ connected by a strip of brass $\mathrm{C}$ to a binding screw $\mathrm{B}^{\mathrm{i}}$. The screw is tipped with platinum and comes into contact with a platinum plate let into the surface of the trigger $\mathrm{D}$. If a current be sent throngh the key by the binding screws, it is closed only when the trigger $\mathrm{D}$ is in contact with the screw S. In that position the trigger is kept in contact by the spring. As soon as D is knocked to the left, the contact is broken and is not again closed if the trigger be carried beyond the nentral point in which it is drawn in the figure, being prevented from returning by the spring $\mathrm{A}$.

Experiment 8.-Take a simple muscle curve with this myograph. Insert the trigger key in the circuit of the primary coil, and place a Du Bois key in the secondary. Make a hyoglossus preparation and fix it to the recording lever, with electrodes for stimulating it directly. Close the trigger key, and with the pendulum hanging vertically bring the writing point to the surface; next bring the contact at the bottom of the pendulum to touch the trigger key while this is still pressed tightly to the screw s. Make a little vertical mark with the writing lever at this spot. This marks the point of stimulation. Now lift the pendulum until it is canght in the catch on the right, and having opened the Du Bois key, release the pendulum, which will then swing across and be caught in the catch on the opposite side. At the middle of the swing it will knock over the trigger, and the break shock in the secondary will this excite the muscle. Finally draw a zero abscissa line moving the pendulum by hand, and take a time tracing underneath.

Fig. 57 shows the form of curve thus obtained. The zero abscissa line is, of course, an arc of a circle of radius equal to the length of the pendulum.

The form of the curve is identical with those already studied in previous experiments. Time measurements should be made of the curve. 


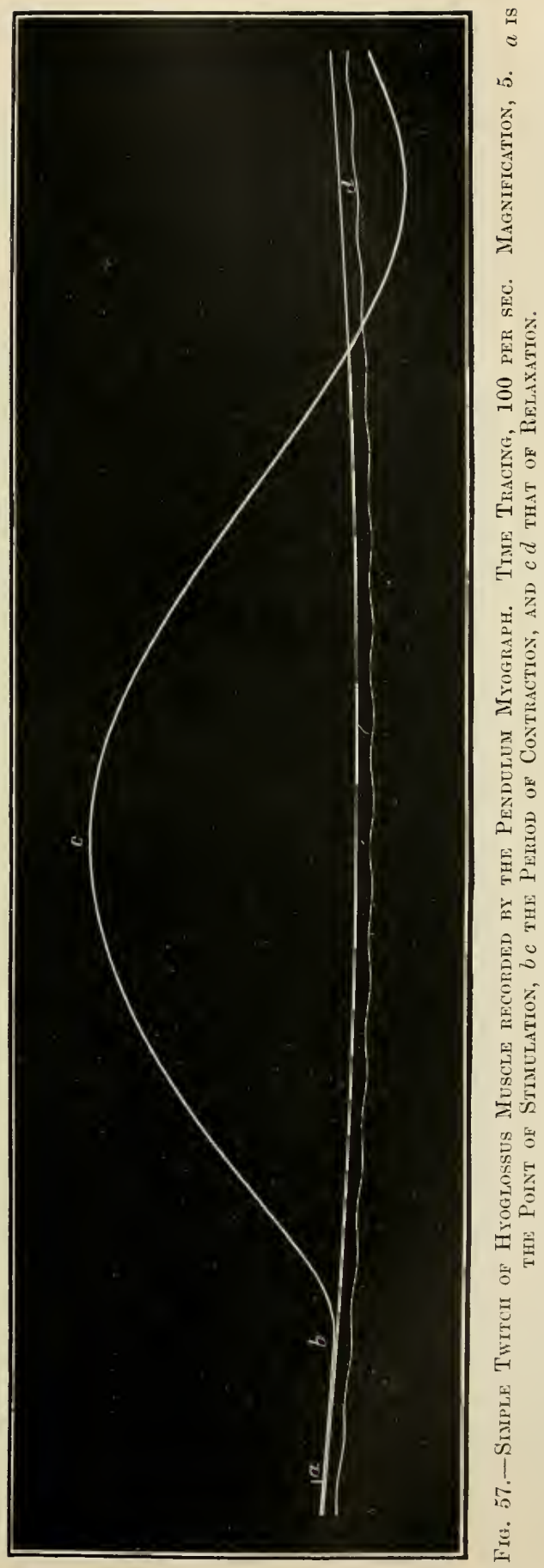


In order to study the nature and the production of a prolonged tetanic muscular contraction it is necessary to first examine the effect of applying a second stimulus to a muscle before the contraction caused by the first has ceased. This may be done by using some form of apparatus of the general principle of that described in the following experiment.

Experiment 1.-To the upper end of the shaft of the drum are fixed two brass collars, $\mathrm{s}^{1}, \mathrm{~s}^{2}$, which can be clamped in any position by means of screws. Held in the collars are two brass rods, $\mathrm{R}^{1}, \mathrm{R}^{2}$, terminating in flexible strips of brass, $\mathrm{x}^{1}, \mathrm{x}^{2}$. To the upright of the drum is clamped an insulating vulcanite base, on which is fixed a stiff brass rod, $\mathrm{x}$, to which a binding serew, $\mathrm{B}^{1}$, is connected. A second binding screw, $\mathrm{B}^{2}$, is attached at any convenient position of the metal stand of the drum. The arm $\mathrm{R}^{2}$ is now adjusted in the collar, so that the spring $\mathrm{x}^{2} \mathrm{jnst}$ touches the tongne $\mathrm{x}$, and the collar is rotated until a smooth piece of the blackened paper is opposite the writing point when the two are in contact. The second arm $\mathrm{R}^{\prime}$ is similarly adjnsted. The primary circuit is now arranged so that the current passes from the battery to one terminal of the primary coil, from the second terminal of this to the binding screw $\mathrm{B}^{1}$, and from the binding screw $\mathbf{B}^{2}$ back to the battery. Whenever either arm $\mathrm{R}^{2}, \mathrm{R}^{1}$ touches the metal $\mathrm{x}$ the circuit is then closed and travels through the coil to $\mathrm{B}^{1}$, through the metal $\mathrm{x}$ to the arm $\mathrm{R}^{2}$, and so up the shaft of the drum to its stand, and then through the binding screw $\mathbf{B}^{2}$ back to the battery. As the drum is rotated a little further the spring $\mathrm{M}^{2}$ is bent until it slips off the metal-piece $\mathrm{x}$ and the circuit is broken.

Make a nerve-muscle preparation (the muscle may be stimulated directly, in which case the nerve need not be prepared) and fit it to the myograph. Place the electrodes under the nerve (or so that the stimulus is sent throngh the whole length of the muscle) and move the secondary coil until the break shock will give a maximal contraction while the make shock is still ineffective. Bring the writing point to the surface and mark the two points of stimulation by very slowly rotating the drum by hand. As the first spring tonches the metal-piece an induced shock is produced in the secondary circuit, which, however, is not sufficient to stimulate the nerve or muscle. When rotated a little further the spring leaves the metal-piece $\mathrm{x}$, the current is broken, and the induced shock stimulates the nerve or muscle, the muscle contracts, and so records the point of stimulation. In a similar way the second point of stimulation is also recorded.

At first the second contact $\mathrm{II}^{1}$ should be fixed at some little angular distance 
from the first, so that the contraction caused by the first shall have had time to be completed before the second stimulus is sent in. The necessary angular distance will of course depend upon the rate of rotation of the drum. This need not be very rapid-abont 10 to $15 \mathrm{~cm}$. per second at the circumference. The contraction will then extend over about $1 \mathrm{~cm}$.

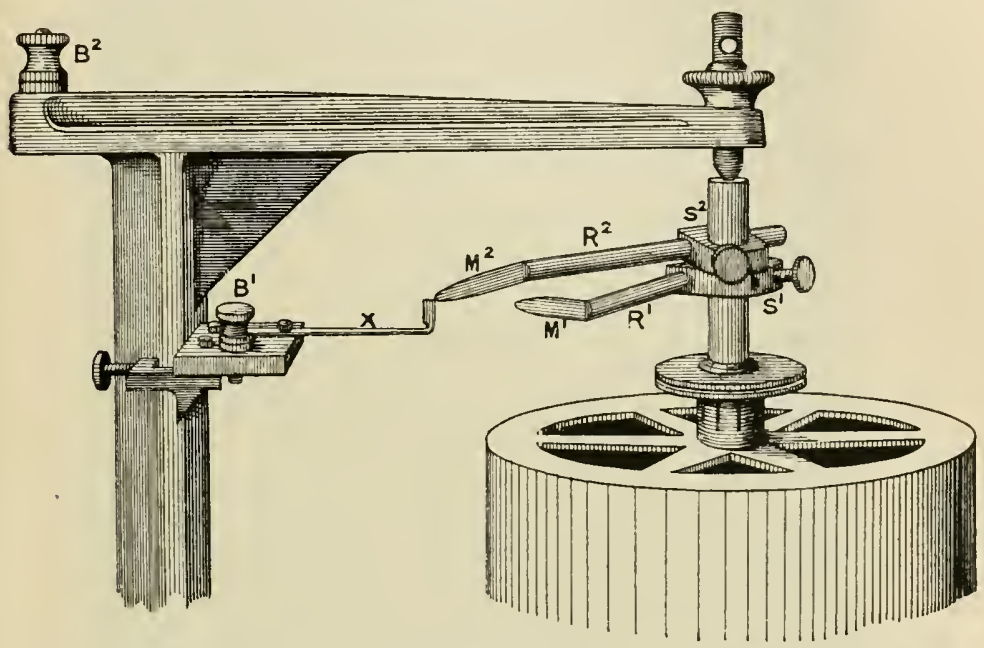

Fig. 58.-Arravgenent of Upper Part of Dirum for applyixg Two Stmuli to a Múscle.

of the paper. The double contraction may now be recorded with the drum in motion. Next move the second contact a little nearer the first and lower the myograph, so that it will record at a fresh level of the smoked surface. Mark the two points of stimulation as before, and then record the two contractions. Repeat this several times, on each occasion moving the second contact a little nearer to the first.

In this manner a series of curves will be recorded similar to those of fig. 59. In 1 and 2 the first contraction was finished before the second stimulation reached the muscle. The effect of the second stimulation is therefore a repetition of the first. A closer examination, however, shows certain differences. In the first place the second contraction is higher than the first, $9.75 \mathrm{~mm}$. and $9 \mathrm{~mm}$. for $1 ; 10 \mathrm{~mm}$. and $9 \mathrm{~mm}$. for 2 . Secondly, the total duration of the twitch is longer in the second than the first, $13.5 \mathrm{~mm}$. and $11 \mathrm{~mm}$. for 1 ; $15 \mathrm{~mm}$. and $13 \mathrm{~mm}$. for 2 . In tracing 3 the second excitation fell at about the commencement of the last third of the relaxation period of the first contraction and the commencement of the second contraction just before the lever reached the abscissa line. In 4 and 5 excitation occurred during the relaxation period of the first contraction; in 6 at about the apex of the first, and in 7 during the early part of the period of con- 
traction. We see that in these last five curves there is a summation of effect. The second apex is at a higher level than the first, and

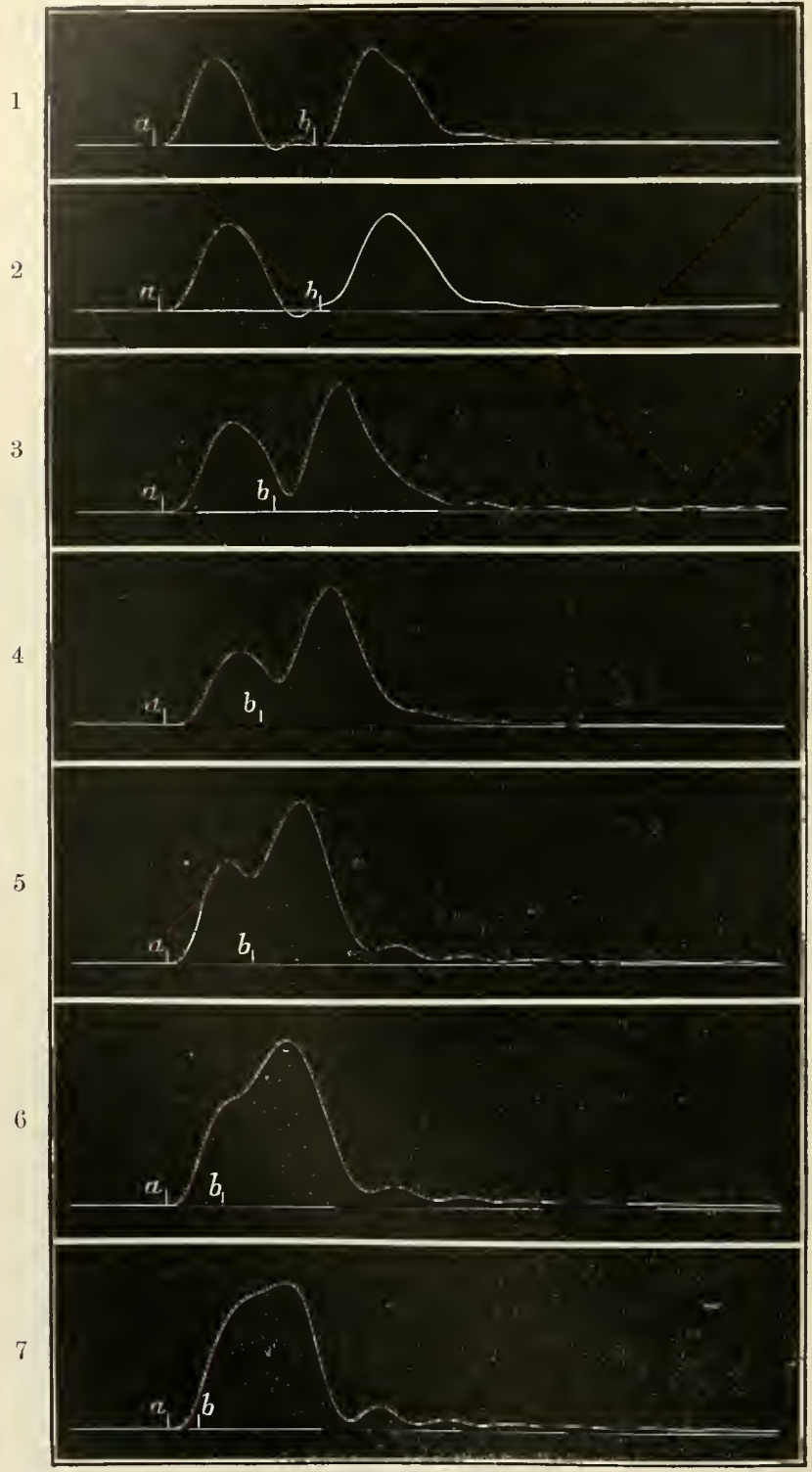

Fig. 59.-Erwect of Two Successive Stimuli, with Gradulim Dininishing Intervals, upon a Gastrociemies. 
the maximum summation effect occurred in curve 6 , where the height of the second apex is $17 \mathrm{~cm}$. as compared with 16.5 for the second apex of $5 ; 14.25 \mathrm{~cm}$. for that of $4 ; 13 \mathrm{~cm}$. for that of 3 ; and $9 \mathrm{~cm}$. for that of the first apex. As a general statement, it is found that the height of the second contraction is greatest when the instant of stimulation falls at the commencement of the last third of the period of contraction of the previous twitch. In connection with the height of the second twitch it is seen from curves 3 and 4 that when the commencement of the second contraction falls in the lower half of the relaxation of the first the height of the second contraction, as measured from the level at which it starts, is higher than the height of the first.

With regard to the time of the second contraction one other point of interest is found, viz. that the apex time of the second contraction is less than the apex time of the first, where by apex time is meant the total time elapsing from the instant of stimulation till the highest point of the curve is reached.

\section{THE GENESIS OF TETANUS}

- A study of the effect of applying two stimuli to a inuscle or its nerve, the time interval between the two being varied, naturally leads to a consideration of the effect which will be obtained by extending the number of stimuli.

To gain an answer to this it is necessary to have some form of apparatus which will give a series of stimuli at regular intervals, and further allow of an alteration of that interval. There are many forms of apparatus that will effect this, but the simplest consists of a flat steel spring which can be clamped in different positions, and whose free end is provided with a platinum wire by means of which a circuit may be closed with each vibration of the spring. The rate of vibration with a given spring depends, solely, upon the length of spring which is allowed to vibrate, and increases as the spring is shortened. To keep up the vibration of the spring it is usual to introduce an electromagnet which attracts it as soon as the circuit is closed. The method of fitting up the apparatus is shown in fig. 60. A $\mathrm{c}$ is the flat steel spring which can be firmly held in any position by the clamp c. At A a platinum wire is soldered to the free end of the spring, and this can close a circuit by dipping into the mercury $\mathrm{x}$ held in a vulcanite cup. $\mathbf{E}$ is an electromagnet held above the vibrating reed. In order to get regular vibration of the reed the electromagnet should befixed at a point about two-thirds of the length of the spring away from the clamp (i.e. nearer to $\mathrm{A}$ than its position, as shown in the figure). To fit up the apparatus connect one terminal of the battery 13 with the mercury cup by means of the binding screw $\mathrm{T}^{1}$. Connect the clamp of the reed by its binding screw $\mathrm{T}$ to one terminal, $\mathrm{E}^{1}$, of the electromagnet and the second, $\mathbf{T}^{2}$, to one terminal, $\mathrm{P}^{1}$, of the primary coil. The second terminal of the coil is then connected to the battery. Supposing now that the platinum wire A 
dips into the mercury, the circuit is closed, and consequently the electromagnet attracts the iron reed, the point of which is thus lifted from the mercury, and the circuit is broken. The reed then falls, the current is again made, and the cycle repeated indefinitely. Note that the general principle of the whole arrangement is precisely the same as that of the Neef's hammer attached to the inductorium. The only difference that we have is, that the length of the reed, and consequently the rate at which it vibrates, is easily adjustable, whereas in the Neef's hammer the rate of vibration is permanently fixed. In some ways it is rather more convenient to fix the reed so that it vibrates horizontally instead of vertically, as in fig. 61 . The platinum point is then bent downwards and the mercury cup overfilled, and so adjusted that the platinum point makes contact by touching the side of the mercury. By using it in this position there are fewer adjustments to be made when the

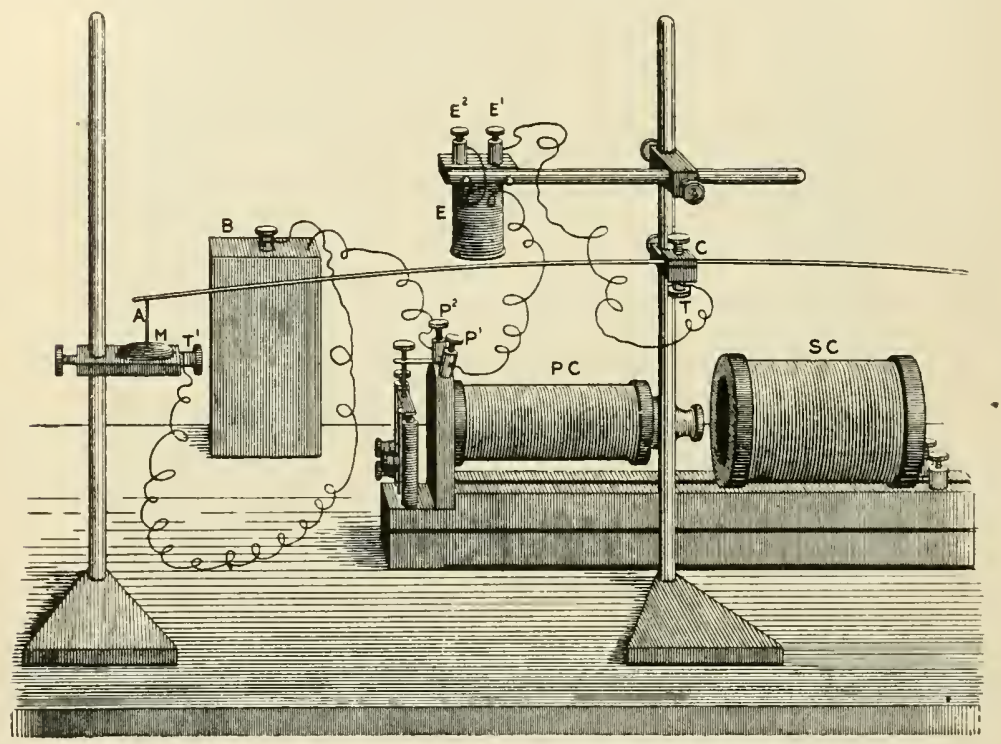

Fig. 60.-Mode of Fitting dP a Vibrating Reed.

spring has to be changed to \& fresh position, and it will be found that the spring vibrates rather more regularly. The wire connections are the same as in the previous case.

In using the reed each time the platinum dips into the mercury, a make shock is produced in the secondary; and each time it is lifted from the mercury a break shock is prodnced, so that there are two stimuli for each complete vibration, which are, moreover, of unequal value. This is a great disadvantage in the employment of the vibrating reed which can, to a certain extent, be overcome by so regnlating the position of the secondary relatively to the primary that only the break shocks are effective in producing a twitch. Even then, however, we must not consider the make shock as producing no effect, for it is found that a shock which in itself is insufficient to cause a contraction can produce an alteration in the nerve or muscle, which is 
shown in a difference in effect produced by a second stimulus which rapidly follows it.

The make shock (or break shock) may be cut out by using the key shown

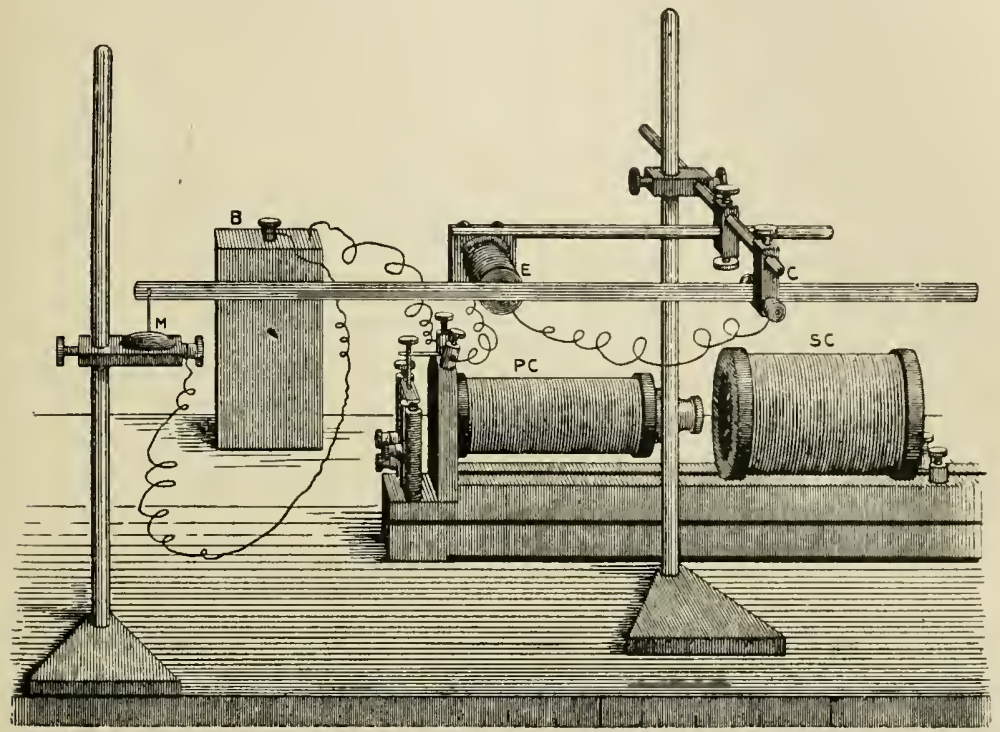

Fig. 61.-Reed arraxged to Vibrate in a Horizontal Plane.

in fig. 19, and fitting in the two notched wheels instead of the sectors. Alteration in the rate at which the stimuli are sent in can then be effected by altering the rapidity at which the key is driven.

Experiment 2.-Adjust the vibrating reed as just deseribed, at first clamping it quite at the end, so that it vibrates two to three times per second. Make a nerve muscle preparation and fix it in a simple lever or crank lever myograph, and having covered and smoked the drum arrange it so that it rotates at the rate of $3 \mathrm{~cm}$. per second. Adjust the secondary coil, using a Du Bois key as a short-circuiting key and placing the coil in such a position that it gives maximal shocks on break while the make shocks do not stimulate. Close the key in the secondary circuit, set the vibrating reed in action, bring the writing point up to the smoked surface, and allow the drum to rotate. Open the key in the secondary for about one to two seconds, and thus allow the shocks to reach the nerve. The inuscle contracts and its inovements are recorded. Stop the drum and next shorten the length of vibrating reed, and take a second tracing in the same manner as before. Take a series of tracings in this way, between each, making the reed vibrate a little faster by shortening it. When the reed has been sufficiently shortened the effect at last produced is a complete tetanus.

In an experiment carried out as thus described the series of tracings shown in fig. 62 were obtained. The time tracing in all cases was eight per second. The preparation was the gastrocnemius stimulated indirectly, and the magnification was threefold. In curve 1 the 


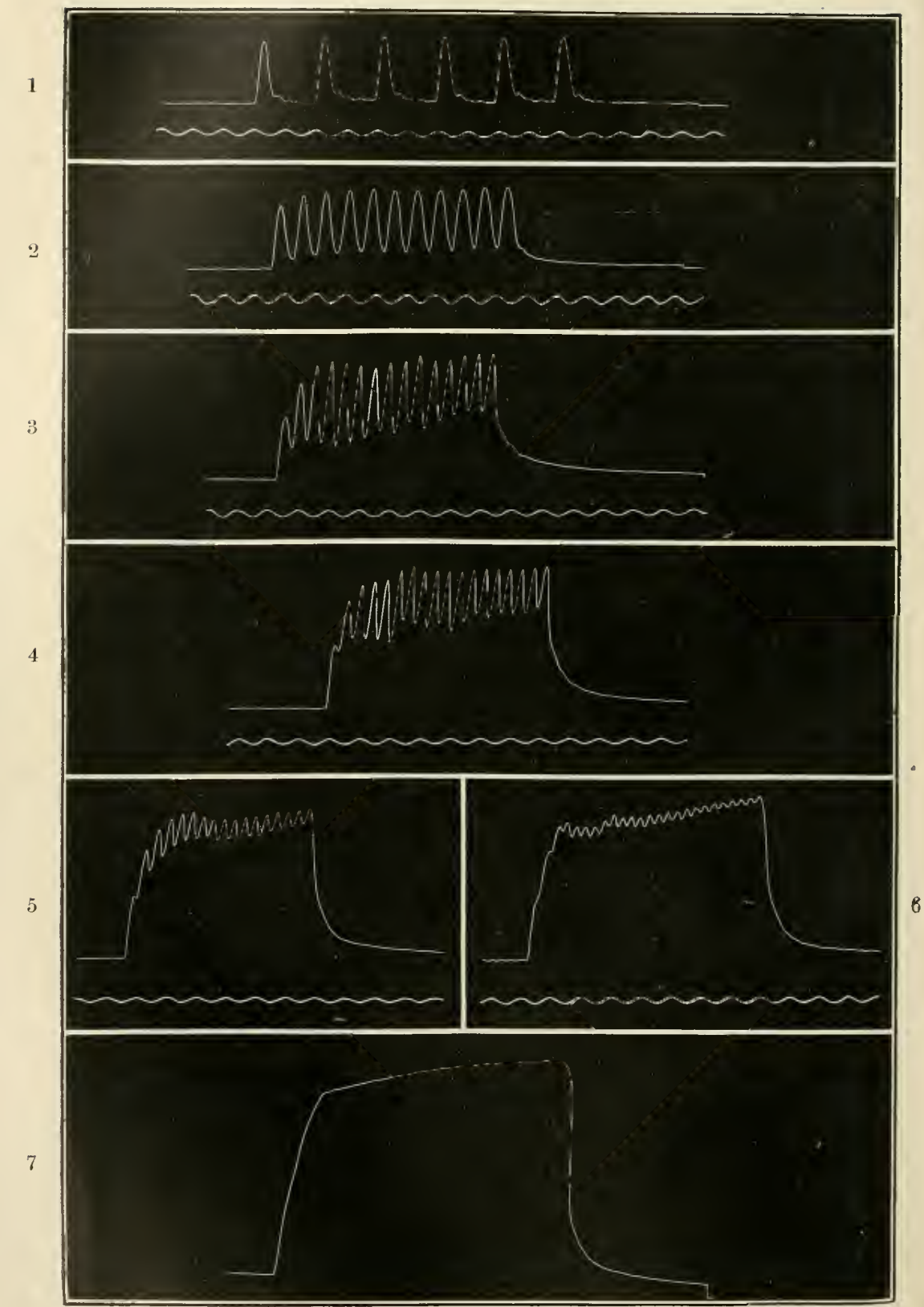

Fig. 62. - The Gradual Production of Tetands as the Rate of Stmullation was Increasen. 
rate of stimulation was four per second, and it is seen that each contraction is distinct. On measuring the heights of the twitches it will be found that the second is a little higher than the first, and the third a trace higher than the second, the last four all being of the same height. This 'staircase' effect, very slightly marked in this particular instance, is usually to be observed, especially if the preparation be very fresh and has not been injured during its removal. It shows that by a contraction a muscle is brought into such a state that an immediately following stimulus of the same strength evokes a more powerful response. That it is a question of increased capacity of performing work rather than an increase of excitability is indicated by the fact that it is to be observed when maximal stimuli are employed.

In curve 2 the rate of stimulation was increased to ten per second. We see that the first contraction was nearly complete before the second stimulus led to a fresh contraction. The lever did not reach the zero abscissa line, and some summation occurred. The third stimulus effected a contraction before relaxation was complete, and a fresh summation occurred; and so on for the later contractions, though after the fourth the maximum height of contraction did not increase.

In curve 3 the rate of stimulation was further increased to sixteen per second, and a much greater summation of effect is recorded. The second apex lies well above the first, the third above the second, but to a less extent, and the fourth above the third, though here the increase of shortening was only slight. From this the apices remained at practically a uniform level. An interesting point is to be noticed in this curve, viz. that the lowest points of the undulations of the curve become progressively at a higher level as stimulation proceeds. This effect is due to fatigue brought about by the number of contractions the muscle has been made to give. One of the effects of fatigue (see chap. v.) is found to be a marked slowing of the rate of relaxation, so that as this was produced in this instance the later stimuli began to produce their effects on the muscle before relaxation had proceeded quite as far as in the preceding contraction.

In curve 4 the rate was increased to 18.5 per second, and in 5 to 22 per second. The curves show practically the same points as those already described for 3 . The only difference, apart from the lesser excursion of the oscillations, is that there is more summation, and therefore both upper and lower apices are at a higher level than in the preceding curves.

Curve 6 shows a similar condition, though carried to a further degree. The line joining the upper apices is approximated to that 
joining the lower, and in addition one other feature is observed, viz that the amount of contraction progressively increases throughout.

In these last four curves it is to be noticed that the relaxations of the first few contractions progressively increase. In curve 5, for instance, the first relaxation is practicaily nil, the second $2 \mathrm{~mm}$., the third $3 \mathrm{~mm}$., the fourth $3 \mathrm{~mm}$, and at a later stage when fatigue begins to set in, they again diminish in amount, due to the cause already explained. The meaning of this increase in relaxation receives a certain degree of explanation in the light of the fact already

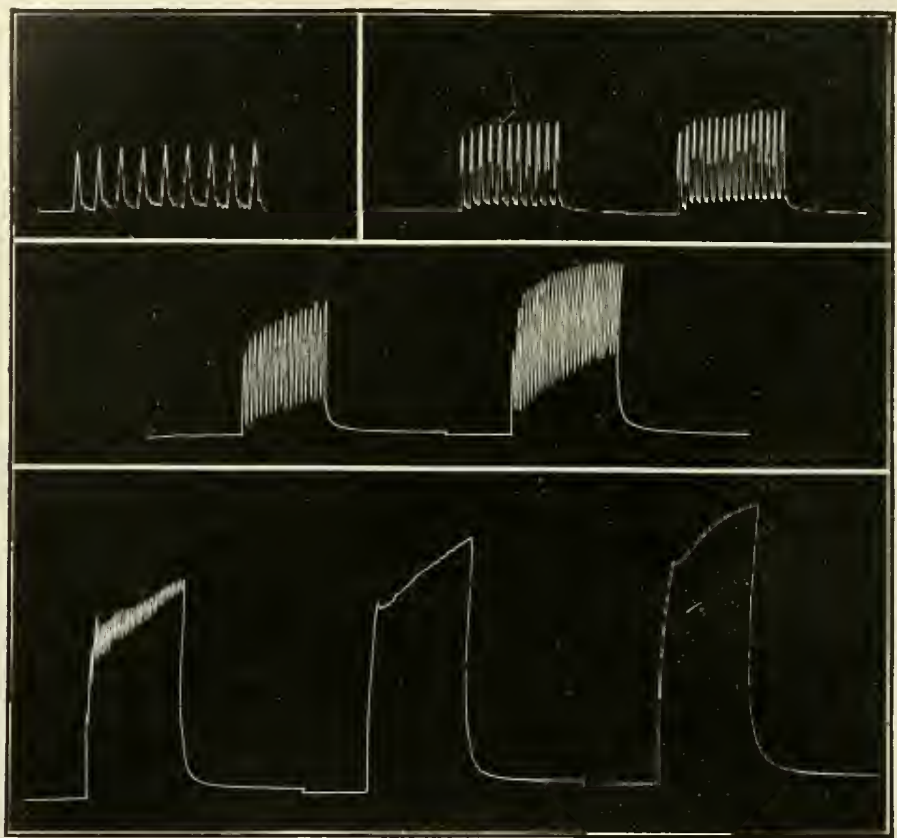

Fitr. 63.-The Genesis of Tetanus with slow Rotation of Recording Surface.

studied, that the apex time of the second twitch in a summation series is less than that of the first. The diminution of apex time in a series has also been found to extend to the third, and in favourable cases to a fourth or later contraction.

In the last curve (7) a practically complete tetanus is recorded which was produced when the rate had reached thirty per second. It is seen that the upper line of the whole curve very gradually ascends. There are slight wavy oscillations on this line, which, however, are not synchronous with the series of stimuli. 
In a similar manner repeat the experiment upon a hyoglossus. The experiment may be further modified by reducing the rate of rotation of the drum to $1 \mathrm{~cm}$. per second. In this way fig. 63 was obtained, the magnification being twofold. The figure brings out clearly the same points as those we have already studied in the preceding instance. With the slower rate of recording surface the way in which the lines joining the apices or bases of the successive contractions ascend is very clearly seen.

Take a tracing of complete tetanus employing the Neef's hammer of the coil for the purpose, when a tracing similar to curve 7, fig. 62, p. 62 , will be obtained.

Experiment 3.-To effect this, attach the two poles of a battery to the two pillars of the coil, and adjust the vibrating hammer until it works continuously. Insert a Du Bois key in the secondary circuit as a short-circuiting key. Excise a nerve-muscle preparation and fit it in a myograph, laying the nerve across the electrodes. Upon a slowly moving surface record the result of stimulation of the nerve for a short time. Repeat, varying the strength of the stimuli. 


\section{CHAPTER V}

\section{FATIGUE OF MUSCLE}

A PERFECTLY fresh muscle possesses in itself a certain store of potential energy in the form of chemical substances by whose decomposition the muscle is able to perform a definite amount of work, or exert a definite force. If the muscle be caused to contract and produce either a series of twitches or a prolonged tetanic contraction, this chemical energy becomes more and more used up, and the muscle passes into a state in which it becomes more and more difficult for it to respond to the stimuli sent to it. This difficulty is due primarily to the using up of the initial store of chemical energy, and secondarily to the accumulation of chemical bodies of the nature of waste products which obstruct the action of the physico-chemical processes which are the basis of a muscle's activity. In this state a muscle is said to be fatigued. We can study this condition of fatigue, from a chemical standpoint, and again we may study it by contrasting its performances while in this state with its behaviour under similar conditions when in a normal state.

To show how a single twitch becomes modified as the muscle passes into fatigue proceed in the following way.

Experiment 1.-Fit up the apparatus in a similar manner to that employed when studying the effect of two successive stimuli upon muscle (fig. 58), but remove the second contact $\mathrm{I}^{1}$, so that only a single stimulus is sent into the muscle with each revolution of the drum. The rate of rotation of the drum should be arranged so that a complete revolution occupies about two-thirds of a second. In this way the contact $\mathrm{r}^{2}$ by touching $\mathrm{x}$ closes the primary circuit three times in two seconds, and the induced shocks of the secondary circuit are at this rate. A muscle preparation is now got ready and fitted into a myograph, and the electrodes fixed in position. The secondary coil is so placed that make shocks are ineffective. The point of stimulation and an abscissa line are now drawn. It is of great importance that the writing point should in this experiment only draw a very fine line. The drum can now be set in rotation. With each revolution a twitch is caused which is recorded. After about ten to fifteen minutes the drum may be stopped, and the record examined. During this time from 1,000 to 1,500 
twitches will have been recorded, and unless the original contractions were great, and the writing point very fine, the lines will be found to have in many places largely obliterated one another. To obviate this it is best to only record each tenth or twentieth contraction, moving the writing point free from the surface during the intermediate twitches. In bringing it once more in position it is necessary that it should be brought back exactly to its first position, so that the point of stimulation is correctly placed. To carry this out with complete accuracy requires some mechanical means of adjusting the writing point to the surface.

Fig. 64 reproduces a series of curves obtained in this way. The first six curves are numbered in the order in which they were taken. No. 1 was the first twitch; 2 the sixth ; 3 the eleventh ; 4 the twentyfirst; 5 the thirty-first; 6 the forty-first; and the remainder at intervals of ten twitches. Curve No. 1 gives a typical simple twitch. Curve 2 is seen to differ from this in a few points :-(i.) It starts from a rather higher level. This is not of great importance in this instance for this difference is chiefly of instrumental origin. (ii.) Both the period of contraction and that of relaxation in 2 are rather longer than in 1. (iii.) On measurement the height of the twitch in 2 is found to be slightly higher than in 1 . On contrasting 3 with 2 these differences come out even more clearly.

After curve 3 the heights begin to gradually decrease, and the times of the different periods to increase. Thus the latent period gradually increases from 017 second to 03 second. The contraction time increases, though not:very greatly; but the chief change is in the period of relaxation, which is seen to become very greatly extended, so much so that the drum has revolved and a fresh stimulus been received before relaxation is complete, as seen by the fact that the next contraction starts with the lever at a higher level than in the previous curve. This condition of more or less permanent contraction is spoken of as contractur. If the experiment be carried on for a longer time it is found that the relaxation process to a certain degree recovers itself. Relaxation time slowly diminishes after about four or five thousand twitches, but never attains the previous speed seen when the muscle was fresh.

The condition of fatigue, then, is chiefly characterised by a slowing of the usual processes of a twitch, a diminution in height, i.e. in the total work performed, but most prominently by the prolonged time required for relaxation. The series of changes observed in fatigue may be to a considerable extent modified by variations of the external conditions. Thus, heat markedly accelerates the onset of fatigue. Load also hastens the production of fatigue; but as it aids the relaxation the condition of contractur is not so clearly seen. 


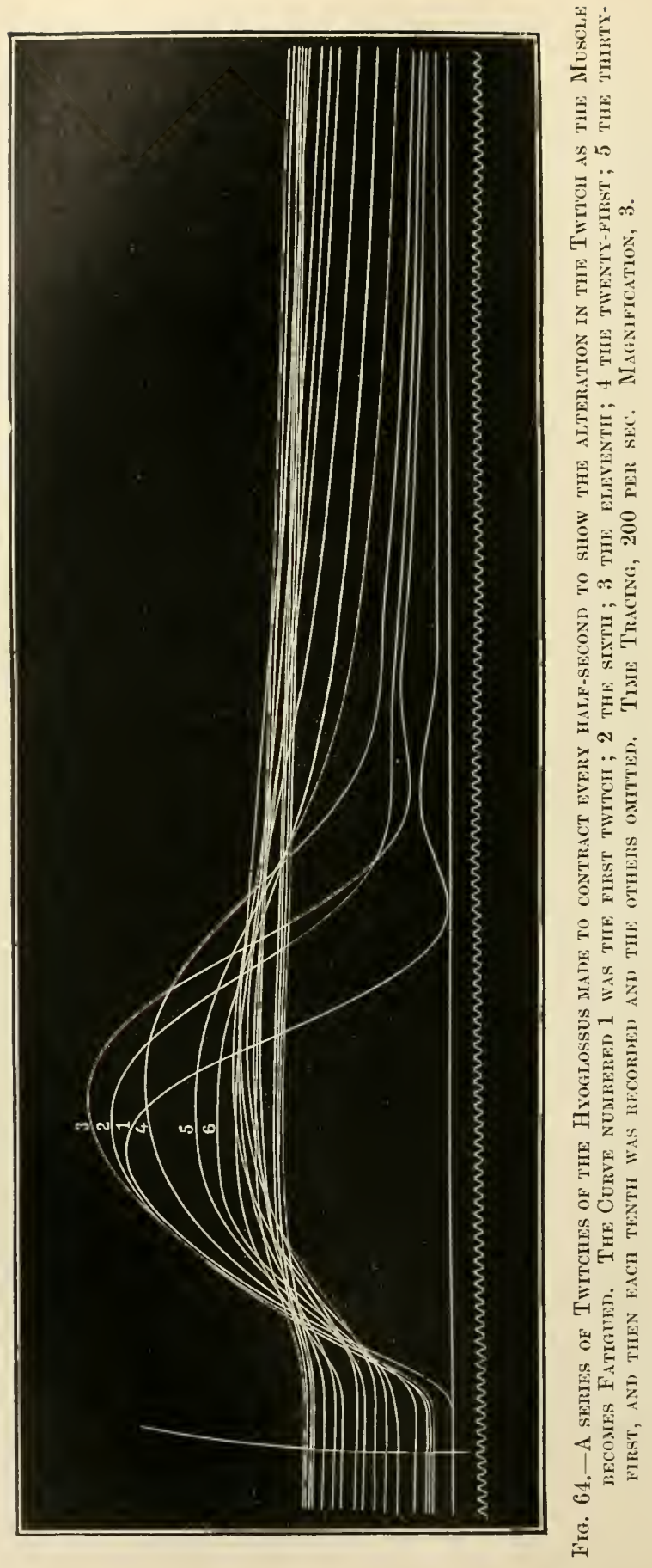


Next study the effect of fatigue in producing alterations in the height of contraction, i.e. in the amount of work it is able to perform with constant load, by the following method:-

Experiment 2.-Drive the cut-out key, fig. 19, from the shafting employed to drive the drum, so that it gives two shocks per second. Connect it with wires to give make shocks only. Fit up a chronograph to give a seconds time tracing. Dissect out the gastrocnemius or hyoglossus, and having fitted it to the myograph lever attach the electrode wires to stimulate directly, and then bring the secondary coil up to the primary until the contractions produced on each make of the primary circuit are maximal. Gear down the drum until it rotates at about the rate of $10 \mathrm{~cm}$. per minute. Bring the writing point to the recording surface, start the drum, and then open the short-circuiting key in the secondary circuit and record the contractions for about five minutes.

In this manner the tracing of fig. 1, pl. 1, was obtained. The tracing was given by a hyoglossus preparation which was loaded by the weight of the lever only. The heights of twitch only are recorded. It is seen that the first ten twitches give a typical 'staircase' effect, and from that point onwards, the heights gradually diminish to the end of the tracing, at first rapidly and then more slowly. The second point of interest is with regard to the line joining the basal points of the twitches. This is seen to gradually leave the zero abscissa line, as fatigue sets in, which is to be explained by the fact we have already studied in the previous experiment, viz. that elongation becomes greatly prolonged, and hence a new stimulus reaches the muscle before it has had time to completely relax. The time at which the succeeding stimulus reaches the muscles falls progressively at earlier stages of the relaxation. There is produced therefore a condition of contractur. On stopping the stimulations the muscle is seen to slowly relax, but had not regained its initial length when the tracing was stopped.

The muscle should now be allowed to rest for about ten minutes, and the experiment once more repeated, when a result similar to that of fig. 2 , pl. 1 , will be obtained. It gives as it were an epitome of the changes observed in the previous tracing. The changes in the twitches are brought about very rapidly instead of slowly, as in the first tracing. If this tracing be carried on for a very long time it will be found that gradually almost complete fusion is obtained, and then again the undulations become much more marked. This last change occurs when the late stage is reached in which the relaxation process begins to shorten again.

Experiment 3.-Stndy the effect of fatigue upon the tetanus curve by arranging the rate of the vibrating reed to just give complete fusion and record a tetanus by means of this, keeping the stimulation up until complete fatigue sets in. 
It will be found (fig. 65) that the contraction soon begins to diminish, at first with some rapidity and then more slowly as fatigue sets in. At $a$, fig. 65 , the stimulation was stopped, and it is seen that

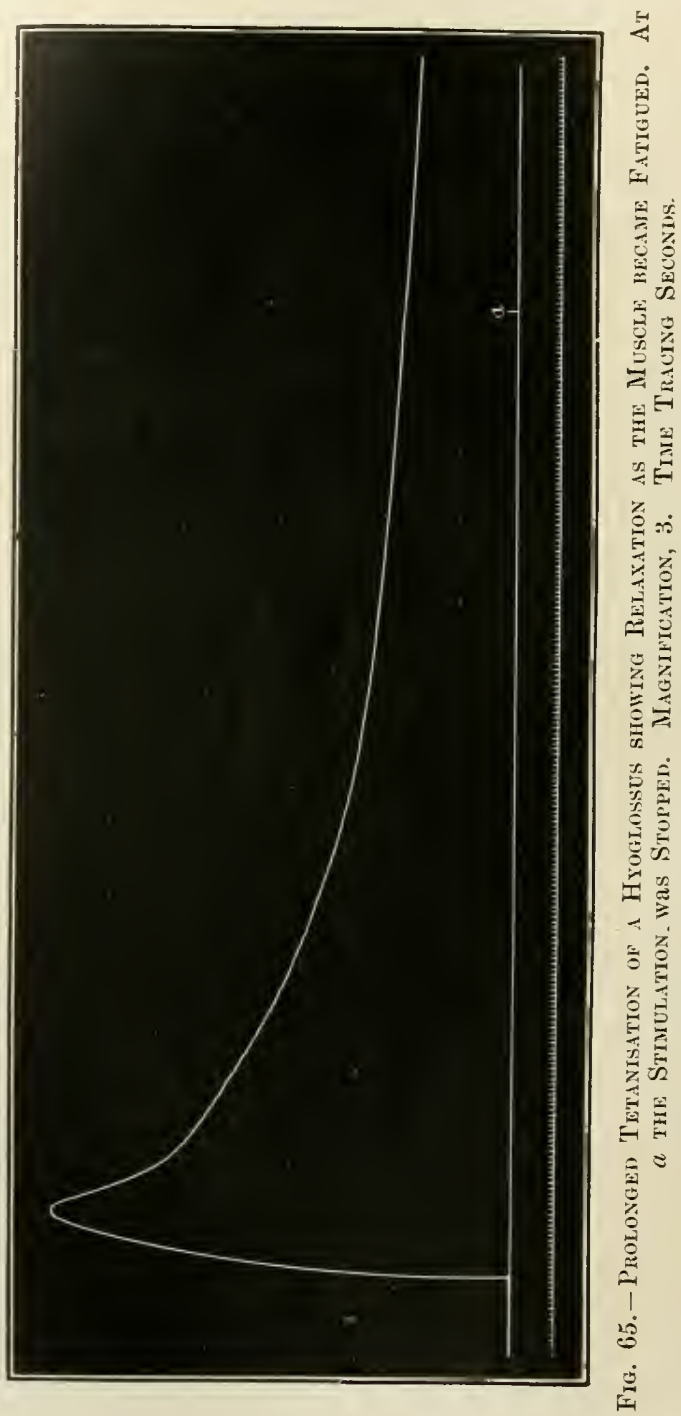

there is no sudden fall of the lever, but only a gradual fall at almost the same rate as during the latter part of the period of stimulation. The muscle has passed into a marked degree of contractur. 


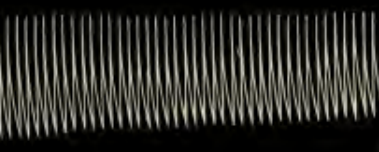

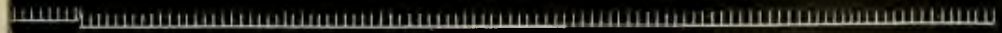

glossuion five-fold. Shows the alterations in the character of the twitch as the 


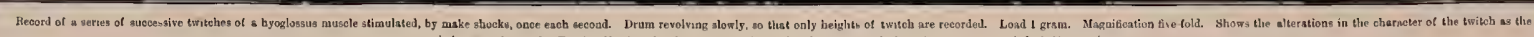

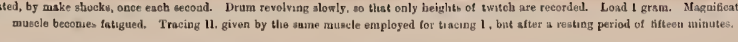


CHAPTER VI
THE THICKENING OF A MUSCLE ON CONTRACTION.

THE MUSCLE WAVE.

When a muscle shortens during a twitch it also thickens, and this thickening may be studied in the following experiment, whose object is to magnify and record the changes in a transverse diameter at some convenient spot.

Experiment 1.-Take a tracing of the thickening of a muscle during a contraction by means of the apparatus shown in fig. 66. Arrange the apparatus

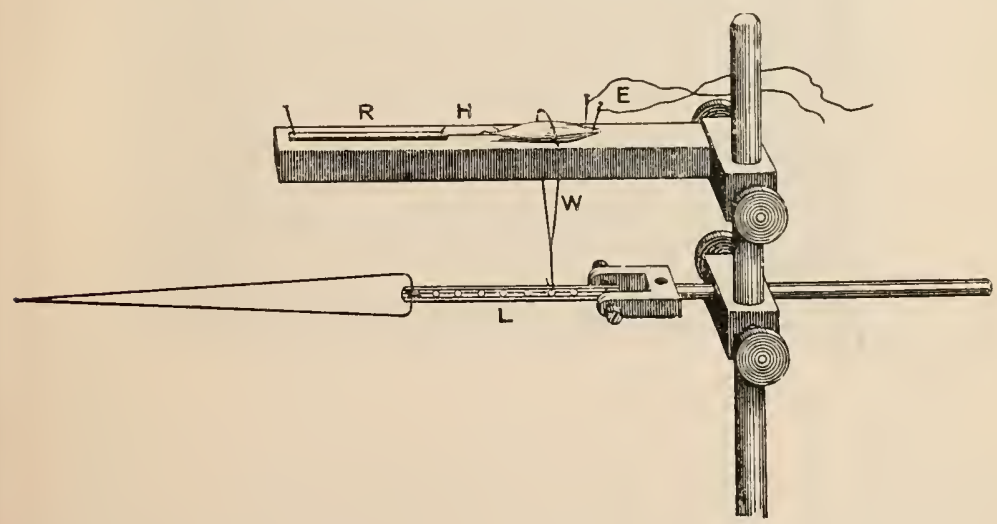

Fig. 66.--Method of Recording the Thickenisg of a Muscle as it Contracts.

in the same way as for a simple twitch, but use the recording apparatus shown in the figure. Make a gastrocnemius preparation from a curarised frog, and to its tendon attach a length of indiarubber, $\mathrm{R}$. The muscle is laid upon a narrow wooden support, $\mathbf{H}$, whose upper surface is covered with cork. The upper end is fixed to this base by two pins, to which two fine wires are soldered, thus forming a pair of electrodes. The end of the strip of rubber is pinned down to the cork base by a pin, so as to put the muscle slightly on the stretch. Resting on the muscle at a point near the electrodes is a light wire of aluminium, w, in the form of a hoop, the part touching the muscle being hammered out flat. This wire is connected below to a recording lever, $\mathrm{L}$.

1 The frog is previously curarised in order to prevent excitation of the muscle through its nerve (see pp. 76, 77). 
A tracing obtained by this arrangement is shown in curve II, fig. 67 , and for purposes of comparison a simple twitch taken immediately after, under precisely the same conditions, by attaching a thread from the tendon to a crank lever is reproduced in curve $\mathrm{I}$. The two curves show the following points of difference.

(i.) The latent period in the case of II is less than in I. The measurement given by the curve of thickening is just under $005 \mathrm{sec}$. This method is the one commonly employed for determining the latent period, the points to be especially observed being $(a)$ to make all

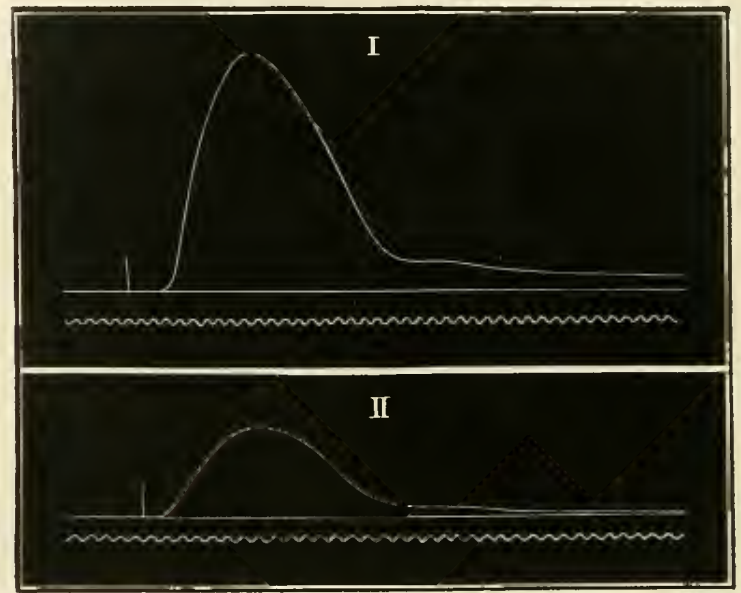

Fig. 67.-Corve I the Shortenixg, and Curve II the Thickenixg of a SejiMembranoses and Gracilis Preparation. Tine Tracing, 200 per Sec.

moving parts of the lightest possible character, and $(b)$ to stimulate the muscle exactly at the spot on which the recording lever rests.

(ii.) The amount of movement is very much less in the second tracing. This follows as a necessity when we remember that in the first instance the curve is a summation of the movements along the length of the whole muscle. The second is a summation of the different thickenings over one transverse section; and as the length is much greater than the thickness, that of itself explains the main difference. Further thickening is not proportional to the amount of shortening, which therefore further accentuates the difference.

(iii.) The total duration of the second is a little less than that of the first curve. This is because, in a contraction, the whole length of the muscle fibre does not contract simultaneously, but successively, whereas in the particular conditions of the experiment thickening 
occurs simultaneously throughout the section upon which the recording lever rests.

The Muscle Wave.-We have seen that when a muscle contracts, it passes through a series of phases, a latent period in which no change of form occurs followed by a period of shortening, and this again by one of relaxation; and the same holds true for each fibre and further for each constituent part of that fibre. If we, for the time being, limit our attention to what is happening in a single fibre, it is found that all parts do not pass through the different phases of their contraction synchronously, but that the contraction travels along the fibre in the form of a wave. A study of fig. 68 will make this clearer. A B is supposed to represent a very long muscle fibre, which has been stimulated at the point $A$, and as a result a wave of contraction, represented by the bulging of the fibre between $C$ and $D$, is travelling towards B. At a somewhat later instant than the one drawn, the condition of the fibre will be represented by the dotted lines, and the front of the wave of contraction will have reached the point $E$. D has then passed through about half of its phases, and at $\mathrm{c}$ the muscle has returned to its initial form. The wave of contraction will in this way

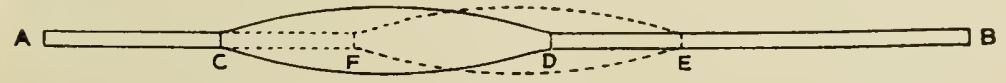

Fig. 68

travel on until it reaches and has passed over $\mathrm{B}$, when the whole fibre will come to rest. In the study of a wave of such a nature we can gain a full knowledge of it, if we can determine its following characteristics :-

(1) Its amplitude.

(2) The rate at which it travels.

(3) Its length.

Its amplitude and general course can be ascertained by recording the changes in diameter of a section of the fibre as the wave passes over it. The method by which this is done has been carried out in experiment 1 . Next by measuring the time interval between the first movement at $\mathbf{D}$ and the corresponding change at $\mathbf{E}$ we can determine its velocity after measuring the distance of $\mathbf{E}$ from $\mathrm{D}$. Thus suppose the distance $\mathrm{DE}$ to be $l$ centimetres, and the time occupied by the wave front in travelling from $\mathrm{D}$ to $\mathrm{E}$ to be $t$ seconds; then in one second the wave travels $\frac{l}{t} \mathrm{~cm}$., or in other words $\frac{l}{t}$ is the velocity per second in centimetres. Lastly, if we determine the time the wave takes to pass over any fixed point, we can determine its wave length 
$\lambda$, i.e. the length $\mathrm{C} \mathrm{D}$ of fig. 68 . For if this time be $t^{\prime}$ during that time a wave of velocity $\frac{l}{t}$ will travel $\frac{l}{t} \times t^{\prime}$ cms. But at the end of the measured interval $t^{\prime}$ the wave is just leaving the fixed point. Hence

$$
\lambda=\frac{l}{t} \times t^{\prime} \mathrm{cms} \text {. }
$$

Actual measurements show that this wave length is great as compared to the general length of a muscle fibre, so that a single fibre cannot present at any one instant all the phases representing a single wave of contraction. Referring to our diagram it is as if the part D E were the only piece available for observation, and we then had to study the wave of thickening as it travelled over that portion. Thus if we record the total changes at two positions on the fibre and the time relations of those changes, we can then determine all the characteristics of the wave. This we can do by the following method:-

Experiment 2.-Arrange the apparatus as for a simple twitch, fitting in a pair of pin electrodes. Curarise a frog and dissect out a gracilis and semimembranosus preparation. Arrange this as shown in fig. 69. The prepara-

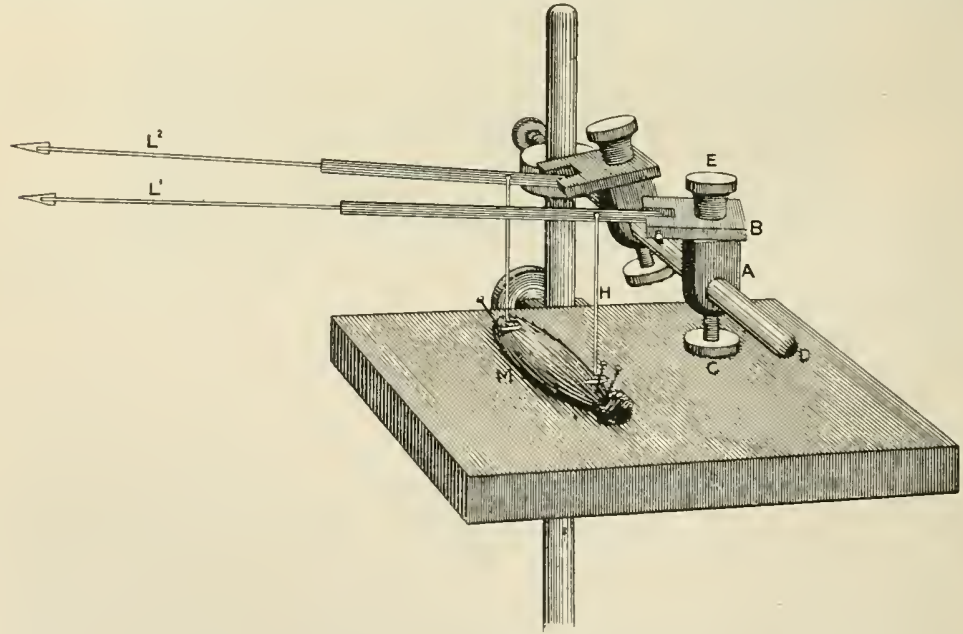

Fig. 69.-Apparates for Recording the Thichening of a Muscle at Two Pornts, for the purpose of studing the Muscle TVave.

tion $x$ is pinned down to a cork base, at one end a pair of pin electrodes being used. Two levers, $\mathrm{L}^{1}$ and $\mathrm{L}^{2}$, are then arranged as in the figure. Each lever consists of a light bar to which is jointed a light vertical bar terminating in a flattened foot to rest on the muscle. Each lever is held on a specially arranged block, $\mathrm{A}$, which slides along a bar, $\mathrm{D}$, and can be fixed in any position by a screw, c. The axis of the lever is carried on a plate, B, 
which can be rotated round the screw E, and by that screw clamped in any position to A. By movement along the horizontal rod $\mathrm{D}$ the levers can be approximated or separated to suit the length of the muscle. By rotation of the block $A$ around the rod $D$ the writing point of the lever can be raised or depressed, and finally by rotation of the plate $\mathrm{B}$ the writing point can be adjusted to the writing surface. By this apparatus we can record the changes in thickness of the muscle at two points separated by a distance which can be measured in millimetres. One lever is fixed near the pin electrodes, the other as far away as is convenient.

On recording a single twitch the tracing obtained is similar to that of fig. 70. In this experiment the vertical levers rested on the muscle at two points situated $16 \mathrm{~mm}$. from each other, and each therefore records the variations of thickness at those two points.

The electrodes were placed immediately under the point on which the upper lever rested. The time tracing is 200 per second. Hence

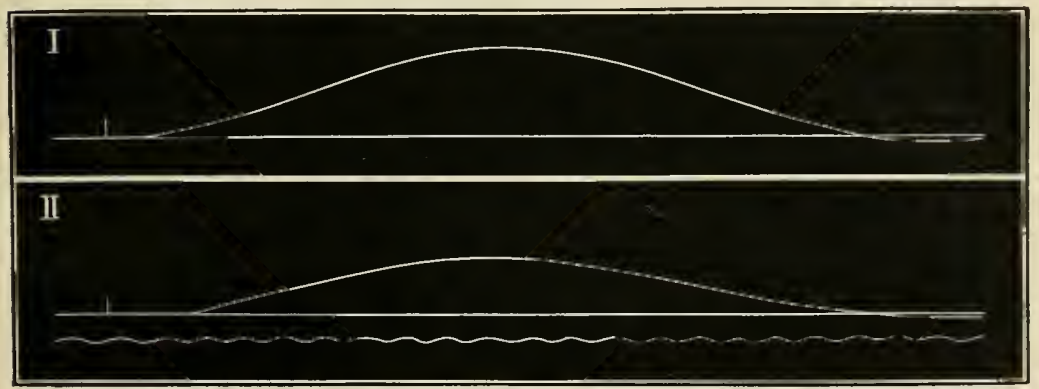

Fig. 70.- The Thickening of a Muscle; Curve I at 4 mi., axd Curve II at 20 am. From the spot Stimulated. Magnification, 8. Time Tracing, 200 PER Sec.

the measurements of the two latent periods are 0044 second and -0094 second respectively. The difference is 005 second. Hence the muscle wave travelled from the first lever to the second, a distance of $16 \mathrm{~mm}$., in 005 second; therefore its rate was 3.2 metres per second. The temperature of the room and hence of the muscle was, in this experiment, $18^{\circ} \mathrm{C}$.

The time occupied by the total variation of thickness is seen for both curves to be 085 second. Hence the length of the muscle wave was $\cdot 085 \times 3 \cdot 2$ metres $=27 \cdot 2 \mathrm{~cm}$. 


\section{CHAPTER VII}

INDEPENDENT MUSCULAR EXCITABILITY. EXCITATION OF MUSCLE

BY THE CONSTANT CURRENT. POLARISATION OF ELECTRODES

\section{INDEPENDENT MUSCULAR EXCITABILITY}

By this is meant the property a living muscle fibre possesses of responding by a contraction to a stimulus applied directly to it. To prove that the muscle substance is itself excitable, it is necessary to devise an experiment in which the stimulation of the muscle-fibre via its nerve is altogether avoided. The experiments commonly adopted for this purpose are the following:-

Experiment 1.-BERNARD'S CURARE EXPERIMENT.-Destroy the brain of a frog. Cut through the skin at the back of the left. thigh for about an inch, separate out a short length of the sciatic nerve, and pass a stout ligature under it. Bring the ends of the ligature to the front of the thigh and tie tightly, thus including the whole of the structures of the limb with the exception of the sciatic nerve. Now inject a few drops of a one per cent. solution of curare (Indian arrow-poison) into the dorsal lymph sac and allow the frog to remain for about half an hour. At the end of that time the animal has become quite paralysed with the exception of the left leg. Pinching the skin of the right leg produces no movement in that limb, but pinching the left leg leads to movements of the limb. Now dissect out both sciatic nerves right up to the vertebral column. Arrange a battery and coil for giving tetanising shocks and place the electrodes under the right sciatic nerve. Stimulation of the nerve in any part of its course has no effect upon the muscles which it supplies, but can produce reflex contraction of the leg muscles on the left side. Next place the electrodes under the left sciatic. Stimulation of this nerve causes contractions of the leg and foot muscles of the same side wherever the electrodes are placed, whether close to the knee where it has not been exposed to the action of the poison in the blood, or at that part near the vertebral column where the poison has been able to reach it. Finally apply the electrodes directly to any of the muscles of the right leg. They contract on stimulation. 
The action of the curare has been to destroy the physiological continuity of muscle with its nerve fibre. The poison injected is rapidly absorbed into the blood, and so carried to all parts of the body with the exception of the left leg below the ligature, where the vessels have been occluded. The upper part of the left sciatic has been exposed to the poison, but is still excitable, showing that the action of the poison is not on nerve-fibre; and as the muscles in any part of the body contract when directly excited, it follows that they are not the parts affected by the poison. The portion paralysed must therefore be situated at the connection of the nerve to the muscle, i.e. the motor end plate or the small terminal piece of nerve which is unprotected by a medullary sheath. Just at this point, moreover, a very complex net of blood vessels is found, so that the part would be freely exposed to any poison present in the blood.

From the same preparation we may also show another fact which further tends to the same conclusion.

Experiment 2.-Using single induced break shocks determine for the left nerve the strength of stimulus necessary to just cause a twitch of the gastrocnemius, and secondly that which gives a maximum twitch. Having done this, apply the electrodes directly to the paralysed gastrocnemins, the right, and determine the two corresponding positions for the muscle.

It will be found that in the latter half of the experiment the stimulus must be greater for both cases. This tends to confirm the previous result, viz. that it is muscle fibre which is being stimulated, and not nerve fibre, and in that case shows that MUSCLE IS LESS EXCITABLE THAN NERVE,

Experiment 3.-Kühne's Sartorius Experiment.-Carefully dissect out a sartorius, and to the tendon which attaches it to the tibia tie a fine thread. The upper end of the muscle may be freed from its attachment to the symphysis. Suspend the muscle with its upper end hanging downwards, and bring up under it a drop of glycerine in a watch glass until the end of the muscle just touches the glycerine. No contraction results. Cut off the end which has touched the glycerine, and note that the muscle contracts under the mechanical excitation. Again touch the cut surface with glycerine. If only about $1 \mathrm{~mm}$. of the end has been cut off there is again no contraction. Cut off a fresh millimetre of muscle and repeat as before. It will be found that when about 3 to $4 \mathrm{~mm}$. of the upper (pelvic) end has been cut away, on contact of the freshly exposed end with the glycerine, the muscle shows irregular twitchings, and is at last thrown into a state of incon. plete tetanus. This experiment should in the next place be supplemented by showing that if a gastrocnemius nerve muscle preparation be mule, and the cut end of the nerve dipped into glycerine, the gastrocnemius is thrown into a similar series of irregular twitchings. Nerve fibre is therefore excitable to glycerine.

The explanation of the preceding experiment becomes clear by the 
light of a knowledge of the distribution of the nerve fibres in the sartorius. Kühne showed that the upper 4 to $5 \mathrm{~mm}$. of the sartorius contained no nerve fibres, nor could nerve-endings of any kind be traced in this part. The same holds good for the lower 2 to $3 \mathrm{~mm}$. Hence in the first part of the experiment only muscle fibre was being exposed to the glycerine; and as the muscle did not contract, it follows that muscle fibre is not excitable to glycerine. As soon, however, as the lower part had been cut away, some of the nerve fibres became exposed, and as they are excitable to glycerine the muscle was thrown into a series of irregular twitchings.

Experiment 4.-Upon the sartorius of the opposite limb perform an exactly similar experiment, but use a salt solution ( 0.65 per cent.) 'sontaining a drop of ammonia solution. The muscle will be found to be extremely sensitive to this, even the rapour of $\mathrm{NH}_{3}$ from it being quite sufficient to throw it into tetanus. Nerve, on the other hand, is not excited by it. Prove this by dipping the freshly cut end of the sciatic nerve of a gastrocnemius preparation into the solution, taking care that the muscle is thoroughly protected from the vapour by folds of blotting-paper moistened with normal saline solution. It is best, too, to hold the watch-glass of ammonia solution above the level of the muscle, and to lift up the nerve by a fine glass rod bent into the form of a hook, and thus dip the cut end of the nerve into the solution. No contraction of the gastrocnemius results, but the nerve is not unaffected, for it will be found that the part which has been exposed to the fluid, if tested by electrical stimnli, has been killed.

Experiment 5.-Kü̈nne's Curare Experiment.-Pin down two strips of copper foil upon a flat plate of cork with their ends about $4 \mathrm{~cm}$. apart, and join them by a strip of blotting-paper moistened with 0.65 per cent. $\mathrm{NaCl}$ solution. Connect the copper strips to the secondary coil of an inductorium arranged for tetanising. Prepare a sartorius and cut it transversely into five pieces of equal length, and arrange these in series upon the strip of moist blotting-paper. Starting with the secondary coil at some distance from the primary, send tetanising shocks through the preparation, gradually increasing the strength of the stimulation until at last one is found which causes the three middle pieces of the sartorius to contract while the upper and lower ends remain quiescent. Increase the strength of the stimulation still further, when the two terminal pieces are also thrown into contraction. If a curarised sartorius be employed all five pieces go into tetanus at once, viz. when the stronger stimulus which was required to tetanise the two terminal pieces in the first experiment is reached.

The difference in behaviour of the five pieces is due to the fact that the two ends contain no nerve terminals, while the three centre-pieces do, and as was seen from a previous experiment (experiment 2) muscle is less excitable than nerve.

In addition to these experiments other facts are known tending to show the inherent excitability of muscle. Nerve, for instance, is not excited by stimuli which are arranged at right angles to the direction of the fibres, it being necessary that the stimulus or part of it should pass in the same direction as that of the fibres. Muscle, on 
the contrary, is quite as excitable to stimuli in a direction transverse to the muscle fibres as to one parallel to them. Nerve again is especially sensitive to currents of very short duration, whereas muscle will not respond unless the duration be sufficiently prolonged. A curarised muscle is much less sensitive to shocks of short duration than a non-curarised one.

In a further direction Kühne showed that chemical stimuli which were particularly irritant to muscle (such as $\mathrm{NH}_{3}$ or very weak $\mathrm{HCl}$ ) were equally effective to both curarised and non-curarised muscles. If a strong constant current be sent through a nerve, it is found that the excitability of the nerve in the part immediately surrounding the anode is very greatly diminished. Kühne utilised this to lower the excitability of the nerve fibres in a sartorius by throwing a constant current into its nerve, placing the anode close to the muscle. A muscle thus treated was found to be just as excitable to ammonia or weak hydrochloric acid, whilst those forms of stimuli, such as glycerine, which act on nerve only, are now without effect, or only produce one when the excitation becomes excessive.

If the nerve supplying a muscle be cut and allowed to degenerate for some days, the response of the muscle to electrical stimuli becomes considerably modified; while the intra-muscular nerve endings are intact the muscle responds more readily to induced shocks than to the constant current, whereas when these terminals have degenerated the reverse is found to be the case. This change of condition is explicable on the fact already tested in experiment 2, which shows that muscle is much less excitable to currents of short duration than nerve.

\section{EXCITATION OF MUSCLE BY A CONSTANT CURRENT}

In our experiments up to this point, we have as a rule employed an induced shock whenever we wished to stimulate a muscle or its nerve, but we have also seen that a muscle is excitable to thermal, mechanical, and chemical stimuli as well as to electrical. We have now to consider the response of muscle to electrical stimuli other than induced currents. We found that muscle was less excitable to induced currents than nerve, and this is found to be due to the very short duration of these currents.

If a constant current of sufficient strength be sent through a muscle a contraction occurs at the instant of make of the current and again at the instant of break, but no effect is as a rule produced during the passage of the current. These two contractions are different, not only 
in amount, but in that they start from different points. Calling the electrode at which the current enters the muscle the anode, and that at which it leaves, the kathode, it is found that on make of the current the contraction starts from the kathode and thence spreads over the muscle, but that on break the contraction starts from the anode. This very important point in the response of muscle to an electrical current can be shown by the following experiments :-

Experiment 6.-Dissect out a sartorius and fix it to record its movements as shown in fig. 71. The muscle is lightly clipped between two pieces of cork $\mathrm{C}$ and $\mathrm{D}$ at a point near its tibial end. A fine thread is tied romnd the tendon at that end and attached to a crank lever, L. Two pins are passed through the corks $\mathrm{C} \mathrm{D}$, and by these the muscle is fixed to the myo. graph plate. The remaining longer piece of the muscle is connected to two electrodes (unpolarisable, see p. 83) A and B, which are connected to a constant current throngh a Pohl's commitator with cross wires, so that the direction of the current may be reversed. The muscle is clamped so tightly by the corks that it prevents any movement at $\mathrm{A}$ or $\mathrm{B}$ pulling on the piece of sartorius $\mathrm{s}$ to the left of the cork clamp, and so moving the lever. It is not, however, so tightly clamped as to prevent a wave of contraction passing across from the piece $\mathrm{s}^{1}$ to the piece $\mathrm{s}$. If now a contraction start at one instant from $\mathrm{A}$, and passes as a wave along the muscle to $\mathrm{s}$, a longer interval will elapse before $\mathrm{s}$ begins to contract and raises the lever than if the contraction started at $\mathrm{B}$ and had only a short piece of muscle to travel along

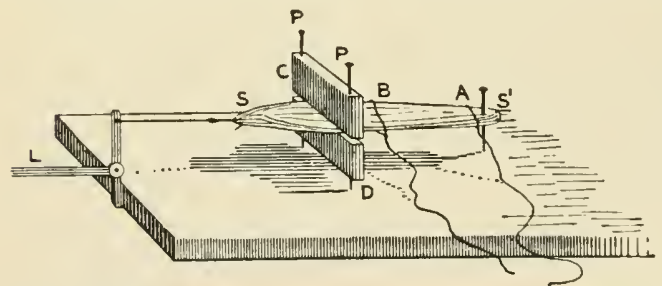

Fig. 71.- Method of studying Polar Excitation of a Muscle. before it reached s. The experiment now consists in measturing the latent periods of four curves: two, one at make, the other at break of a constant current when the current passes from $\mathrm{A}$ to $\mathrm{B}$, i.e. when the anode is at $A$, and two when the current travels from $\mathrm{B}$ to A, i.e. when the anode is at B. To record the in. stants of opening and closing the current a signal inclinded in the primary circuit is arranged to record its movements directly beneath the myograph lever. This does not give us an absolutely accurate measurement of the latent period because there is a latency in the signal; but as this is the same in all four cases this does not matter, for we only require to measure differences in the latent period.

It will be found that make of the ascending current (anode. at $\mathrm{B}$ ) and break of the descending current (anode at $\mathrm{A}$ ) have practically the same latent period, and both are greater than those on break of the ascending current or make of the descending current, which in their turn are practically equal in value.

Hence it is argued that the contraction on make of an ascending 
current starts from $\mathrm{A}$, and of a descending current from $\mathrm{B}$, in both cases the kathode; and, on the other hand, that the contraction on break starts from $\mathrm{B}$ with an ascending, and from $\mathrm{A}$ with a descending, current, these two points being the anodes in the two cases. The differences are more clearly seen if the muscle be fatigued.

Experiment 7.-Engelmann's experiment. Curarise a frog, dissect ont its sartorius, and suspend it by its pelvic end. Arrange two electrodes to send a current transversely across this end. On closing the current the free end moves towards the kathodic side of the muscle, on opening towarts the anodic.

Experiment 8.--Prepare a sartorius from a curarised frog that has been previously kept at a low temperature for some hours. Place it on a pair of unpolarisable electrodes. On closing the current the muscle passes into tetanus, and if the muscle be carefully observed, it will be seen that the only part in persistent contraction is that around the kathode. On opening, the muscle also commonly passes into tetanus, but in this case the contraction is limited to the region of the anode. This experiment is all the more striking if Biedermann's plan, of striping the sartorius transversely with black lines made by a bristle dipped in china ink, be adopted. The region in contraction is then clearly marked by the approximation of the black lines. The non-contracted part is seen to be stretched ont, either by an actnal stretching due to the contracted part pulling on it, or due to an active relaxation in that region. The latter is probably the main cause, as is well exemplified by the following experiment.

Experiment 9.-A frog is pithed and its heart exposed. One electrode is now placed on any part of the body, and the point of the other, which should be a fine wire, is applied to the heart. If the electrode on the heart be the anode it will be seen that at each contraction the part around the point tonched does not pale like the rest of the heart, i.e. that region does not contract but is inhibited. Conversely, if it be the kathode, it is seen that that spot remains pale during relaxation of the heart, which means that those fibres immediately affected do not relax properly. This latter point is not so easy to make out as the former.

Polarisation of Electrodes. - If a pair of clean platinum wires be immersed in water, and a current sent through them for a time, it is found that both of the platinum terminals become covered with bubbles of gas. That one in connection with the negative pole of the battery is covered with hydrogen, and the other with oxygen. If now the battery be removed and the two electrodes connected to the two terminals of a galvanometer, it is found that a current is shown by the galvanometer, which has a direction, in the galvanometer circuit, from the electrode covered with oxygen to that covered with hydrogen. It is, in other words, in the reverse direction to that of the initially employed current. The production of this state at the electrodes is spoken of as polarisation of clectrodes. The same usually occurs, though to different degrees, if solutions of salts be tested instead of distilled water, and no matter what metal the electrodes are made of. Regnault discovered, however, that if the electrodes were made of 
pure zinc, and the solution in which they were immersed was a strong solution of zine sulphate, that no polarisation occurred; and the same was found to be the case with less purified zinc if its surfaces were thoroughly amalgamated.

If instead of a solution a piece of fresh animal tissue connects a pair of wire electrodes the same polarisation occurs. Chemical changes are set up where the wires touch the tissue which can act in the reverse manner, and set up a small current if the battery be removed and the electrodes connected by a conductor. This acts as a source of fallacy in many experiments, and becomes of great importance where we are dealing with a very excitable tissue, such as nerve. The existence of this polarisation current may be proved, as in the previous example, by sending it through a galvanometer ; but we are also able to show it by its effect in exciting a nerve, as in the following arrangement :-

Experiment 10.--Arrange the apparatus as shown in fig. 72 , open the key $\mathbf{K}_{2}$ and close the key $k_{1}$. The current is thus sent through the nerve by the electrodes $\mathrm{E}$, which it will polarise. Note that there is no contraction during

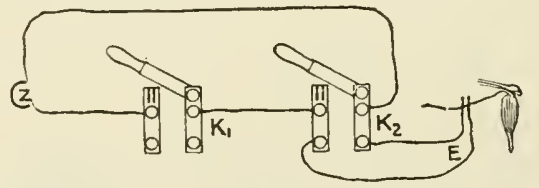

Fig. 72.-Method of Arranging the Apparates to Show Polarisation of Electiodes.

the time the current is passing. In abont a minute open $k_{1}$ and then rapidly close and open $\kappa_{2}$, when contractions will occur which are due to the closure and opening of the small current set up by the polarised electrodes. The contractions rapidly diminish in amount as the nerve becomes depolarised.

This experiment illustrates the necessity of avoiding this polarisation, if it be possible, when we are experimenting upon nerves or other excitable tissues, and Du Bois-Reymond utilised Regnault's discovery for making electrodes which would not show this defect. In his original form (fig. 73,1 ) each electrode consisted of a zinc trough on an insulating vulcanite base, the outer surface being thoroughly varnished and the inner well amalgamated. Into this a thick pad of filter paper thoroughly soaked in a saturated solution of zinc sulphate is fitted, and the part of the pad lying in the trough is then covered with the saturated zinc sulphate solution. If, now, the piece of tissue be placed between two such pads the zinc salt rapidly produces corrosive effects, and the tissue is rapidly destroyed. To prevent this, little masses of china clay worked up into a stiff paste with normal saline solution are used, upon which to rest the tissue and 
connect it with the electrodes. These do not cause any polarisation, and at the same time are fairly good conductors. They are spoken of as clay-guards. In many cases this form of electrode is too large, and it becomes impossible to bring them into contact with any two

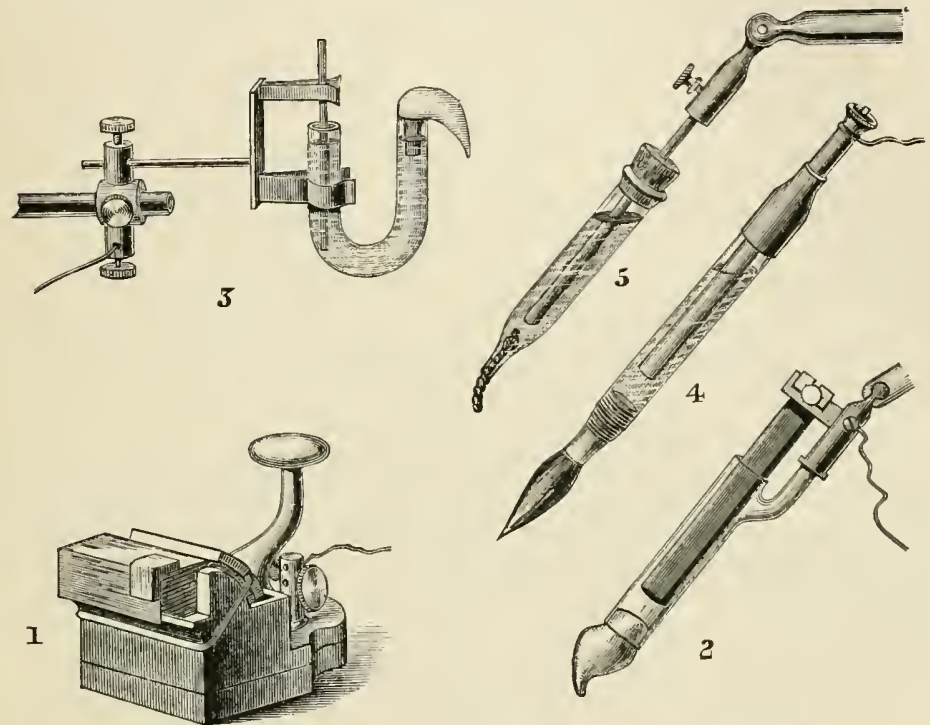

Fig. 73.-Several Models of Unpolarisable Electrodes

1 and 2, Du Bois-Reymond's; 3, Burdon-Sanderson's ; 4, vou Fleisehl's ; 5, d'Arsonval's.

In $1,2,3$, and 4 the component parts are zine, zine sulphate, and saline clay; in 5 a silver rou coated with fused silver chlorile dipping in normal saline contained in the tube from which a thread projects. (Waller.)

desired points of the tissue to be experimented upon; and to overcome this many forms of electrode have been employed by different workers. Some of these are shown in the accompanying figure (73).

Make a pair of unpolarisable electrodes in the following way. Take a piece of glass tubing of fairly thick walls and with an internal diameter of about $\frac{1}{4}$ inch. Rotate this with its centre in a blowpipe flame without drawing it out until the central part becomes of a less diameter; then draw it out slightly and allow to cool. Cut it through the centre of the constricted part and round off these ends in a flame. The bore at this end should now be about $\frac{1}{16}$ inch. Cut the tubes so that they are $2 \frac{1}{4}$ inches long and round off the upper ends in a Hame. This glass tube is shown at G, fig. 74 . Take a cork and bore half through it with a cork-borer of such size that the upper end of the glass tube is held firmly

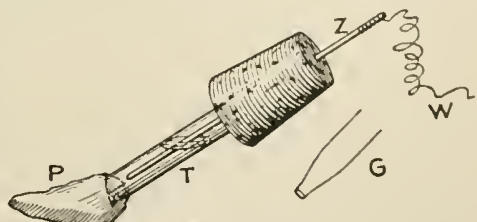

Fig. 74. - Simpie Foni of UnpolarisABLE ELECTRODES.

in the hole bored. With a fine bradawl pierce the cork from the upper end, and this will remove the lower bored piece of cork if that has not already comeaway 
inside the borer. Solder an insulated wire to one end of a straight piece of pure zinc wire of about three inches length, and then thoroughly amalgamate the zine wire by dipping it into the amalgamating fluid and rubbing it on a clean duster. Now pass the zinewire through the hole in the cork made by the bradawl until it projects well on the lower side. Next take some powdered kaolin and make it into a stiff clay with normal saline, and force a little of this up the constricted end of the glass tube to act as a plug to close that orifice. Then fill the tube with saturated zine sulphate solution, and fit it into the large hole in the cork, so that the zine wire dips into the solntion. All that is now required to complete the electrode is to fix to the lower end a plug of china clay whose apex may be moulded to any desired shape (see fig. 74). In many cases it will be found convenient to fix in the centre of this chinaclay guard a coarse thread soaked in normal saline, which can then act as the terminal part of the electrode. These electrodes can be kept, and all that they will require at a future time is a fresh guard of clay, when they will at once be ready for use.

Experiment 11.-Arrange the apparatus exactly as in experiment 10 , fig. 72, but employ these electrodes in the place of the wire ones of that experiment. The nerve does not now become polarised.

Great as is the importance of using unpolarisable electrodes when a current is to be sent through a nerve, their use is of still greater necessity when we require to examine the currents produced by a tissue, and for this purpose are making use of a sensitive galvanometer. 
In examining the functions of a nerve we have two main methods which we may employ, section and stimulation.

By section we can observe the loss of function resulting from the loss of impulses normally passing along it.

By stimulation we can observe the converse effect, i.e. the production of some functional activity, such as a muscular movement, secretion of a gland, \&c., when impulses are sent along the nerve.

The two methods of experimentation illustrate the two great physiological attributes of a nerve, viz. conductivity and excitability. Section teaches us the function of a nerve by observation of the results of interrupting its conductivity at some spot. Stimulation makes use of its property of excitability to give us knowledge of the results of impulses travelling along the particular nerve.

We have already made use of a nerve's excitability in our experiments upon muscle, and in our study of the variations of function of a nerve under different experimental conditions motor nerves are generally employed, because the muscular response enables us to readily determine small changes in the nerve's activity.

As conductors nerves carry impulses either to or from the central nervous system, i.e. they are afferent or efferent in function. At their entrance into the cord, the spinal nerves divide into two roots, anterior and posterior, the former carrying only efferent, the latter only afferent impulses. Show this upon the frog by the following experiment :-

Experiment 1.-Decapitate a frog and cut away the upper third of the vertebral column. With a fine pointed pair of scissors eut away the laminæ of the remaining vertebræ, taking great care to avoid injuring the spinal cord. This brings to view the cord in its lower part with the large roots of the nerves which form the sciatic plexus. To see the anterior roots, the posterior, which cover them up, must be displaced. (i.) Lift up the largest posterior root with a seeker and pass a silk thread under it. Tie this close to the cord and cut through the root between the ligature and the cord. Note that on tying and on section irregular movements of the limbs are caused which 
vary, however, according to the degree of stimulation. Place the peripheral end upon a fine pair of electrodes and stimulate. There is no muscular contraction.

(ii.) Place a second ligature round another posterior root, this time tying as far from the cord as possible, and cut through the nerve peripherally to the ligature. Stimulate the central end, i.e. the part still attached to the cord. The limbs are thrown into convulsions, more or less marked and extensive according to the strength of the stimulation. These two stimulations prove that the posterior roots contain no efferent, but do contain afferent fibres.

(iii.) Cut through all the posterior roots of that side. Any mechanical stimulation to the skin no longer produces movements of the legs, though stimulation of the skin of the opposite leg will produce movements in both.

(iv.) The section of the posterior roots brings the anterior into view. By placing ligatures round two of these and tying $(a)$ near the cord and $(b)$ near the junction with the posterior root show that stimulation of the peripheral end leads to contraction of muscles, but stimulation of the central end produces no effect. The anterior root therefore contains efferent but no afferent fibres.

(v.) Cut through all the anterior roots, and then show that no movement of that leg can now be produced by stimulation of the skin of the leg of the opposite side.

STIMULI which affect a nerve may be classified as follows :-

\section{Thermal.}

Experiment 2.-Make a nerve muscle preparation and touch the end of the nerve with a copper wire which has been heated for a few moments in a Bunsen flame. The muscle contracts. That heat should act as a stimulus it is necessary that the temperature should be high. If heat be gradually applied it will kill without stimulating.

2. Mechanical.

Expcriment 3.--To produce mechanical stimulation cut through the nerve used in the previous experiment just below the point to which the hot wire was applied. The muscle contracts, thus showing that the mechanical process of cutting has stimulated the nerve. In the next place, pinch the upper end of the nerve and show that this also acts mechanically as a stimulus. Show also that the nerve can be stimulated by giving it a sharp tap with the edge of the handle of a scalpel, thus pinching it between the scalpel and the solid support upon which it rests.

3. Chemical.

Experiment 4.-Take a nerve-muscle preparation and lift up the nerve on a glass rod bent as a hook, so that the cut end of the nerve hangs down. Touch the cut surface of the nerve with a drop of a . 8 per cent. $\mathrm{KOH}$ solution in normal saline, held in a watch-glass. With each contact the muscle contracts, and, if the nerve be immersed a little time, passes into an irregular tetanus. Cut away the piece 
of nerve that has been in contact with the fluid. The muscle relaxes.

Experiment 5.-Next immerse the cut end in a drop of methylated spirit. This, too, causes contraction.

Experiment 6.--Repeat, using a solution of 15 per cent. NaCl. The same result is obtained.

There are very many substances which excite a nerve chemically. Of these we may mention glycerine in strong solutions, solution of lactic acid, bile salts, or mineral acids if not too dilute. Substances which kill but do not excite are basic or neutral lead acetate, chromic acid, copper sulphate, ammonia, \&c.

\section{Electrical.}

Stimulation by an electrical current has already been frequently employed in the experiments upon muscle, when we used indirect stimulation.

\section{THE EFFECTS OF THE CONSTANT CURRENT UPON NERVE}

Employing a current of medium strength, e.g. one Daniell, the usual result is that a twitch occurs at make and break of the current, but that during the passage of the current the muscle remains quiescent, i.e. the nerve is not stimulated. But this is not universally true, for if the nerve be very irritable, there may be produced a tetanic contraction the whole time the current is passing. This can always be produced if the frog from which the nerve muscle preparation is taken has been kept for a day or two at a low temperature, between $0^{\circ} \mathrm{C}$. and $10^{\circ} \mathrm{C}$. A tetanus at make or break is especially liable to occur at the make of a strong descending current, or the break of a strong ascending current, where by an ascending current is meant one in which the direction of the current in the nerve is from the muscle towards the spinal cord, and by a descending the reverse. This condition is spoken of as Ritter's tetanus. A Ritter's tetanus at break of an ascending current may be stopped by closing the current in the same direction, or may be increased by sending in a current in the reverse (descending) direction. Therefore, as a general rule, the nerve is not stimulated during the whole of the time of passage of a constant current, but only when the strength of the current is suddenly varied. It follows that when we were using induced shocks the excitation was in reality double, but the two stimuli followed one another at such a short interval that the effect on the nerve was the same as a single stimulation. Though the nerve is not 
being stimulated the whole of the time the current is passing, still we can show that its condition is altered. This altered state, due to a constant current, is spoken of as electrotonus, and its effect is expressed as an alteration in the two physiological properties of a nerve - its conductivity and its excitability. The alteration is different in the parts of the nerve in the neighbourhood of the two points at which the current is sent in and taken out of the nerve, i.e. the anode and kathode respectively. The condition of the nerve in the neighbourhood of the anode is termed anelectrotonus, that in the neighbourhood of the kathode katelectrotonus.

\section{Changes in excitability.}

Experiment 7.-Test the changes in excitability in the following manner. Arrange the primary and secondary coils for giving single induced shocks (left half of fig. 75), using a pair of fine wire electrodes, $\mathbf{E}_{1}$. Fit up a 'polarising' circuit with two batteries, a mercury key, $\mathrm{K}_{2}$, and a mercury commutator with eross wires, $\mathrm{K}_{4}$. To the commutator attach a pair of unpolarisable electrodes, $\mathbf{E}_{2}$ (right half of fig. 75). Dissect out a nerve muscle pre-

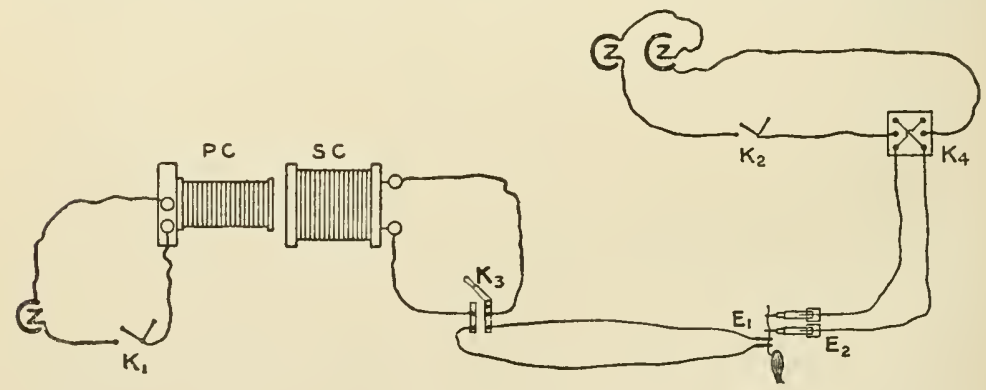

Fig. 75. - Plax of Apparatus for Situding the Changes of Excitability of Electrotonus.

paration, taking the whole length of the nerve, and being careful not to injure it during the dissection. Fix the gastrocnemius in the crank myograph and place the nerve across the nnpolarisable electrodes. Place the wire electrodes under the nerve at a point just below the unpolarisable electrodes nearest the muscle. With the key $\mathrm{K}_{2}$ still open, move the secondary coil to such a position that the muscle gives a small twitch on break of the primary circuit. Record the height of this twiteh on a stationary drum. Nove the drum a little by hand and close the polarising circuit. Note that the muscle gives a twitch. Again stimulate the nerve with a break shock and record the twitch. It will be found that either the twitch is of less. height or even absent, or that it is of greater height. Now examine the direction of the constant current through the nerve. If the twitch has been prevented or diminished, it will be found that the current ascends along the nerve from muscle towards vertebral end, and therefore the part of the nerve stimulated is in anelectrotonus. If, on the other hand, the twitch has been increased in height, it will be found that the electrode nearest the nerve is the kathode. After obtaining one of these two results, reverse the direction of the polarising current by the commutator $K_{4}$, when the second result will be obtained 
Experiment 8.- Repeat the preceding experiment, but tetanise the nerve at the spot to be tested instead of giving it a single stimulus. The shocks used should be just above minimal stimuli. Record the results obtained on a slowly moving drum. It will be found that if the spot stimulated be in anelectrotonus that the contraction on tetanisation is either abolished or diminished; if, on the other hand, it be in katelectrotonus, the contraction is increased.

These experiments show that a piece of nerve in anelectrotonus is less excitable than normally, for it does not give so large a contraction, and, on the other hand, the state of katelectrotonus is characterised by an increase of excitability. These same results have also been confirmed by other modes of stimulation, both mechanical and chemical. The latter can very easily be shown by the following experiment :-

Experiment 9.-Instead of using the secondary coil for stimulating, paint the nerve with a 10 per cent. solution of $\mathrm{NaCl}$ at a spot between the lower electrode and the muscle. In a short time the muscle is set twitching by the stimulus. Arrange the polarising circuit to give an ascending current and close the key $\mathrm{k}_{3}$. The twitching ceases. Reverse the key, so that the kathode is now nearest the muscle. The twitching becomes more marked. Record these results on a slowly moving drum.

By varying the course of experiments of this kind differences have been made out in regard to the degree of change of excitability and the time it lasts. The katelectrotonic increase of excitability reaches its maximum height directly after the closure of the current, and then gradually decreases whilst the anelectrotonic decrease develops and extends much more slowly, its maximum being reached some time after closure. The amount of change of excitability, as measured by the amount of contraction, produced by a stimulus of fixed strength is found to vary considerably with the strength of the polarising current. As the excitability is increased around the kathode and decreased around the anode, it follows that there must be one spot between anode and kathode at which excitability remains unchanged. This is called the indifferent point. The changes of excitability are conveniently represented diagrammatically, as in fig. 76 . In this figure the abscissa line $g i$ is taken to represent the nerve, and the polarising current is supposed to enter at $\mathrm{A}$ and leave at $\mathrm{k}$. An increase of excitability is represented by an ordinate above the abscissa, a decrease by one below. With a weak current the changes in excitability are represented by the curve $a n b r c$, which means that at the point $m$ of the nerve the excitability is decreased to an amount represented by the vertical $m n$. At the point $p$ it is increased by an amount represented by $p r$. Similarly for all other points between $a$ and $c$. The indifferent point is at $b$, which is seen to be nearer the anode $\mathrm{A}$ than the kathode $\mathrm{k}$. The changes in excitability 
are also seen not to extend far beyond the anode and kathode. For a stronger polarising current the changes are represented by the curve $d e f$. The indifferent point has moved towards the kathode; the changes are relatively greater and extend over a longer piece of nerve, $d f$. With a still stronger current the changes are represented by the curve $g h i$ in which the indifferent point is still nearer the kathode,

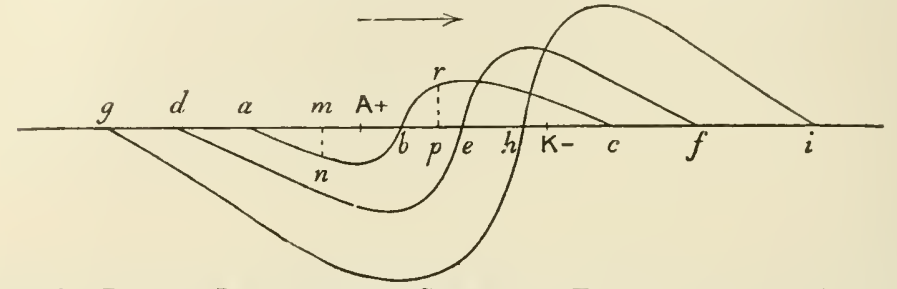

Fig. 76.-Diagrau Indicating the Chaxges of Excitability of a Nerve in ELEctrotonus.

and there is a further increase in intensity of effect and in extent of nerve involved.

Immediately after opening the current these changes are for a short time reversed, the excitability in that part of the nerve which was in anelectrotonus is increased, in that part previously in katelectrotonus decreased. These reversed effects gradually disappear.

\section{Changes in conductivity.}

Experiment 10.-Arrange the apparatus as in fig. 75, but place the polarising electrodes near the muscle and the exciting electrodes at the npper end of the nerve. Tetanise the nerve, and while the contraction still continues close the polarising current. The tetanus ceases. Repeat, but instead of tetanising apply a crystal of salt or a drop of strong salt solution to the cut end of the nerve. The twitchings carsed by the salt can be stopped by throwing in the polarising current. The same effect is produced whether the polarising current be ascending or descending. The polarising current has acted as a block, i.e. the nerve at that part has had its conductivity depressed.

The change of conductivity has also been studied by observation of the rate of transmission of a nerve impulse along a piece of nerve in anelectrotonus or in katelectrotonus, and further by observations of the changes of strength of the negative variation ${ }^{1}$ as it travels along the nerve. Such experiments showed that for all currents, except the weakest, conductivity was depressed in both the katelectrotonic and anelectrotonic states.

\section{POLAR EXCITATION OF NERVE}

When stimulating a muscle by the constant current we found that the stimulation at make started from the kathode and at

${ }^{3}$ For the explanation of the term negative variation see p. 146. 
break from the anode, and the same is found to be the case with nerve. As in the analogous experiment upon muscle, the starting points of the two excitations have been determined by time measurements of the two latent periods of the make and break contractions. The electrodes are separated as widely as possible; then if the stimulus start from the upper electrode, the latent period should be longer than if it started from the lower. Experimenting in this way it is found that if the current be ascending, the latent period of the contraction on make is longer than that of the contraction on break. From this we infer that the contraction on make starts from the upper electrode, i.e. the kathode, and that on break from the lower, i.e. the anode. A converse result is found for a descending current.

We may also state the fact of polar excitation in the following way. The production of katelectrotonus and the disappearance of anelectrotonus stimulate a nerve. Or, again, the passage of a nerve from a condition of lesser to one of greater excitability stimulates it.

In examining the effect of opening and closing a constant current through a nerve the results obtained are found to vary with the strength and direction of the current, and to study these variations we must possess some means of conveniently varying the strength of the constant current. This is afforded by either of the two following pieces of apparatus :-

The monochord is an application of the principle of the deriving circuit and is employed for varying the strength of current to be sent through a nerve or other tissue. Fig. 77 illustrates the method of using a monochord. A eurrent is sent through a stretched wire, $\mathrm{A}$ B, which must not be of too low resistance. The two electrodes are connected, one to one terminal, $A$, of the wire, the other to a movable contact, s. Supposing now the electrodes $\mathrm{E}$ to be bridged by a nerve, when the key $\mathrm{K}$ is closed the current on reaching $\mathrm{A}$ divides, part passing along $\mathrm{A} \mathrm{s}$, the other through the nerve. The amount of current passing through the nerve is inversely proportional to the resistance of that part of the circuit, and directly proportional to the potential difference

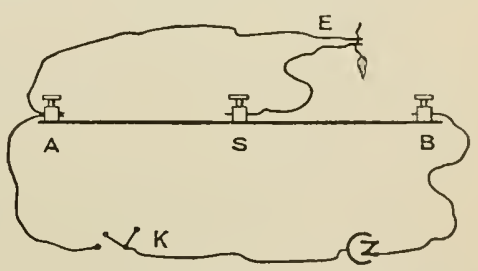

Fig. 77.-To Illustrate the PrinCIPLE OF THE MoNOCHORD. between $\mathrm{A}$ and $\mathrm{s}$. If $\mathrm{s}$ be brought nearer to $\mathrm{A}$ this potential difference is decreased in direct proportion to the distances between $A$ and $s$ in the two positions. Where, as in the case we are considering, the resistance in the nerve circuit is very high, and therefore the resistance of A s may, in comparison, be neglected, the current through the nerve is directly proportional to the potential difference only, i.e. is measured by the length $\mathrm{A}$ s.

If we wished to know exactly the value of this current, it is necessary to measure the resistances of the nerve circuit and of $\mathrm{A} B$, and from these the total amount of current ean be calculated. For most purposes, however, it is sufficient to state the current strength as measured by the lengths between $\mathrm{A}$ and $\mathrm{s}$ in different positions. 
Fig. 78 shows a monochord fitted for convenience on a small base, and consisting of a wire wound in a zigzag round pegs, its two ends being

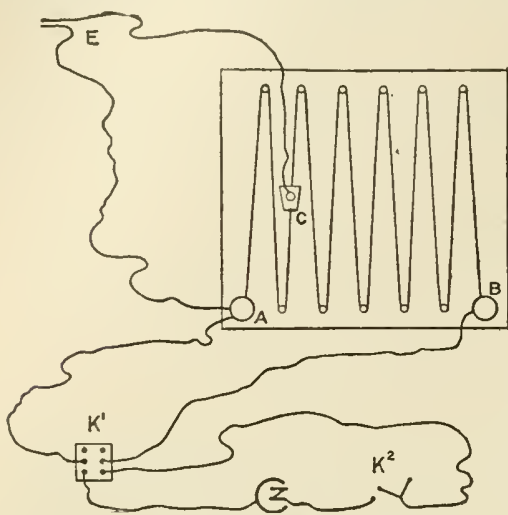

Frg. 78. -A Second Forir of Moxochord. attached to the binding screws $\mathrm{A}$ and B. The electrodes are connected to $A$ and the movable contact $C$. In the battery circuit a murcury commutator, $\mathrm{K}^{1}$, with cross wires is interposed, so that the direction of current can be readily reversed. A key, $\mathrm{K}^{2}$, is also inserted, so that the current can be closed and opened as required.

Another instrument that is frequently used for varying the strength of current is the rheochord. This consists, in its simplest form, of two wires, $\mathrm{s}^{1} \mathrm{~A}$ and $\mathrm{s}^{2} \mathrm{~B}$ (fig. 79 ), stretched parallel to one another on a wooden base. To the ends, $\mathrm{s}^{1}$ and $\mathrm{s}^{2}$, binding screws are fixed, and the wires are connected by a metal slider, c, which can be pushed along the wires. In the figure it is represented connected to send a branch current to a pair of unpolarisable electrodes across which a nerve lies. A battery current is sent through the rheochord, a commutator, $\mathrm{K}_{1}$, being interposed, and a key for making and breaking the circuit at $K_{2}$. The current on reaching $\mathrm{s}^{1}$ divides, passing throngh the rheochord to $\mathrm{s}^{2}$ or through the nerve. The amount

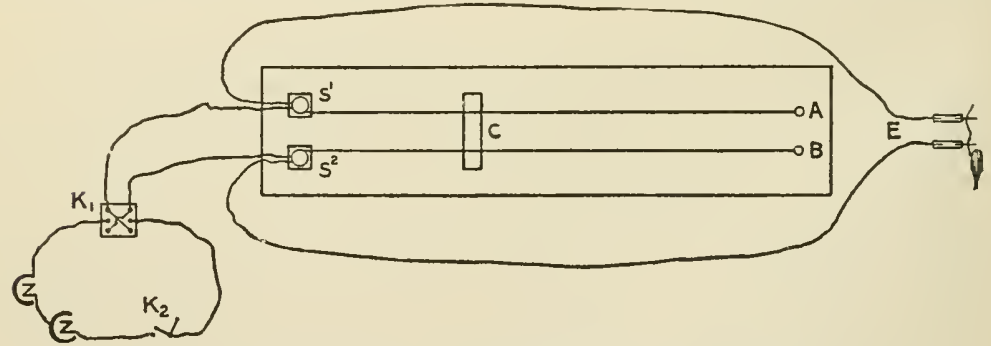

Fig. 79.-The Rheochord as Arranged for Varimg the Direction and Strexgth of a Current through a Nerve.

passing through the nerve varies directly with the resistance of its deriving circuit, the rheochord. By increasing this resistance more current is sent through the nerve, and on diminishing it less.

In more complex forms of the instrument the range of the rheochord is increased by adding other wires by means of which more resistance can be thrown into the rheochord circuit than can be reached by the single pair of wires.

Suppose, for the sake of example, that the resistance of the electrodes and nerve is $100,000 \mathrm{ohms}$, and the resistance of the rheochord 5 ohms; then $\frac{100000}{100005}$ of the total current passes through the rheochord, and $\frac{55}{10005}$ through the nerve.

Experiment 11.-Fit up the apparatus as in fig. 79. Attach the muscle to a myograph lever and arrange it to record twitches on a stationary drum. 
Start with the slider c close to the screws $\mathrm{s}_{1}, \mathrm{~s}_{2}$, in which position the current through the nerve is practically nil. Open and close the circuit by the key $\mathbf{K}_{2}$. With this very weak current no contraction occurs on make nor on break. Arrange the commutator $\mathrm{s}_{1}$ to give an ascending current, and slide $\mathrm{c}$ a little further along the wires, so as to increase the current through the nerve. At a certain position of the slider a contraction will be obtained at make but none at break. Reverse the current. The same result is obtained. A current of this length is spoken of as a weak current. It will vary in strength according to the excitability and electrical resistance of the particular nerve experimented upon. Increase the current still further, when contractions will occur on both make and break of ascending and of descending currents. A current of this strength is spoken of as a current of medimm strength. To complete the series remove the rheochord and attach the electrode wires directly to the commutator. It will usually be necessary to further increase the strength of the current to four, six, or even eight cells. Increase the current till one is found which gives a contraction on make only of a descending, and on break only of an ascending current. Snch a current is spoken of as a strong current.

The result of this experiment may be arranged in tabular form, and the statement of results is commonly termed the law of contraction. (Pfiuger's Law.)

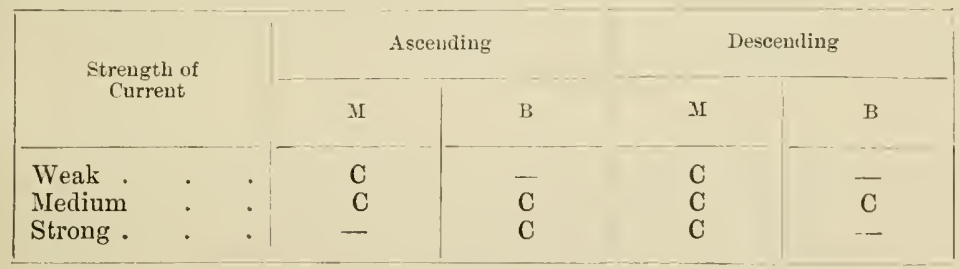

This 'law' can be explained on the basis of the facts we have already studied with regard to changes of excitability and conductivity brought about by the constant current and by our knowledge of the position at which excitation occurs. Thus, the results with a weak current mean that the production of a certain degree of katelectrotonus can stimulate, whereas the disappearance of the anelectrotonus produced by precisely the same current cannot stimulate. If the kathode be nearer the muscle (descending current) there is nothing to prevent the impulse reaching the muscle when the stimulus starts from the kathode. The muscle therefore contracts on closing the current. When the current is reversed the stimulus now has to travel through the intrapolar piece of nerve, and through that part in anelectrotonus. In both these parts the conductivity is diminished but not sufficiently to block the impulse. The muscle therefore contracts. An exactly analogous explanation holds for a descending weak current.

With currents of medium strength the disappearance of anelectrotonus can stimulate. At make of a descending current the stimulus 
starts from the electrode nearest the muscle, and there is nothing tending to block its transmission. On break the stimulus starts from the upper electrode. There is nothing which prevents it reaching the muscle, for the diminished conductivity round the kathode disappears as soon as the current is broken. With an ascending current on make the stimulus starts from the upper electrode, and as with this strength of current the block at the anode is only slight, it reaches the muscle which contracts. On break the stimulus starts from the lower electrode, and there is nothing to prevent it reaching the muscle.

With a strong descending current on make the stimulus starts from the lower electrode, and therefore leads to a contraction. On break the stimulus starts from the upper electrode, and has to travel through a piece of nerve whose conductivity is strongly depressed, sufficiently so to block the impulse, and no contraction results. With an ascending current the impulse at make starts from the upper electrode, but is blocked by the strong depression of conductivity around the anode, and therefore does not reach the muscle. On break the stimulus starts from the lower electrode, and therefore causes a contraction, as nothing prevents it travelling down to the muscle.

\section{THE VELOCITY OF A NERVOUS IMPULSE}

Just as we found that if a muscle be excited at any point the contraction begins there, and then spreads in a wave-like manner over the remainder of the muscle, so too it is found for nerve that an impulse started at any point travels along the nerve as a wave of excitation. This is brought out most clearly by a study of a motor nerve, in which we determine the latent periods of two twitches in one of which we stimulate the nerve close to the muscle, in the other at some distance from the muscle. Any difference of time in these two measurements must be due to the time occupied by the impulse in travelling from the one point to the other. The method of carrying out the experiment is as follows:-

Experiment 12.--Arrange the apparatus for recording a simple twitch, but with the drum arranged to rotate at as fast a rate as possible. Dissect out the gastrocnemius and the whole of the sciatic nerve up to the vertebrie. Place two pairs of electrodes under the nerve - one pair near to its entrance into the muscle, the other at its further extremity. Connect these electrodes $\mathrm{E}^{1}$ and $\mathrm{E}^{2}$ to the terminals of a Pohl's commutator without cross wires, as in fig. 80. Interpose a Du Bois key between the secondary coil and the remaining terminals of the commutator. The secondary circuit can in this way be connected $u p$ to either pair of electrodes, $\mathrm{E}^{1}$ or $\mathrm{E}^{2}$, by 
turning the rocker into the proper position. Take two simple twitches, recording them one over the other, stimulating the nerve $(a)$ at $\mathbf{E}^{1}$ and $(b)$ at $\mathbf{E}^{2}$. 'Take a time tracing under the record.

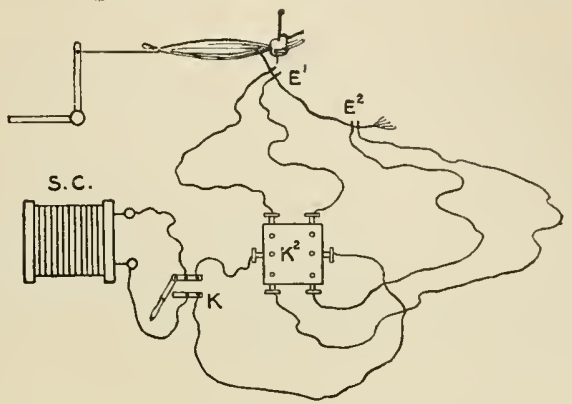

Fig. 80.-Arraygement of Apparatus for Studying the Velocity of A Neryous Impulse.

A tracing obtained in this manner is shown in fig. 81. It is seen that the latent periods differ in the two cases, the second one being longer than the first. This difference in time can only be accounted for by supposing that the molecular changes started in the nerve by the stimulation require time for their transmission along it. In the figure $a$ is the point of stimulation, $b$ is the instant at which the muscle began to contract with the electrodes in the first position, and $c$ when they were in the second position. The time tracing is at the rate of 100 vibrations per second, and on measuring it is found that the length between $b$ and $c$ corresponds to a time of 0.00369 sec. The length of nerve between the two positions of the electrodes was $74 \mathrm{~mm}$. Hence the impulse would in one second travel $\frac{74}{0.00369}=20054$

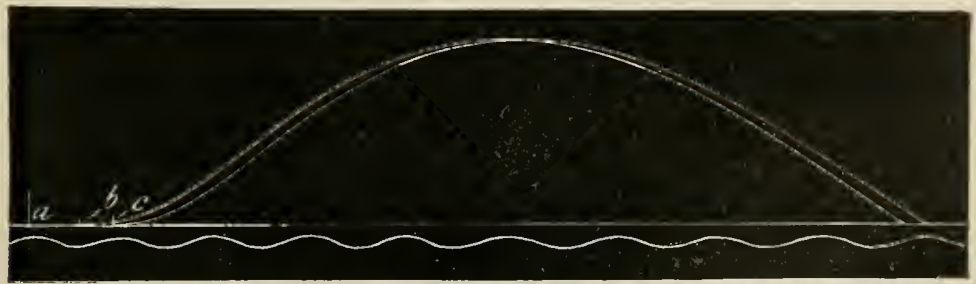

Fig. 81.-Two Twitches of a Gastrocnemius when the Sciatic was Stimelated (a) near the Muscle, (b) near the Vertebre. Thue Tracing, 100 per sec.

$\mathrm{mm}$. per sec. In this experiment, then, the velocity of the nervous impulse was 20.05 metres per second. In an experiment of this kind it is very important that the recording surface should travel at exactly the same rate when the two twitches are taken. Hence it is better. to take the record upon a pendulum or spring myograph, or two quite 
separate twitches, each with its own time tracing, may be recorded, and the difference between the two latent periods can then be calculated.

The velocity of a nervous impulse varies considerably in different animals and under different conditions. For the sciatic of the frog it is found to vary from 2 to $30 \mathrm{~m}$. per second, the chief variation being caused by differences in temperature, the rate being considerably diminished by cooling the nerve. When a very rapidly moving recording surface is not available for the preceding experiment, we may make use of this last factor to delay the rapidity of the nervous impulse, and so obtain curves which have a sufficient difference between their latent periods to make the time measure well marked. 


\section{CHAPTER II}

EXAMINATION OF THE FROG'S HEART. THE FIRST STANNIUS

LIGATURE

To examine the main features of the beat of a frog's heart proceed in the following way:-

1. Expose the heart. Pith the brain, leaving the spinal cord intact, and lay the frog on its back. Pick up the skin over the sternum and slit it up in the mid line, make transverse incisions on either side and reflect the four flaps of skin thus formed. The sternum should now be completely exposed. Make a transverse cut through the lower cartilaginous piece of the sternum (xiphi sternum), taking care not to wound the anterior abdominal vein, and cut through the sternum by longitudinal incisions a little on either side of the mid line, and thus remove the central piece. Care is to be taken not to injure the pericardium which lies beneath. The lateral pieces may now be pulled apart. This exposes the heart still lying within the pericardium. Lift up the pericardium with fine forceps and snip it through from the apex of the heart to the base.

2. Examine the different parts of the heart. Above lie the two thin-walled auricles, fig. 82 , their line of division not being clearly seen, as it is mainly hidden by the bulbus arteriosus, B A, which crosses the auricles from below upwards and from right to left. Below is the single ven tricle, bluntly pointed, and with a well-marked groove, the auriculo-ven tricular groove, separating it from the two auricles.

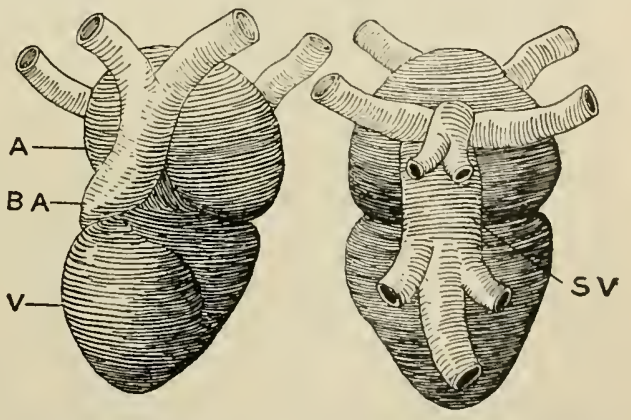

Fig. 82.-Axterior and Posterior Surfaces of the Frog's Heart. sv, Sinus Vexosus. a, Auricles. V. Vextricles. ba, Bulbus Arteriosts. If the heart be now lifted up by the apex, the lower half of the sinus 
venosus, sv, is brought into view lying in the posterior pericardial wall. At its junction with the auricle a transverse crescentic line, white in colour, is to be made out, marking the limits of the two cavities. Running from a point in the mid line of the posterior ventricular wall, and about $2 \mathrm{~mm}$. from the auricular ventricular groove, is a thin cord of fibrous tissue which is attached to the posterior pericardial wall. This is the frenum, and contains a small blood vessel. Entering the sinus from below is the single large inferior vena cava, whose outline can be made out below the pericardium. Replace the ventricle and gently displace the two auricles by means of a seeker. In this way the upper end of the sinus can be exposed with the two smaller superior venæ cavæ rumning into them.

3. Note that at each contraction the ventricle becomes pale in colour, and with each distension, which occurs very suddenly, the ventricle becomes deep red. This change is dependent on the structure of the heart wall, which has the form of a sponge-work of muscle bundles between which the blood passes during dilatation and causes the dark colour. With each contraction the blood is driven out of the spongework, leaving the muscular tissue only, which has a pale colour.

4. Determine the rate at which the heart is beating by timing it with a watch. Make a note of the number of beats per minute. It will be found to vary very greatly in different frogs-from $20 \mathrm{up}$ to 100 per minute, and in some cases even passing these limits in either direction.

5. Make out the course of the contraction as it travels over the heart. This is of some little difficulty even when the heart is beating slowly. The contraction starts at the sinus, thence passes to the auricle, then to the ventricle, and finally to the bulbus arteriosus.

6. RECORD THE BEAT OF THE HEART. One of the most convenient ways is that known as the SUSPENSION METHOD. Pre. pare a lever in the following way (fig. 83). A light straw, s, is selected, and near to one end an S-shaped hook is attached by means of a little cement. The hook may be conveniently made from a fine entomological pin. $3 \mathrm{~cm}$. from the hook the straw is transfixed by a fine needle, $\mathrm{N}$, and is then cut of such a length that when the writing point is fixed its length from needle to writing point is about $15 \mathrm{~cm}$. The magnification will then be fivefold.

The last $12 \mathrm{~cm}$. of the straw may with great advantage be replaced by a glass writing point. This is made of a piece of glass tubing drawn out to a fine capillary, which is then bent into the form of an elongated triangle, G L, by heating it in the flame of a match. The two ends at the acute angle are then fused and the knob of fused glass drawn out by another piece of glass tubing into a fine rounded point projecting at right angles to the plane of the 
triangle. It is then fixed to the end of the straw by a little cement. Such a writing point possesses the great advantage of a considerable degree of rigidity

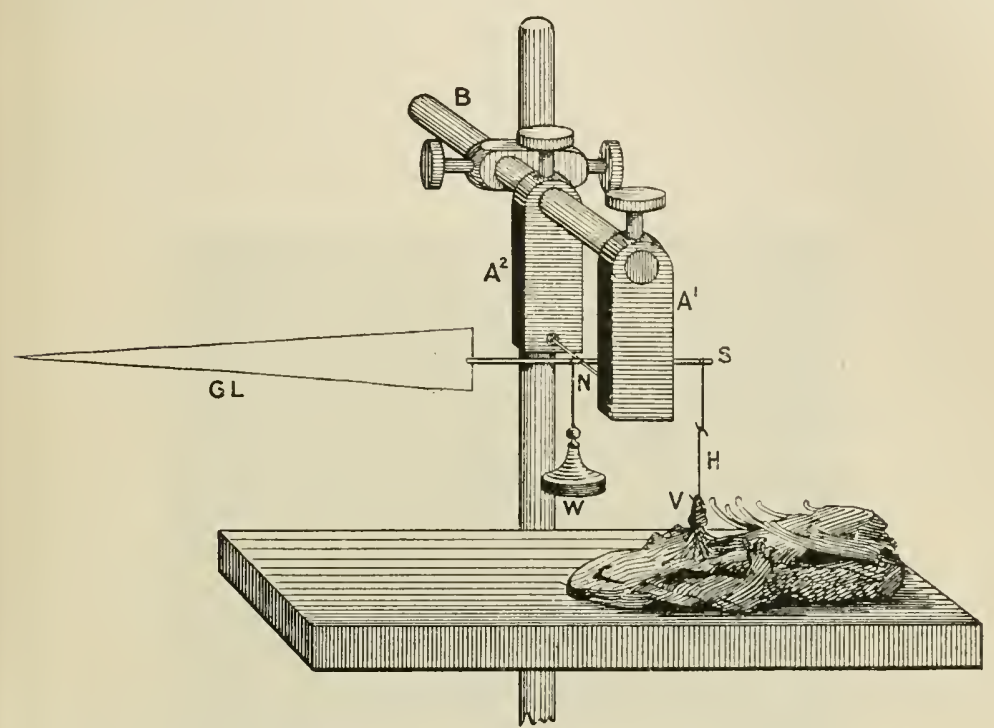

Fig. 83.-Apparatus for Recording the Heart-Beat by the Suspeision Method.

in the vertical direction with free flexibility in the horizontal direction. It is of much less weight than the straw, and owing to its lightness it is not readily broken. They are also very easily and quickly made.

The needle is now fitted into two flat brass pieces, $A^{1}, A^{2}$, which slide on a brass rod, B, and have two conical cavities at their lower ends to receive the points of the needle. The needle then acts as the axis of rotation of the lever, and the hook at the end $s$ hangs downwards.

Lift up the ventricle, $v$, of the heart which has already been prepared and transfix its apex by a sharp-pointed hook, H. Place the frog on a cork myograph plate and fix both lever-holder and cork plate on a stand, and hook the end of the bent pin fixed to the heart into the lower bend of the hook on the straw, s. With the light glass lever it will be found necessary to weight the longer arm in order to enable it to lift the ventricle up, and so exert a sufficient tension on the auricle. The weight $w$ is to be attached by hooking it on to the straw at a point about 1 to $2 \mathrm{~mm}$. from the axis.

Record the movements of the writing point upon a drum which should revolve at the rate of $1 \mathrm{~cm}$. per second, and at the same time take a 
chronograph tracing of a spring vibrating two, four, or eight times per second (fig. 29). Having recorded several beats, change the rate of the drum to $\frac{1}{2} \mathrm{~cm}$. per second, and record again. The rate may still further be reduced and so a series of tracings produced.

Fig. 84 shows some typical tracings taken by this method, but from different hearts. In I great care had been taken to avoid any loss of blood during the preparation of the heart. We see that there

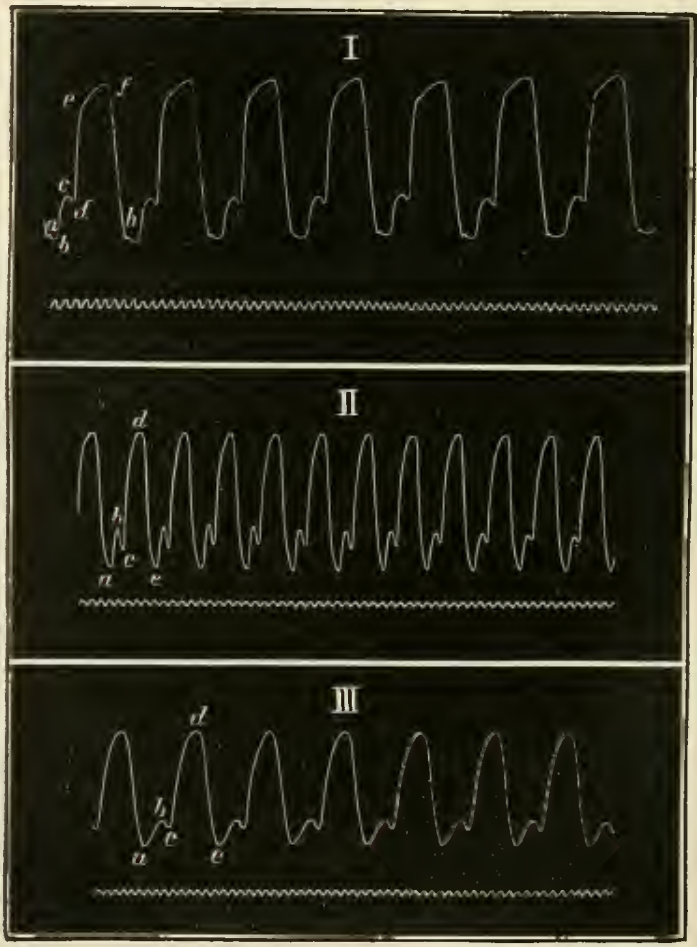

Fig. 84-Record of the Movenents of the Frog's Heart bi the Suspension Method. Tinie Tracisg, 4 per sec.

are four ascents on the curve, viz. (i.) at $a$; (ii.) from $b$ to $c$; (iii.) from $d$ to $e$; and (iv.) from $e$ to $f$. The little notch at $a$ is due to the sinus contraction. It is never very marked, and commonly is absent altogether. This is due to the position of the sinus, which does not allow it to produce much effect upon the recording lever; and secondly, because it usually occurs either during the relaxation, or even at the end of the ventricular contraction, and is therefore masked by the greater movement due to them. Even in this instance we see that 
the downward stroke due to its relaxation is more marked than the upward stroke of the contraction, which was partly masked by the ventricular relaxation. The rise from $b$ to $c$ is due to the contraction of the auricles. It is at first rapid and then gradually slows. The descent from $c$ to $d$ is due to relaxation of the auricles, and from $d$ to $e$ is the first part of the ventricular contraction. The processes occurring just before and at $d$ are in two directions. First there is the relaxation of the auricles which would allow the lever to descend, and secondly there is the ventricular contraction which causes the lever to ascend. The actual movement of the lever is the algebraic sum of these two movements, and the upward movement of the ventricle soon exceeds in amount the fall due to the auricular relaxation. The part of the curve from $e$ to $f$ is especially interesting. It also is due to the contraction of the ventricle, so that this contraction occurs in two stages. The meaning becomes clear if we cut the two aortæ when we find that the second stage, $e$ to $f$, immediately disappears. It is also only present in tracings produced from hearts which receive a full blood supply. It proves that the ventricle at first is able to empty itself comparatively easily, and therefore shortens rapidly. At the point $e$, however, the pressure inside is suddenly raised, and the contraction becomes slower. The last part of the blood is ejected with greater difficulty than the first. This receives a further support from a study of the arrangement of the blood vessels springing from the aortæ. The first and main part of the blood is sent into the pulmonary and a ortic arches, which offer a relatively low resistance. The pressure here is thus rapidly raised until it reaches such a height that it is able to overcome the resistance of the third arches, and blood then passes into the carotids.

In II and III the characters of the beat are somewhat different. In III the heart was beating slower than in II, and it is seen that the main increase in rapidity occurs during the systole. The part from $a$ to $b$ is the auricular systole; from $c$ to $d$ the ventricular. The second stage of the ventricular systole is only faintly indicated in a few beats, and in most it is quite absent. In a quite bloodless heart this second stage is entirely absent (see, for instance, fig. 100, p. 123).

7. EXCISE THE HEART. Remove the frog from the myo. graph plate and take away the hook from the ventricle. Count the number of beats in a minute. Cut through the two aortæ close to the bulbus if they have not already been severed. Lift up the ventricle by the apex and cut through the pericardium, so as to expose the sinus venosus; cut through the inferior cava and the two superior cavæ as they come into view, and thus remove the whole heart as in fig. 82. 
Place it upon a clean glass plate. The process of cutting will be found to exert an influence upon the rhythm. Usually it is inhibited for the space of a few beats and then recovers and beats very rapidly for a short time, but quickly settles down to a regular rate, which, as a rule, is slower than that in situ, though the reverse may at times occur. When it has settled down count and make a note of this rate per minute. Cut away the sinus at its junction with the auricles. The effect upon the auricles and ventricle is that they give a series of rapid beats and then come to a standstill completely relaxed. The effect upon the sinus is usually found to be inhibitory. It stops beating at once, though in a few cases, if care be taken to injure it as little as possible, it may continue its rhythm unaltered. After a short period both parts recommence to beat, but with quite a different rhythm. Count the rate per minute. The auricles and ventricle beat at a rather less rapid rate than the whole heart. The sinus being more injured takes longer to recover and beats at a slower rate. If the course of the experiment be modified, in that instead of removing the sinus with the rest of the heart the incision be made between sinus and auricles, so as to leave the sinus in situ, it is usually found that the sinus beat is from the first but little affected, and frequently quite unaltered. Next remove the auricles from the ventricle, cutting on the auricular side of the auriculo-ventricular groove so that a small edge of auricle is still attached to the ventricle. The process of cutting again inhibits both auricle and ventricle. After a time both recommence to beat the auricle earlier than the ventricle. If the amount of auricle still attached to the ventricle be very small the ventricle may not of itself recommence beating. If it do not, it may be taught to beat by stimulating it rhythmically with mechanical (e.g. a prick of a needle), or with electrical stimuli repeated at regular intervals. In many cases it will then be found that the ventricle starts off beating quickly and gradually slows down until it ceases altogether. The rhythm may then be again started by rhythmic stimuli. If the ventricle recover its rhythm cut through it just below the auriculo-ventricular groove; the ventricle is brought to a standstill and does not regain an automatic beat if left to itself. With each mechanical stimulus a contraction follows, and if the stimuli be repeated rhythmically for a time, say one per second, the ventricle may regain an automatic rhythm, though this does not often occur with a frog's heart. If it should, count the rate.

The results of this experiment should be collected together in a tabular form somewhat after the plan adopted in the following experiment, which is slightly modified from the preceding instructions:- 


\begin{tabular}{|c|c|c|}
\hline Time & Observations & Rhythm \\
\hline 11.39 & Heart exposed. Pericardium still unopened & $\begin{array}{c}\text { V. A. s. } \\
62\end{array}$ \\
\hline 11.44 & Immediately after opening of pericardium . & 51 \\
\hline 11.45 & $\begin{array}{l}\text { Auricles }(\mathrm{A}) \text { and ventricle }(\mathrm{v}) \text { excised, leaving sinus }(\mathrm{s}) \text { in situ. } \\
\mathrm{s} \text { slowed for a few beats, } \mathrm{A} \text { and } \mathrm{V} \text { also inlibited for two to } \\
\text { three beats }\end{array}$ & \\
\hline 11.46 & $\begin{array}{c}\text { three beats } \\
\text { s beat counted }\end{array}$ & 53 \\
\hline 11.47 & $\mathrm{~A}$ and $\mathrm{v}$ beat counted & 35 \\
\hline 11.50 & s beat counted. & 54 \\
\hline 11.51 & $\mathrm{~A}$ and $\mathrm{v}$ beat counted & 38 \\
\hline 11.52 & s excised. Very much slowed but not stopped. & \\
\hline 11.54 & $\begin{array}{l}\text { A cut ofi, leaving a rim attached to the ventricle. v does } \\
\text { not stop, but the rhythm becomes irregular, and beats occur }\end{array}$ & \\
\hline & in groups of three. A stops. $\mathrm{r}$. & 36 \\
\hline 11.55 & A has recommenced. & 42 \\
\hline 11.56 & $\begin{array}{l}\text { s beat counted. } \\
\text { The ventricular beat obviously follows the beat of the piece } \\
\text { of A still attached to it }\end{array}$ & 54 \\
\hline 11.58 & $\begin{array}{l}\text { v apex cut off. Auricular piece still goes on beating. v } \\
\text { apex responds to each prick of a blunt needle, but cannot } \\
\text { be taught to beat rhythmically. }\end{array}$ & \\
\hline
\end{tabular}

There are several conclusions to be inferred from such experiments.

A. The excised heart beats rhythmically. Therefore the rhythm is not dependent upon external rhythmic stimuli reaching it via its nerves, but is due to some intrinsic source possessing a rhythmic activity. There are two possibilities. (a) The rhythmic activity may be an inherent property of the muscle fibres. (b) The heart contains in itself nerve cells and nerve fibres; and the rhythm may be a characteristic of some of these cells. By this experiment taken alone we are not able to prove which of these two possibilities is the correct one. The last part of the experiment, if it succeed, is the most helpful in deciding, but the frog's heart is not a very suitable subject to employ for this particular purpose. On a tortoise heart Gaskell has shown that the apex, which examination proves to be free from nerve cells, can be taught to beat rhythmically. Further facts tending to prove that the rhythm is inherent in the muscle cells are: (i.) the heart beats rhythmically in embryonic life, while it is still of tubular form and before it has gained any nervous connection with the central nervous system; (ii.) nicotine has been proved to paralyse nerve cells, but a heart poisoned with nicotine continues to beat rhythmically; (iii.) there is no difficulty in ascribing the rhythm to the heart muscle, for cross-striated muscle may be caused to contract rhythmically by immersing it, under proper tension, in Biedermann's fluid, and the bulbus arteriosus, which contains no ganglion cells, beats

' Biedermann's fluid is made by dissolving 5.g. $\mathrm{NaCl}, 2 . \mathrm{g} . \mathrm{Na}_{2} \mathrm{PO}_{4}$ and 0.59 .g. $\mathrm{Na}_{2} \mathrm{CO}_{3}$ in 1 litre of water. 
rhythmically when completely isolated from all other parts of the heart; (iv.) an isolated heart apex can more readily be made to beat rhythmically if it be subjected to an internal pressure, as by tying it on to a perfusion cannula through which fluid is circulated at sufficient pressure.

B. Taking it as proven that the rhythm is a property of the muscle cells, it follows that the cells in different parts do not possess the rhythmic capacity to an equal degree. The sinus, if left intact in the body, beats with the same rhythm as the intact heart, and this, taken in conjunction with the fact that the contraction wave starts normally from the sinus, shows that in the sinus rhythmic activity is especially developed. That the auricles and ventricle, when severed from the sinus, beat at a slower rate is generally taken as indicating that the auricles, the part from which the contraction wave starts when the auricles and ventricle are removed, possess rhythmic characters to a lesser degree than the sinus. Finally in the ventricle rhythmic activity is still less developed, and with the ventricle apex can only be demon. strated under especial conditions.

Experiment 2.-The first Stannius ligature. Pith a frog and expose its heart, cutting away the pericardium, so as to thoroughly expose the auricles and two aortæ. With an aneurism needle pass a thread under the bulbus arteriosus and above the two superior cava. Lift up the apex of the heart, cut through the frenum, and bring the ends of the thread round the heart, so that it lies under the auricles. Tie a loop in the thread and tighten it, so that it lies over the crescentic junction of simus and auricles after the ligature is tightened. The auricles and ventricle usually give a few beats and then come to rest in a fully relaxed condition, whilst the sinus continues to beat at the same rate as before. At times the auricles and ventricle still continue beating, though at a slower rate. This is usually due to the ligature being wrongly applied. It should be relaxed and tied a second time a little nearer to the auricle, when the required standstill will usually occur.

In fig. 85 the effect of the application of the Stannius ligature is

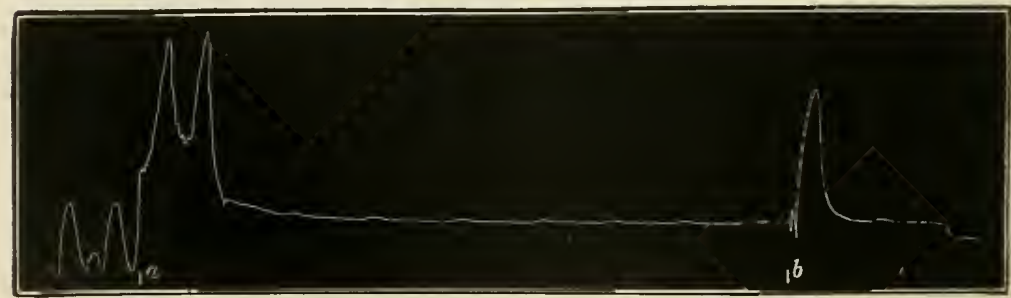

Fig. 85.-Application of the First Stannius Ligatore to the Frog's Heart at the Point $a$. At $b$ a Beat due to a Mechanical Stimulus.

recorded graphically. At the point $a$ the ligature previously placed in position was suddenly tightened. The two peaks immediately 
recorded are mechanical and due to the process of tying. The auricle and ventricle beats stopped at once, and the recorded line only shows slight undulations, which were caused by the contraction of the sinus. The rhythm of the sinus is seen to be at first slowed. At the point $b$ the ventricle was stimulated by a prick of a needle. It gave a single contraction.

Prick the auricle. With each stimulus the quiescent part gives a single beat. Repeat on the ventricle. The same result is obtained. Next stimulate with an induced shock. A single contraction is also obtained in this way.

The Stannius ligature is of great importance because it enables us to study a single contraction after the same method adopted in studying a single twitch of a muscle, whereas while the heart is still beating rhythmically this is impossible.

Experiment 3.-Test the response of the heart to electrical stimuli of different strengths. Attach the apex of a heart brought to a standstill by the Stannius ligature to the recording lever, as in fig. 83. Remove the secondary coil to such a distance from the primary that neither make nor break shocks excite a contraction. Bring the writing point to the surface of a drum at rest, so as to record heights of twitch only. Move the secondary coil gradually up to the primary, stimulating the heart with each new position until one is found at which a contraction occurs at break. Move the drum 1 or $2 \mathrm{~mm}$. by hand. Bring the secondary $1 \mathrm{~cm}$. nearer the primary, and, allowing fully 30 seconds to elapse, stimulate once more. The height of the contraction is the same as before. Further increase the strength of the stimulus and record the contractions. There is no increase in the beight.

Therefore a minimal stimulus causes a maximal contraction. If a heart contracts at all it contracts to its full power.

The reason for allowing an interval of from 30 to 60 seconds to elapse in the preceding experiment before applying a second stimulus is because a second stimulus of even the same strength is found to produce a greater effect than the first if it follow the first within a second or so. This can be shown in the following way :-

Experiment 4.-Arrange the drum to rotate at a very slow rate, about $2 \mathrm{~cm}$. per minute. Apply electrical stimuli every five seconds, recording the contractions on the moving surface. Record some twenty contractions in this way.

Fig. 86 shows the result obtained. The second contraction is seen to be higher than the first, the third than the second, and so on up to the fifth, from which time they all reached practically the same height. The increment of height of the second over the first is greater than the increment of the third over the second, and this increment gradually decreases until it vanishes. This increase of effect on repeating the stimulation, keeping to the same strength, is spoken of as the staircase 
effect. We have already found that skeletal muscle gives a similar result (pp. 63 and 69).

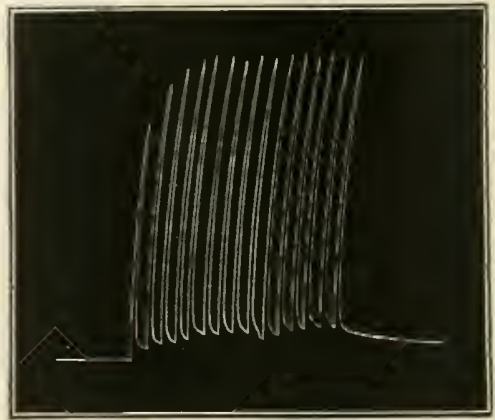

Fig. 86,-Electrical Stinulatiox of the Ventricle of the Frog's Heart in Standstml by the Stankius Ligature to Show the 'Stair zase' Effect. INTERyal betweex the Stnudi 5 secs.

Experiment 5.-Record a single beat of the heart. Arrange the drum to rotate at the rate of $2 \mathrm{~cm}$. per second. Fit up the apparatus as for recording a single muscle twitch (fig. 37). For electrodes in the secondary circuit make a pair with fine silk-covered wire as in fig 87. Cement the two wires together by a touch of sealing-wax or marine glue at a point, $\mathrm{c}$, near to their

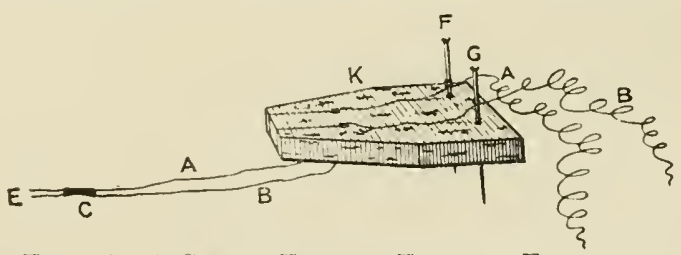

Fig. 87.-A Sinple Form of Flexible Electrodes.

free ends. Scrape off the insulation from their projecting ends, E, which should be cut of equal length. Take a piece of flat cork of the shape of $\mathrm{K}$ and pass two pins, $F$ and $G$, through it. Slit up the cork in two places at its apex and pass the wires throngh these slits by which they are held firmly; then wind each wire round one of the pins and attach the two ends to the Dn Bois key in the secondary circuit. Apply a Stannius ligature to a heart and, passing a bent pin through its apex, attach it to the recording lever, as in fig. 83 . Twist the electrodes, $\mathrm{E}$, until the wires lie above one another and pin down the electrodes to the cork base of the myograph, so that the wires tonch the base of the ventricle. Arrange a chronograph vibrating thirty times per second to record directly under the heart lever. Bring the writing point to the surface and record a contraction in the same way as if recording a simple muscle twitch. Mark the point of stimulation and draw vertical lines with the recording lever to mark on the time tracing the point of stimulation and the points (1) when contraction begins, (2) when it reaches its maximum, and (3) when relaxation ends. Draw a zero abscissa line. Repeat the experiment, varying the rate of the recording surface.

The tracing obtained is of the form shown in fig. 88 , and presents 
features closely analogous to those of a simple muscle twitch. The great difference lies in the time occupied. The time measurements are seen to be-

(a) The latent period, 0.117 sec.

(b) The period of contraction, 0.767 sec.

(c) The period of relaxation, 1.067 sec.

In examining the general form of the curve it is seen that the top tends to be flattened; a condition which is often more prominent than in this particular curve (see curves of fig. 91). In different hearts the form of the curve varies considerably, differences which are largely due to the different temperatures at which they are examined, and secondly to the time of the year. The flattening of the top of the curve tends to show that the contraction wave possesses so slow a rate that the first fibres affected return to rest before the wave has extended over all the heart fibres. This follows from the same considerations which we have already gone into when discussing the features of the contraction wave in muscle (p. 73).

In figs. 89 and 90 we have two further tracings taken from different hearts, and with the recording surface moving at different rates. In these figures the relaxation of the heart is seen to be more rapid than the contraction; a condition which is commoner than the reverse effect recorded in fig. 88. In both these figures the first and major part of the relaxation is most rapid; it then becomes much slower and gradually ceases. Note

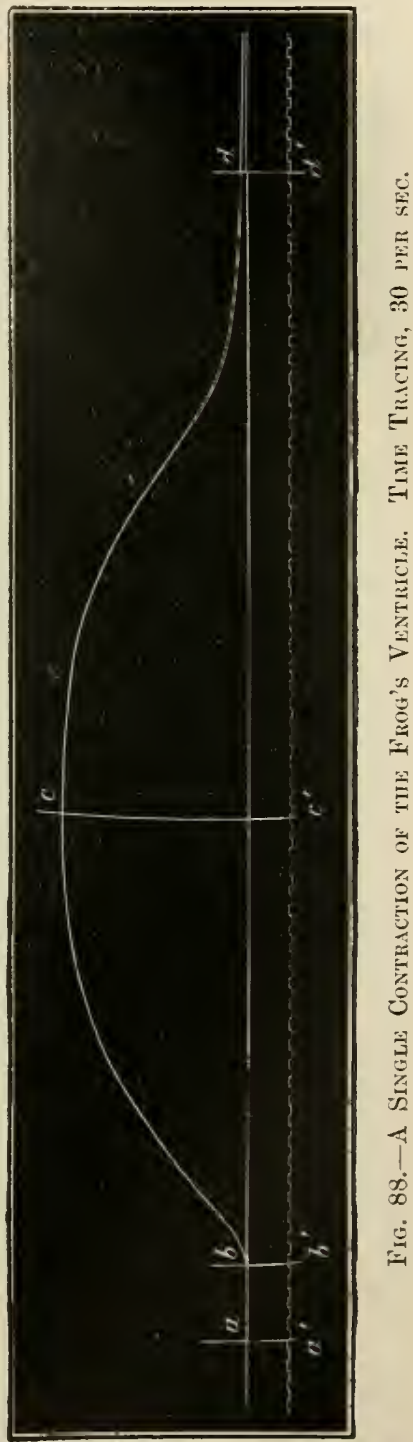
that the apex of each curve is very blunt, even flattened, indicating that the contraction is sustained for some time. 
Upon a heart brought to a standstill by the Stannius ligature we are further able to study the effect of two successive stimuli, which enables us to demonstrate one of the great physiological characteristics of heart muscle, namely, its long refractory period.

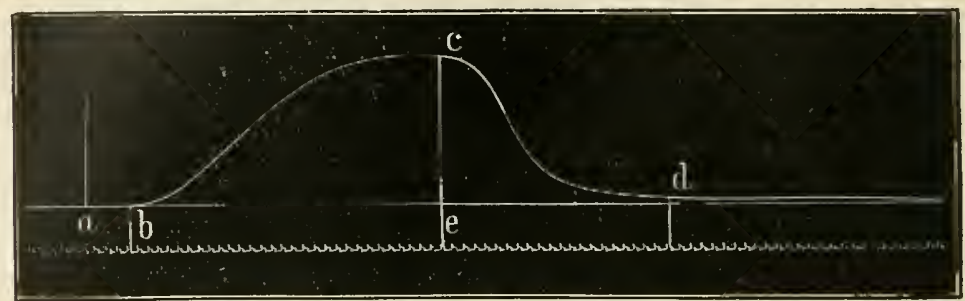

Fig. 89.-A Single Contraction of the Frog's Ventricle. Tiae, 30 per sec.

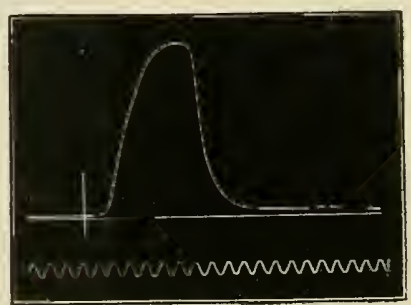

Fig. 90.-A Piepetition of the Tracing of Fig. S9 with a Slower

Movenent of the Recording Surface. Tine, Tracing, 8 per sec.

Experiment 6.-Arrange the drum as for recording the effect of two successive stimuli on muscle (fig. 58). The drum should be so geared that it rotates at the rate of 2 to $3 \mathrm{~cm}$. per second. Pith a frog, expose its heart, and apply the Stannius ligature. Pass a bent hook through the apex of the ventricle and attach to the recording lever, fig. 83 . First remove one of the contacts and record a single beat as in the previous experiment. Mark the point of stimulation and draw an abscissa line. Now arrange the second contact at such a distance behind the first that the second point of stimulation will fall near the end of the period of relaxation and record the result of the double stimulation. Mark the points of stimulation on the curve. Move the two contacts nearer and repeat the stimulation. Carry out the process until a distance between the points of stimulation is reached, when only a single contraction results.

In this way tracings such as those reproduced in fig. 91 are obtained.

Curve I. gives a record of the single beat. The time from the point of stimulation to the highest point of the curve is $\frac{2}{3} \frac{0}{0}$ ths sec. The total duration is $\frac{4}{3} \frac{5}{0}$ ths sec.

Curve II. This is the result of two successive stimuli, the second following the first after an interval of $\frac{3}{3} \mathrm{~s}$ ths sec. It therefore fell near 
the end of the period of relaxation. We see that the heart responded by giving a second beat, but that there was no real summation of the contraction. The apex of the second contraction is at exactly the same height as that of the first.

The one difference observable is that the second contraction is carried out a little quicker than the first. There is less flattening of the apex. The measurements of the 'apex time' show this very clearly. They are $\frac{31}{30}$ ths sec. and $\frac{2}{3} \frac{7}{0}$ ths sec. respectively.

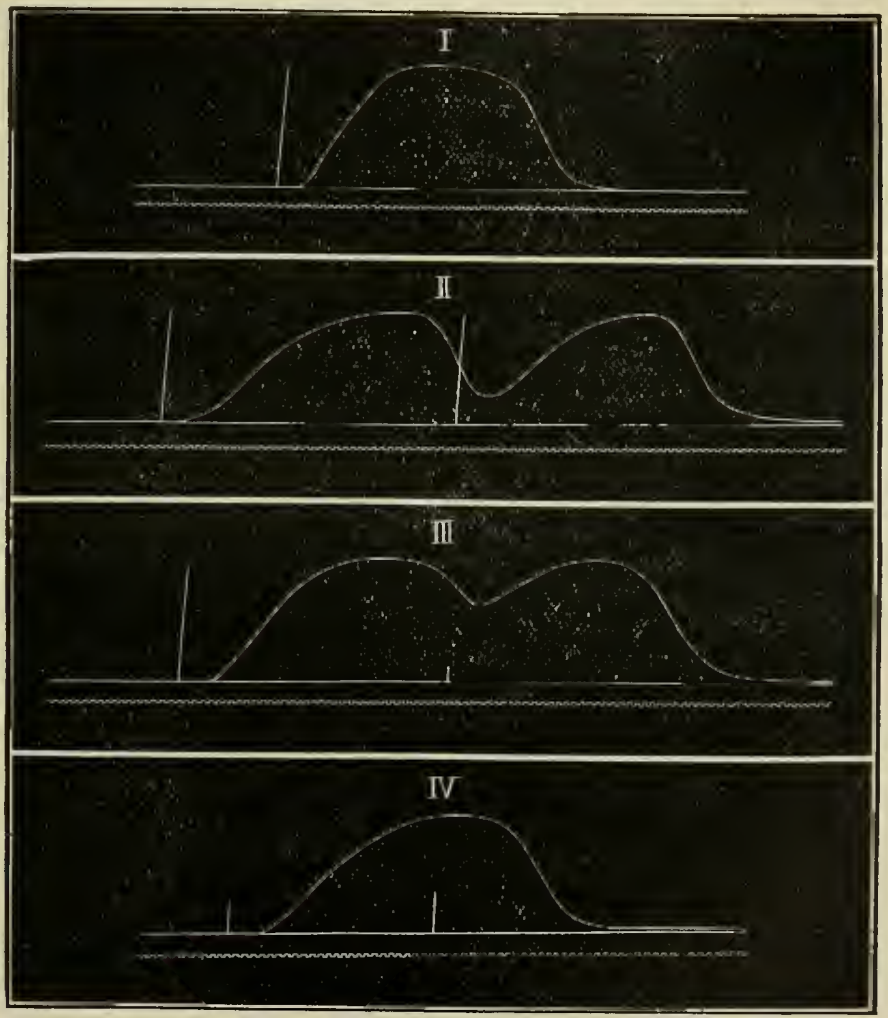

Fig. 91.-The Effect of Two Successive Stimuli upon the Ventricle of the Frog's Heart. Time Tracisg, 30 per sec.

In curve III. we have practically a repetition of curve II., but the second point of stimulation falls a little earlier, $\frac{3}{3}$ th ths sec. after the first, and therefore nearer the commencement of the period of relaxation. Again we find no true summation of height. The second contraction repeats the first, but starts with many fibres already con- 
tracted. As in the previous curve the second is rather more rapid than the first contraction. The apex times for the two are $\frac{28}{30}$ ths and $\frac{24}{30}$ ths sec.

Curve IV. The second stimulation in this instance follows the first

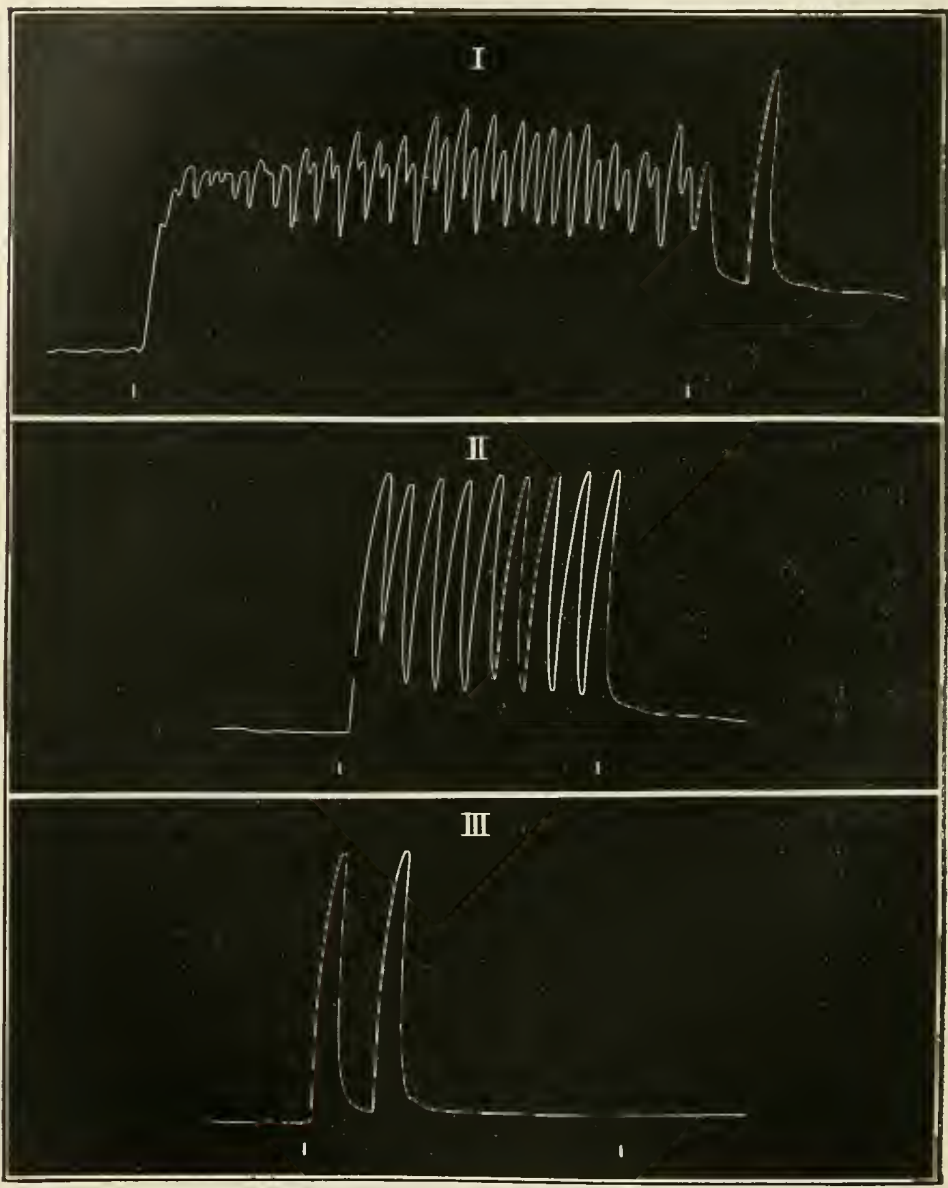

Fig. 92.-Tetanisation of a Frog's Ventricle in Standostill BT the Stannzus Ligatore. IN I the Secondary CoIL at 10 chs.; IN II at 12 ciss.; IN III at 13 chis. from the Primary. Magnification, 10. The Duration of Tetanisation is Indicated by the Short Vertical Marks under Each Tracing.

after an interval of $\frac{24}{30}$ ths sec., i.e. at the end of the period of contraction. We note the most striking result that no second contraction is produced. It has fallen on the muscle fibre during a time when it is 
unable to respond to a stimulus. That time during which a stimulus produces no response in a muscle fibre is termed its refractory period. We see, then, that for the heart the refractory period lasts through the whole of the period of contraction. In contrast with this we have previously found that the refractory period for skeletal muscle was practically identical with its latent period.

Experiment 7.-Upon the same heart study the effect of a rapidly repeated series of stimuli. Arrange for the recording surface to more very slowly. Set up the coil for giving tetanising shocks, and then stimulate the heart, varying the strength of stimulus employed.

In fig. 92 are shown three curves obtained in this way with different strengths of stimulus. In I the stimulus was strongest, and we see that during the whole period of stimulation, lasting during the interval between the two vertical marks, the ventricle never completely relaxed. The important result, horvever, is that there is no complete fusion. A heart cannot be sent into complete tetanus. At first the amount of fusion was marked, but as the stimulus proceeded fusion became gradually less, and a rhythmic contraction, which was not very regular, was produced. Putting it in another way, we may say that as tetanisation proceeded the refractory period tended to increase. Note, moreover, that a contraction occurred after stimulation had ceased, showing that the effect of the stimuli did not stop immediately stimulation ceased. Tracing II was taken from the same heart, but the secondary coil was removed $2 \mathrm{~cm}$. further from the primary. With this weaker stimulation there is no summation of effect, except in the case of the first two beats. A rhythmic beating was started which ceased on the stimulation being stopped. In the third curve (III) the stimulus was still weaker, and only two contractions were produced at the commencement and none during the rest of the time of stimulation.

A single break shock with the secondary coil at $13 \mathrm{~cm}$. produced no response, whereas at $12 \mathrm{~cm}$, the position during tracing II, a contraction was produced. 


\section{CHAPTER X}

\section{THE ACTION OF HEAT AND COLD UPON THE FROG'S HEART}

\section{THE EFFECT OF HEAT AND COLD UPON THE EXCISED BLOODLESS HEART}

Experiment 1.--Pith a frog and expose its heart. Cut through the frenum and pass a bent hook through the apex of the ventricle. Cut away the lower jaw and now remove the heart entirely with the pericardium, cutting out with it the surrounding tissues pretty freely. Pin this on to a cork base fixed to a metal bar made in the following way (fig. 93). Select a round flat cork and bore throngh it from the side, making a hole sufficiently large to fit tightly on the short arm of a brass L-piece. Fix the cork to the L-piece with a little sealing-wax. The pins should pass throngh the floor of the pericardium, so as to fix the heart firmly to the cork. The heart can

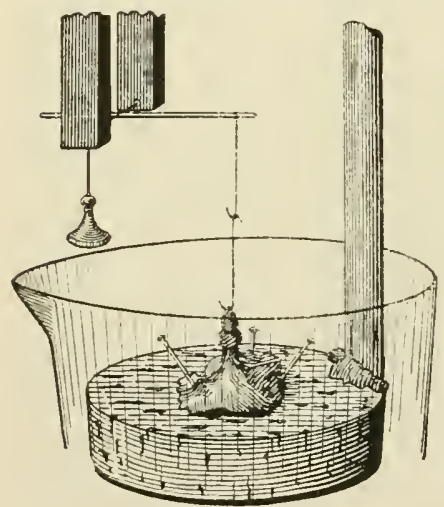

Fig. 93. be titted to the recording lever as in the previous experiments (see fig. 83). A small beaker is then filled with defibrinated ox-blood, diluted with four times its volume of normal saline, or with Ringer's solution, ${ }^{1}$ which has previously been cooled in a freezing mixture to about $0^{\circ} \mathrm{C}$. The writing point is brought to the surface and a chronograph marking seconds arranged to write immediately under it. The drum is set in motion, and after a short length of tracing has been taken the beaker of cooled fluid is raised so as to immerse the heart. The character of the tracing is at once altered, and after about half a minute, when the record no longer changes, the fluid is lowered and the gradual change in the beat, as the temperature returns to its original height, is recorded. Take several tracings in this way, raising the temperature of the fluid five degrees for each fresh tracing.

Tracings obtained in this way are reproduced in fig. 94. In the first the heart was suddenly immersed in diluted blood at $4^{\circ} \mathrm{C}$. The following beat is seen to be of less height and considerably prolonged, the second one of greater height, and then the heart settled down to a slow rhythm of considerable force in which the contraction was sustained at its height for some time, and relaxation was considerably prolonged.

1 Ringer's solution is made by saturating $0 \cdot 65$ per cent. $\mathrm{NaCl}$ solution with calcium phosphate and then adding 2 c.c. of a 1 per cent. solution of potassium chloride to each 100 c.c. 
The rate before cooling was 24 per minute; during cooling, 7 . After removing the cold blood the heart very rapidly increased in rate, and for the first few beats in force. The rate was increased until the beat became faster than the original rate. The force gradually decreased until at last both rate and force after about five minutes returned to their original state.

At a rather higher temperature $11^{\circ} \mathrm{C}$. the same effects are observed, though to a much less marked degree (fig. $94 \mathrm{~A}$ ).

At $25^{\circ} \mathrm{C}$. it is seen that the rate is greatly increased-from 51 to 93 per minute. The force of the ventricular and auricular contractions remains practically unaltered, but the time of each is markedly diminished, so that the auricular relaxation is finished before the ventricular contraction begins. On removing the warmed blood these changes gradually disappear.

If a heart be immersed in blood at about $35^{\circ} \mathrm{C}$., fig. $94 \mathrm{~B}$, a very interesting result is obtained. The ventricular contraction at once ceases, but the auricular persists-at first very weak, but gradually becoming stronger. If immersion be prolonged one beat is dropped at irregular intervals, at times two successive ones. On cooling, the heart, after some few seconds, once more begins to beat, and at first with greatly increased frequency. Sometimes, as in the tracing reproduced, the ventricular contractions after a time are once more dropped, and the auricular become much weaker. After a varying interval, rhythm for a second time returns, and the heart apparently recovers completely. During both periods, when the ventricular contractions cease, the ventricle will respond with a single beat on mechanical or electrical stimulation, though the stimulus required is greater than normal.

Another change which this tracing shows is in the amount of 'tone' of the heart. Directly after immersion the heart begins to elongate, at first fairly rapidly, and then more slowly, i.e. there is a diminution of tone. As soon, however, as the heart recommences beating this tone is recovered, and even becomes greater than before. When the ventricle once more ceases beating the tone immediately falls again.

In some hearts a different result is produced at this temperature. The heart may at first beat very rapidly before the ventricle finally stops. This is because the heart takes a little time before it reaches the temperature at which the ventricle stops, and if the fluid be only just above this temperature the time required to reach this point is much longer than if the temperature be distinctly higher.

In the last tracing the immersion fluid was at $38^{\circ} \mathrm{C}$., and it is seen that after one short contraction the ventricle ceased, and then some five or six beats later the auricle also stopped. The heart then relaxed a little, i.e. there was a diminution of tone. About 30 secs. after im- 


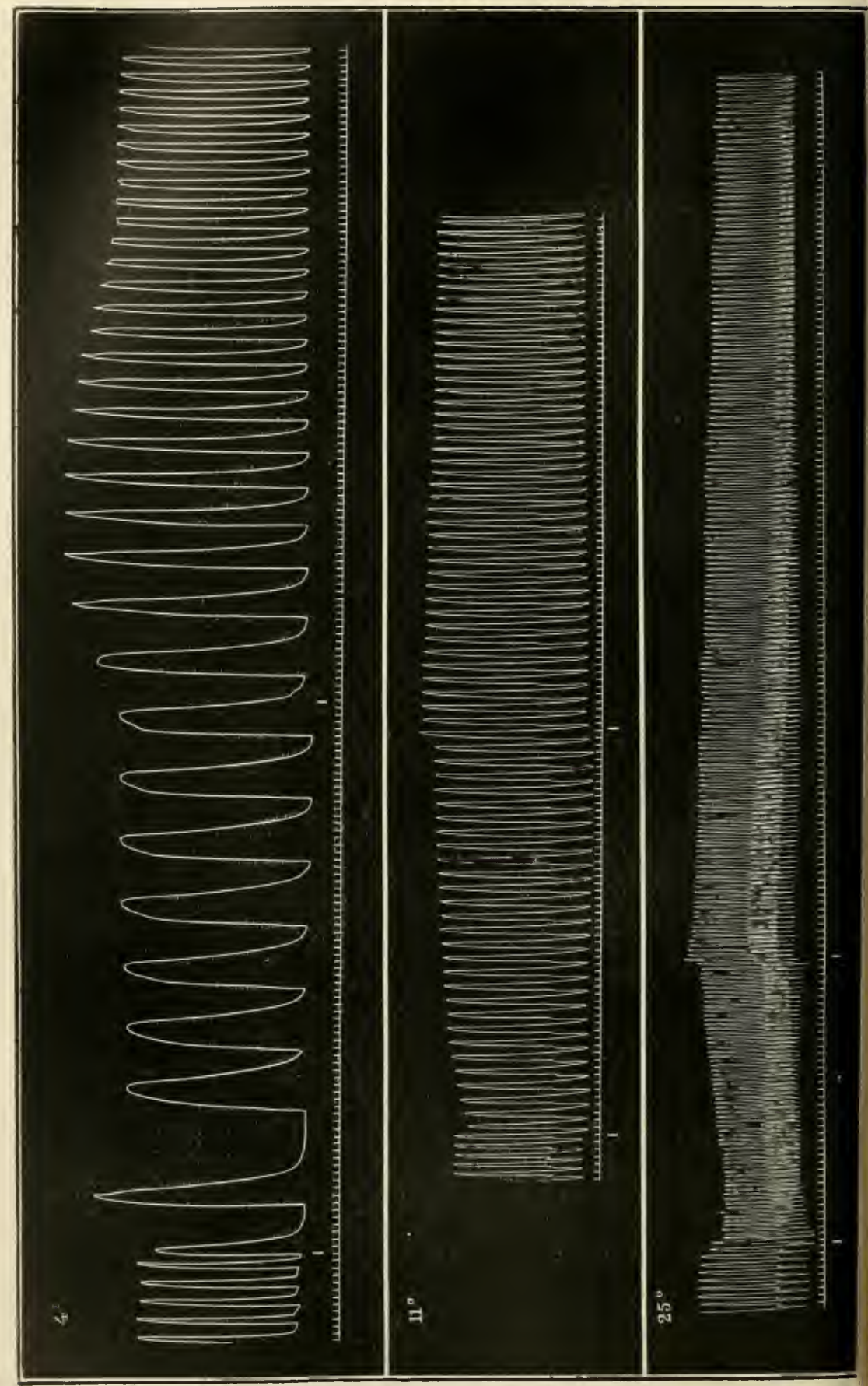


mersion a new change occurred, viz. a gradual and regular contraction. This is the production of heat rigor. The heart was kept at this tem-

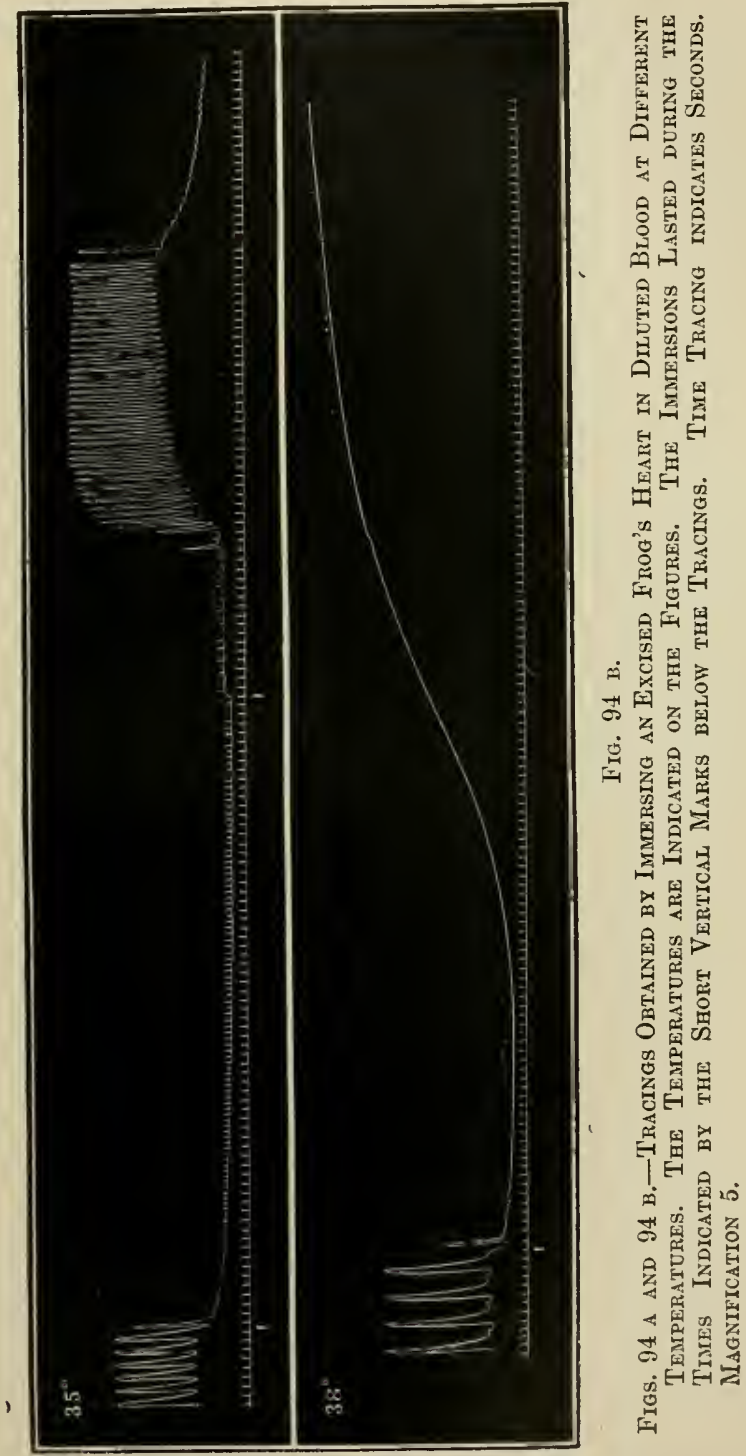

perature for four minutes and was then cooled. No relaxation occurred, though after three minutes the heart once more regained a rhythm though of much weaker force than before. 
If the heart be kept much longer at this temperature, or for a shorter time at a higher temperature, heat-contraction is more pronounced, becomes complete, and then no recovery occurs on cooling.

The effect of heat and cold upon the heart in situ.-The effect upon the beat of raising or lowering the temperature of the whole heart has been determined by several methods. We can employ the same arrangement as that of the previous experiment, but having pithed the brain it is best to curarise the frog. It is then pinned down to the cork and the heart attached to the lever. The beaker of fluid is then brought up as before, allowing the legs to remain out of the beaker. The results obtained by this method are practically the same as those already described above.

Engelmann passes a tube through the osophagus and out of an opening in the stomach wall. Water of different temperatures is then circulated through the tube.

Another simple plan is to arrange the heart for recording by the suspension method, and then while the tracing is being taken a fine stream of normal saline at the temperature required is directed on to the heart through a glass tube drawn out to a fine capillary orifice. By this method it is possible to limit the cooling or heating mainly to the atricles and sinus without affecting the ventricle.

A third plan is to record by the suspension method, having previously arranged a coil of platinum wire around the heart. The coil is then heated by an electrical current, and thus forms a small source of heat, whose action may, by altering its position, be limited chiefly to either the sinus and auricles or to the ventricle.

The results given by these methods are similar to those already given for the excised heart, but with the difference that where the alteration of temperature is limited chiefly to the sinus, the result tends towards alteration in rhythm without alteration in the character of each beat. On the other hand, where the heating or cooling effect is chiefly localised to the ventricle the main effect is an alteration in the force and character of the beat with no change in the rhythm. As in the first experiment, when the rhythm alone is altered, heat tends to accelerate; when the character is altered, heat up to about $20^{\circ}$ tends to increase the force, above that temperature to decrease the force, until at about $30^{\circ}$ the rhythm is stopped, though on again cooling the heart will recover. At about $35^{\circ} \mathrm{C}$. the heart begins to pass into the state of heat-rigor, and no complete recovery is then possible.

\section{THE EFFECT OF HEAT AND COLD UPON A SINGLE CONTRACTION OF THE VENTRICLE}

In addition to examining the result of varying the temperature upon a heart beating rhythmically the alteration in a single beat should 
also be studied a little more closely. This can readily be done by the aid of a heart brought to a standstill by the Stannius ligature.

Experiment 2.-Prepare the apparatus for recording single contractions as in experiment 5, p. 106. The drum should rotate $2 \mathrm{~cm}$. per second. Pith a frog, expose its heart, and apply the Stannius ligature. Hook a bent pin through the tip of the ventricle apex. The heart may now be excised and attached as in fig. 93. Fix the electrodes for stimulating the ventricle. Take a small beaker full of the diluted blood (or Ringer's solution), which has previously been cooled in a freezing mixture, and bring it up around the cork base, so as to immerse the heart. Take the temperature of the dilute blood. After about a minute's immersion lower the beaker and record a contraction. Raise the temperature of the dilute blood five degrees by placing the beaker for a few seconds in hot water. Again immerse the heart for one to two minutes. Then lower the beaker, see that the writing point is again at the same level, and record a second contraction over the first. In this way record a series of contractions, increasing the temperature each time until a range of from $5^{\circ} \mathrm{C}$. to $30^{\circ} \mathrm{C}$. has been reached. Mark the point of stimulation, draw an abscissa line, and take a timetracing of 30 per second beneath the zero abscissa.

Fig. 95 represents such a series of curves. Measurements of each should be taken and arranged in a tabular form, as has been done for this experiment in the table on next page.

From this figure and its accompanying table the following points are clear :

1. As the temperature rises the latent period becomes shorter.

2. As the temperature rises the period of contraction becomes shorter, at first very rapidly and then more slowly.

3. The period of relaxation shows a sudden shortening from 7 to $10^{\circ} \mathrm{C}$. It

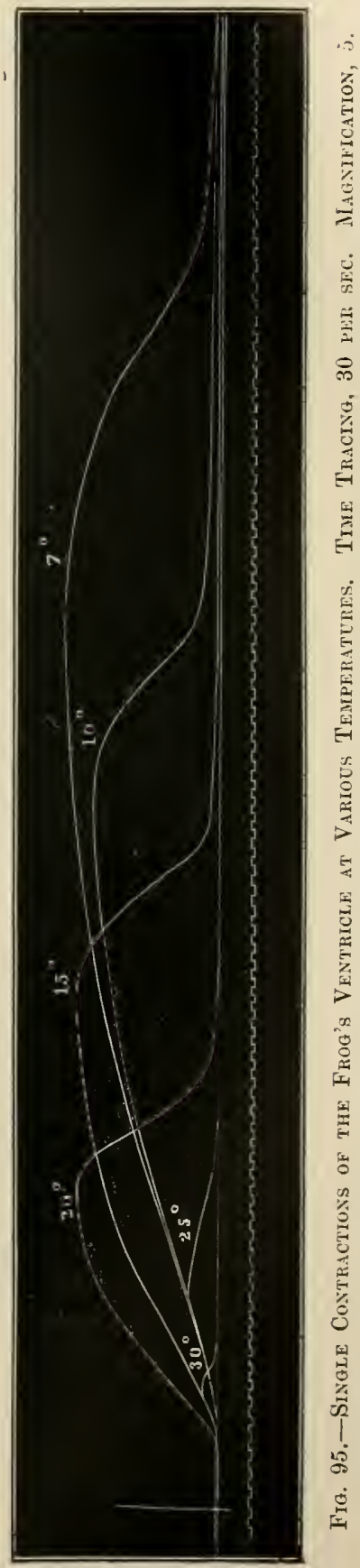




\begin{tabular}{|c|c|c|c|c|c|}
\hline \multirow{2}{*}{ Temperature } & \multicolumn{3}{|c|}{ Duration in $\frac{1}{30}$ th Sec. of } & \multirow{2}{*}{$\begin{array}{c}\text { Total } \\
\text { Time of } \\
\text { Contraction }\end{array}$} & \multirow{2}{*}{$\begin{array}{l}\text { Height of } \\
\text { Contraction } \\
\text { in } \mathrm{Mm} \text {. }\end{array}$} \\
\hline & $\begin{array}{l}\text { Latent } \\
\text { Period }\end{array}$ & $\begin{array}{c}\text { Period of } \\
\text { Contraction }\end{array}$ & $\begin{array}{c}\text { Period of } \\
\text { Relaxatiols }\end{array}$ & & \\
\hline $7^{\circ} \mathrm{C}$ & $4 \cdot 5$ & 49 & $28 \cdot 5$ & 82 & 17 \\
\hline 10 & 4 & 39 & 11 & 54 & 13.5 \\
\hline 15 & 4 & 27 & 12 & 43 & 16 \\
\hline 20 & 3 & 14 & 12 & 29 & 16 \\
\hline 25 & 3 & 9 & 11 & 23 & 3 \\
\hline 30 & 3 & 4 & 4 & 11 & 1.5 \\
\hline
\end{tabular}

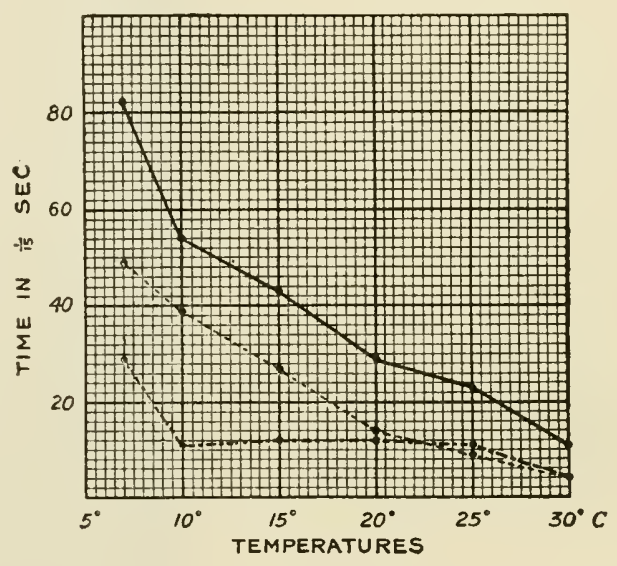

FIG. 96.

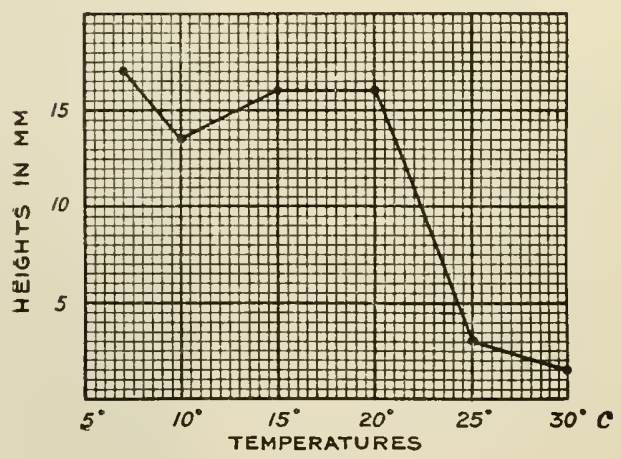

FIG. 97.

then remained perfectly constant until $30^{\circ} \mathrm{C}$. was reached, when there was again a sudden diminution. 
4. The total time showed at first a rapid diminution, and then a more gradual one as the temperature rose.

5. The height of contraction showed a maximum at $7^{\circ} \mathrm{C}$., a relative minimum at $10^{\circ} \mathrm{C}$., a relative maximum from 15 to $20^{\circ} \mathrm{C}$, and an absolute minimum at $30^{\circ} \mathrm{C}$.

These different points can be very clearly figured by the use of squared paper, and should be carried out as in figs. 96 and 97.

These conclusions should also be compared with those we have already obtained when studying the effect of varying the temperature upon striated muscle (p. 46). The results are found to very closely resemble each other in most of their important features. 


\section{CHAPTER XI}

THE NERVES OF THE FROG'S HEART AND THEIR FUNCTIONS

MAKE A DISSECTION TO SHOW THE NERVES OF THE FROG'S HEART. - The nerve supply to the frog's heart is from two sources, the vagus and the sympathetic.

To find the vagus expose the heart in the usual manner, leaving, however, the pericardium intact to serve as a protection while the dissection is being made. Pull aside the lateral pieces of the sternum and separate the muscles running from it to the floor of the mouth. When this is done a muscle comes into view which is the guiding mark for the vagus. This muscle is the petro-hyoid, which arises from

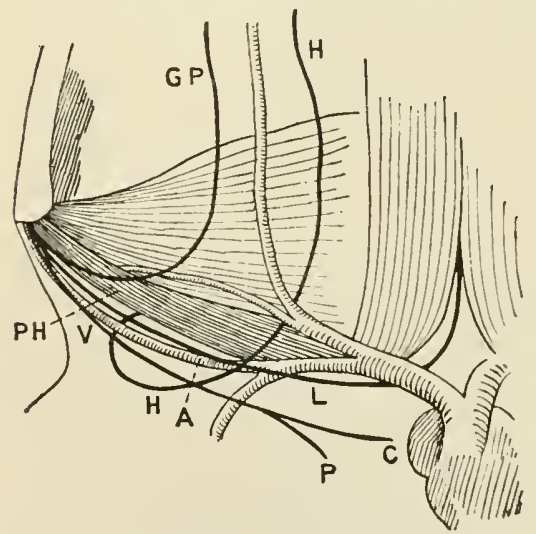

Fig. 98.-To Show the Course of the Vagus in the Frog. the base of the skull and is inserted into the posterior cornu of the hyoid bone. Its direction is from the joint of the lower jaw, round the pharynx in an almost transverse direction to the body of the hyoid. This muscle should be clearly made out (fig. 98 , $\mathrm{PH})$. It is arranged in three separate small bands, in series one behind the other. Crossing it are two nerves: one laterally placed, the glosso-pharyngeal, GP, coursing from the angle of the jaw over the muscle to run forwards into the tissues forming the floor of the mouth; the other, the hypoglossal, $\mathrm{H}$, crosses it much nearer the mid line. The latter nerve is the first spinal nerve in the frog. In relation to the lower border of the muscle lies the carotid artery, $A$. If the muscle be now laid hold of, a nerve will be seen running along its lower edge and partly covered by it. This is the vagus, v. A branch of this, the laryngeal, L, usually runs a separate course parallel to the main trunk, 
leaving it as the nerve comes towards the anterior mid line. The main trunk of the nerve branches near the heart, the larger half running backwards towards the lung; the other is the cardiac branch. The vagus must be isolated completely from the surrounding tissues. It is important to remember that the nerve thus isolated is not the vagus only, but has already been joined, immediately after its exit from the skull, by a large branch from the sympathetic. The cardiac branch therefore contains both vagal and sympathetic fibres.

To expose the sympathetic before it joins the vagus, cut away the whole of the lower jaw by a single transverse incision. Pick up the mucous membrane covering the roof of the mouth and cut it away, removing it well down to the asophagus. This brings into view a triangular-shaped muscle, the levator anguli scapulæ, M, fig. 99. Very carefully cut this through near to its attachment to the base of the skull and turn it down. This brings the sympathetic into view. It is usually accompanied by a bloodvessel which lies over it, and being pigmented serves as a guide to the nerve.

The nerve is very readily seen as it crosses the large root of the second nerve, above which it bifurcates to pass round the subclavian artery, forming the annulus of Vieussens. Carefully isolate the sympathetic between the annulus and its junction with the vagal trunk. Pass a fine ligature round it and tie it as far down as possible.

Cut the nerve below the ligature. The cardiac fibres of the sympathetic leave the cord mainly in the ramus communicans from the third nerve, and to a less extent in that of the fourth nerve. In the same dissection

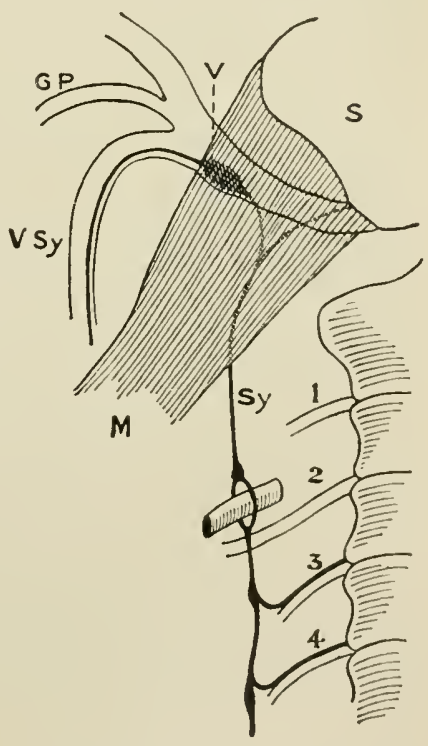

| Fig. 99.-The Course of the Srmpathetic in the Frog. it is easy to expose the petro-hyoid muscles and remove them, and in this way the vago-sympathetic in its course from the ganglion of the trunk of the vagus to the heart can be readily traced.

Having exposed the two vagi and passed threads under them their action should be studied as follows:- 
Experiment 1.-Study the Influence of the vagus upon the heart. Prepare the coil for giving tetanising shocks. Dissect out both vagi. It is best to cut the laryngeal branch of each vagus because if this be left intact the muscles contract when the nerve is stimulated and acting upon the pericardium, pull down the base of the heart, and so alter the level of the tracing. Attach the heart to the recording lever by the suspension method, fig. 83. If the heart be beating very slowly apply a little warm normal saline solution (at $20^{\circ} \mathrm{C}$.) by a pipette until the beat is quickened. Remove the secondary coil to some distance from the primary. The electrodes, fig. 87, should have their ends bent up into a hook, and the outer sides of the wires may be further insulated by a little sealing-wax to prevent escape of the current. Hook up one vagus on to the electrodes. Now take a piece of tracing, the drum rotating about 1 to $2 \mathrm{~mm}$. per second, and with a seconds time tracing recorded directly beneath. After about ten contractions open the key in the secondary circuit, so that the nerve may be stimulated, and mark on the tracing the instant at which the stimulus was applied. If no change occur close the key, marking the instant at which the stimulation was stopped. Next move the coil $2 \mathrm{~cm}$. nearer the primary, and repeat the stimulation as before. Repeat several times, gradually increasing the strength of the stimulus until one or two tracings showing complete inhibition have been recorded. Time must be allowed after stimulation is stopped to record the full series of changes occurring after stimulation. Repeat the series, stimulating the vagus of the opposite side.

It must be remembered that in this experiment we are stimulating both sets of cardiac fibres, sympathetic as well as vagal.

In fig. 100 the result is shown. The laryngeal branch was not cut, and we see that on each stimulation the general level of the record was lowered. When the secondary was at $19 \mathrm{~cm}$. (tracing 1) there was a very slight slowing effect. One evidence of this is to be seen in the auricular tracing. Before stimulation the auricle began to contract before relaxation of the ventricle was complete. During the stimulation, however, the time interval between two successive beats is very slightly increased, and this allowed relaxation of the ventricle to be completed before the next auricular contraction commenced. In tracing 2 with an increase in strength of stimulation there is a clear diminution of rate. Note that the effect does not occur instantaneously. There is a gradual production of the slowing lasting over four beats. The rate before stimulation was 38 per minute; towards the end of the time of stimulation it was 30 per minute. A second change, occurring as a result of the stimulation, is an increase in the force of each beat as judged by the height of the tracing. In the measurement of these tracings, where we are examining the heights of contraction, it must be remembered to measure from the point where the ventricular contraction begins to the highest point reached. It will not do to take the total height of the tracing as the measure of force, because at times a considerable amount of the rise may be due to auricular contraction with ventricular superadded; at other parts of the tracing it may be purely ventricular contraction. Examine in this connection fig. 102 
In the piece before stimulation this is $9 \mathrm{~mm}$, during stimulation it is $11 \mathrm{~mm}$., and after stimulation it soon returns to its initial height. A third change to be observed is with regard to an altered rate of transmission of the contraction from the auricle to the ventricle. Before stimulation the auricular beat was practically completed before the ventricular began. Under the influence of the stimulation the time

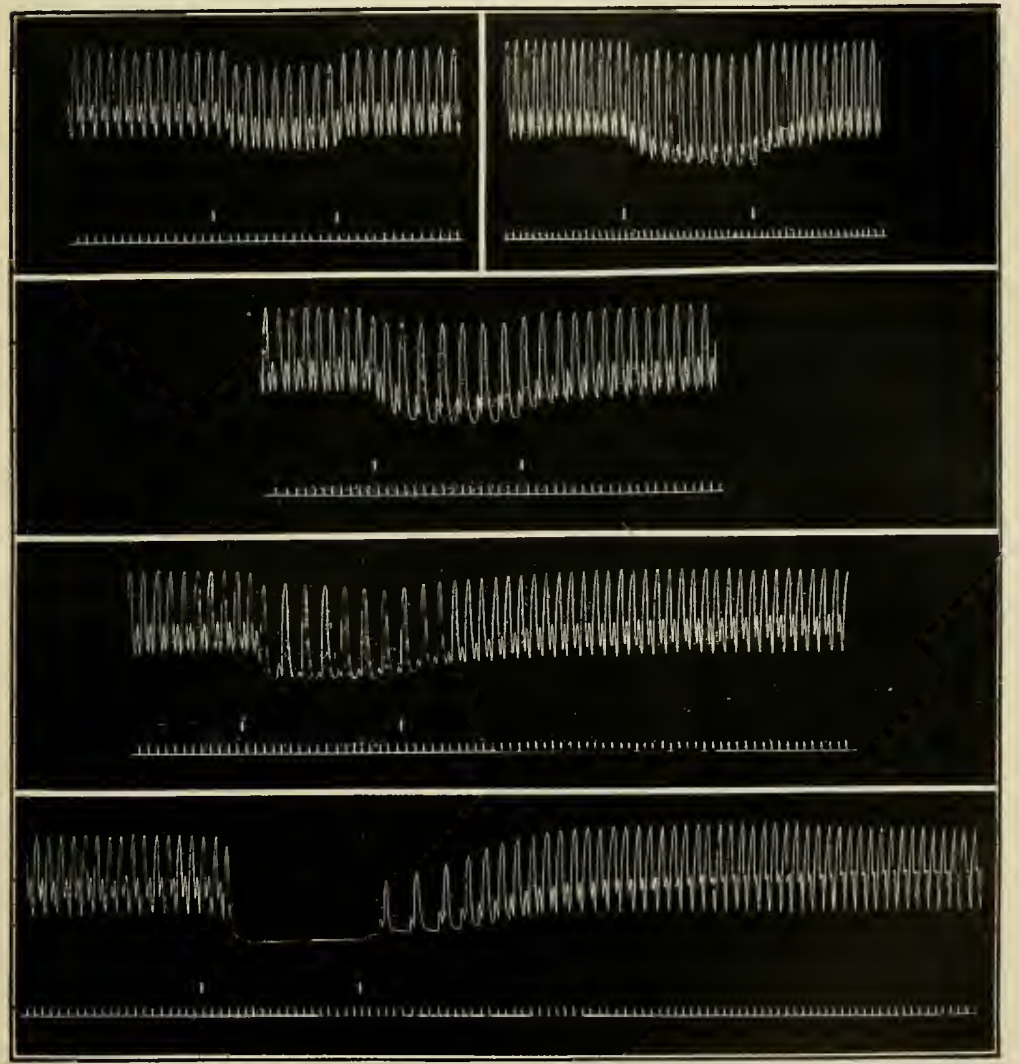

Fig. 100.-Effects of Tetanising the Vagus with Different Strengths of Striuli. In Tracing 1 the Secondary Coll was at 19 chs.; in 2 at 18 cis.; in 3 at 16 cMs.; in 4 at 15 cMs.; and in 5 at 14 cMs. Fron the Primary Coil. Time Tracing Seconds.

relation gradually changed and showed that the wave of contraction passed more readily from auricle to ventricular, i.e. that conduction between the two was favoured.

In tracing 3 , with slightly increased strength of stimulus, slowing is more pronounced, from 35 to 25 per minute. The height of each is 
again increased $-8 \mathrm{~mm}$. before as compared to 10 during stimulation. There is again an increase in the rate of conduction of the contraction wave from auricle to ventricle. There is also less delay between the commencement of stimulation and the production of its effect than in the preceding case.

In tracing 4 the same points are again to be observed, but it is noticeable that the slowing becomes less marked as stimulation proceeds. Rate of beating was 36 before and 24 during stimulation; the height before, $8 \mathrm{~mm}$; during stimulation, $9 \mathrm{~mm}$.

In tracing 5 we see that after two beats the heart comes completely to rest. There is a delay before the effect of the stimulus is apparent. After stimulation ceased a short pause occurred, and then the heart recommenced to beat. The rate gradually increased. Thus the time interval between the commencement of the second beat and corresponding point of the first is $3 \frac{1}{2}$ secs. ; between 3rd and 2nd, 3 secs.; between 4 th and $3 \mathrm{rd}, 2 \frac{1}{2}$ secs. It then quickly regained its original rate of 43 per minute. The height of the beat also shows a gradual increase. The measurements are: For the 1 st, $4 \frac{1}{2} \mathrm{~mm}$; the 2nd, $5 \frac{1}{2} \mathrm{~mm}$.; the $3 \mathrm{rd}, 6 \mathrm{~mm}$. ; the $4 \mathrm{th}, 6 \frac{1}{2} \mathrm{~mm}$; the $5 \mathrm{th}, 7 \mathrm{~mm}$; and then in a few beats it attained a height of $9 \mathrm{~mm}$. as compared to one of $8 \mathrm{~mm}$. before stimulation. Thus inhibition has had a beneficial effect upon the ventricle, enabling it to beat a little more forcibly for a time, but this gradually dies away, and in about 20 beats the height is once more $8 \mathrm{~mm}$. The same holds true even to a more marked degree for the auricular contraction. The rhythm between auricle and ventricle beats also shows a very interesting change. In the first beat after the stimulation the ventricular contraction commences shortly after the commencement of the auricular relaxation; in the second beat at a rather later time; and as the beats follow one another the ventricular beat gradually falls later in the auricular diastole. Associated with the gradual slowing of the ventricular beat with respect to the auricular it is seen that the auricular contraction begins progressively earlier with respect to the ventricular diastole, and that at last it occurs when about one half of the diastole is completed. As this happens the auricular systole becomes progressively less and less marked, and finally is only represented as a break in the descent representing the ventricular systole. These facts tend to show that conduction of the contraction wave from auricle to ventricle is at first rapid, but gradually becomes slower than normal as an after-effect of the vagus inhibition, once more running to its normal rate as that after-effect gradually wears off.

We must remember that in the results we have just been examining we are not dealing with the result of stimulation of pure vagal fibres, 
but with that of the mixed vagus and sympathetic. To determine which of these results are due to vagal fibres only, our only procedure can be to stimulate the vagus inside the skull before it has been joined by the sympathetic fibres. We may state as the general result of impulses passing down the vagus fibres-

(i.) That they cause slowing or inhibition of the beat, depending upon the strength of stimulus employed.

(ii.) That they tend to weaken the force of the beat when slowing occurs.

(iii.) That as an after-effect there is for a time an acceleration accompanied by au augmentation of the force.

There is often to be observed a difference in effect between the two vagi. Sometimes one vagus is found not to possess any inhibitory fibres, in which case the opposite vagus is found especially active. It is usually found that the effect is not identical on the two sides, one usually being more powerful than the other.

Experiment 2.-Contrast the effect of stimulation of the crescent with stimulation of the vagus. Prepare the apparatus as for the preceding experiment. Dissect out both vagi, passing fine threads round them, so that they may be readily picked up when required. Having attached the heart to the lever place one vagus upon the electrodes, and after recording a short piece of normal tracing send in a tetanising current into the vagus and record its effect. Next repeat on the vagus of the opposite side. Finally apply the electrodes, so that they touch the crescentic junction between sinus and auricles, and record the result of stimulation in this position.

Fig. 101 gives the result of such an experiment. The first tracing is interesting because no inhibition resulted on stimulating the right vagus. The only alteration seen is a slight change in the sequence of the beat, the ventricular contraction commencing a little earlier in the auricular relaxation. Stimulation with stronger currents also had no inhibitory effect. Stimulation of the left vagus produced a typical inhibition (II, fig. 101). Finally in III is seen the effect of stimulation of the crescent. In this latter there are but slight differences from a typical vagus effect. Complete inhibition follows after a short latency, and on cessation of stimulation there is after a short latency a return of the beat, which shows the staircase effect, though not so clearly as in II. After the stimulation the auricular contraction tends to commence earlier in the ventricular relaxation, a change gradually occurring, until finally it is seen to begin at about the middle of the relaxation.

Experiment 3.-Examine the action of the sympathetic upon the heart. Arrange the apparatus as in the previous experiment. Dissect out the sympathetic on one side, placing a ligature around it, as described on p. 121. 
Arrange the heart to record as before, and place the nerve upon a pair of electrodes. Carry out the experiment upon a similar plan to that used for the vagus, and so obtain a series of tracings showing the effect upon the heart with different strengths of stimulation. If the heart be beating rapidly the

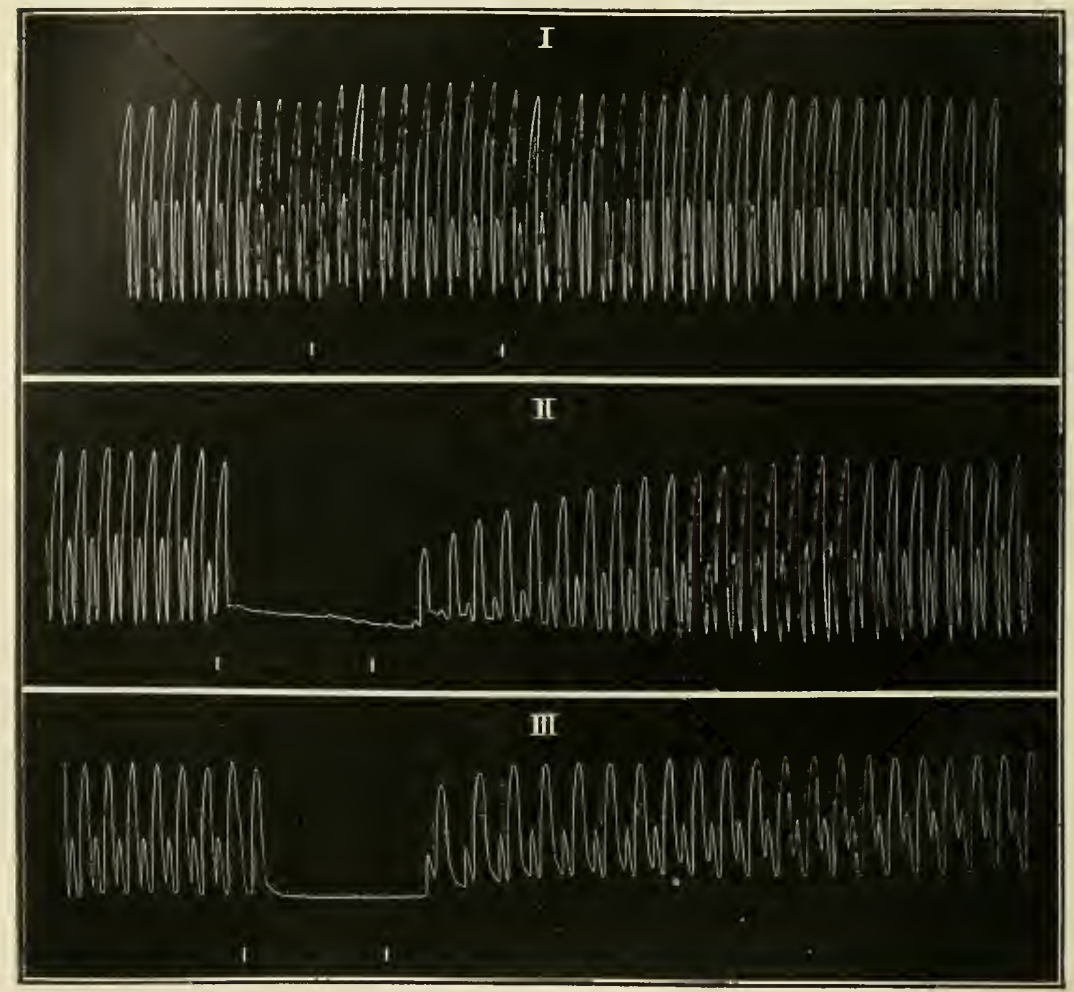

Fig. 101.-Tracing i Shows the Effect of Tetanisation of the R. Vagus ; il of the L. Vagus; and iI of the Crescent. In all Cases the Sajie Strength of Stinulus.

influence of the stimulation is not very marked, in which case the heart should be cooled by allowing a little normal saline, which has been cooled by ice, to drop on to it until its rate is slowed.

Fig. 102 shows a typical result of such an experiment: the upper two tracings are from the same heart with different strengths of current, the third from a different heart. The first shows that during stimulation the following changes occur:-

i. Acceleration of the beat. Before stimulation this was 15 per minute, during stimulation 30 per minute. After stimulation ceased the heart gradually slowed again until its initial rate was once more reached. 
ii. An increase in force. Before stimulation the height of the ventricular beat was $9 \mathrm{~cm}$., but during stimulation it reached $12 \mathrm{~cm}$. These measurements must be made from the lowest to highest points
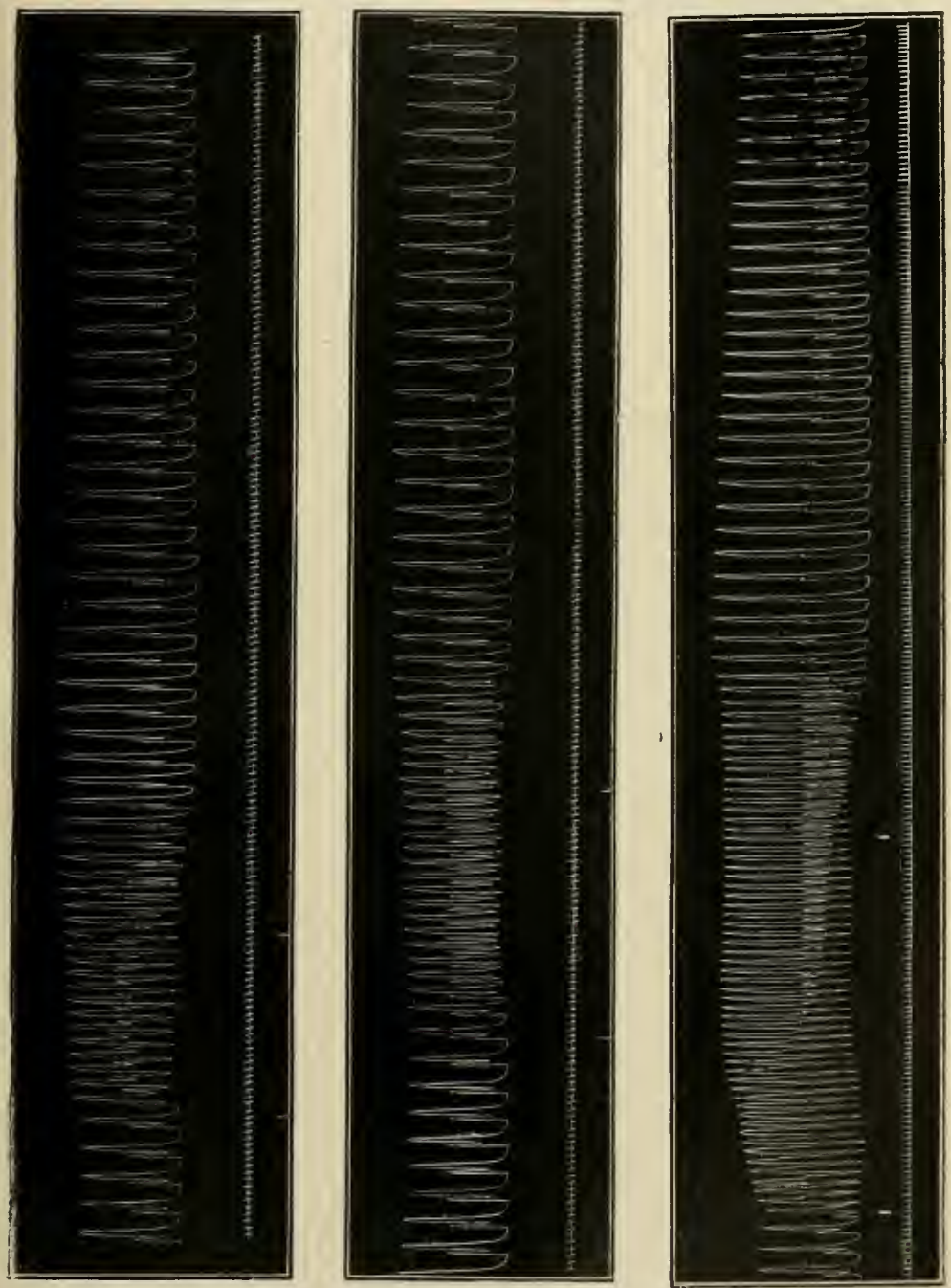

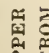

का है

国

Ho

군

菓藏

舟

볼

ठึ

元

幽

ชั

密

น

禹 $\infty$

P

둔

도

氙帚

of

㠃㕕

…

일

थै ठิ

淟皆

ए

긍봉격

我

邑

글 운

它

180

จंष्य कि

$+-1$

氙是

点

of the ventricular contraction, for the increase in force is to a considerable extent masked by the change in character of the trace brought about by the altered sequence. An increase in force of auri. cular beat is also produced, though to a less relative degree than with the ventricular.

iii. An alteration in the sequence. There is produced a delay in 
the conduction of the contraction from auricle to ventricle, so that the auricle has quite relaxed before the ventricular systole begins. Further, as the rate of beating is faster, it is seen that a contraction of the auricle commences before the ventricular contraction is complete.

iv. A gradual return to the normal state after stimulation has ceased. The acceleration gradually disappears. The force of the beat at once begins to decline until it reaches the original amount. A change in conduction occurs in the reverse direction to that found during stimulation. Rate of conduction is increased, and ventricular systole commences soon after the auricular contraction reaches its maximum. The result is that there is more summation of auricular contraction to ventricular, with the result that the total amplitude of the trace is greater than during stimulation, though each individually is less. This increased rate of conduction gradually dies away and returns to the original value.

These results should be directly contrasted with those found on vagal stimulation (p. 122), where they are seen to be in exactly the reverse direction.

In II a similar effect is found in all particulars, differences being only of degree. Rate is increased from 16 to 32 ; height from $9 \mathrm{~mm}$. to $11.5 \mathrm{~mm}$.; and change of sequence is of the same character.

Tracing II is from a less exhausted heart, and shows the changes very clearly.

Rate before stimulation was 25 , during stimulation 42 per minute.

Height of ventricular contraction before stimulation was $9 \cdot 5$, during stimulation $14 \mathrm{~mm}$.

Change of sequence is at first as in previous tracings, but this is then followed by another alteration in which the auricular contraction commences later in the ventricular relaxation, though conduction is still delayed as previously. After stimulation ceases the same stages are passed through as were previously described, and to a very marked degree.

The Second Stannius Ligature.-In a previous series of experiments we have seen that a ligature applied between the sinus and auricles leads to a standstill of the auricles and ventricle in diastole, while the sinus continues beating with unaltered rhythm.

Experiment 4.-Complete the Stannius experiment.by applying a second ligature at the junction of the auricles with the ventricle.

It will usually be found that the auricle still remains quiescent, but that the ventricle begins to beat. Count the rate at which sinus and ventricle beat. It will be found that the ventricle is beating at a slower rate than the sinus. This is the rule, but occasionally it is found that the auricle only begins to beat, or again that both start beating. As to the meaning to be given to this 
experiment, opinions differ. One view is that the first ligature causes standstill of auricles and ventricle by stimulating the inhibitory mechanism at the junction of sinus and auricles, and that this inhibition lasts some time, until the mechanism becoming fatigued, the inhibited parts gradually regain a rhythmic contraction. This view is borne out by repeating the experiment upon a heart poisoned by atropine. The atropine paralyses the inhibitory nerve terminals of the heart, and it is found that standstill is impeded or prevented by atropine. The second ligature is supposed to act by cutting off the inhibitory influences, set in action by the first ligature, from the ventricle, which then commences to beat at its own rhythm.

According to Gaskell, the probable explanation of the experiment is that the first ligature blocks the contraction wave originating in the sinus. Auricles and ventricle, therefore, for a time remain quiescent, ultimately originating a rhythm of their own, though this requires time. This does not, however, thoroughly explain all the facts, for if the auricles and ventricle be excised, they gain a fresh rhythm in quite a short time, and it therefore seems to follow that the first ligature must be causing some inhibitory influences preventing the establishment of that rhythm. On Gaskell's view the action of the second ligature is to stimulate the ventricle, and consequently to lead to a rhythmic contraction. It is difficult to see why the first ligature should not also act as a stimulus, or why the second should not stimulate the auricle rather than the ventricle. 


\section{ACTION OF MUSCARINE AND ATROPINE}

Experiment 1.-Take a piece of fairly wide glass tubing and draw it ont at one end to form a pipette with very fine orifice. Arrange the apparatus for recording the heart beats by the suspension method, having dissected ont the vagi. Record the effect of stimulating the ragus and the sinus. Take some of the muscarine solution ${ }^{1}$ in the pipette and allow it to fall, drop by drop, on to the heart while it is still recording. Almost at once the beat becomes slower, and gradually force and rate decrease until the heart comes to complete standstill in diastole. Stimulate the heart, either mechanically or electrically. It will be found to require a very strong stimulus to make it respond. After allowing it to remain at rest for a short time to see that there is no tendency to recovery, wash out the pipette and fill it with the atropine solution. Let the solution fall on to the heart. Gradually the heart begins to beat again, and shows precisely the same phenomena as after inhibition by vagal stimulation. If the heart had been beating weakly before the application of the muscarine solution, its beats after the application of the atropine often attain a much greater amplitude.

Next place one of the vagi on the electrodes and tetanise it. No slowing nor inhibition occurs. Next apply the electrodes to the crescent; still no inhibition takes place. The atropine has paralysed the inhibitory nerve terminals in the heart substance. The fact that atropine abolishes the muscarine effect proves that muscarine also acts on the nerve mechanism, and not directly upon the heart muscle.

In fig. 103 a record taken during such an experiment is given. The solution of muscarine was applied after the fifth beat of tracing I, and very quickly a change in the rhythm of each beat was produced. The auricular beat, which previously commenced during the ventricular diastole, was delayed and became less forcible. The sinus contraction also became marked on the tracing. Gradually the force of the auricular beat became less and less, though for a time that of the ventricular beat was maintained. Later, the ventricular contraction became less forcible and slower, and finally suddenly ceased. The line seen at the end of the tracing shows undulations which were due to the sinus beat.

\footnotetext{
1 Made by adding a drop of a strong muscarine solution to some normal saline solution.

"A $\frac{i}{2}$ per cent. solution of the sulphate in normal saline.
} 
After about a minute had elapsed atropine solution was dropped on to the heart, commencing at II, fig. 103. After an interval a small contraction of the ventricle occurred, and gradually this increased in force and frequency until the character of the beat was once more regained. It is also seen that the force of the auricular beat was regained, though more slowly than the ventricular. In about the middle of this tracing the rate of conduction of the contraction wave from auricle to ventricle is seen to be slow, and from that time on to increase, until finally it became very rapid. Atropine, then, favours the conduction of the contraction from auricle to ventricle. Tracing III shows that stimulation of the left vagus with strong tetanising shocks thrown in from $a$ to $b$ produced no inhibition nor change of force. In Iv the sinus was stimulated. This also proved ineffectual, though, previously, stimulation of both vagus and sinus produced the usual inhibi-

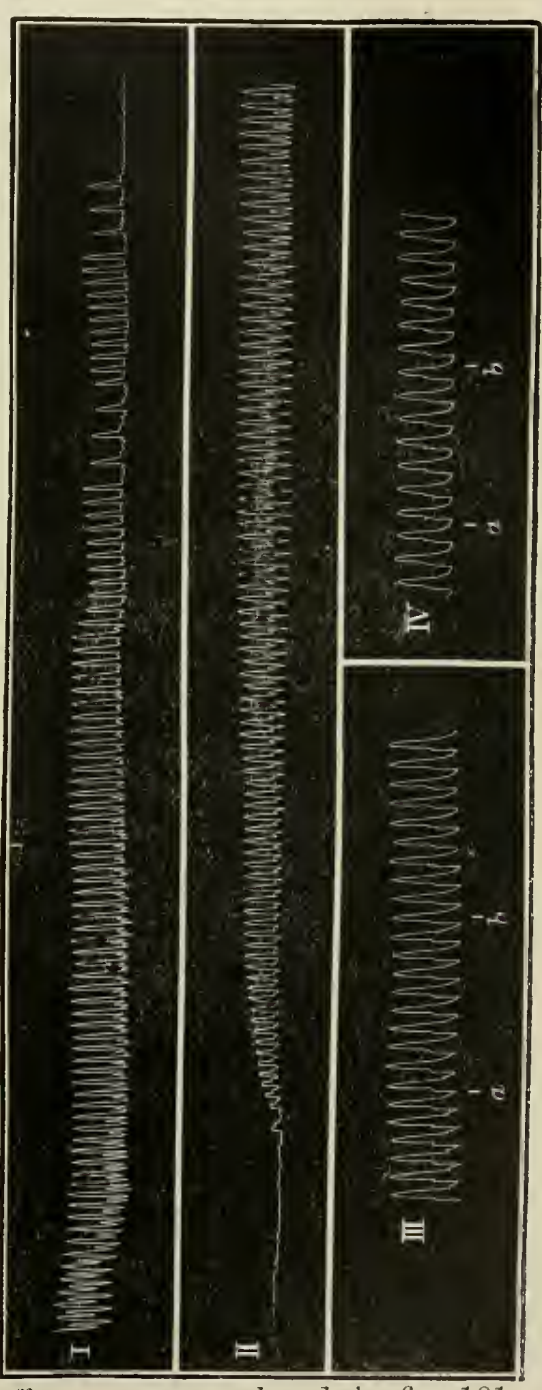

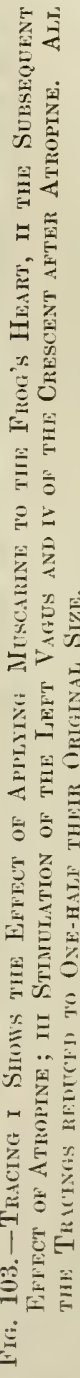
tion. These inhibitory effects are reproduced in fig. 101, p. 126. taken just before muscarine had been applied.

Experiment 2.--To a fresh heart arranged as in the previous experiment apply a few drops of a $\frac{1}{2}$ per cent. solution of pilocarpine nitrate. It acts similarly to muscarine, and its effect is abolished by atropine. 


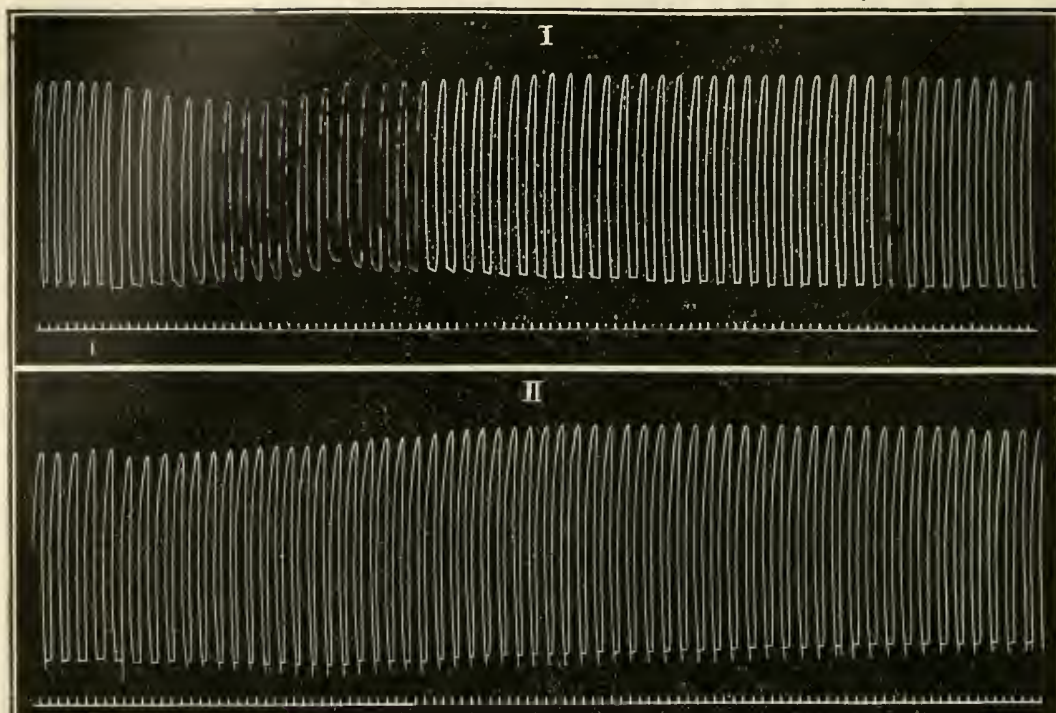

1

1
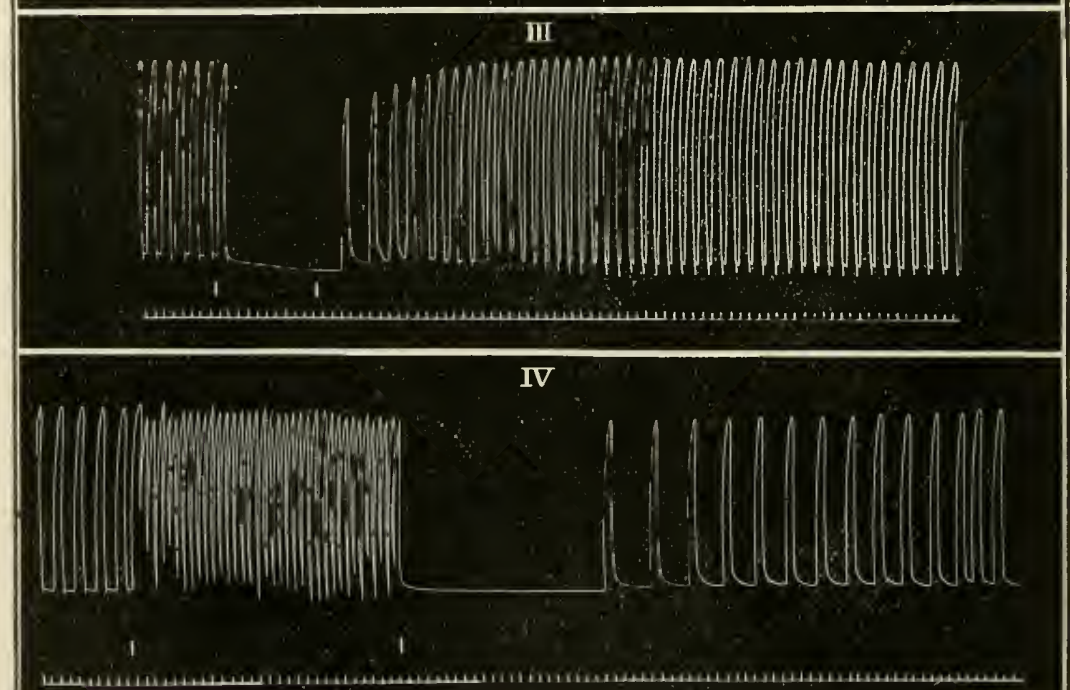

Fig, 104.-Tracing i Shows the Effect of Applyivg a Weak Soldtion of Nicotine Directly to the Heart. The Short Vertical Mark Inuicates the Instant at which it was applied ; il Gives the Result of Stmullation of the Vagts ; in of Stimulation of the Crescent ; and iv of Stinulation of the Crescent aftel a Ftrther Dose of Nicotine had been Aprited. Tine Tracing Secunis. Magnification, 5. 


\section{ACTION OF NICOTINE}

Experiment 3.-Arrange a heart to record as before, having previously isolated one vagus and placed it upon a pair of electrodes. Test the vagus to see that it causes inhibition on stimulation. Record a few normal beats and then apply a few drops of a 0.1 per cent. solution of nicotine in normal saline. The heart is slowed for a few beats and then beats rather quicker than before. Now stimulate the vagus. There is no inhibition. Apply the electrodes to the crescent. The heart is inhibited.

The action of the drug in a weak solution is to first stimulate nerve cells and then to paralyse them. The stimulation is shown in the inhibition, which may be but slight or fairly well marked, according to the strength of the solution. After a few beats the heart regains its rate, and may even become quicker and the force greater than before (I, fig. 104). In II is shown the effect of stimulation of the vagus. No inhibition or slowing follows even with strong stimuli, though previously the inhibitory effect had been very readily produced. During the stimulation an augmentor effect is produced, the height of the beat becoming $23.5 \mathrm{~mm}$. as compared with $21 \mathrm{~mm}$. There is only slight acceleration, and both effects gradually die away. In III is seen the effect of stimulation of the sinus. It is perfectly characteristic of the result given by a normal heart (fig. 101, III). All these three tracings were taken quickly one after the other, and then more nicotine solution was applied. It was then found that stimulation at the sinus no longer produced inhibition. With weak currents no effect at all was perceived except a slight acceleration. The strength of the stimulus was then considerably increased, when marked acceleration was produced lasting as long as stimulation continued. On cessation of stimulation the heart was inhibited, but after a time recommenced beating with a rhythm at first slow, but gradually increasing until the original rate was once more attained.

This experiment is of great importance because it affords an excellent example of the value of nicotine, as it is now employed for determining the position of nerve cells on the course of visceral nerve fibres. It is found to first stimulate these cells and then paralyse them, and if the dose be increased the nerve fibres themselves also become paralysed. This is a general rule for all visceral nerve fibres and cells, and in the experiment as above carried out proves that cells are interposed on the course of the vagus fibres, and are situated in the region of the crescent; but that on the other hand the sympathetic fibres run straight to their teminals without having nerve cells interposed on their course within the heart. 


\section{CHAPTER XIII}

\section{SONE FURTHER METHODS FOR EXAMINING THE ACTIVITY OF THE FROG'S HEART}

THE student must become familiar with some of the other methods which are employed for recording the heart movements other than the suspension method.

Experiment 1.-Take a record of the beat of the ventricle by means of one of the recording levers represented in fig. 69. The foot of the vertical rod $\mathrm{H}$ is to be arranged to lie on the ventricle, and will thus rise and fall with increase and decrease of thickness of the ventricle.

During diastole the ventricle wall becomes flaccid, and thus under the

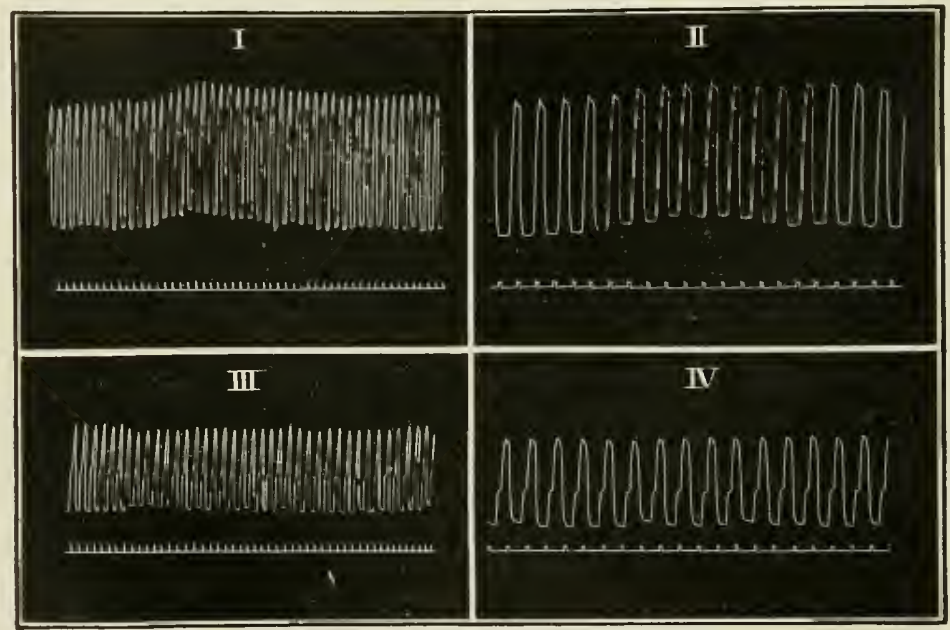

Fig. 105. - Tracixgs Recordei by a Lever Resting tpon a Frog's Heart. Ix Tracings i and it the Lever Rested upon the Ventricle; in III and iv upon the Junction of Auricles witil Ventricle. Tine Tracing Seconds.

influence of gravity acting on its own substance and of the weight of the lever it becomes flattened. With each systole the rentricle hardens and becomes circular in section, and thus lifts the recording lever. If the amplitude of the record be small, it generally means that the pressure of the lever on the heart is too great, and this may be relieved by fixing a wire to the 
lever near the axis and bending it over, so as to lie to the other side of the axis, and thus act as a counterpoise. To its end a small weight can be hung, as, for instance, a piece of folded paper, whose position can be varied until the best amplitude is obtained.

Fig. 105 shows tracings taken by this lever. Tracings I and II are taken with the lever resting on the ventricle only, in II the drum moving rather more than twice as fast as in I. The up-stroke means a contraction of the heart, the down-stroke relaxation. At the end of relaxation there is a pause for a time before a fresh contraction occurs, and in a few of these a slight rise is indicated, which is due to the filling of the ventricle on auricular systole. The contraction is seen to be sustained for a time before relaxation occurs. In tracings III and Iv the lever was placed so as to rest on the junction of auricle and ventricle; we see that the two contractions are now recorded.

Recording by this method we may study any of the results to be obtained in the experiments in which we used the suspension method.

Another method of recording changes in the excised heart's activity is the plethysmographic method.

Experiment 2.-Take a tracing with Roy's tonometer, fig. 106. This consists of a small glass bell-jar whose base fits on a brass support. The joint is made tight by smearing the ground surface of the rim of the glass vessel with lard and rubbing it down tightly on to the brass base. In the

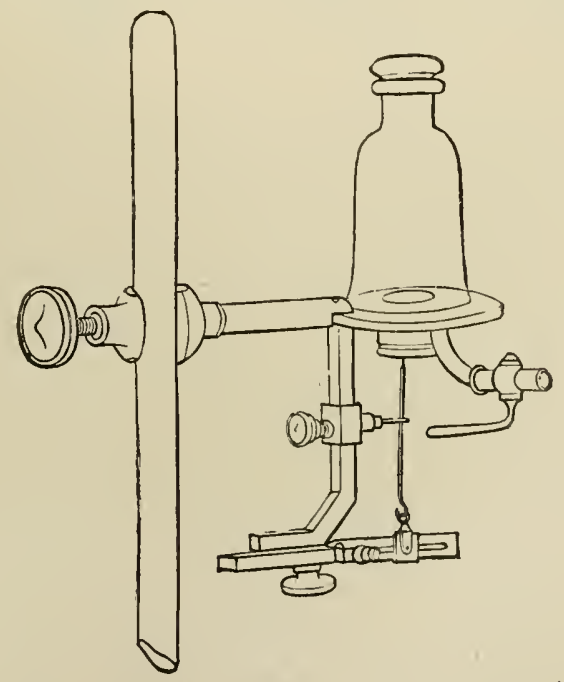

Fig. 106.- Roy's Tonometer. (Hallinurton.)

base is a central hole into which a short cylinder is screwed, and the lower orifice fo this is closed with peritoneal menbrane. A second orifice is fitted 
with a tube, on which is a tap, which is used for filling or emptying the vessel. The heart is tied on to a perfusion cannula fitted in a glass stopper which closes the upper end of the glass vessel. This is then brought into position, and the ressel filled with oil. With each contraction of the heart the volume diminishes and the peritoneal membrane, and with it the recording lever, rises; with each relaxation it falls.

Experiment 3.-Take a tracing with Schäfer's heart plethysmograph. A diagrammatic sketch of this apparatıs is given in fig. 107. The heart is tied on to a two-way cannula and then fitted tightly into a glass bulb filled with oil. On either side of the bulb is a glass tube fitted with a tap. The one to

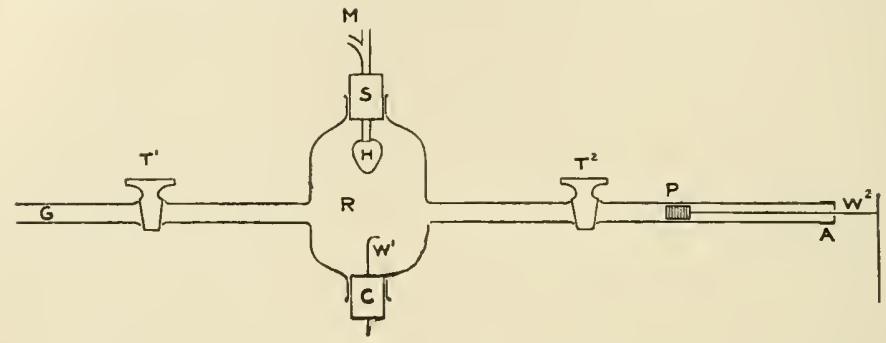

F1g. 107.-Schäfer's Frog-heart Plethysiograph.

the left is for adjusting the amount of oil in the apparatus; that on the right carries a piston recorder bearing a writing point. With each contraction of the heart its volume decreases, and the oil and piston move towards the bulb. The writing point is then caused to record its movements upon a blackened surface.

A simple form may be made and used as in the following experiment :-

Experiment 4.-Take a tracing with the piece of apparatus shown in fig. 108, I. A glass perfusion cannula is made from a glass T-piece, T, one end of which is drawn to a slight constriction, and the end then bevelled off by rubbing it on emery paper and then romnding it in a flame. Through this tube, 0 , a piece of glass tubing, $F$, drawn out to a fine orifice is passed and is cemented in by sealing-wax, c, so that the capillary orifice lies in the orifice of the lower end. This is then fixed in a cork in which a second tube, $A$, is also fixed, and the cork fitted to a short piece of wide glass tubing, $D$. The lower end is closed by a second cork through which a wire, w, passes. Expose a frog's heart and ligature the frenum near the rentricle. Cut the frenum beyond the ligature, lift mp the ventricle, and cut into the sinus transversely. Introduce the camnula, which must previously be filled with diluted ox-blood through this aperture, passing it through the auricle into the ventricle, and tie it in by a ligature passing round the auriculo-ventricular groove. Free the heart from the surrounding parts and wind a fine copper wire round the cannula, touching the ventricle where it is tied to the cannula, so as to form an electrode. Fit the cork into the glass tube, $D$, which is filled with normal saline, so that the fine wire electrode is held tightly between the cork and the glass. Attach the rubber tube on $F$ to a burette containing diluted blood, taking care that no air is included in the tubing. Now record the heart's movements in two ways: (i.) by attaching the tube A to a tambour by tubing of narrow bore. It is best to replace most of the air in this tubing with normal saline, and to interpose on it a glass $T$-piece, 
the lateral orifice being closed with rubber tubing and a spring clip. This gives a record by the plethysmographic method; (ii.) by attaching the tube $\mathrm{T}$ to the end, $\mathrm{A}$, of a small mercury manometer, fig. 108, II. On the tube $\mathrm{A}$ is a three-way tap, $\mathbf{T}$, which can be turned so that the tube $\mathrm{A}$ is connected with the manometer M only, with the exit tube B only, or with both, or completely closed. The movements of the manometer are recorded by a small glass float, F, provided with a glass writing point. 'This latter method is that of Kronecker's frog-heart manometer. The first method records changes in volume. The second method records the pressure attained by the fluid with each contraction.

As soon as the heart is fixed in position it may commence to beat rhythmically, especially if the internal pressure be raised by altering the position

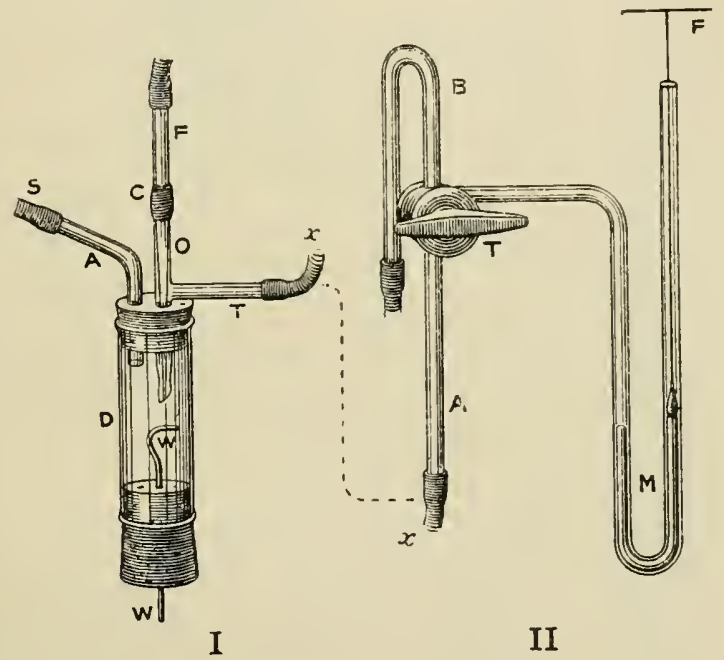

Fig. 108.-Frogi-healit Plethyshograph by which the Pressulie Changes Can also be Recorded by a Simall Manometér.

of the burette of circulating fluid. If it remain quiescent it may be stimulated by sending induced shocks through it by connecting the secondary coil (1) to the wire touching the heart at its base ; and (2) to the stout wire, w. It is not necessary for the upper end of $w$ to touch the heart, it need only be brought near to it. Bring the secondary conl nearer to the primary until the induced current is sufficient to cause a contraction. Repeat the stimuli every three seconds and record the contractions. After a certain time the heart ceases to beat altogether, but before doing so it becomes irregular. A commonly observed form of irregnlarity is where it gives series of beats arranged in groups. These are termed Luciani's groups 


\section{CHAPTER XIV}

DEMONSTRATION OF THE MOVEMENTS OF THE MAMMALIAN HEART.

\section{THE CARDIOGRAPH}

For the experiment a dog, cat, or rabbit is chosen, anæsthetised with ether and morphia, and subsequently curarised by injecting a solution of curare into the external jugular vein. Both vagi are exposed, and ligatures passed round them through an incision in the mid-line of the neck. The trachea is then isolated, and a $\mathbf{Y}$-shaped glass tube tied into its peripheral end. The heart is next exposed by cutting through the sternum with bone forceps, keeping to the midline as far as possible in order to avoid injuring any large blood vessel. The thoracic walls are then drawn well apart, so as to thoroughly expose the heart lying in the pericardium.

As soon as the thorax is opened the lungs collapse and no longer follow the movements of the thoracic wall. The animal would therefore soon die of asphyxia, to prevent which it is necessary to supply it artificially with air. This is done by rhythmically blowing up the lungs through the trachea and then allowing them to collapse.

There are many forms of apparatus which permit of this. A simple but very effective arrangement is shown in fig. 109. A continuous blast of air is obtained by the modified Bunsen pump, P. o is connected to a water tap, and as the water is forced throngh the orifice at $\mathrm{v}$, which nearly fills the constricted neck of the outer receiver, air is drawn in through s. The misture of water and entangled air is collected in the large glass bulb, and the water allowed to flow out through c. The supply of air is directed by a piece of tubing, $A$, to the two-way tap $T$, whence it passes according to the position of the tap along $B$ to $G$, or, as represented in the figure, along the tube $\mathrm{D}$ to a Woulff's bottle, $\mathrm{H}$, containing some ether or other anæsthetic. It then passes from $\mathrm{F}$ to $\mathrm{G}$ and thus to a coil of lead tubing, $\mathrm{k}$, immersed in hot water, so that the air is warmed to body temperature. Another piece of tubing connects it to a $Y$-tube, one end of which, $\mathrm{s}$, is tied into the trachea and the other is covered with rubber tubing which can be partially compressed by the clip I. The blast of air is made intermittent by either fixing a spring clip on $\mathrm{L}$, which is then opened and closed by hand, or the same result is antomatically effected by the arrangement seen at $\mathrm{R}$. This consists of a lever held down by two surings so as to compress the tube against the base-board. The lever is raised intermittently by an eccentric driven from the shafting.

The blast of air is moistened in the pump $P$. The force of distension of 


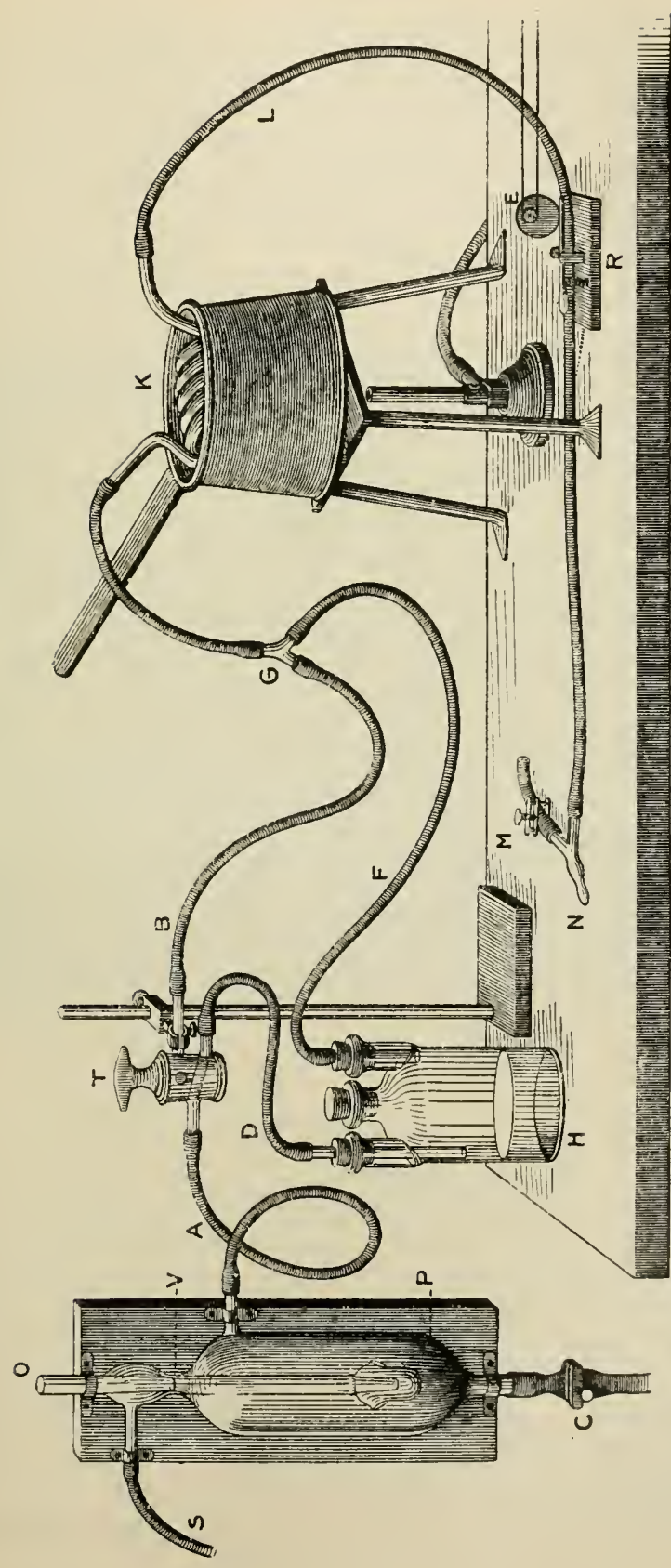

菖

동

본

帘

कठ

留

A 4

$\ddot{1}$

2

닐

울 롭

욘

로․

ำ

叫

B: 워

合

千 品

य

엥

돕

ต

검

E

कi

安

당

步

学

㲾

国息

닌

는

萦

运落

옹

is

D

ำ

준

赔

4

능

政

I :

벽 봉

空

픈

号

定总。

घี 
the lungs can be modified in two ways. First by varying the compression of the tube at $\mathrm{M}$. The increase in resistance at $\mathrm{m}$ raises the pressure at $\mathrm{N}$, and the lungs are therefore more distended. The second method is to vary the pressure of the blast of air. This is attained by compressing c, an increased resistance to the outflow of water leading to a higher pressure of the air. The screw clips are so arranged that sufficient distension is obtained with the lowest pressure of air. Expiration is brought about by the elastic contraction of the lung which drives out the air through the tube $\mathrm{m}$.

The pericardium is now slit up and its cut edges stitched to the thoracic wall on either side. The heart is thus exposed and is attached to the recording apparatus, which consists of two levers, $L^{1}$ and $L^{2}$, fig. 110, which are moved by two fine cotton threads passing over two

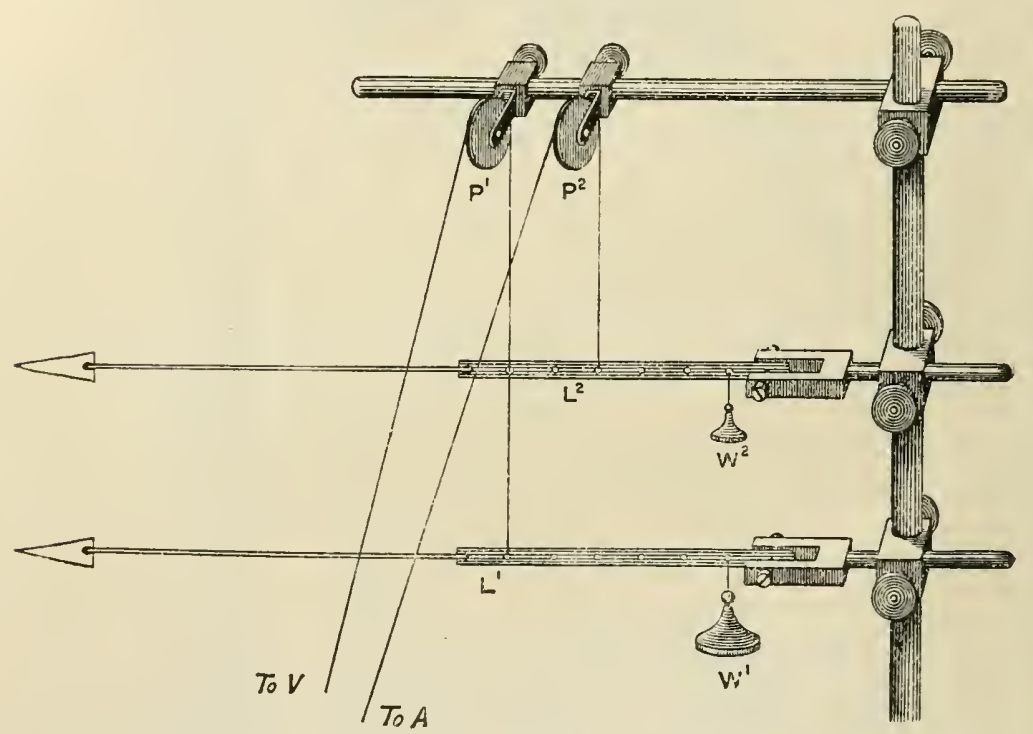

Fig, 110.-Arrangenent of Levers for Reconding the Movements of the Mamialian Heart by Attaching Thleads to the Auricle and Ventricle RESPECTIVELY.

pulleys, $\mathrm{p}^{1}$ and $\mathrm{p}^{2}$. Each thread terminates in a sharp hook, which is passed into a small piece of the ventricular and auricular walls respectively. If the thread from the auricle does not pass freely over the surface of the ventricle it may be made to glide over a glass rod held transversely above the heart, so that the thread is quite free from surrounding parts. The magnification of the lever $L^{1}$ for the ventricle is 3-fold; of the lever $L^{2}$ for the auricle 4 -fold. Each lever is loaded by weights, $w^{1}, w^{2}$, placed near its axis, so as to avoid effects of inertia as far as possible.

The writing levers are brought to the horizontal position by 
adjusting the lengths of the threads and the positions of the pulleys. They are then brought to the writing surface and two chronographs are arranged to write vertically below them-one to give a seconds time tracing, and the other to act as a signal.

Fig. 111 reproduces a piece of tracing obtained in this way, the drum moving at a slow rate. The tracing is from a rabbit's heart, the upper given by the auricle, the lower by the ventricle. It gives a measure of the amount of contraction of the two parts, and indirectly

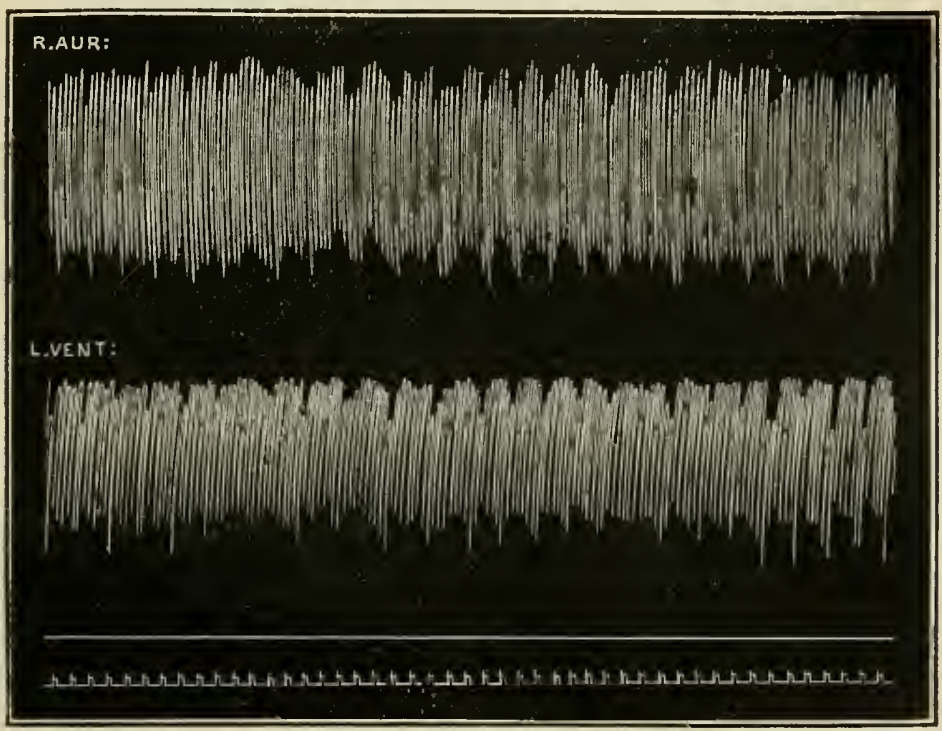

Fig. 111.-Tracing Obtained from the Rabbit's Heart, Employing the Levers of FIG. 110.

of the force of the contraction, the latter especially if the tension of the thread be fairly high. The variations in level of the apices of the ordinates are due to the respiratory movements. Each time the lung is inflated the base of the heart is a little raised, which slackens the thread and the lever descends.

One vagus is now cut and its peripheral end laid upon a pair of electrodes. It is then stimulated a few times and the results recorder. Fig. 112 gives the result of a fairly strong current. The stimulus is seen to act both on the strength and on the frequency of the beat, and to chiefly affect the auricle. Frequency and force are diminished both for auricle and ventricle. After cessation of stimulation the ventricle rapidly regains its previous conclition, but the auricle recovers 
much more slowly; for a short time the extent of its contraction is distinctly less.

The effect of varying the strength of the stimulus is very marked. Weak stimuli primarily affect the auricles, diminishing their rate and

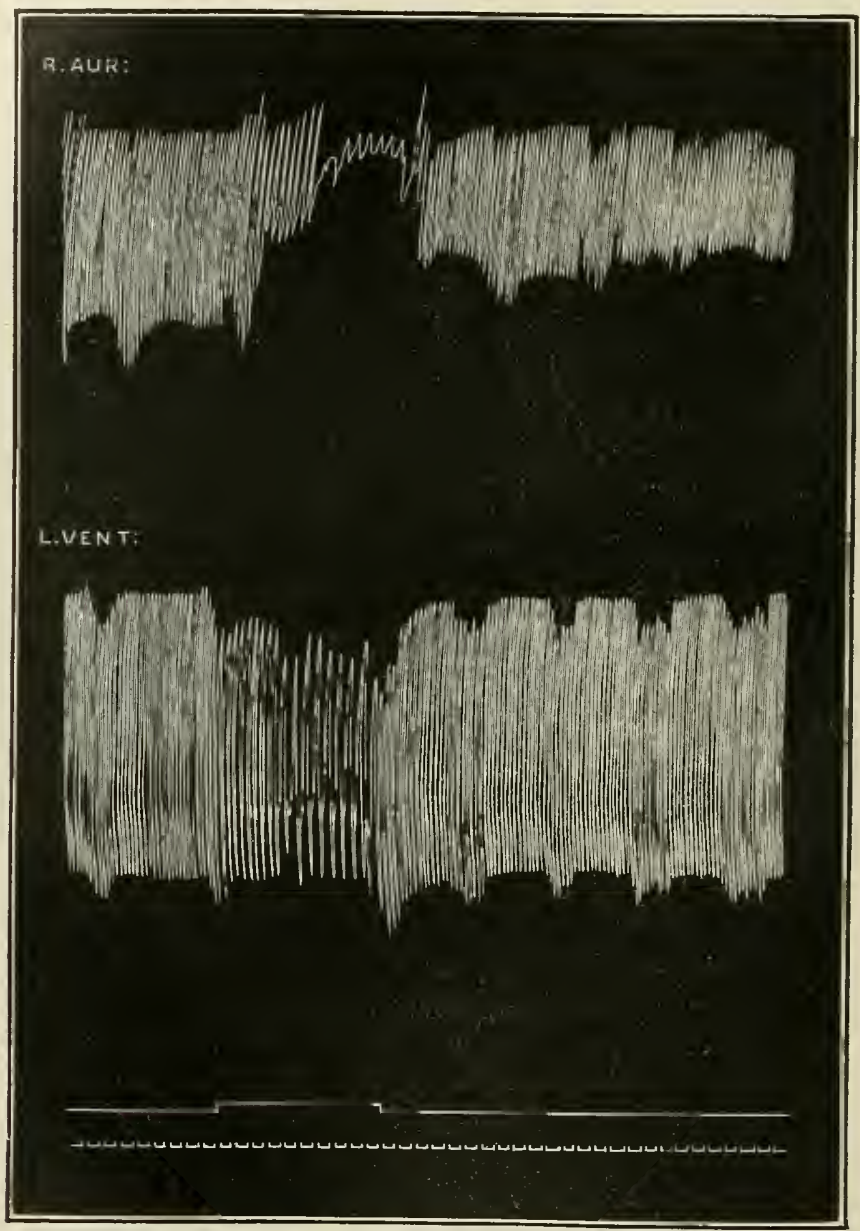

Fig. 112.-Result of the Stimulation of the Left Vagus.

force, but only secondarily affect the rate of the ventricle. Stronger stimuli may inhibit the auricle almost completely, whilst the ventricle still beats with its original rate and force. The strongest stimuli also 
to produce a gradual increase of force of the auricle, and a less marked change in the ventricular contraction when 5 c.c. of a 0.1 per cent. solution are injected. Neurine is found to cause a slight slowing of the beat and a rapid decrease in force of the auricle, soon followed by a gradual increase to about double its initial contraction, and then the effect slowly disappears. Upon the ventricle the changes are in the same direction, though very much less in amount.

To complete the demonstration the animal may be killed by asphyxia. For about the first half-minute the auricular beats increase in amount, remain at this height for some time, and then slowly begin to decrease. The ventricle beats remain practically the same for about $1 \frac{1}{2}$ minute, and then a very rapid change sets in. Some of the ventricle beats are dropped and the auricle beats faster; both decrease greatly in force, and there is at first an increase in general tone, which is rapidly replaced by a decrease in tone, and then the beats gradually decrease and the animal clies. As soon as the rapid change in the heart begins, the right auricle is seen to become more and more filled with blood and soon becomes greatly distended; a condition which persists up to death.

For the purpose of the examination of the heart in man we possess an instrument whose working should be studied in the following experiment :-

Experiment I.-Take a tracing of your orvn heart's impulse by means of the cardiograph (fig. 114). This consists of a tambour, F, the lower end of

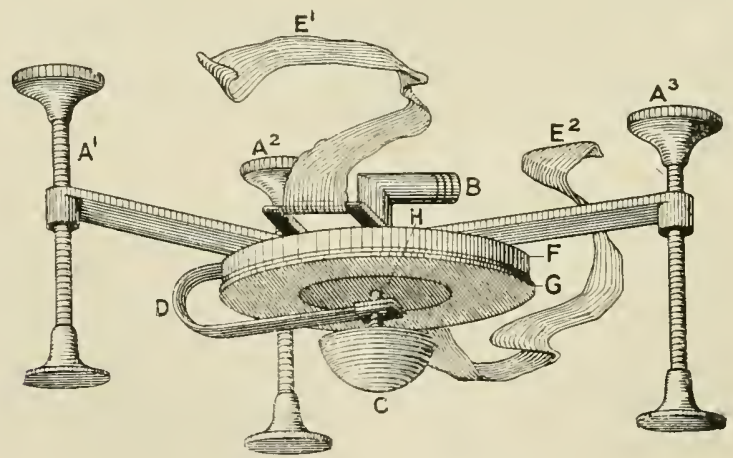

Fig. 114.-The Cardograph.

which is closed by a rubber membrane, $\mathrm{G}$, on the centre of which is cemented a thin aluminium disc, $H$. The cavity of this tambour is placed in communication with a second, the recording tambour, fig. 34, by the tube B. The tambour is fixed on three arms, and at the ends of these are three legs, $A^{1}, A^{2}$, and $A^{x}$, which can be screwed up or down and thus allow a vertical adjustment of 
the tambour. Fixed to the end of a spring D is an ivory button, c, furnished above with a pin which comes into contact with the disc on the tambour. Expose the chest and mark the spot at which the heart's impulse is best felt. Fit the tambour on the chest, so that the tambour rests on the three feet, with the ivory button over the marked spot. It is held in position by the bands $E^{1}$ and $E^{2}$. Now vary the pressure of the button on the skin by altering the position of the three feet until the lever of the recording tambour gives a good excursion. Record a few beats on a drum revolving $\frac{1}{2} \mathrm{~cm}$. per second, taking a seconds time tracing nuder the record.

The form of curve so obtained is shown in fig. 115. It is termed a cardiogram. The moment of hardening is indicated by a sudden

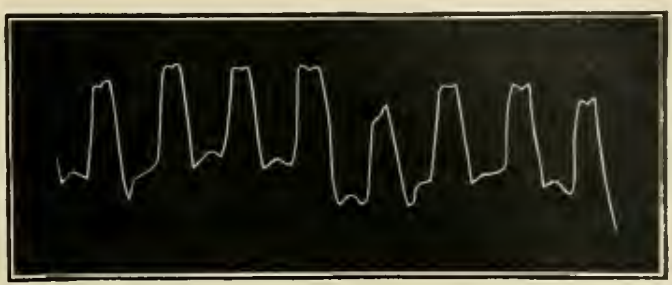

Fig. 115.-A Cardiogrant taken upon a Man. The Contraction of the Heart is recorded by the Up-stroke of the Record.

ascent of the lever and the end of the ventricular systole by a sudden fall of the lever. The chief value of such a tracing is to give us reliable information as to the time at which the heart contracts and relaxes when we wish to compare it with other phenomena occurring at the same time. 


\section{CHAPTER XV}

\section{SOME EXPERIMENTS IN ELECTRO-PHYSIOLOGY}

ThAт animal tissues are of themselves capable of producing currents of electricity was first positively proved by Galvani's experiment of contraction without metals, but our earliest accurate knowledge relating to these currents is due to Du Bois-Reymond, who first gave us measurements of these currents, and taught us how best to study them under different conditions.

If a frog's muscle be excised-choosing one in which the fibres run parallel to one another and to the surface of the excised muscle, such as the sartorius or semimembranosus-and connected to a galvanometer of high resistance by means of a pair of unpolarisable electrodes, one being placed at about the centre of the longitudinal surface and the other opposite a transverse section of the muscle made by cutting across the muscle near one end, Du Bois-Reymond showed that a current was produced by the muscle, which passed, in an outside circuit, from the longitudinal surface to the transverse section, and in the muscle, from transverse section to longitudinal surface. It was also shown that if the muscle were tetanised the amount of this current underwent a change, and in the direction of diminution. Du Bois-Reymond termed the current obtained from the resting muscle the natural current or current of rest, and the alteration in it produced by stimulation the negative variation. Of other workers who have added to our knowledge, Hermann stands foremost, and is the author of the opposing view, which has steadily gained ground until it is now almost generally accepted, that normal muscle is iso-electric at all parts, and that it will only yield a current, either when injured at some part, or on contraction. If one electrode be connected to an injured part and the other to an uninjured, the galvanometer will show a current passing from the uninjured to the injured part. Or, again, if two iso-electric points be connected to the galvanometer, and a muscle wave then started along the muscle, the galvanometer will show a current passing from the resting part to that in contraction. Further, a less injured or less active part is 
electro-positive to one more injured or more active respectively. Perfectly uninjured and resting muscle cannot be experimented upon because simple exposure of the muscle causes sufficient injury to set up differences in electro-motivity. Still that a perfectly normal resting muscle is iso-electric is practically certain. The greater the care taken in the exposure of the muscle the less are the currents obtainable, and the heart, which can be exposed without injury, shows no current when at rest. Conversely if a muscle be purposely injured it yields a current which is in proportion to the injury; and an injured resting heart also gives a current.

\section{EXPERIMENTS TO SHOW THE EXISTENCE OF THE VARIOUS CURRENTS BY PHYSIOLOGICAL MEANS}

Experiment 1.-Galvani's experiment of contraction with metals. Solder a piece of copper wire to the end of a piece of zinc wire. The wires should be fairly"thick and about $6 \mathrm{~cm}$. in length. They may then be bent round into the form of a $U$. Pith a frog, remove the skin from the back of the thigh and dissect out the sciatic. Lift up the nerve on the end of one of the wires, and with the end of the other tonch any part of the frog. At each contact the museles of the leg give a twitch. In the more classical form of the experiment the vertebral column is cut across just above the sacrum, the whole of the abdominal viscera removed and the skin stripped off the legs. The urostyle and the muscles attaching it to the ilia are removed so as to expose both sciatic plexuses. An s-shaped hook of copper wire is then passel round both plexuses, and by this the frog is suspended to a clean iron tripod. If the tripod be now tilted so that any part of the legs of the frog touches the metal a twitch occurs in both legs.

This was Galvani's first experiment, which he brought forward to prove the existence of animal electricity, and which led to the celebrated controversy between him and Volta. Volta proved-by the invention of the voltaic pile-that the contraction was in reality due to the current caused by contract between two dissimilar metals.

Experiment 2.-Galvani's experiment of contraction without metals. Very carefully dissect out a sciatic nerve and gastrocnemius and place it upon a clean dry glass plate. Lift up the nerve on a glass rod drawn out into the form of a hook and lower the cut end on to the lower end of the musele. With each contact the musele contracts in response to the stimulus started in the nerve by the closure of the current of injury of the muscle. If the experiment does not at onee succeed, injure the lower end of the muscle by touching it with a hot glass rod or wire and then repeat the experiment.

This is the crucial experiment which definitely proved that part of Galvani's views as to the existence of animal currents.

Experiment 3.-Kühne's experiment of contraction without metals. Place a clean glass plate so that it projects about one inch over the edge of the table. Make two rolls of china elay moisiened with normal saline and place then parallel to each other, so that they project beyond the edge of the glass plate, and turn down the projecting euds till they hang below the 
plate (fig. 116). Make a nerve muscle preparation-the sciatic nerve and the whole of the leg below the knee-and place the cut end of the nerve on one clay pad and the middle of the nerve on the other. Great care must be taken of the nerve during its preparation. Place a little normal saline in a watch glass and lift this up until both clay pads touch the fiuid. At the contact the leg muscles give a twitch, due to the closure of the current of injury of the nerve.

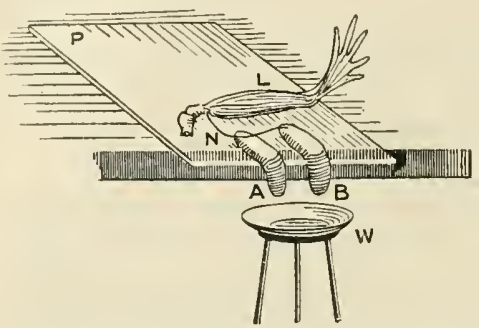

Fig. 116.-Kühne's Expeninent of Contraction mithout Mietals.

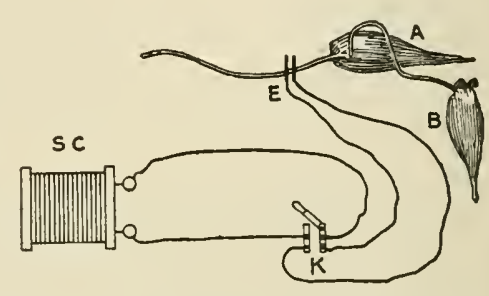

Fig. 117.-Arrajgement of Apparatus for showing Secondary Contraction.

Experiment 4.-Secondary contraction. Dissect out two nerve-muscle preparations and place them on a dry glass plate. Lift up the nerve of one, $\mathrm{B}$, fig. 117, and place it in a loop over the muscle of the other preparation, A. Lay the nerve of A over a pair of electrodes, $\mathrm{E}$, in connection with a Du Bois key, $\mathbf{k}$, in a secondary circuit. Close $\mathrm{k}$ and connect a battery to the primary coil for tetanising currents. Open the key $\mathrm{K}$ and the preparation $\mathrm{A}$ passes into tetanus. It is also found that $\mathrm{B}$ is thrown into tetanus. On closing $\mathrm{K}$ the tetanus of $\mathrm{B}$ ceases.

This experiment is of considerable importance on several accounts. In the first place we can show by physiological means the existence of a current of injury in the muscle of $\mathrm{A}$, for if the nerve of $\mathrm{B}$ be sensitive and it is laid across the muscle of $\mathrm{A}$ and then its cut end dropped on to some other point of $\mathrm{A}$, at the contact the muscle of $\mathrm{B}$ contracts stimulated by the closure of the current of injury of A. In the second place it directly demonstrates the 'negative variation' or current of action of $\mathrm{A}$, for it is due to the production of this current that the muscle $\mathrm{B}$ is stimulated when $\mathrm{A}$ is indirectly tetanised. In the third place it teaches us one very important fact with regard to the nature of the physical events occurring in A while it is being tetanised. This results from observing that $\mathrm{B}$ passes into tetanus when $\mathrm{A}$ is tetanised; and as a muscle does not contract during the time that a constant current is passing through it, but only on make and break, it follows that the current of action of a must be intermittent. Hence although the change of length of A may remain constant, one at least of the physical factors accompanying that contraction is intermittent. By other methods it has been shown that the rate of oscillation of the current of action of $\mathrm{A}$ is the same as that of the excitation. 
By a somewhat similar experiment one muscle may be dircctly stimulated by the current of action of another. Thus if two sartorius preparations be made from a curarised frog and the one muscle pressed tightly against the other, so that at each end one of the muscles projects a little, tetanisation of one muscle at its uncovered end leads to a tetanus of the second.

Experiment 5.-Show the current of action of the heart by excising the whole heart, and having nearly emptied it of blood by tonching it with dry blotting-paper, injure the apex of the ventricle and then place it on a thoroughly dried glass plate. Dissect out a sciatic very earefully and with the leg still attached place it on the heart, so that it crosses the base of the ventricle and its cut end lies on the injured spot at the apex of the heart. With each contraction of the heart the muscles of the leg give a single twitch, being stimulated by the current of action of the heart.

Apply a Stannius ligature to the heart, which is thus brought to a stand. still. With each mechanical stimulation the ventricle gives a beat and its current of action leads to a twitch of the leg muscles.

\section{EXAMINATION OF THE DIFEERENT CURRENTS BY MEANS OF THE REFLECTING GALVANOMETER}

The galvanometer employed for this purpose consists of a pair of suspended magnets which are made very nearly astatic, each being surrounded by a coil consisting of very many turns of fine insulated wire.

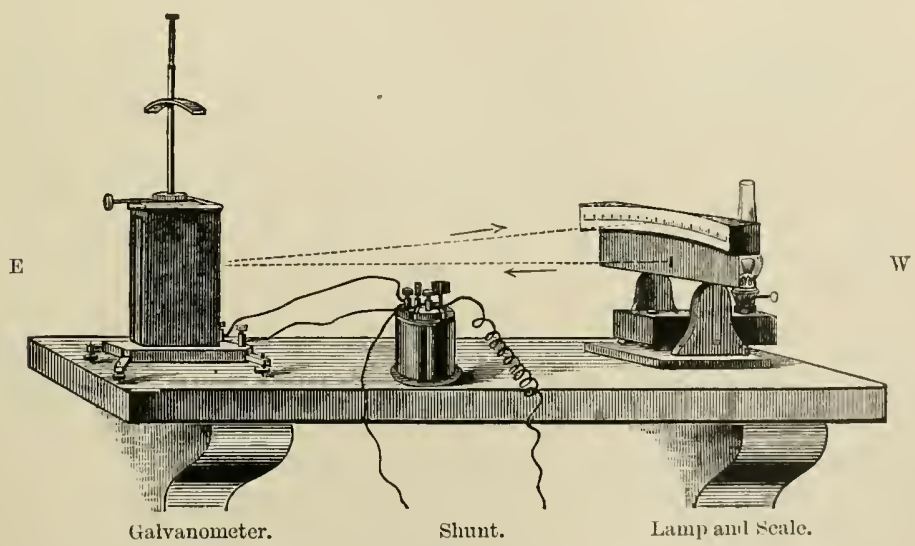

Fig. 118.-Side View of Galvanometer and Shunt, Lajip and Scale

The galvanometer and seale are placed east anl west, and appear as if viewal by an observer standing on the north side and looking soutl ; the path of light is indieated by dotterl lines. The essential parts coneealed by the galvanometer ease are diagrammatieally given in fig. 119. (Waller.)

The two coils are connected up so that the current is sent clockwise through one coil, and anti-clockwise through the other (see 
fig. 119). The resistance of such a galvanometer is very high, from 10,000 to 20,000 ohms, and therefore tends to weaken the current. This does not matter, however, when we are studying the current yielded by a piece of tissue whose resistance is very high, and are employing unpolarisable electrodes whose resistance is also very great. Attached to the upper magnet is a light mirror by means of which a beam of light is reflected, and thus any rotation of the magnet and attached mirror is detected by the movement of the reflected beam.

The galvanometer is set up so that the mirror faces to the west, and the magnets and coils therefore lie in the magnetic meridian.

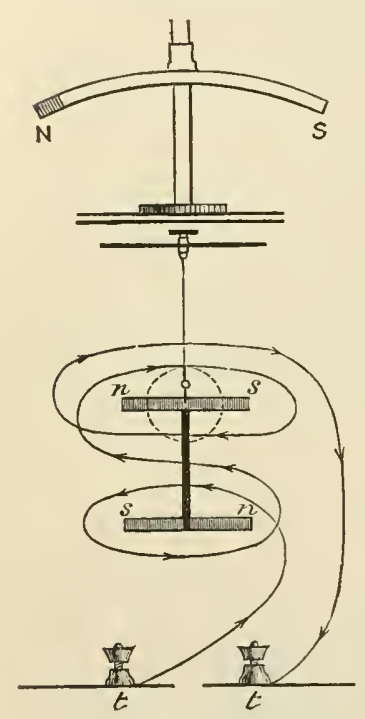

Fig. 119.

Astatic couple of magnets $n s$, $s n$, suspended by a silk fibre and carrying a mirror (indicated by the dotted circle) : the surrounding line and arrows indicate the disposition of the coils: $\mathrm{N} \mathrm{S}$ is the neutralising or coutrolling magnet. All these parts are represented as if vicwed by an observer standing west, i.e. in the position of the lamp in fig. 118. (Waller.)
The coil is provided with a shunt by means of which we can vary the amount of the current allowed to pass through the galvanometer when we are dealing with relatively large currents. This consists of three resistances of $\frac{1}{9}$ th, $\frac{1}{99}$ th, and $9 \frac{1}{9}$, th of the resistance of the galvanometer, and is connected up in parallel with the galvanometer, i.e. as a deriving circuit. By means of a plug we can utilise either of the three resistances, or by leaving out the plug send the whole current through the galvanometer. If the plug be inserted so that the resistance of $\frac{1}{9}$ th is in parallel with the galvanometer then the current is divided, 1 part passing through the galvanometer and 9 parts through the shunt, i.e. $\frac{1}{10}$ th of the total current is sent through the galvanometer. Similarly by means of the other resistances we can send $\frac{1}{100}$ th or $\frac{1}{1000}$ th of the current through the galvanometer. The shunt is further provided with a short-circuiting key, so that all the current can be sent through the shunt and none allowed to pass through the galvanometer.

To observe the movements of the needle a source of light is condensed on to a narrow vertical slit, and the light from this is collected by a projecting lens and then thrown on to the mirror of the galvanometer, and so from this on to the scale (see fig. 118), where it is focussed sharply by adjusting the position of the projecting lens.

First determine the nature of the deflection by sending a small 
current of known direction through the galvanometer. For this purpose arrange the shunt to send $\frac{1}{1000}$ th of the current through the galvanometer and take a small battery made of a zinc and copper wire with wires soldered to them, passing through a cork, and dipping into some weak sulphuric acid in a small tube. The short-circuiting key of the shunt is closed, and then the leads are connected to the galvanometer. On opening the short circuit the spot of light is immediately deflected to one side, because the two wires form a little battery of which the copper is the positive pole and the zinc the negative. Note which galvanometer terminal is connected to the copper wire; you will then know that if in the experiment a deflection occur in the same direction the current causing it enters at that same terminal. Let us suppose that when the spot of light is deflected to the north, the north terminal of the galvanometer is connected with the copper wire, and is therefore positive.

A pair of unpolarisable electrodes are now prepared and tested to show that they are iso-electric by connecting them to the galvanometer and bringing the two clay guards in contact, when no deflection must occur. If there be a slight deflection the electrodes may often be rendered iso-electric by connecting their two terminals with a stout copper wire and then placing the two clay guards in contact, leaving them so for some hours. If much deflection be caused it is better to remake the electrodes.

Now make a semimembranosus and gracilis preparation, taking great care to injure it as little as possible. Place this across the unpolarisable electrodes, so that the centre rests on one electrode and the lower end upon the other. Open the key of the galvanometer and observe if any deflection be produced. It will usually be but slight, and in such direction to show that the tibial end is electro-negative to the equator. Now remove the muscle and injure the tibial end by touching it with a hot wire. Replace the muscle on the electrodes and again open the short-circuiting key of the galvanometer. A deflection is now obtained in such direction as to show that the injured end is negative to the non-injured surface. This is the current of injury. Next apply a pair of electrodes to the upper end of the muscle and tetanise it. The galvanometer swings in the reverse direction and may nearly reach the zero of the scale. This is the current of action, and its direction shows that the injured end has become less electro-negative relatively to the centre of the muscle.

It is further to be observed that the diminution of current persists throughout the whole time the muscle is tetanically contracted, though, as previously found (p. 148), the current of action is of an alternating 
character. This is because the galvanometer cannot respond to the rapid alternations, the inertia of the magnets being too great, and it therefore only gives a constant deflection proportional to the mean value of the alternations.

The production of this current proves that a part of a muscle which is contracting is electro-negative to one which is at rest or in a less degree of contraction. The injured part contracts less forcibly than the non-injured, and it is because the injured part does not respond so well to stimuli that its negativity remains practically constant, whilst the negativity of the central active part becomes greater relatively to its previous state.

\section{MEASUREMENTS OF POTENTIAL}

The method adopted by Du Bois-Reymond for measuring the E.M.F. of an animal current was that of compensation, in which the current through the galvanometer is balanced by a fractional part of a constant current of known E.M.F. sent through the galvanometer in the reverse direction. The principle of the method is illustrated by fig. 120. A Daniell battery is connected to the two ends of a long platinum wire, A C, a commutator, $\mathrm{K}$, being interposed so that the direc-

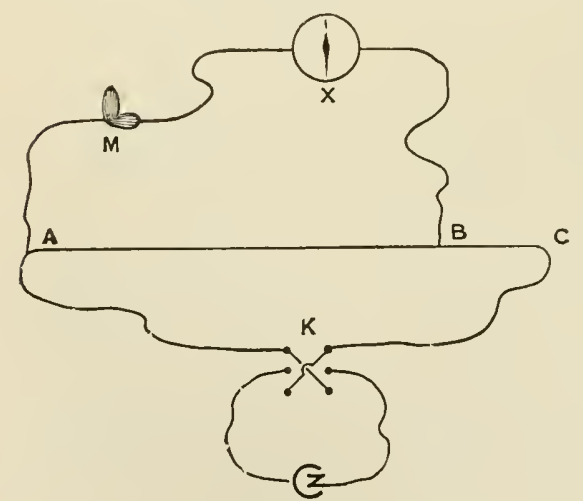

Fig. 120.-Plax of Du Bois Reynonid's Method of measuring the Muscle Currents.

tion of the current through the wire can be reversed. The current to be measured is sent through the galvanometer and a part of the platinum wire, viz. A B, the connection at $\mathrm{B}$ being movable. The muscle current in the arrangement of the figure passes from $\mathrm{A}$ to $\mathrm{B}$, and the Daniell current is, by placing the commutator in the proper position, directed from B to A. A position of $B$ is now sought at which the galvanometer remains undeflected, when it follows that the branch circuit from the Daniell through the galvanometer is equal to the muscle current, and consequently that the E.M.F. between $\mathrm{B}$ and $\mathrm{A}$ due to the Daniell is equal to the E.M.F. of the muscle current.

With the high external resistance the difference of potential between $\mathrm{A}$ and $\mathrm{C}$ is practically that of the Daniell cell ; and as the wire 
$\mathrm{A} \mathrm{C}$ is uniform the fall of potential is regular, and therefore the E.M.F. between $\mathrm{A}$ and $\mathrm{B}$ is $\frac{\mathrm{AB}}{\mathrm{AC}}$. $\mathrm{E}$, where $\mathrm{E}$ is the E.M.F. of the Daniell. In any particular experiment the position of $\mathrm{B}$ will be found to lie quite close to $\mathrm{A}$, so that the ratio of $\frac{\mathrm{AB}}{\mathrm{AC}}$ is somewhere about 03 .

At times instead of using a simple wire, $\mathrm{A}$ C, for this purpose, two variable resistances are employed: one a low resistance representing $\mathrm{A} \mathrm{B}$, the other a higher resistance representing $\mathrm{B} \mathrm{C}$ : but the principle is just the same as for the wire.

For another method of measuring the E.M.F. see p. 155.

\section{EXAMINATION OF THE HEART CURRENTS}

(1) Pith a frog, expose its heart, and apply the Stannius ligature. Roll out the clay pads of the electrodes to sharp points and apply one to the base of the heart, the other to the apex. On opening the key of the shunt no deflection occurs. The resting heart is therefore iso-electric. Remove the apex electrode, and injure the apex by touching it with a hot wire, then replace the electrode. On opening the key there is now a deflection which shows the injured spot negative to the base or any other non-injured part. Stimulate the heart mechanically near the base. With each contraction thus caused, there is a diminution of the deflection of the galvanometer. Therefore the base becomes negative to the apex. This is the current of action of the heart.

(2) A freshly excised heart is taken and most of the blood removed by soaking it up with filter paper. It is then laid across the electrodes, one touching the base, the other the apex. With each spontaneous beat of the heart there is a deflection of the galvanometer. This is found to be in such a direction as to show base becoming negative to apex.

On a beating heart exposed in situ it has been shown by the capillary electrometer that the electromotive changes with each beat are diphasic in character. It is found that first the base becomes negative to the apex and then the apex to the base. If the apex be injured it then becomes monophasic, base negative to apex with each beat. The injured part does not contact normally, and therefore does not become as negative as the non-injured during its contraction.

If the base be injured then its potential remains unaltered and apex becomes negative to base with each beat. It was in order to render the change monophasic that the apex was injured in the preceding experiment (1). 


\section{THE OAPILLARY ELECTROMETER}

The capillary electrometer consists of a piece of glass tubing with its end drawn out to a fine capillary of an internal diameter of about $30 \mu$. This is filled with mercury and then immersed in diluted sulphuric acid (1 part to 6 of water) contained in a second tube, in the bottom of which is some mercury which serves by a platinum wire fused in the glass to make contact with the acid. The mercury in the upper tube is connected by a
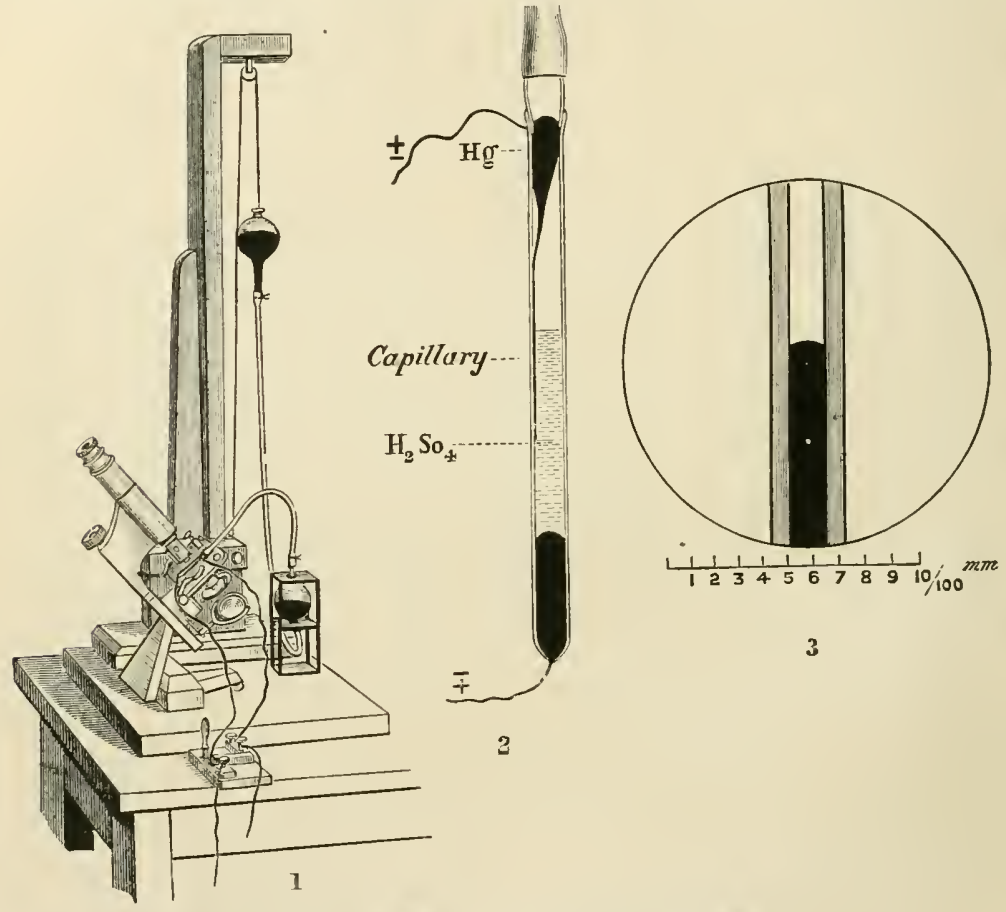

3

Fig. 121.-Lippuann's Capillary Electrojieter.

1. Prcssure apparatus and microscope, on the stand of which the capillary tube is fixed.

2. Capillary tube dipping into $\mathrm{H}_{2} \mathrm{SO}_{4}$ in a surrounding tube, and in conuection with pressure apparatus (the mercury in the lower part of the surrounding tube serves only to establish conuection with the platinum wire).

3. The capillary tube and column of mercury as seen in the field of the microscope. (Scale in $\frac{i}{200}$ th $\mathrm{mm}$.) (Waller.)

second platinum wire which forms the other electrode. The changes of position of the mercury surface in the capillary are watched under a micro. scope. The upper tube is connected by stout rubber tubing to the lower of two reservoirs containing mercury, by altering the relative heights of which pressure can be exerted upon the surface of the mercury. With a constant pressure the mercury in the capillary is brought to a certain position in which the capillarity is exactly balanced by the pressure. If a current be 
now sent through the capillary it is found that the mercury moves in the direction of the current. This movement is due to the altered surface tension brought about by the current. Thus supposing the current passes along the capillary from mercury to acid, the surface tension falls and the mercury moves down the tube. If the current travel from acid to mercury the surface tension rises, and the mercury moves up the tube until a new position is found in which the external pressure is again balanced by the capillarity. By increasing the pressure on the surface of the mercury the mercury may be again driven down the tube till the original position is once more reached. The difference in level of the mercury in the pressure apparatus is a measure of the capillarity, and therefore of the difference of potential. By measuring the pressure when the position of the mercury in the capillary is brought back to its initial position while a current is still passing through it, the instrument may be graduated and can then be directly used as a measurer of small currents. Its great advantage lies in that its electrical capacity is very small, and it can thus show very rapid changes of potential. There is no latency with the instrument and no after-oscillation.

It is to be noted that this instrument measures electrical pressure, i.e. potential, whereas the galvanometer is a current-measurer.

Experiment 6.-Employ the capillary electrometer for the following experiment. Pith a frog, excise its heart and place it on a pair of unpolarisable electrodes with the apex on one electrode and the base on the other.

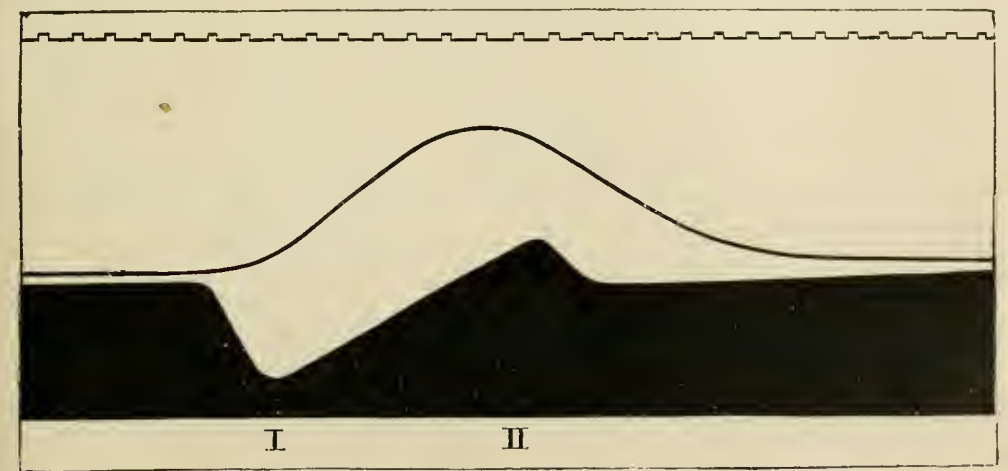

Fig, 122.--Frog's Heart. Diphasic Variation.

Simultaneous photogram of a single beat (black line) and of the accompanying electrical change, indicated by the level of the black area, which shows the varying level of mercury in a eapillary clcctrometer. The base of the ventricle is connected with the mercury. I, First plase, baso negative to apex. II, Sceond phase, apex negative to base. (Waller.)

To prevent the contact between electrodes and heart shifting during the beats the connection between each electrode and heart should be made by a thread moistened in normal saline. The electrodes are connected to the electrometer through a Du Bois key, so that the electrometer can be short-circuited at any time.

If the mercury be watched it will be found to move with each beat of the heart. 
Instead of watching the changes with a heart beating spontaneously, the effect of a single beat may be observed by first applying the Stannius ligature and then mechanically stimulating the ventricle. With each contraction the mercury will move so as to show that the part stimulated becomes first negative and then positive to the other part.

Examine the arrangement of a second electrometer which is fitted up to record the movement of the mercury meniscus photographically. The capillary is brightly illuminated in a projecting lantern and placed in front of a projecting lens by means of which a vertical image of the mercury is thrown on to a vertical slit, part of which it covers. A photographic plate is moved by clockwork behind the slit, and so records the vertical movements of the mercury meniscus.

The result of such an experiment is given in fig. 122. It shows that the electrical variation occurring in a single contraction of the uninjured heart is diphasic in character. In the first phase ( 1, fig. 122) the base was negative to the apex; in the second phase, II, apex was negative to base.

Experiment 7.-Paradoxical contraction. Pith a frog and expose the sciatic nerve, which at its lower end will be found to split into two branches: one, the tibial nerve, supplies the gastrocnemius, and the other, the pero-

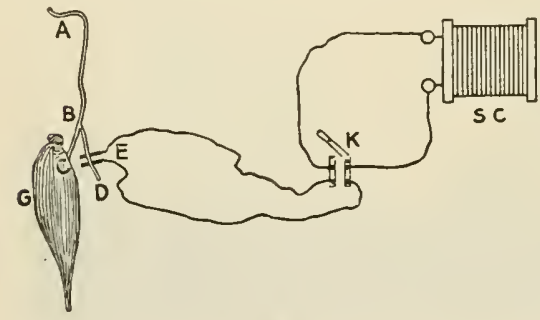

Fig. 123.-Arrangement of Apparates to show the Paradoxical Contraction. neal nerve, supplies the peroneal muscles. Follow the peroneal nerve a little way down the leg and cut it through. In this way is obtained a piece of the sciatic, A B (fig. 123), with the upper ends of its two main branches, one, B c, rumning to the gastrocnemius, $\mathrm{G}$, the other, B D, the isolated piece of the peroneal. Place a pair of electrodes connected with the secondary coil under в 1. With each shock sent through B D the gastrocnemius contracts.

This result is due to a part of the electrotonic current set up in the peroneal nerve passing through the fibres to the gastrocnemius as they lie side by side in the sciatic, $A B$. It is not due to an escape of the current because the result is prevented by tying a ligature round the peroneal immediately above the electrodes sufficiently tightly to injure the fibres without, however, cutting through the nerve, which could therefore still act as an electrical conductor and permit escape of current. Moreover the contraction is not due to the negative variation sent along the peroneal fibres as a result of the stimulation, because it is not produced when the peroneal nerve is stimulated mechanically. 


\section{CHAPTER XVI}

SCHEMA OF THE CIRCULATION. THE SPHYGMOGRAPH

THE flow of the blood through the arteries, capillaries, and veins follows the laws which govern the flow of any fluid through a system of tubes, so that we are able to illustrate many of the features of the blood flow upon an artificial arrangement of tubes drawn up in imitation of the circulation. Such an artificial system is known as a Schema of the circulation. On examining the conditions of flow in the blood vessels, we find that if we expose an artery it feels hard and distended to the touch, and that synchronously with each heart beat it swells and becomes harder. If we cut into an artery the blood spurts out with considerable force which carries it to some distance, and in addition the outflow is of variable rate, for with each beat of the heart the flow is markedly accelerated. To stop the flow it is necessary either to tie or compress the vessel at some point nearer to the heart than the orifice which has been made into it. If, on the other hand, we lay bare a vein, it is found to be collapsed and very readily compressed, and when its cavity is completely obliterated by compression it swells up on the side farthest from the heart. If we cut into a vein the blood flows from it at a good rate, but with slight force and in a constant stream, and to stop this flow it is necessary to tie the vein at any point of the vessel further from the heart than the incision. There are thus many great differences in the characters of the flow from an artery and vein respectively. This change in the nature of the flow is brought about either at the commencement of the capillaries or in the small terminal arteries, for if we examine the capillaries in a living animal under a microscope, the flow is found to be constant and not alternately fast and slow. As arteries subdivide, though each branch may be much smaller than the main trunk, yet the total transverse sectional area is invariably found to be greater than that of the main artery. Again, when we pass from the small arteries to the capillaries, though each capillary is of very small sectional area, their number is so immense that their total sectional area is many hundred times greater than that of the arteries 
from which they have sprung. In examining the flow of a fluid along a tube, it is found that the resistance offered to the flow becomes progressively greater as the diameter of the tube along which it is forced is diminished. The resistance of the system of capillaries and minute arteries must therefore be very great, and this high peripheral resistance explains many of the important facts that we know of the distribution and flow of the blood in the various parts of the body.

Other important points for us to note at the commencement of our study of the physical characters of the circulation are: (a) that the arterial walls are highly elastic, and $(b)$ that the source of the energy required to propel the blood through the vessels is the rhythmic contraction of the heart.

By imitating these three features of the circulation in an artificial schema, we can reproduce and study many of the important

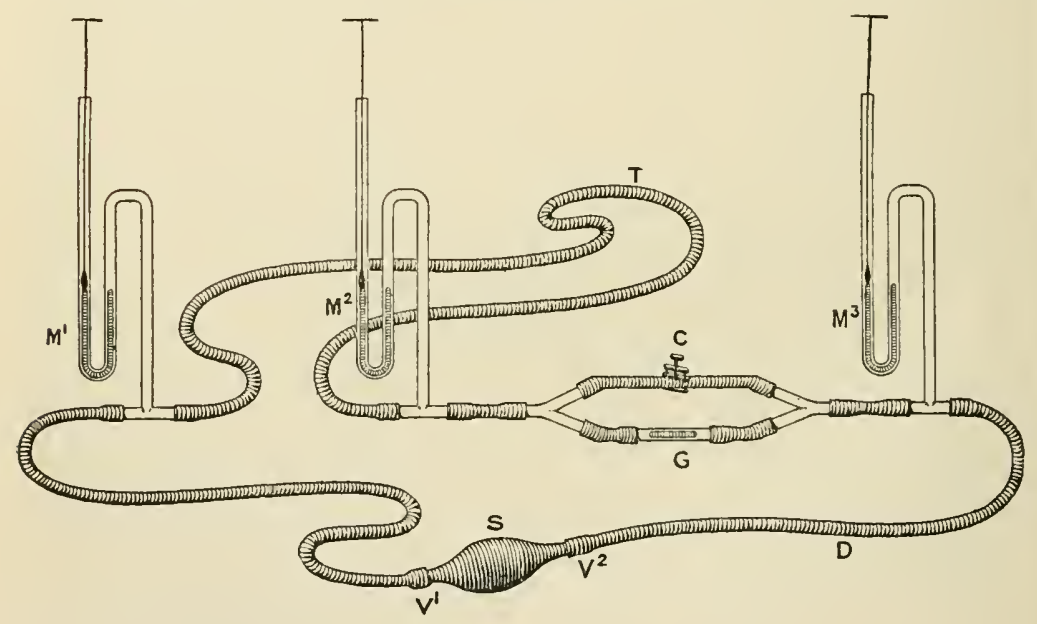

Fig. 124.-Schema of the Circulation.

phenomena presented to us by the circulation. Thus in the schema shown in fig. 124 the heart is represented by the enema syringe $\mathrm{s}$, which is provided with two valves, $\mathrm{v}^{1}$ and $\mathrm{v}^{2}$, which permit the flow of the fluid from $\mathrm{v}^{2}$ to $\mathrm{v}^{1}$, but not in the reverse direction. The arterial system is represented by the rubber-tubing $\mathrm{V}^{1} \mathrm{~T}$, upon the course of which two manometers, $\mathrm{M}^{1}$ and $\mathrm{u}^{2}$, are inserted. The manometer consists essentially of a $\mathbf{U}$-tube held in a vertical position. The bend of the $\mathbf{U}$ is filled with mercury, and one limb which remains open to the air carries a light float which rests on the upper surface of the mercury and accurately follows its movements. The upper 
end of the float is provided with a horizontal arm acting as a writing point, and thus the variations in the level of the surface of mercury can be recorded upon a moving surface. The other limb of the $\mathbf{U}$ is placed in communication with the interior of the tube $\mathrm{T}$ by means of a glass $T$-piece. One of these manometers is placed on the tubing $\mathrm{T}$ near to the syringe $\mathrm{s}$, the other at the end of the tubing, which terminates in two branches, one passing through a short piece of tubing which can be blocked by the screw clamp c; the other passes to a piece of glass tubing, G, whose centre is tightly packed with glass wool. The two branches then reunite and are connected to a third manometer, $\mathrm{x}^{3}$, which latter is in its turn connected to the syringe $\mathrm{s}$ by the rubber-tube $\mathrm{D}$. This last piece of tubing is of wider bore and its walls are thin so that it may represent the veins. In using the apparatus the three manometers are arranged to write their movements vertically over each other on a recording surface. By this apparatus we are able to study the changes in pressure as we vary the conditions of the experiment.

Experiment 1.-First fill the schema with water by disconnecting at $\mathrm{v}^{2}$ and placing both orifices under water. By pumping the syringe, water is then driven through the tubes until all the air is displaced. The tube D is then compressed and a little more water forced in until the level of the mercury in the free limbs of the manometers lies about $2 \mathrm{cms}$. above that of the other limb. The trube $\mathrm{D}$ is then reconnected to the syringe. The experiment may now be carried out in the following way: (a) Leave the clip c open, when we shall be studying the flow along a closed system of elastic tubes of wide bore and consequently offering but little resistance at each point. Now imitate the beating of the heart by rhythmically compressing the syringe $\mathrm{s}$, at first slowly and then gradually increasing the rate of the rhythm.

On studying the tracings obtained the following points can be made out:-

(1) The manometer $\mathbf{M}^{1}$ begins to rise a little earlier than the manometer $\mathrm{M}^{2}$, but later than the instant at which more fluid is forced into the tube from the syringe.

(2) The amplitude of the movement of the second manometer is rather less than that of the first.

(3) The movements of the third manometer $\mathrm{N}^{3}$ are practically the same as those of the other two.

(4) There is no marked permanent excess of pressure at any point of the system over that at any other point.

(5) The effect of the filling (the diastole) of the syringe varies according to the rate at which it is allowed to fill. If the syringe be thick-walled so that it possesses considerable elastic recoil, as soon as it is let free it rapidly dilates and sucks in fluid from the tube $\mathrm{D}$, thus setting up a lower pressure in that tube, which diminution of pressure 
travels as a negative wave backwards along the system, affecting first the manometer $\mathrm{M}^{3}$, and later $\mathrm{M}^{2}$ and $\mathrm{M}^{1}$. The effect upon the manometers is greater the thicker the walls of the tube $\mathrm{D}$, and may be made quite small by increasing the length of that tube and diminishing its thickness. It is also decreased by choosing a syringe which does not dilate rapidly.

In this part of the experiment practically the whole of the fluid travels through the piece of wide tubing $\mathrm{c}$.

(b) In the second half of the experiment, vary the conditions by interposing a high resistance to the flow at one point. This is attained by closing the tube c by the clip, when the whole of the fluid must then pass through the glass tube $G$, which being packed tightly with glass-wool divides up the stream into a great number of minute channels, resembling capillaries, and thus offers a great resistance to the flow. As before, pump fluid through the system, when the following results will be obtained:-

(1) With each systole of the syringe the manometers $\mathrm{NI}^{1}$ and $\mathrm{N}^{2}$ will record a rise of pressure as in the former case, but this sudden rise will be absent from the record of manometer $\mathrm{M}^{3}$.

(2) By the diastole the manometer $\mathrm{x}^{3}$ will alone be affected.

(3) If only one emptying of the syringe be carried out after the oscillations have ceased, the manometers $\mathrm{M}^{1}$ and $\mathrm{M}^{2}$ stand at a higher level than $\mathrm{M}^{3}$, and only slowly fall, while $\mathrm{M}^{3}$ rises until the pressure is once more uniformly distributed.

(4) If, before the pressure becomes thus equalised, the syringe be once more emptied, the manometers $\mathrm{MI}^{1}$ and $\mathrm{M}^{2}$ record a further rise of pressure, i.e. a summation of effect has taken place, which is further increased by a third emptying of the syringe, and so on.

(5) If the emptying of the syringe be continued at a definite rate, at last a condition is reached at which the pressures recorded in the arterial manometers $\mathrm{NI}^{1}$ and $\mathrm{N}^{2}$, instead of continuing to rise, oscillate about a mean pressure. The maintenance of pressure at a mean height means that this pressure is just sufficient to force out through the peripheral resistance during the time of one complete cycle exactly the same volume of fluid as is emptied into the arterial tube at each contraction of the syringe. If the movements of $\mathrm{sx}^{3}$ during this time be next studied, it is found that the only sudden variations are the negative waves caused by the active recoil of the syringe as it returns to its original shape. These rhythmic diminutions in pressure do not travel through the high resistance at $\mathrm{G}$, and therefore do not make themselves felt in the other two manometers. If the emptying of the syringe be repeated sufficiently rapidly, it is found that the mean pressure recorded by the third manometer falls below zero pressure, and takes a negative value. If the movements of the third manometer be not too much masked by these negative 
waves, it is found that the pressure in that part of the system shows a gradual and uniform rise after the filling of the syringe, or, in other words, the outflow through the high peripheral resistance is uniform, though the inflow into the arterial tube $\mathrm{T}$ is intermittent.

It is while working in this latter manner that the schema very closely reproduces the conditions as found in the circulatory system during life. The rhythmic emptying of the syringe into the elastic tube $\mathrm{T}$, which is filled with fluid exerting a high pressure upon its walls, reproduces the emptying of the left ventricle into the overdistended and elastic aorta. The relative distribution of the fluid is the same in both cases, the arterial tube of the schema being overfilled, and its elastic walls stretched, whereas on the venous side the reverse is the case. The most important particular, however, in which the schema reproduces the conditions found in the circulatory mechanism is the conversion of an intermittent flow at the commencement of the arterial tube into a continuous flow through the venous tube. The two factors which lead to this result are:-

(1) The existence of a high peripheral resistance; for when that resistance was absent, i.e. when the tube $\mathrm{c}$ was open, the flow through the venous tube was intermittent; and

(2) The elasticity of the walls of the arterial tube $\mathrm{T}$.

The influence of the latter can be best exemplified by considering what would happen if the tube were replaced by one whose walls were rigid. In such a case, as the fluid is incompressible and the tube, being rigid, cannot be expanded to hold a larger volume, any fluid forced into the commencement of the tube necessitates the ejection of exactly the same volume of fluid from the opposite end at exactly the same instant. Hence, if the inflow to such a tube be intermittent the outflow must also be intermittent.

There is another point of view from which we may advantageously consider the flow of fluid in the schema, namely, by studying the amount of work and the way in which it is applied in setting up the flow, and on the other hand the utilisation of that store of energy. The source of energy is the pumping of the syringe: the loss is caused by the friction the fluid encounters in its flow both against the walls of the tubes and at successive layers within the fluid itself where it is moving at different velocities.

We may, for our present purpose, leave out of account the modifications caused by gravity, for as the fluid is ultimately returned to the same level, they on the whole counterbalance one another. The energy of each emptying of the syringe is at once transferred to the fluid, and is there represented by the velocity imparted to it, and by the pressure set up in it. As soon as the fluid is forced into the 
elastic tube some of the energy is at once imparted to the tube wall and is represented by the stretching of the wall, which, being elastic, can recoil and thus re-impart its store of energy to the fluid from which it received it. This is the most important factor explaining the conversion of the intermittent flow from the syringe into a constant flow through the high resistance $\mathrm{G}$; for though the original source of energy is intermittent in its action, the recoil of the tube wall is constant and lasts as long as the tube is kept over-distended. The elastic wall therefore acts as a means of temporarily storing the energy produced by each forcible emptying of the syringe and subsequently yielding it up again, not as a sudden discharge analogous to the manner in which it received that energy, but as a steady delivery extending over some time.

There is a further phenomenon of the circulation which we can also study upon the schema, namely, the pulse wave. At each emptying of the syringe the fluid ejected at first only produces a distension of the first part of the arterial tube, and as a consequence that part is at a higher tension than that of the piece of tube immediately following. It therefore contracts and forces some of its contents into the next piece of tubing, which in its turn becomes more stretched than the piece next following, and therefore contracts, and so the series of phenomena are repeated along the whole tube. Thus a wave of distension and contraction passes along the tube, which wave is known as a pulse wave. As the pulse wave is in reality a wave of pressure we can study it by recording the changes in pressure at two points on the tube by means of manometers, as in the previous experiment, or we can examine it by recording the changes in the transverse diameter of the tube at two different points, which is, of course, but another way of recording changes of pressure, for the tube is distended in proportion to the pressure of the fluid within it. We will employ this latter method, for it is the one ordinarily employed in recording the pulse wave in man.

Experiment 2.-For this purpose we may modify the apparatus of fig. 124 by omitting the manometers and in their place pass the tube over two grooved metal supports, A and B, fig. 125, firmly fixed to collars held upon a vertical bar. Attached to the two collars are recording levers $L^{1} L^{2}$, which are moved by two light vertical rods with grooved pads, $\mathbf{E}$ and $F$, at their ends where they rest upon the tube. In this way an increase of the diameter of the tube is recorded as a rise of the writing lever. Adjust the two points to write vertically over one another, and record the movements of the two levers caused by a single compression of the syringe, and secondly by a series of regularly repeated compressions.

In such an experiment it is found that there is an appreciable time interval between the instants at which the tube begins to expand 
at the two points, and by a measurement of this time ( $t$ secs.) and of the distance between the two points $(l \mathrm{cms}$.) the velocity of the wave is at once given in cms. per sec. by the quotient $\frac{l}{t}$. To determine the wave length we must divide its velocity by the total time taken for the wave to pass any one point.

Note that in the two tracings the amplitude of the tracing recorded by the lever nearer the syringe is greater than that of the second tracing, which means that the pressure at the second point does not reach so high a value as at the first, some of the energy remaining stored up in the preceding portions of the tube.

In the later portions of the two recorded waves secondary waves are seen, which are due to the reflection of the primary wave from

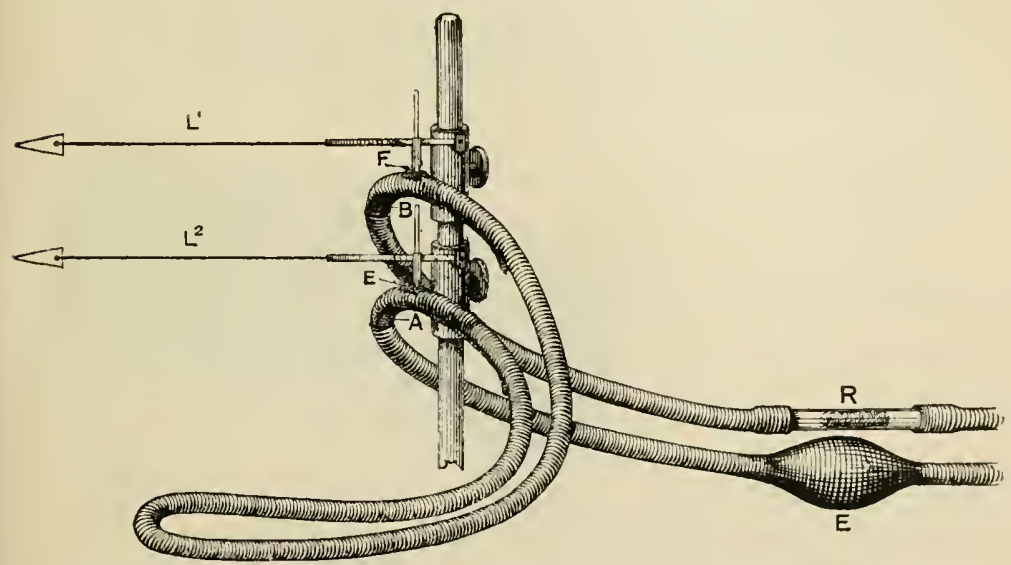

Fig. 125.-Apparatus for Studying the Passage of a Pulse Wave along an Elastic Tube.

the end of the tube, where it impinges on the peripheral resistance. Note that these waves occur at an earlier stage in the second tracing than in the first. In addition to these, other waves are sometimes seen in the early part of the descending portions of the two tracings, which are due to the elastic vibrations of the tube.

Alterations in the characters of the wave, according to the initial pressure of the fluid in the tube, should also be examined. As we wish to study only a single wave, our object can be best gained by compressing the tube beyond the peripheral resistance, so that no fluid can escape. After each compression of the syringe the pressure of the fluid in the tube then remains permanently raised, and we may thus record a series of waves at gradually increasing pressures. It is found that as the pressure is raised the velocity of the pulse wave 
is greatly increased, and so, too, is the wave length. Moreover, as the velocity increases, the secondary waves, due to reflection at the peripheral resistance, occur at an earlier point upon the descending part of the record.

From a consideration of the pulse wave as studied in a schema, we may now pass to an examination of the pulse as occurring in an artery during life. The instruments by which this is effected are termed sphygmographs, and their aim is to record changes in diameter of the artery with each heart beat. These changes in diameter are due to changes of the pressure of the blood in the artery, and consequently in taking a pulse tracing we are in reality recording the variations of blood pressure in that artery.

Experiment 3.-Take a pulse tracing by means of Marey's Sphygmograph, fig. 126.

It consists of a recording lever which writes on a blackened surface moved by clockwork. Fig. 127 shows the principle of the instrument. It is supported

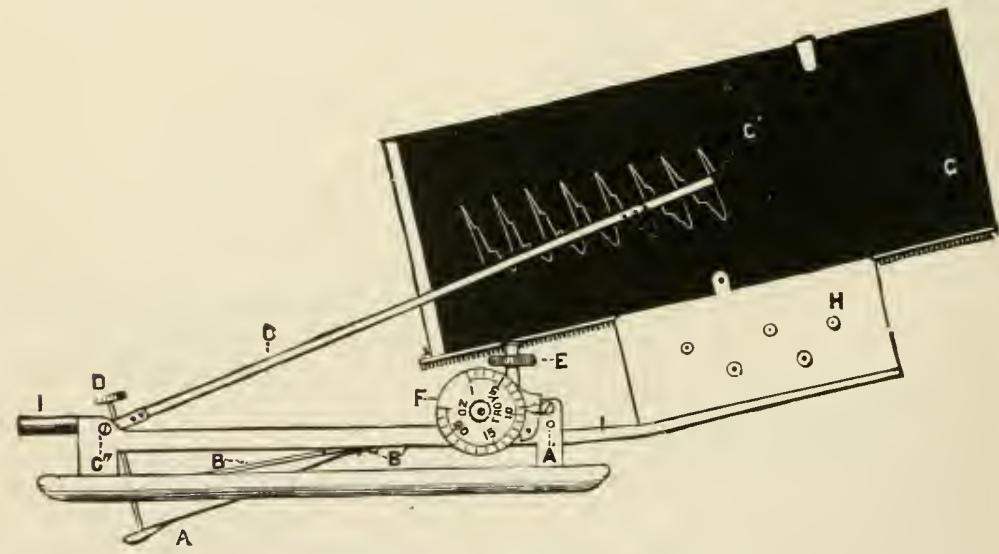

Fig. 126.-Marex's Sphygmograph.

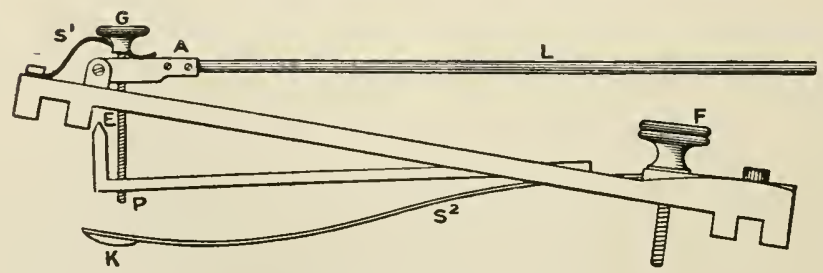

Fig. 127.-Diagram to Show the Arrangement of the Levers in Maref's Sphygmograph.

upon the arm by two runners, between which lies a button, $\mathrm{k}$, placed at the end of a spring, $\mathrm{s}^{2}$. The button is placed over the radial artery at the wrist, 
and its pressure on the artery is modified by the screw $\mathrm{F}$ or by an eccentric as in fig. 126. On the upper surface of the spring rests the end of a screw, $\mathbf{P}$, which carries a small lever terminating in an upright with a knife-edge, $\mathrm{E}$. The knife-edge comes into contact with the recording lever $\mathrm{L}$ at a point very close to its axis and on the metal piece A. This metal piece is not connected to the screw G P. The lever is kept in contact with the knife-edge by a weak spring, $s^{1}$. By turning the screw $G$ the knife-edge is raised or lowered, and so the lever $\mathrm{L}$ is adjusted to record its movements on the recording surface.

In fig. 128 is reproduced a tracing obtained by this method. Each unit is seen to consist of a rapid ascent followed by a more gradual

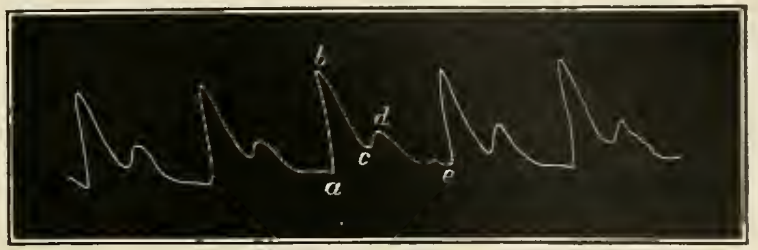

Fig. 128.-Sphygmograir Taken by Marey's Sphygmograph. $a b$, the Primary Wave; $c$, the Dicrotic Notch ; $d$, the Dicrotic Wave ; and $e$, Post-Dicrotic WAVE.

descent. The ascent in a normal tracing is unbroken. In the descent is seen one conspicuous break in the curve at $c$. This is the dicrotic notch. The descent from $b$ to $c$ shows one slight break just above $c$ : this is the pre-dicrotic wave. The descent from $d$ to $e$ shows one or two further waves: these are the post-dicrotic waves. The main wave from $a$ to $b$ is termed the primary or percussion wave.

The record is one of the changes of transverse diameter of the artery, though it is complicated by the fact that the artery is accompanied by veins, and the state of distension of these can exert an effect upon the tracing. By comparison of the pulse tracing of an animal with a simultaneously taken record of the intra-ventricular and aortic pressures the meaning of the different parts has been elucidated. The sudden rise of the lever from $a$ to $b$ is due to the sudden forcing of a fresh quantity of blood by the heart into the elastic aorta. This produces a sudden rise of pressure and distension of the aorta, which is then propagated as a wave of distension over the whole arterial system. The dicrotic notch is immediately preceded by the closure of the semi-lunar valves. The dicrotic wave is therefore a secondary wave produced by reflexion from the surface of the valves. The pre-dicrotic and post-dicrotic waves are waves of oscillation produced in the elastic arterial ivall. They are more conspicuous in pulse tracings taken from "persons in whom the blood pressure is high. 
Experiment 4.-Take another tracing of the movements of the radial artery by means of Dudgeon's Sphygmograph (fig. 129). The form represented in this figure is a modification of the original pattern due to Richardson. A strip of paper blackened on its upper surface is carried under the writing lever by two revolving rollers, the upper one of which is furnished with a series of sharp edges which record lines $2 \mathrm{~mm}$. apart upon the blackened paper. These edges are interrupted so that the horizontal lines

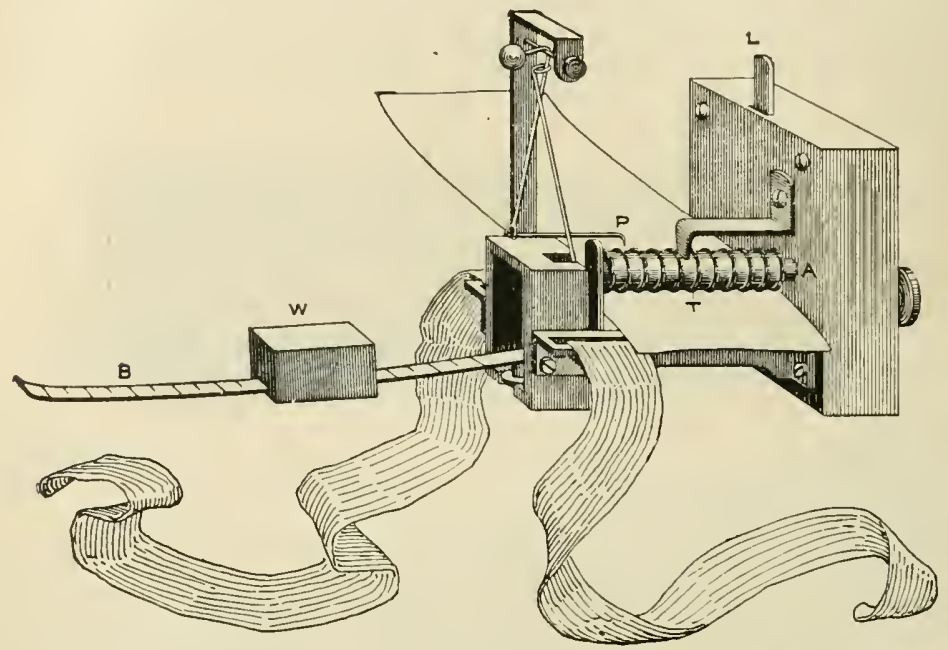

Fig. 129.-Richardoson's Modification of Dudgeon's Sphyomograph.

are broken, and the clockwork is so made that each break follows the preced. ing break after a distance of $2 \mathrm{~mm}$.

The arrangernent of the levers in Dudgeon's sphygmograph is diagram-

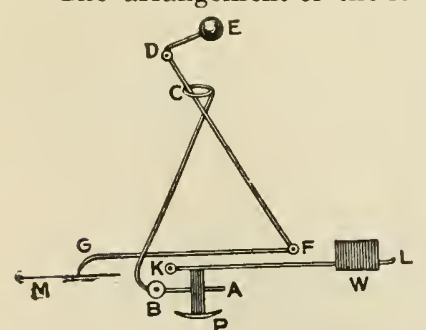

Fig. 130.-Plan of the Leveris in Dudgeon's Sphygmograph. matically represented in fig. $130 . \quad \mathrm{P}$ is a little metal pad which rests upon the artery and which follows the changes of its diameter. It is kept in contact with the skin by aid of a small weight, w, which slides along a rod, $\mathrm{k} \mathrm{L}$, pivoted at $\mathrm{k}$. By varying the position of the weight the pressure exerted upon the artery can be altered at pleasure. $\mathrm{A}$ bent brass lever, c $\mathrm{B}$ A, rotates round an axis at B. Its short arm B A passes through a hole bored in the upright of the pad $\mathrm{P}$. Any movement of $\mathbf{P}$ is therefore communicated to $\mathrm{BA}$, and therefore to $\mathrm{c}$, where it is magnified about five times, because the long arm B $\mathrm{C}$ of the bent lever is about five times that of the short one. A second lever, D C F, rotates round an axis at $\mathrm{D}$ and has attached to it at $\mathrm{F}$ a writing style, $\mathrm{F}$ G. It passes through a ring terminal at $\mathrm{c}$ and is kept in contact with $\mathrm{C}$ by the counterpoise $\mathrm{E}$. The movement at $\mathrm{c}$ is therefore communicated to $\mathrm{DF}$ and magnified at $\mathrm{F}$ about five times because $\mathrm{DF}$ is about five times $\mathrm{DC}$. The movement of the writing point $\mathrm{G}$ is practically that of $\mathrm{F}$. Thus the total magnification of the movement of $P$ is twenty-five times. $M$ is the writing surface. 
Fig. 131 gives two sphygmograms obtained by this instrument. Tracing I is from a rather low-tension pulse, and tracing II from a high-tension pulse. The dicrotic notch is much more conspicuous in tracing I than in tracing II. In II the pre-and post-dicrotic waves are much better marked than in the low-tension pulse. Note further

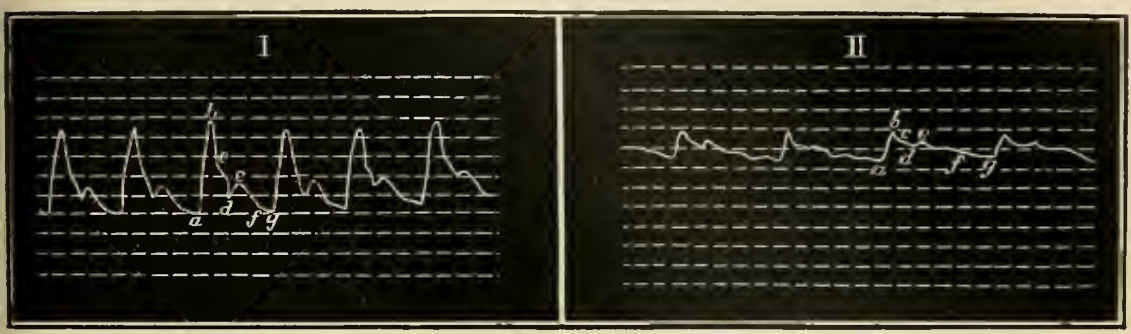

Fig. 131.-Two Sphyguograus Taken by a Dudgeon's Sphygmograph. Tracing I from a Low-tension, il fron a High-tension Pulse.

the difference in excursion between the two and the difference in rate of beat. The clock is so set that it carries the paper through in exactly ten seconds, so that if the total number of waves recorded on the paper be multiplied by 6 the rate of beat per minute is at once given. 


\title{
CHAPTER XVII
}

\author{
DEMONSTRATION OF BLOOD PRESSURE AND ITS NERVOUS
}

REGULATION

For this experiment the following apparatus is necessary (fig. 132). The writing surface consists of an endless roll of blackened paper stretched between two drums, one of which can be rotated at a slow rate by a tangent screw in the ordinary manner. The recording levers consist of a tambour, $\mathbf{T}^{2}$, to record the respiratory movements, a mercury manometer, sI, with float, $w$, and two chronographs, cc. All these writing points are arranged of such a length and in such position that all lie in the same vertical line on the recording surface. Of the two chronographs the lower one is connected to a clock, cl, ticking seconds and a battery, $\mathbf{B}^{2}$. Attached to the escapement of the clock is a wire which each second dips into a cup of mercury (seen vertically below $\mathrm{cl}$ in the figure), thus closing a circuit and actuating the chronograph. The upper chronograph is arranged to record the zero pressure, and is also utilised to mark the instant at which any stimulation is made. For this purpose it is connected in series with a battery $\mathrm{B}^{1}$, the two pillars of a coil $\mathrm{A}$ and a key $\mathrm{K}$. On closing $\mathrm{k}$ the Neef's hammer vibrates, and with each rise and fall of the hammer the chronograph lever rises and falls. To the terminals of the secondary coil a pair of shielded electrodes, $\mathbf{E}$, is connected.

To record the respiratory movements for this experiment a receiving tambour, $\mathrm{T}^{\mathrm{l}}$, is fitted on a horizontal rod with the rubber membrane facing downwards. 'To the centre of the membrane a cork is cemented and the tambour is then held over the rabbit in such a way that the cork rests on the abdomen at a part that moves freely with each respiration. This tambour is connected by rubber tubing, into which a piece is inserted, with the recording tambour $\mathrm{T}^{2}$. With each inspiration the abdomen rises, forces air out of the tambour $\mathrm{T}^{1}$ into the tambour $\mathrm{T}^{2}$, the lever of which therefore rises.

The mercury manometer consists of two vertically placed glass tubes of equal bore, about half-filled with mercury. In one of these a Hoat, $w$, rests on the upper surface of the mercury. The other is connected by a piece of thick-walled pressure tubing to a pressure bottle, $\mathrm{PB}$, which is filled with a half-saturated solution of sodium sulphate. A spring clip, L, controls the connection of the pressure bottle with the manometer. This second tube of the manometer is commected by a lateral piece with a metal tube on which is a tap, s, and this, by rubber tubing of narrow bore and thick walls, can be connected to the cannula in the artery.

The apparatus being prepared, a rabbit is tied down to a holder and put under ether. Its head is then fixed by a Czermak's rabbit-holder. A median incision is made through the skin of the neck for about four inches, so as to expose the larynx at the upper end of the incision. The platysma is ent through in the mid-line. The sterno-mastoid is separated from the sterno- 


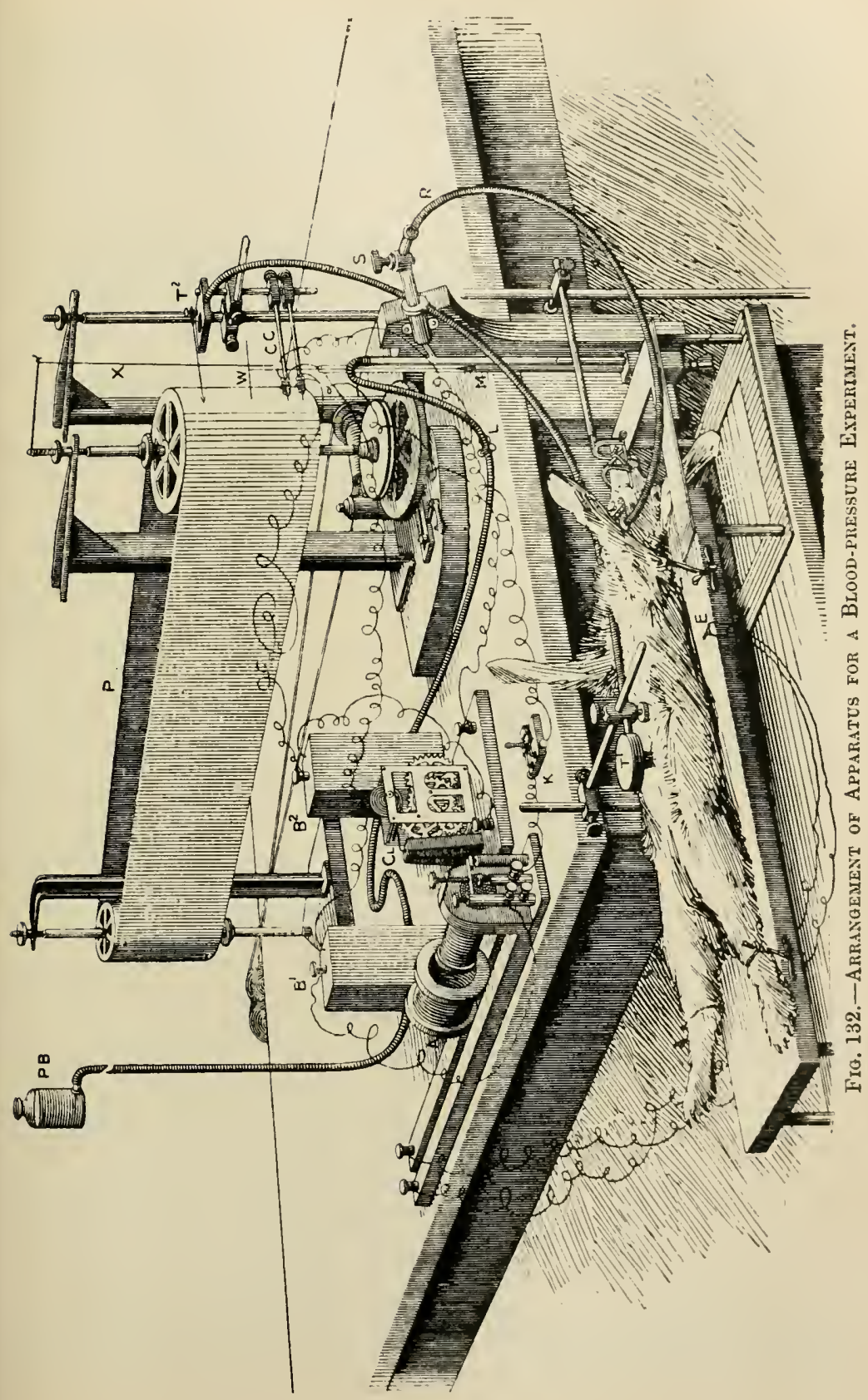


hyoid, which brings into view the carotid artery c, fig. 133, accompanied by a small vein or veins and in relation to several nerves. Superficially lies the descendens noni. Immediately behind the artery lies the vagus, vv, behind and to the inner side the sympathetic, $s y$, and depressor nerve, $\mathrm{D}$. The vagus is first of all separated high up opposite the larynx, and its transverse branch, the superior laryngeal, sL, fig. 126, is isolated. A small nerve, arising either from the superior laryngeal or by two roots, one from this and

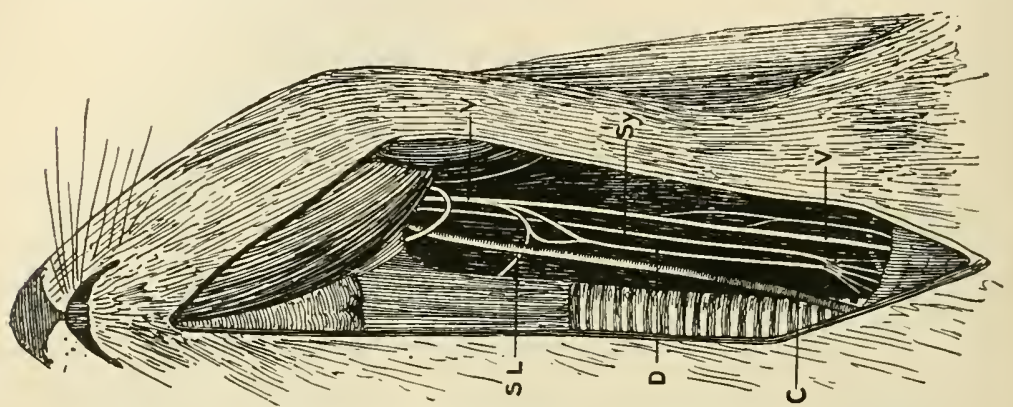

Fig. 133.-Dissection of the Nerves of a Rabbit's Neck. (Cron.)

the other from the vagus, is next sought for. This nerve is the depressor nerve, D. It is isolated for about two inches of its course and a fine silk thread passed under it. The ends of the thread are knotted, so that a loop is formed round the nerve, by which it can be easily lifted from the wound at any time. A double silk thread of another colour is then passed round the vagus and its ends tied together. The vagus of the opposite side is next exposed and ligatures passed round it. The sciatic nerve is then exposed by an incision down the middle of the external surface of the thigh, cutting through the vastus externus, and a ligature is passed round it.

A cannula is now inserted into one of the carotids. The most convenient cannula is of the form shown in fig. 134, B. It consists of a glass bulb with
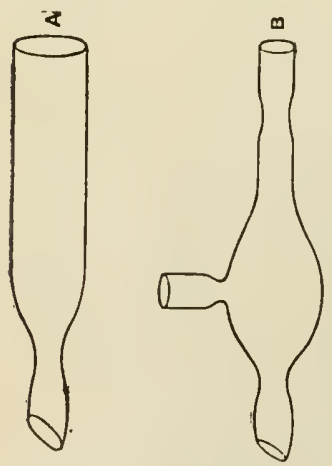

Fig. 134.-Two Foris of CanNULA. three tubes leading from it. One is of fine bore for insertion into the artery. It has a constricted neck and its lower end is cut off obliquely and has rounded margins. The tube opposite this has two constrictions on it, so that rubber tubing does not easily slip off. A short piece of thick-walled pressure tubing is fixed to this and a screw clip fitted over it, so that it can be completely closed. The lateral tube is for attachment to the mercury manometer. To insert the cannula a long piece of the carotid is isolated and a double thread passed under it. One is then tied at the upper end of the isolated piece so as to stop the flow of blood. On the lower end a pair of bull-dog forceps is placed in such a way as to compress the vessel and stop the blood-flow. With a pair of sharppointed scissors the artery is now cut into by an incision which cuts through about one-third of the circumference, and is directed obliquely down. wards. By holding up the flap of artery wall thus made the cannula can easily be inserted into the vessel and tied in by the second thread already placed there, the thread lying in the constriction on the wall of the cannula. 
The animal is placed in a convenient position with respect to the manometer and the cannula connected to the latter. The pressure bottle is raised until it is about five feet above the level of the mercury in the manometer, and with the tap s turned on fluid is run from the pressure bottle through the manometer along the tubing to the cammula, until all the air is displaced from these tubes. The flow from the pressure bottle is then stopped. The writing point of the upper chronograph is brought to the level of that of the float, and consequently draws an abscissa line of zero pressure. It should be noticed that the level of the mercury in the two limbs is not the same, that in the open limb being a little higher than that in the other, because this mercury has to balance the pressure of a column of salt solution equal in height to the difference in level between the mercmy and orifice of the cannula. The clip on the tubing on the free orifice of the cannula is next screwed tight. The clip on the tubing from the pressure bottle is once more opened, but now no fluid can escape. The mercury in the operi limb of the manometer therefore rises, and carries the float with it, nutil the diflerence in level of the mercury in the two limbs balances the pressure of the fluid in the pressure bottle acting on the closed manometer limb. This will be at a level of about 70 to $80 \mathrm{~mm}$. above the zero abscissa line. The increase of pressure inside the manometer is measured by the increase in distance of the two mercury surfaces, but the height recorded by the float is only the movement of the mercury surface in the open limb. If the bore of the tubing forming the two limbs be equal, the rise in the one limb is equal to the fall in the other. Consequently the increase of pressure exerted by the fluid is measured by a mercury column 140 to $160 \mathrm{~mm}$. in height. Therefore in a tracing obtained by the mercury manometer to determine the pressure recorded at any instant, we must ineasure its height above the zero abscissa line and multiply this by 2 , which gives the equivalent height of a mercury column which just balances the pressure.

The bull-dog forceps are now removed from the artery, when the mercury will begin to oscillate up and down, and at about the same level as that it has been raised to by the pressure bottle. The object of the pressure bottle is in the first place to give a convenient means of filling the apparatus with a fluid which when mixed with blood tends to prevent its coagulation. Sodium carbonate or magnesium sulphate solutions are also employed for this purpose; but, on the whole, sodium sulphate is perhaps the best salt to employ. The pressure bottle further performs another very important service in raising the level of the mercury to a height which will approximately represent that of the blood pressure. If this be not done, on connection with the artery the blood itself would have to raise the mercury thus entering the cannula in some volume, not only causing a loss of blood to the animal, but leading sooner or later to coagulation and blocking of the cannula. Placing the pressure bottle too high is also to be avoided, for then some of the fluid will leave the manometer and cannula, and pass into the circulating blood.

In taking the blood pressure by a canmula placed in the carotid we must remember that we are not measuring the blood pressure in the carotid as it exists in life, because we have completely stopped its flow along the vessel. We are really measuring the pressure on the side wall of the artery from which the carotid springs, i.e. either the subclavian or aorta, as the case may be, along which the blood is still flowing in a normal manner.

We may now carry out the experiment in the following manner :

1. Record of the normal tracing.-Two or three short picces of tracing are taken with varying speeds of recording surface. Fig. 135 gives a typical piece of tracing from the rabbit, but the student should 
also examine the tracings obtained from other animals, such as those, for instance, in figs. 139 and 156. In fig. 135 it is seen that there are two distinct series of undulations on the blood-pressure curve-one a small undulation, due to the heart beat, and the second a larger and slower undulation, which is synchronous with respiration. In an animal, such as the rabbit, in which the heart beats very quickly the

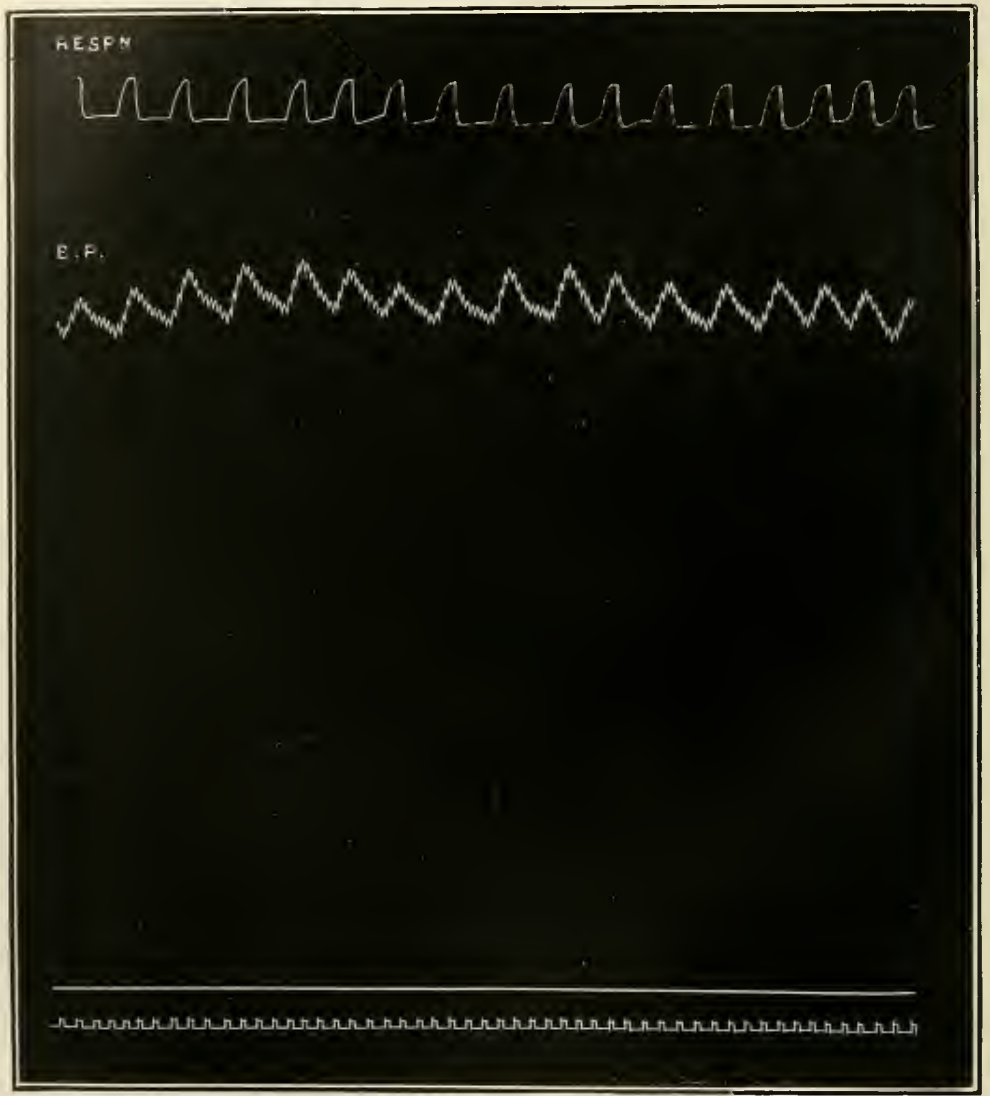

Fig. 135. - Tracing of the Blood Pressure frour a Rabit taken bx the Mercury Manometer. respN, Record of the Respiration; B.P., of the Blood Pressure. The Horizontal Line at the Base shows the Position of the Zero of Pressure. Thme Tracha in Seconds.

oscillations due to the heart beat are small, not, as we shall see, because the variation in total pressure with each beat is small, but because the mercury manometer possesses so much inertia that it is unable to respond to variations which are carried out so rapidly. 
If a record of the heart beat be taken simultaneously with a bloodpressure tracing, it is found that the record of rise of pressure with each beat follows with a considerable latency after the record of the heart itself. The respiratory waves are generally regarded as caused by alterations in the volume of blood admitted to the auricle. During inspiration the greater negative pressure in the thorax draws more blood from the great veins into the thorax, leading to an increased flow through the heart and a consequent rise in blood pressure. This increased flow does not commence at the instant

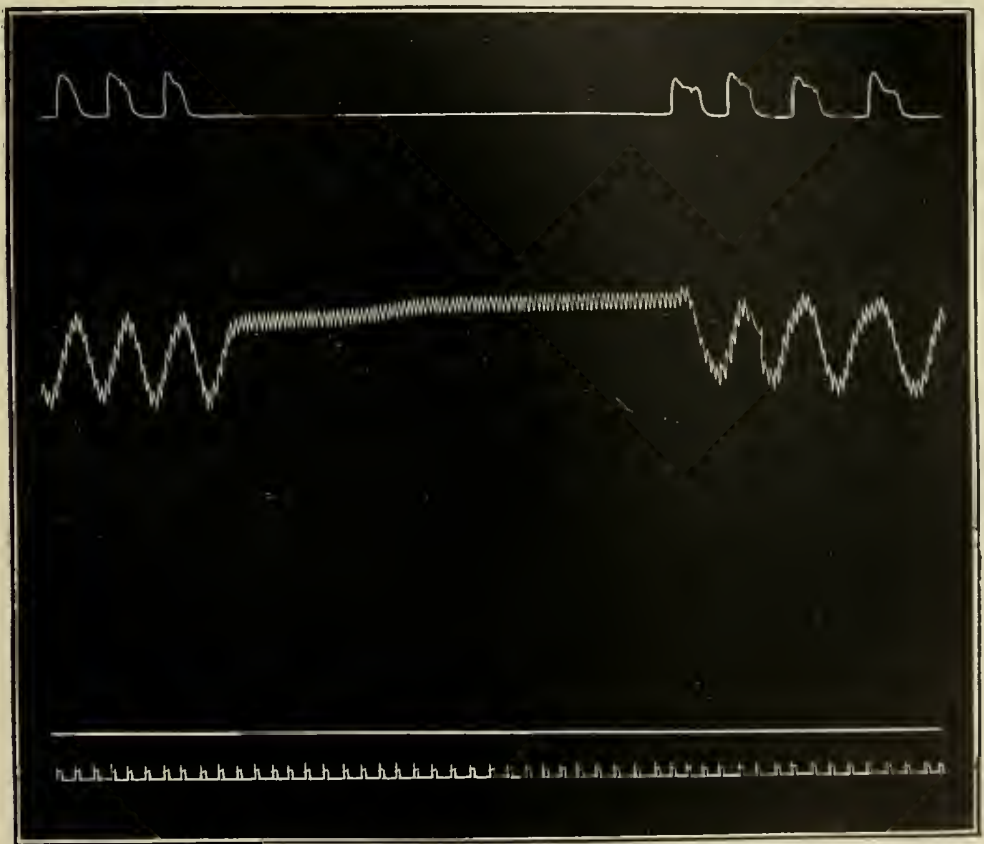

Fig. 136.-Blood Pressure and Respiratory Tracing of a Curariseid Cat under Morphia. Artificial Respiration. Time Tracing Seconds.

inspiration commences, but at a short interval after. The rise in blood pressure, therefore, is not instantaneous with the commencement of inspiration, but follows it after an interval of about two to three heart beats. In expiration there is a diminished blood flow to the ventricle, and therefore a fall in blood pressure, and there is an analogous latency, blood pressure only beginning to fall at an appreciable interval after expiration has commenced. The effect of the respiratory movements in varying the blood pressure is well illustrated by studying the changes during artificial respiration. 
Here we find the exact converse to the results with normal respiration, i.e. a fall in pressure during inspiration and a rise during expiration. In inspiration the thorax is now expanded by pressure from within; and, as the pressure is greater from within than without, the flow into the auricle is impeded. The result is well seen in fig. 136, which is

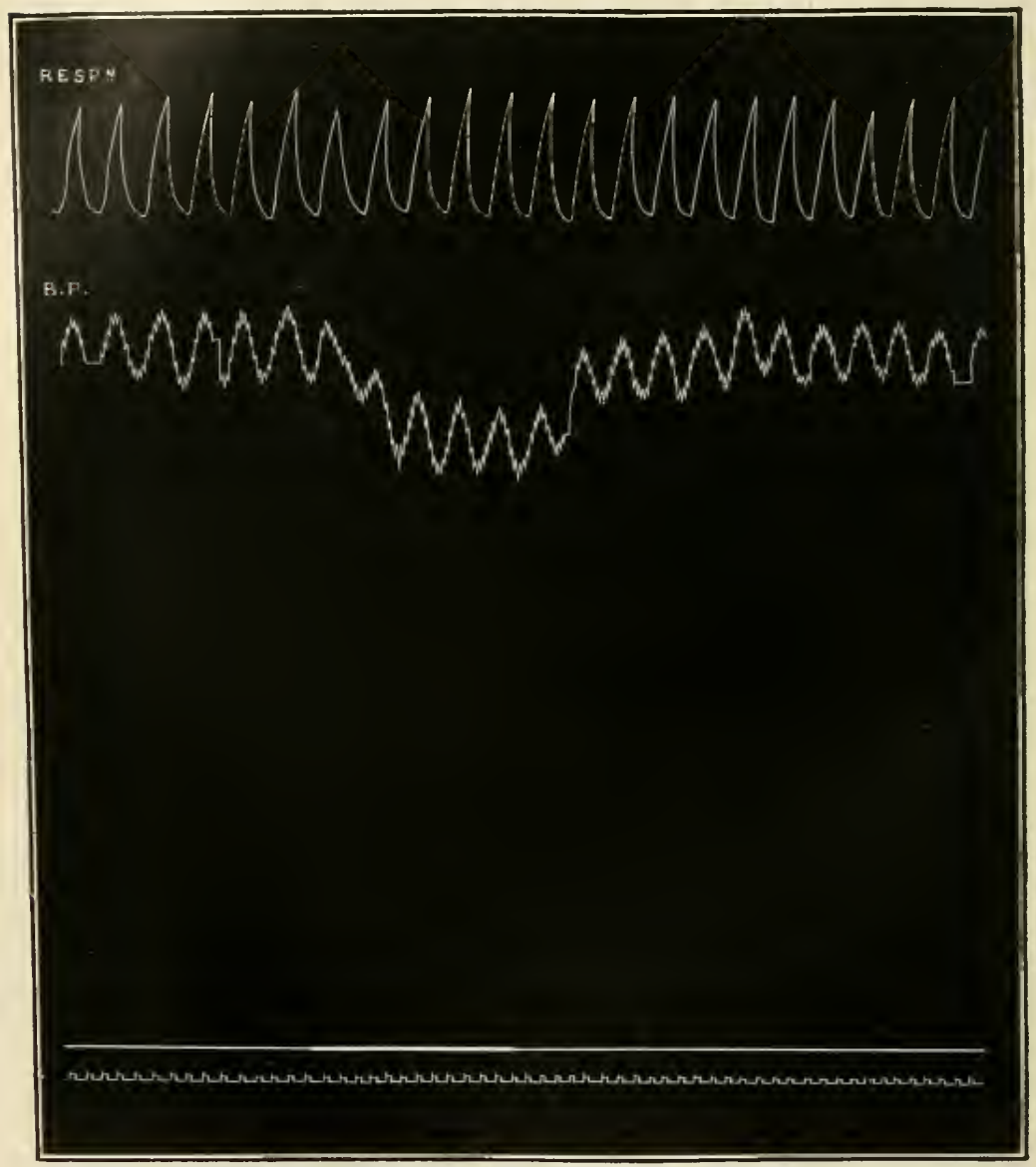

Fig. 137.-Stimulation of the Depressor Nerve in a Rabbit.

Time Tracing Seconds.

from a curarised cat anæsthetised with morphia. Artificial respiration was for a time stopped with the thorax in an expiratory state. The pressure showed a gradual constant rise. Then when a fresh inflation was caused the pressure rose for two heart beats and then rapidly fell to rise again during expiration. The initial rise was due to 
the extra filling of the left auricle by the increased pressure of the air emptying the blood from the lung capillaries. The increased pressure in the thorax retards the flow of venous blood into the right auricle; less, therefore, is sent into the pulmonary artery by the ventricle and the pressure in this artery falls. From this fall in pressure, and because of the obstruction to the flow through the pulmonary capillaries due to the pressure of the air upon them, less blood is delivered into the left auricle, hence less into the left ventricle, and, therefore, a fall in aortic blood pressure.

By measuring the mean height of the tracing in fig. 135, above the zero abscissa line it is seen that the mean blood pressure is $140 \mathrm{~mm}$. of mercury.

2. Stimulation of the depressor nerve.-The nerve is laid on a pair of shielded electrodes, and is stimulated while a record is being taken. The experiment is repeated two or three times, varying the strength of stimulation. Fig. 137 gives a typical result. It is seen that after a short latent period of about two seconds the blood pressure gradually fell from a mean pressure of $140 \mathrm{~mm}$. Hg to one of $124 \mathrm{~mm}$. Hg, but that the character of the tracing was not in any way altered. The heart waves and respiratory waves are still present, and remain as before. When stimulation ceased the pressure once more began to rise after a latent period of about two seconds, and quickly regained its original mean value.

The depressor nerve is an afferent nerve which transmits impulses from the heart, mainly from the endocardium to the vasomotor centre in the medulla. If the nerve be divided and its peripheral end stimulated no result is obtained. Stimulation of its central end produces the result already described. It does not act upon the cardiac centre, but only upon the vaso-motor centre, whose action it so modifies as to cause dilatation of the peripheral blood vessels and therefore a fall in blood pressure. Normally it must act as a kind of safety-valve to the heart under conditions when it becomes difficult for the heart to empty itself against a too high pressure, due either to a higher pressure in the aorta or an overfilling of the ventricle. The increased resistance thus caused stimulates the depressor, the blood pressure falls by dilatation of vessets, chiefly of the splanchnic area, and the heartis now able to empty itself without any special stress.

3. Stimulation of the sciatic nerve.-While recording the blood pressure the central end of the divided sciatic is stimulated, varying the stimulus until a typical effect is produced. The sciatic is chosen simply as a convenient nerve containing afferent fibres, for precisely the same effect is produced with any similar nerve. The result shows a rise of pressure, starting soon after the stimulation commenced, and 
lasting some time after stimulation ceased. This is called a Pressor effect. The only variations in the character of the tracing are due to differences in respiration, which produce secondary effects upon the blood pressure, fig. 138. Often, too, struggling movements are produced, especially if the animal be only lightly under the anæsthetic,

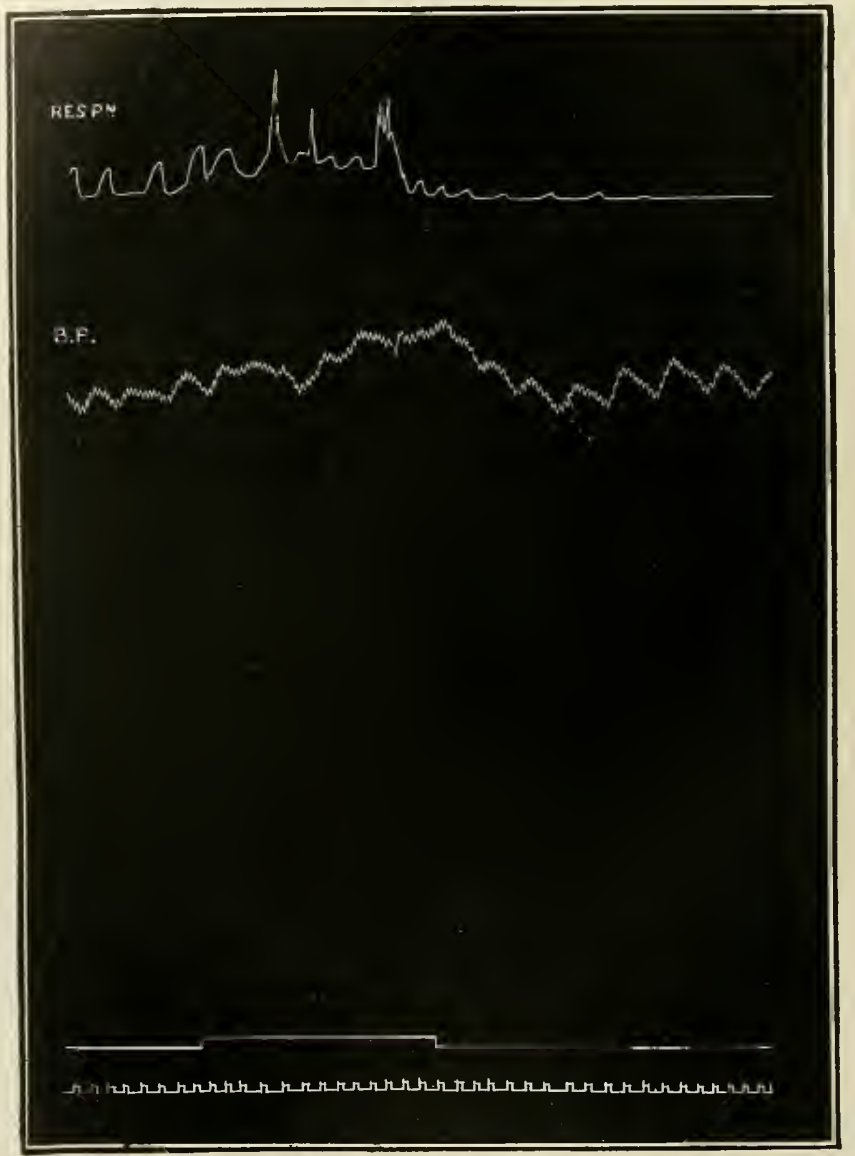

Fig. 138.-Stimulation of the Central End of the divided Sciatic. The Latter Portion of the Respiratory Tracing is imperfectuy recorded.

when with each convulsion the blood pressure rises considerably. The only completely satisfactory method of obtaining a pure pressor effect is to previously curarise the animal. The reflex muscular movements are then absent, and the pressor effect is produced unmasked by the rise of pressure caused by each convulsion. The tracing of fig. 139 
was obtained in this way: it is from a cat under morphia and curarised. It shows that the blood pressure rose from a mean height of $94 \mathrm{~mm}$. Hg to one of $144 \mathrm{~mm}$., but that the characters of the tracing were otherwise retained in a perfectly characteristic manner. This rise in pressure is due to a reflex constriction of the small arterioles, and persists for some time. It is almost completely abolished by section of the splanchnics.

4. Stimulation of the peripheral end of the vagus.-While the record is being taken one vagus is ligatured in two places and cut between the two ligatures. There is, as a rule, no effect upon the blood pressure or respiration. The peripheral end is laid on electrodes

\section{RESP :

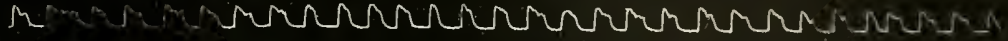 \\ in.

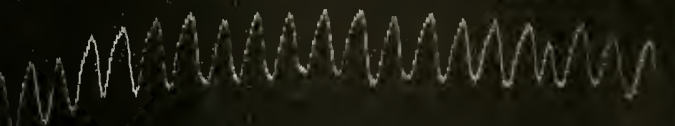

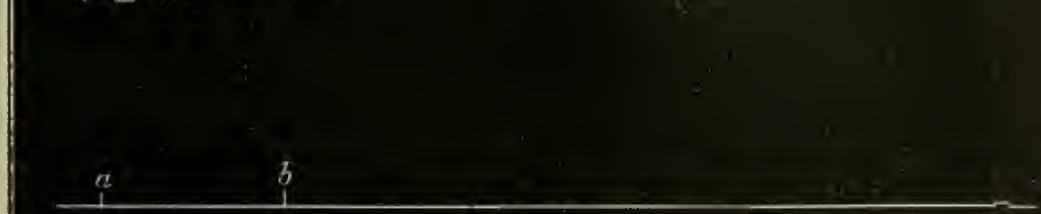

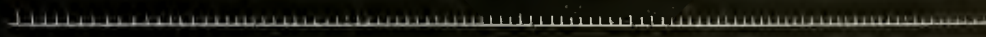

Fig. 139.- Pressor Fffect Pronuced by Stimulatixg the Centrial Eivi of the Sciatic of a Curarised Cat under Morphia. Thme Tracing Seconds. Tracing Reduced to One-half Size.

and stimulated, the stimulation being repeated once or twice with different strengths of stimulus. Figs. 140 and 141 show the effect of such stimulations. Fig. 140 is with weak stimulation, which produced slowing of the heart beat but no stoppage. It is seen that there is a marked fall in blood pressure, which, however, did not commence the instant the stimulus was applied. The variations of pressure now recorded as caused by each heart beat are seen to be rery much larger. This is not due to an increase in force of the ventricular contraction, for, as we have previously seen, the force of the beat is decreased on vagal stimulation (p. 141). Nor, again, is it entirely due to the diminished blood pressure, enabling the heart to produce a 
larger variation with the same force. It is mainly due to the inertia. of the recording instrument. With the rapidly beating heart the manometer could only respond to a limited extent to the change of pressure produced; but now that the beat is slower more time is allowed to the manometer, and its movements therefore:

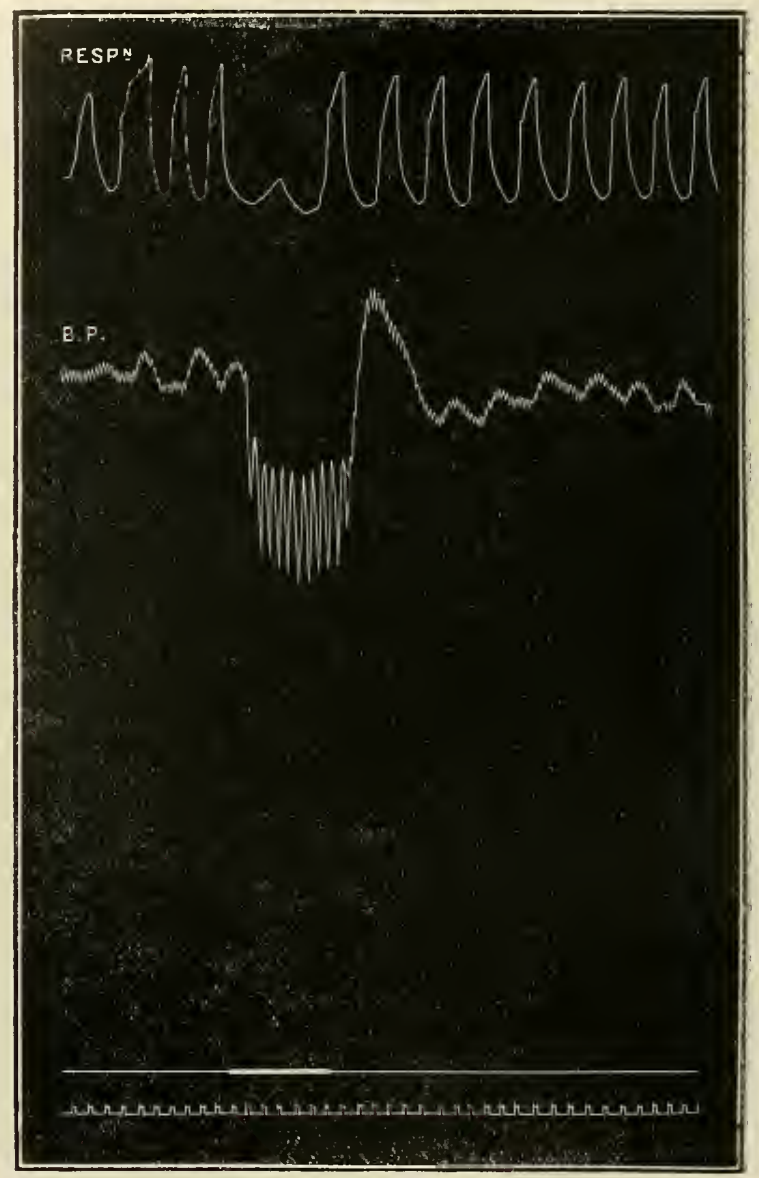

Fig. 140.-Stiavlation of the Peripheral Exi of the Left Vagus in a Rabit.

increase in amplitude. After stimulation ceased the rate of beat very quickly increased, and this, aided by an increase in ventricular force and a partial constriction of the arterioles, led to a rise of blood pressure to a greater height than before; but this was soon followed by a fall, the mean pressure once more attaining its initial value.

Fig. 141 is a similar result, but the stimulation was stronger and 
inhibition more complete. The fall at the first stimulation is seen to be greater, and only a single beat occurred during stimulation. After the stimulus ceased the heart soon regained its rhythm, and the characters of the trace were practically the same as those studied in the previous instance.

The result of a second stimulation $\mathrm{s}$ reproduced because it illustrates another effect which is obtained if the vagus be frequently
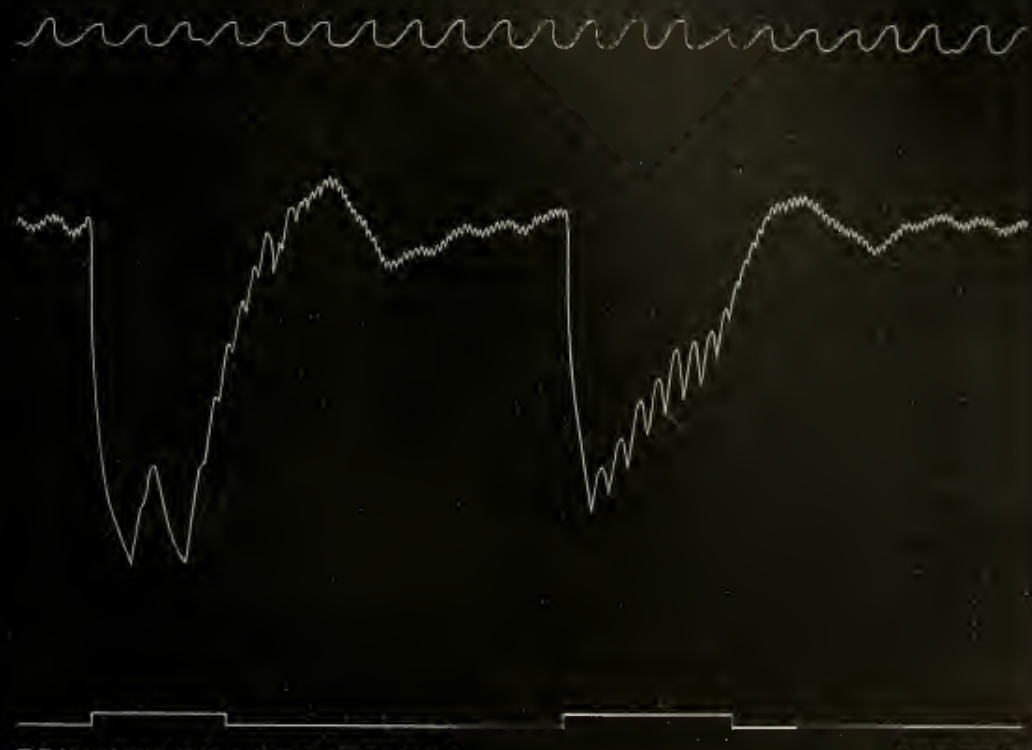

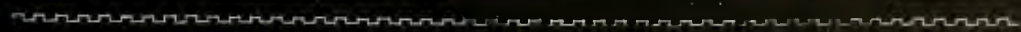

Fig. 141.-Two Successive Stimulations of the Peripheral Eni of tire Right Vagus with the Same Strength of Stinulus. Rarait. Renuced to ONeHaLF Size.

stimulated. It was obtained by stimulating the vagus with the same strength of current as that used for the preceding instance and after an interval of fifteen seconds. It is seen that while the stimulation still continued the heart recommenced to beat and the blood pressure gradually rose. This result is known as the escape of the heart from vagus inhibition. It becomes more and more marked the longer the stimulus is continued, and if stimulation be repeated a few times, at last a stage is reached in which no effect upon the heart nor upon the blood pressure is produced.

5. Stimulation of the central end of the vagus.-The result of such 
stimulation varies with the strength of stimulus employed. As it is an afferent nerve, stimulation of its central end tends to produce a pressor effect. This is the result if the stimulus be weak, and it is accompanied by an increased rate of breathing, which also pro-

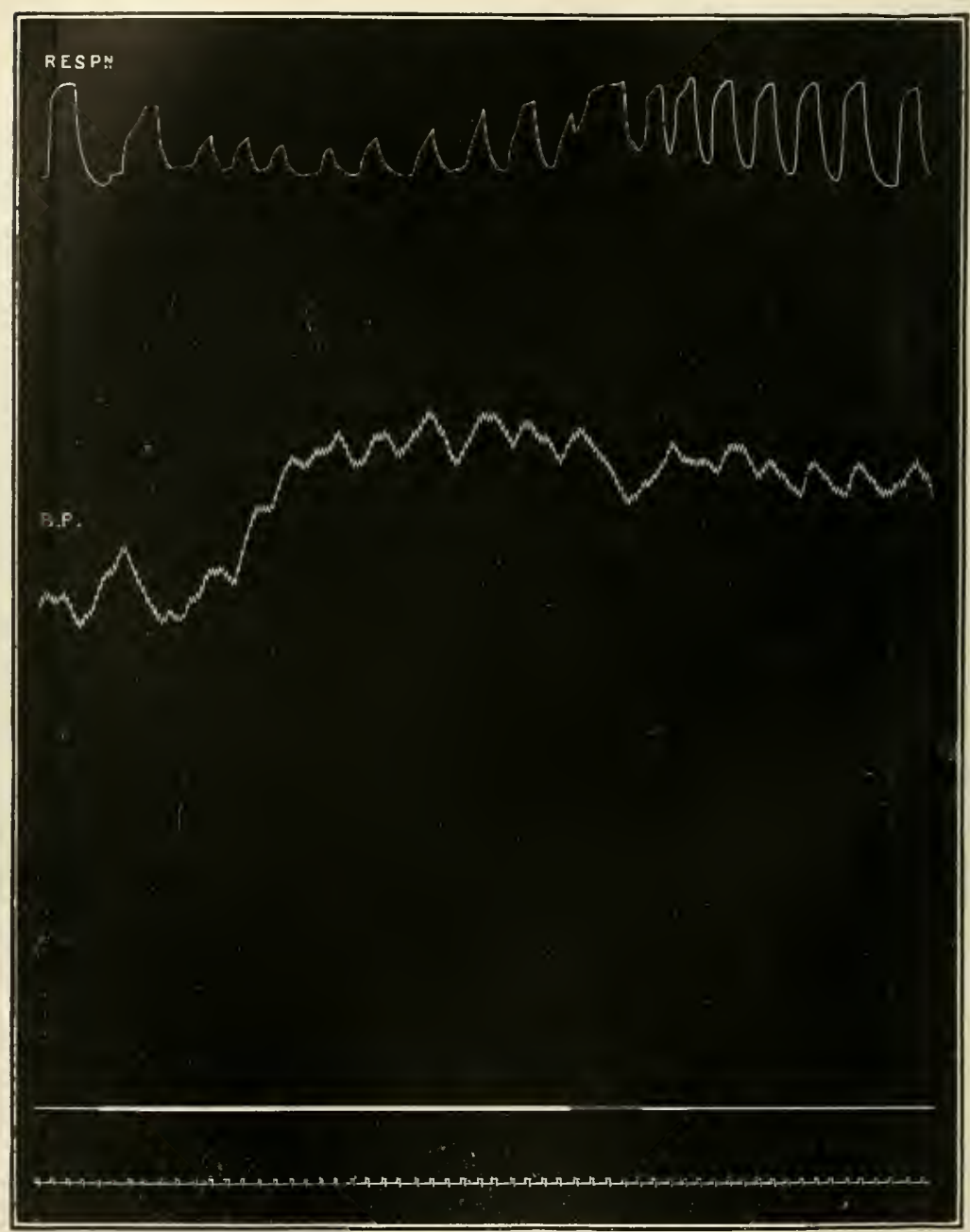

Fig. 142.--Stimlation of the Central End of the Left Vagus in a Rabits.

duces an effect upon the curve. Fig. 142 shows such a result, where the effect is seen to persist for some time after stimulation ceased. If the strength of stimulation be increased convulsive movements are produced which send up the blood pressure; but at the same time 
marked if the dose be at all large that the mercury may be driven out of the manometer. In the figure reproduced it is seen that the pressure rose gradually from a mean pressure of $84 \mathrm{~mm}$. $\mathrm{Hg}$ to one of $136 \mathrm{~mm}$., and from that point it gradually sank, though some minutes later it was still distinctly higher than at the start. At the commencement of the tracing there are some irregularities which were due to alterations in the respiratory rhythm. This rise is due to a marked constriction of the arterioles. A similar result, though not to so marked an extent, is produced by an injection of a watery extract of the medullary portion of the supra-renal gland. On the other hand an extract of the cortex produces practically no effect upon the blood pressure.

7. The effect of asphyxia.-The experiment may be conveniently concluded by killing the animal by asphyxia. This is effected by compressing the trachea by clip forceps or a ligature. If the animal be under the influence of curare, stopping the artificial respiration is sufficient.

Death by asphyxia is described as occurring in three stages, producing typical results in the blood-pressure tracing. The first stage, lasting from $\mathrm{A}$ to $\mathrm{B}$ (pl. 2), is the stage of dyspnœa. The blood pressure gradually rises and the animal makes deep inspiratory efforts, each being sustained for a time, and expiration is rapidly followed by a fresh forcible inspiration. The blood pressure shows variations corresponding to these respirations. This stage is followed after a varying time by the second - the stage of convulsions. (From B to c.)

In this stage the animal passes into a rapid series of convulsive struggles, as seen upon the respiratory tracing; and with each struggle the blood pressure rises considerably. The mean blood pressure reaches its greatest height during this stage. The third and last stage is characterised by a gradual weakening and slowing of the heart and a fall in blood pressure. The animal becomes quiet, and only a few respirations are attempted. No other muscular efforts are made. The heart gradually ceases to beat and the animal dies.

Note that at death the blood pressure has not reached the zero abscissa, but lies $8 \mathrm{~mm}$. above it. If the aorta be cut into, and the blood allowed to escape, the manometer falls to zero pressure. The pressure at death is not zero pressure, because the blood is contained in a closed system of tubes, which it overdistends, with a uniform pressure throughout, and this pressure is spoken of as the mean general pressure. During life the blood is very differently distributed, the arteries being overfilled, and therefore the arterial pressure is much above the mean general pressure. The large veins, on the other hand, are not distended to the full amount, and the pressure is below that of the mean general pressure. 


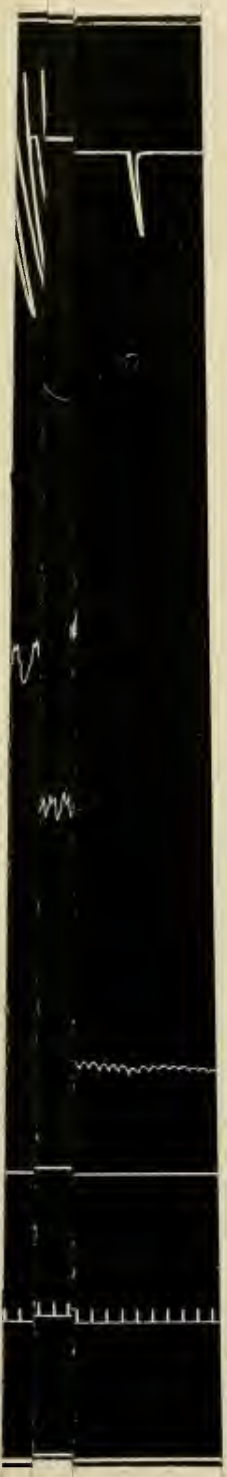

iraker e convulsive 


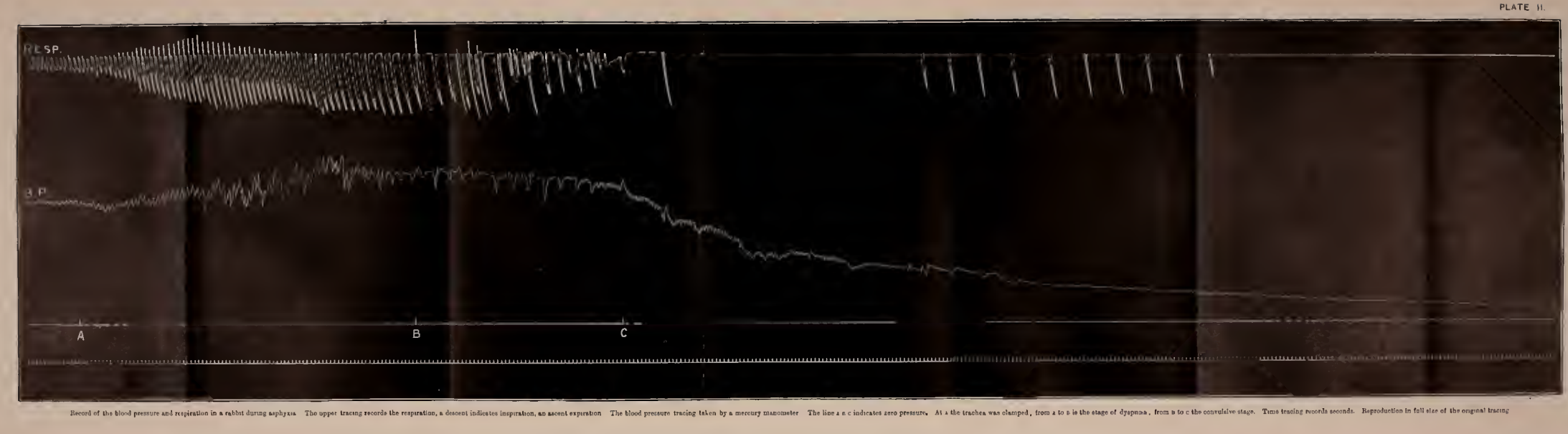




\section{OTHER METHODS OF RECORDING BLOOD PRESSURE}

We have had occasion to note that the mercury manometer is in certain respects deficient, orving to the great inertia of its moving parts. A small force, therefore, which lasts a short time can only produce a very minute effect, and this will be maslied $\mathrm{by}$ any larger effects occurring simultaneously. Even variations in pressure of as great amount as those produced by a lieart beat are only recorded after a considerable delay, so that before the inercury has had time to reach the level which represents the highest pressure attained that pressure has ceased to act, and is again falling.

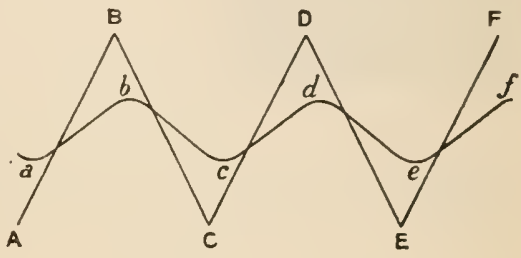

Fig. 144. Fig. 144 illustrates this point.

Suppose the line A B C D to represent the actual changes in pressure in a liquid where vertical ordinates represent pressures, and horizontal ordinates time. If the changes take place very slowly then the mercury manometer is able to register them as the line $\mathrm{A} \mathrm{B} \mathrm{CD}$ with absolute accuracy. If, on the other hand, the changes are carried out rapidly, the record obtained is very different, and something like the line $a b c d$. The mean value is the same in both cases, but the amplitude of the record is much less; the highest and lowest pressures are not recorded, and, moreover, there is a delay: the highest points of the curve $b$ and $d$ lie more to the right, and therefore later than the in. stants of time at which the highest pressures actually occurred. The apices also are rounded instead of sharp.

'To obtain, therefore, a true record of the changes of fluid pressure in an artery, we see that one of the first necessities is to choose apparatus in which the moving parts possess the least possible inertia. One of the first means that suggests itself is that we should record changes in diameter of the artery whose pressure we wish to record. This will give us a measure of the variations of blood pressure in the artery, because the artery wall being elastic, the lateral pressure upon the wall causes a stretch-

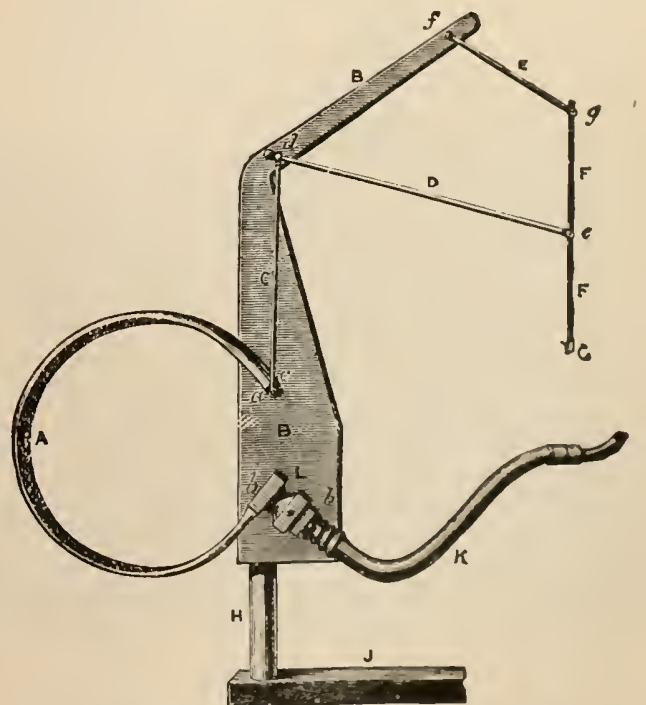

Fig. 145.-Fick's C-Sinisg Mavometer. (Yeo.) ing of the wall in proportion to that pressure. Having recorded the variations we can subseruently calibrate them by recording the diameters of the same piece of artery under 
constant pressures. This method gives far more accurate results than themercury manometer, but possesses certain disadvantages.

There are two instruments which are employed for the purpose. The

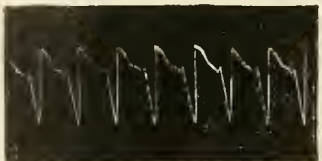
first of these is Fick's spring kymograph, fig. 145. It consists of a C-spring, A, made of thin strips of metal united at their edges. If the pressure in the space thus caused be increased the spring opens. out. It possesses little inertia, and therefore responds quickly to changes of pressure. Fig. 146 gives. a small piece of tracing taken with this manonieter.

Fig. 146.

Recently a modification of this kymograph by $\mathrm{r}$. Basch has been introdnced in which the $\mathrm{C}$-spring: is reduced to the smallest size. It gives rery excellent results.

The other instrument is Fürthle's rubber mancmeter.

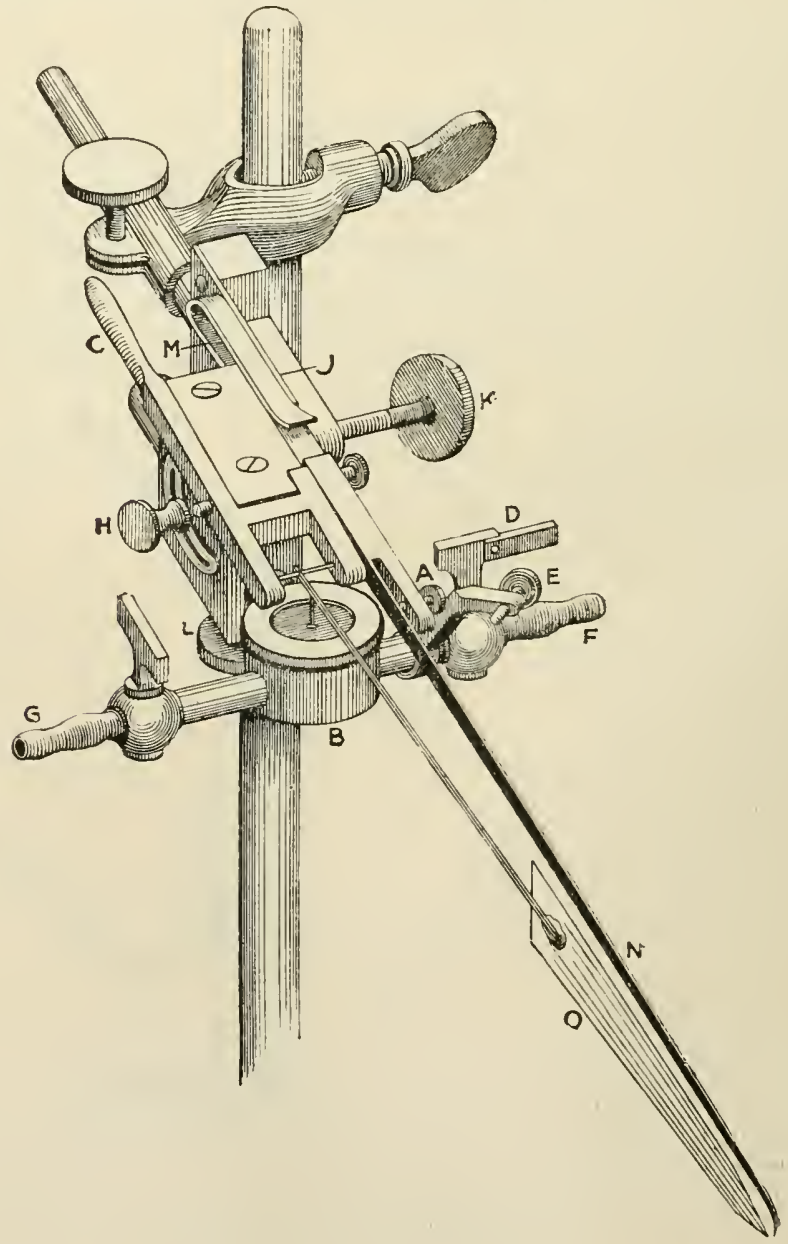

Fig. 147.-Hünthle's Maxonetel:. 
are not so conspicuous as in fig. 135. This is due to the smaller magnification obtained in this instance. The heart beats are, however, more conspicuous and show greater oscillations than those caused by respiration. If measured they are seen to be $1 \frac{1}{2} \mathrm{~mm}$. high in a total blood pressure measured by $16 \frac{1}{2} \mathrm{~mm}$., i.e. the variation caused by each beat is ${ }_{11}$ th of the mean pressure. If we make similar measurements in the trace yielded by the mercury manometer we find each beat is about $1 \mathrm{~mm}$. high in $75 \mathrm{~mm}$. mean pressure, i.c. the variation in pressure recorded as due to each beat is $\frac{1}{7}$, th of the mean pressure. Hence in this particular experiment the Hürthle manometer was seven times more sensitive in recording rapid variations of pressure than the mercury manometer. Tracing II of fig. 148 is a further illustration of the different effects of normal and artificial respiration upon the blood pressure. This piece of tracing also demonstrates the fact that in artificial respiration a fall of pressure is produced by inflation of the lungs. The production of this result we have already explained on p. 174.

In fig. 149 is reproduced a tracing of the effect of stimulation of the peripheral end of the vagus of sufficient strength to cause slowing

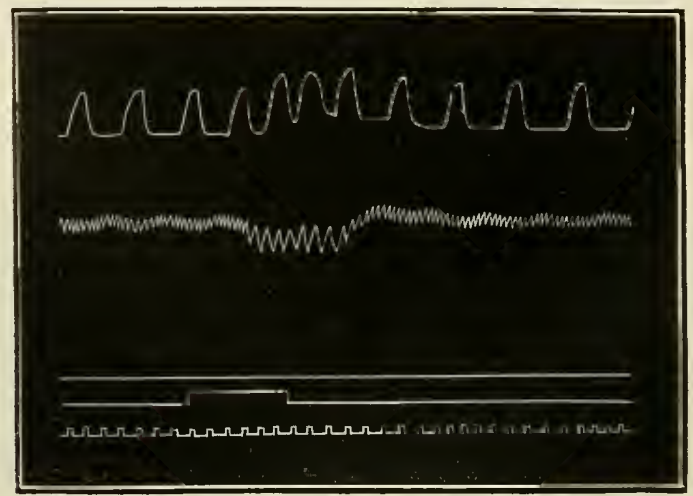

Fig. 149. - Strmulation of the Peripheral Exd of the Vagets. Tiabist. Hürthle Manometer. The Upper Horizontal Line marks Zero Pressure.

of the beat but not stoppage. This should be contrasted with those given in figs. 140 and 141.

The most interesting results to be gained by the use of this instrument are, however, to be seen when the record is more extended by making the movement of the blackened surface more rapid. Such a record is given in fig. 150. It shows on the blood-pressure tracing practically the same variations as those obtained in a sphygmogram (see fig. 131, p. 167). Thus from $a$ to $b$ is the rise in pressure due to 
the sudden propulsion of blood into the aorta ; at $c$ is the predicrotic wave, and at $d$ the dicrotic wave.

This piece of tracing must also be compared with that given in fig. 146, which was obtained from a dog, using Fick's kymograph.

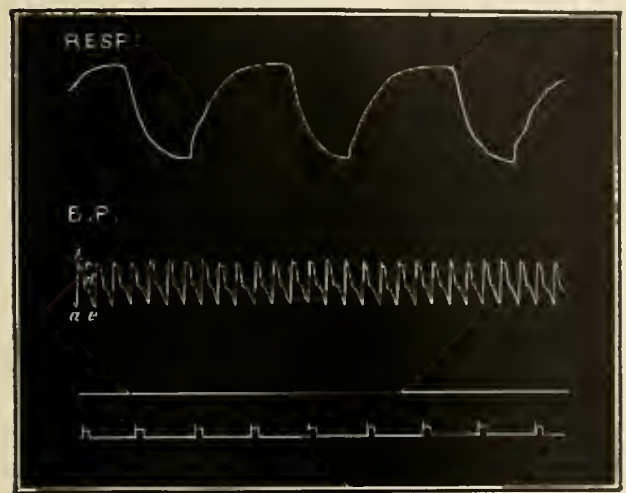

Fig. 150.-Nordal Blood Pressure and Respiration. Raвbit. Hёrthle Manometer.

Both tracings show precisely the same points, and further show that by employing these instruments we are able to record even the smallest and most transient variations in pressure.

In connection with the consideration of the blood flow the student should examine the construction of the following piece of apparatus whose aim is to enable us to determine the rate of flow of the blood along a vessel. Ludwig's stromuhr (fig. 151) consists of two glass bulbs, $\mathrm{A}$ and $\mathrm{B}$, which communicate with each other above, and below are fitted into a circular brass base, $\mathrm{P}$, revolving on a lower frame, E. Through this frame are two ehannels, opening respectively at $\mathrm{C}$ and $\mathrm{D}$. By means of two stops the revolving disc $P$ is checked in the two positions, so that either A or $\mathrm{B}$ can be brought into communication with the tube at $c$. The opposite bulb then opens out at $\mathrm{D}$. The method of employing the apparatus is to fill the bulb $\mathrm{A}$ with oil and the bulb B with defibrinated blood. The tube $c$ is then connected to the central end of the artery the rate of flow of the blood along which we wish to determine, and $\mathrm{D}$ is tied into its peripheral end. At an instant which is recorded the blood from the artery is allowed to flow into $\mathrm{A}$. The oil rises on the surface of the blood, and the upper oritice of the bulbs being closed it forces the defibrinated blood from $B$ into the peripheral end of the vessel. As soon as A is filled with blood the bulbs are rapidly rotated, so that the bulb B comes to occupy that position previously held by $A$. The bulb is now con. tains the oil, and more blood entering it from the artery, the oil is once more 
lisplaced and the blood previously entering $\mathrm{A}$ is sent into the peripheral end of the vessel. When $\mathrm{B}$ becomes full the apparatus is again rapidly rotated, and the operation repeated several times, when the experiment is stopped and the total time occupied observed.

If now we know, by previous measurement, the capacity of the bulbs and the number of times they have been rotated, we know the total volume of blood that has left the artery during the experiment. Suppose this to be V c.c., and the whole time of the experiment to be t. seconds. Then in 1 sec. a volume of $V / t$ e.c. left the artery. The lumen of the artery is next measured. Suppose this to be $a \mathrm{sq}$. cm., and that the velocity of the blood is $v \mathrm{~cm}$. per sec. Then the volume of blood leaving the ressel in 1 sec. would be $a . v$ c.c.
Hence
$a \cdot v=\mathrm{T} / t$,
and

$$
\mathrm{v}=\frac{\mathrm{V}}{a \cdot t} .
$$

Thus from a measurement of the area $a$ of the lumen of the ressel, and the volume $\mathrm{V}$ issuing in a time $t$, we can, by the above formula, determine $v$, the velocity of the blood flow, along the artery. 


\section{CHAPTER XVIII}

\section{THE KIDNEY. DEMONSTRATION OF AN ONCOMETER EXPERIMENT}

Is investigating the mode of action of any organ or part of the body one of the first things we require to know is how its blood flow is modified under different conditions, in order that we may be able to correlate these changes with other functional activities simultaneously observed. There are several methods open to us by means of which we may observe changes in vascularity of an organ. In many eases simple inspection by revealing differences in colour is able to show us that there is more or less blood in the organ, but this method can only show us gross changes. A second plan is to measure the quantity of blood issuing from the organ, variations in the rate of ontflow giving a measure of the amount of blood passing through the organ. This is one of the most satisfactory methods if we are working upon an exeised organ and cireulating defibrinated blood through it by tying a cannula into its main artery. The third and the most valuable is that known as the plethysmographic method. Here the changres in volume are directly measured by confining the organ in some enclosed space and then recording the amount of air or of thi displaced from this space as the organ expands or contracts.

The general plan adopted in such an experiment may be best illustrated by one upon the kidney.

The original form of apparatus, as invented by lioy for experiments upon the kidney, consists of a metal box, in which the kidney is placed, enlled an oncometer (bullk measurer), and a piece of recording apparatus termeil an oncograph (bulk recorder). The form of the oncometer is shown in tig. 152. and a diagran of its general principle in fig. 153. It eonsists of an ellipsoidal metal box nade to open by a hinge. Each half consists of at donble metal box (oc and Ic, fig. 15i3), the one fitting tightly within the other. 'To prepare it for use each inner half is removed and a sheet of sheej's peritoneal membrume, $\mathrm{x}$, is fitted over it, and its edges gummed down to the onter surface of the eapsule, to make an air-tight joint. The membrane is titted on very loosely. and thus allows free movement inwards and ontwards. The imer box is then replaced in the onter, to which it is tighty clamped by the serew s. Both sides are now filled with oil, so as to fill $m$ the spaces $A$ and $n$ between the peritoneal membrane and the inner cases, the membrane being raised meanwhile so that when the instrument is closed there is suflicient spuce left 
inside to receive the kidney. The one orifice is then tightly closed by the cork $\mathbf{B}$, and a special two-way cannula fitted into the other. The instrument

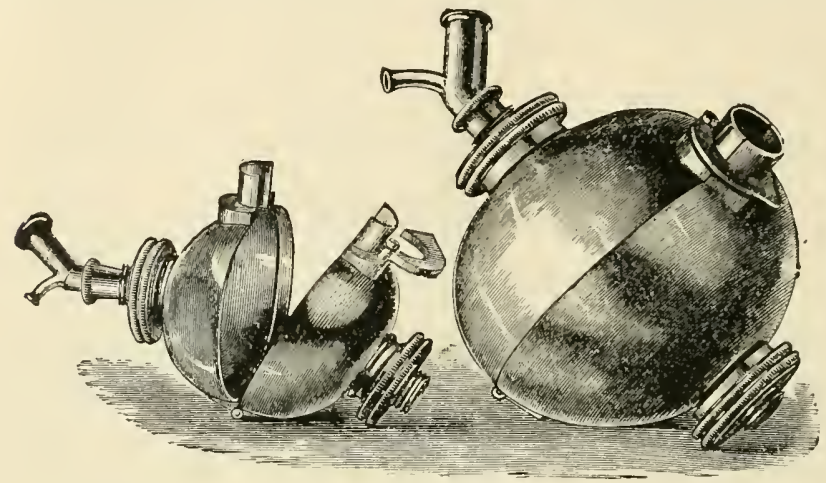

INST, CO. LTD, CAMB.

Fig. 152.-Two Sizes of Roy's Kinney Oxconeteri.

is then placed in an air-bath to raise its temperature to body temperature. When the kidney is inserted between the two halves, the peritoneal membrane,

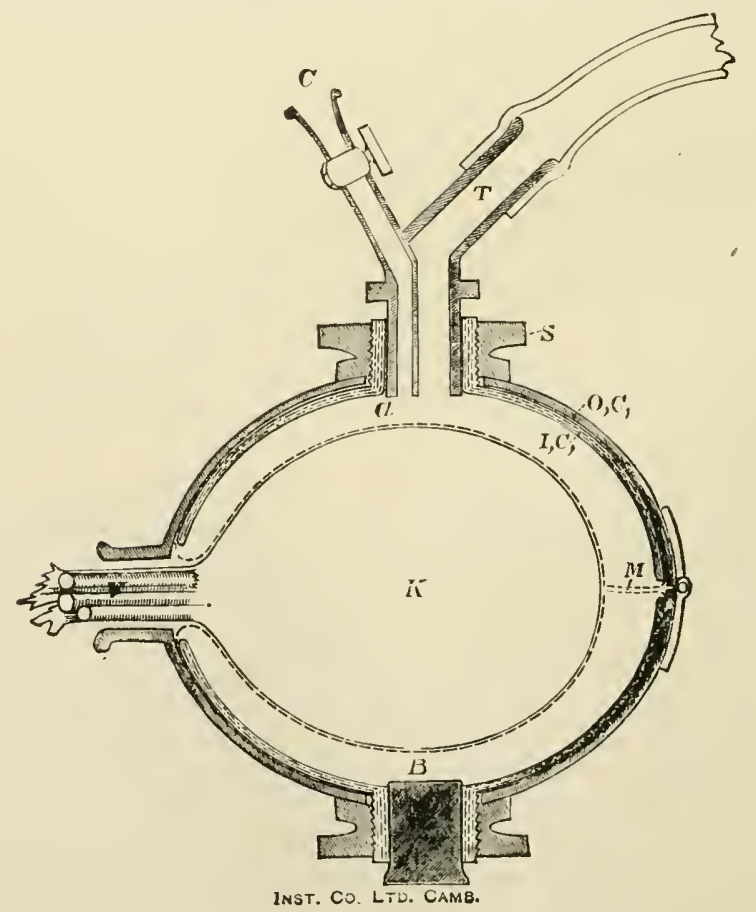

Fig. 153.-To Illustrate the Principle of Riv's Onconeteir. 
being very flexible, adapts itself to the kidney surface, so that practically the kidney is inserted into an oil-tight box filled with oil. The oncograph, fig. 154, consists of a metal box whose upper surface is elosed by a sheet of peritoneal membrane fitted on very loosely, so that it can move fairly freely. On this rests a small cylinder of ebonite with its lower end closed, and to this is attached a light rertical rod which moves a horizontal recording lever. 'The interior of the oncograph is connected hy a piece of rubber tubing with the

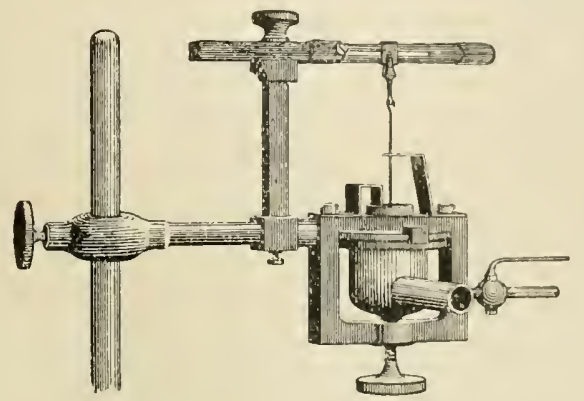

Fig. 151.-The Oxcograph.

interior of one side of the oncometer by the tulie $\mathrm{T}$, figr. 15:3. The whole oneograph and tube connection is filled with oil. Thus arranged any expransion of the kidney drives out oil from the oncometer into the oncograpl, the peritoneal membrane of which is bulged upwards, and lifts the vulcanite cup. and thus the lever of the oncograph. In this way the variations in volume are recorded

A very simple but effective method is to employ an air oncometer made after the principle of that used by Schäfer and Hoore in their experiments upon the spleen. A convenient form is shown in fig. 15.). It may be made

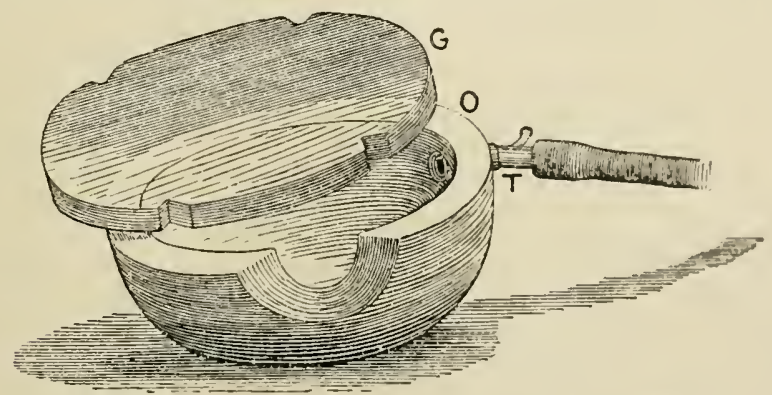

Fig. 155. - Ax AIR Oxconeteli Fon THE hinNi:.

either of plaster of Paris, which is thoroughly soaked in melted hard paraftin, or of wood, which is then thoronglyly varnished. The figure slows its general shape. It has a lid made either of thick plate griss or of wood. A depression is ent away in one wall through which the kidney ressels and ureter may pass when the kidney is placed within the hox. The wholo is made air-tight by filling the hole in the side with pieces of wool soaked in a stiff mixture of vaseline and hard paraflin, so that the kidney ressels are 
not constricted. The junction with the lid is also made air-tight by vaseline. Piercing one side is a hole into which a tube fits tightly, and this is connected by rubber tubing of small bore to a tambonr (fig. 35). As the kidney expands air is driven out of the plethysmograph into the tambour, the lever of which therefore rises.

\section{DEMONSTRATION OF THE CHANGES IN VOLUME OF THE KIDNEY}

The apparatus is first set up as for taking a blood-pressure tracing, and a tambour is also fitted up to record vertically above the blood-pressure tracing. Care is taken that all the writing points record vertically above each other.

A dog which has not been fed for the previous twelve hours is put under ether and then a dose of morphia is injected, 1 c.c. of a 1 per cent. solution of the hydrochlorate per kilo of body-weight. A median incision in the neck is made, the external jugular isolater for about $1 \frac{1}{2}$ inches, and a simple cannula inserted and tied in. The carotid artery is next exposed, and a cannula tied in it as described on p. 170. The kidney is next exposed. This may be done by a lateral incision in the lumbar region and the kidney isolated from the peritonem without opening the abdominal cavity if possible, though this will be found of some difficulty in the dog. There are usually several small vessels which enter directly into the cortex of the kidney. These have to be divided; but each must be ligatured before doing so in order to avoid hemorrhage into the oncometer. When the kidney is thoronghly freed it may be placed in the oncometer.

The other method, which has the great advantage that the kidney is more fully exposed, is to reach it by opening the abdominal cavity from the front. If care be taken to protect the exposed viscera from cooling, this method inay be safely adopted. A longitudinal median incision is made through the skin, starting just below the xiphisternum for about three or four inches. A transverse incision through the skin at the level of the last rib is now made, from the mid-line down to the rib on the left side. The muscles are next cut through, one at a time, along this line-first the external oblique, then the internal oblique, then the transversalis, and finally the rectus. Care is taken that the peritoneum is not opened until all bleeding has been stopped. As each ressel is cut through it is canght up on Spencer Wells' forceps and ligatured. When all bleeding has been arrested the abdominal cavity is opened by cutting through the peritonemm along the incisions made through the muscles and in the mid-line. The intestines are pulled well over to the riglst side and protected by covering with a thick layer of cotton wool which has been warmed in front of a fire. The kidney is then exposed and the peritoneum over it is torn throngh, any bleeding point being ligatured. Care is taken to handle the kidney as little as possible, and it must not be allowed to become cooled. Having thoroughly isolated the kidney, especially where the vessels leave it, it is placed in the oncometer. This has previously been prepared by warming it and then placing a few layers of cotton wool containing a plentiful amount of the vaseline and paraftin mixture at the bottom of the notch in the side wall. The kidney is placed in the oncometer, so that the vessels and ureter lie on the vaseline wool in the notch for that purpose. Strips of wool soaked in the vaseline mixture are now packed, so as to lie between the kidney and the notch, and others to fill up the notch. The whole success of the experiment depends upon the careful packing at this stage. 'The notch is to be exactly filled and not overfilled, so that when the lid is adjusted there is no pressure on the kidney ressels. Before putting on the lid the upper edge of the oncometer is thoroughly covered with vaseline 
mixture and then the lid is rubbed down into close contact with the edge. The lateral tube is now connected by thick-walled rubber tubing to the recording tambour. In the rubber tubing is inserted a T-piece, the lateral orifice being closed by a rubber tube clamped by a spring clip. The tambon1. now shows the oscillations due to the heart beats. The oncometer must now be tested to see if it be air-tight by blowing in a little air through the $T$-piece into oncometer and tambour. The tambour lever is raised and the pressure of air inside is greater than atmospheric pressure. If the tambour lever fall there is a leak, and the oncometer must be reopened and the packing move thoroughly carried ont. If the tambour lever remain at the same mean level the experiment may be proceeded with. The whole abdomen is thoronghly covered up with warmed layers of dry cotton wool, the abdominal wall having been previously drawn together by sutures.

The cannula in the carotid is now connected to the mercury manometer and the writing points brought to the recording surface. The procedure of the remainder of the experiment can be varied in different ways according to circumstances.

The following five paragraphs give the course of an experiment which will show the main facts to be gained by the method. The results obtained are examined in the succeeding pages :-

1. Having brought the air pressure inside the oncometer to atmospheric pressure, by opening for an instant the clip on the lateral pass of the T-piece, take a piece of normal tracing.

2. Inject 1 c.c. of a 4 per cent. solution of caffeine citrate in 1 per cent $\mathrm{NaCl}$. To do this fill the cannula and rubber tubing in the external jugular vein with the solution by means of a pipette drawn out to a fine point. Fill a 1 c.c. pipette with the solution and attach to the cannula by rubber tubing. Take a short piece of normal tracing and removing the bull-dog forceps from the central end of the vein, at a given signal blow in 1 c.c. of the solution. Replace the clamp on the vein. The instant of injection is marked on the tracing by means of a signal. If this dose be not sufficient repeat with an increased dose until a typical effect is produced.

3. After complete recovery from the caffeine citrate inject 5 c.c. of a 0.1 per cent. solution of digitalin in 1 per cent. $\mathrm{NaCl}$. Previously remove the caffeine solution still in the cannula by sucking it up in a pipette drawn out to a fine tube, which can pass down the cannuli ; then wash out with normal saline, remove the saline, and fill with the digitalin solution. Attach a pipette containing 5 c.c. of the new solution and inject at a given instant.

4. Inject 1 c.c. of a solution of neurine made by adding one drop of a 25 per cent. solution of neurine to 5 c.c. of 1 per cent. NaCl. The cannula is to be washed out and filled with the solution as in 3.

5. To complete the experiment, record the kidney changes during asphyzia. Dissect out the trachea, open it widely, and while a tracing is being recorded suddenly plug it tightly with cotton wool soaked in water. 
Typical tracings obtained in such a demonstration are given in the following figures. It is seen that as a rule the trace follows

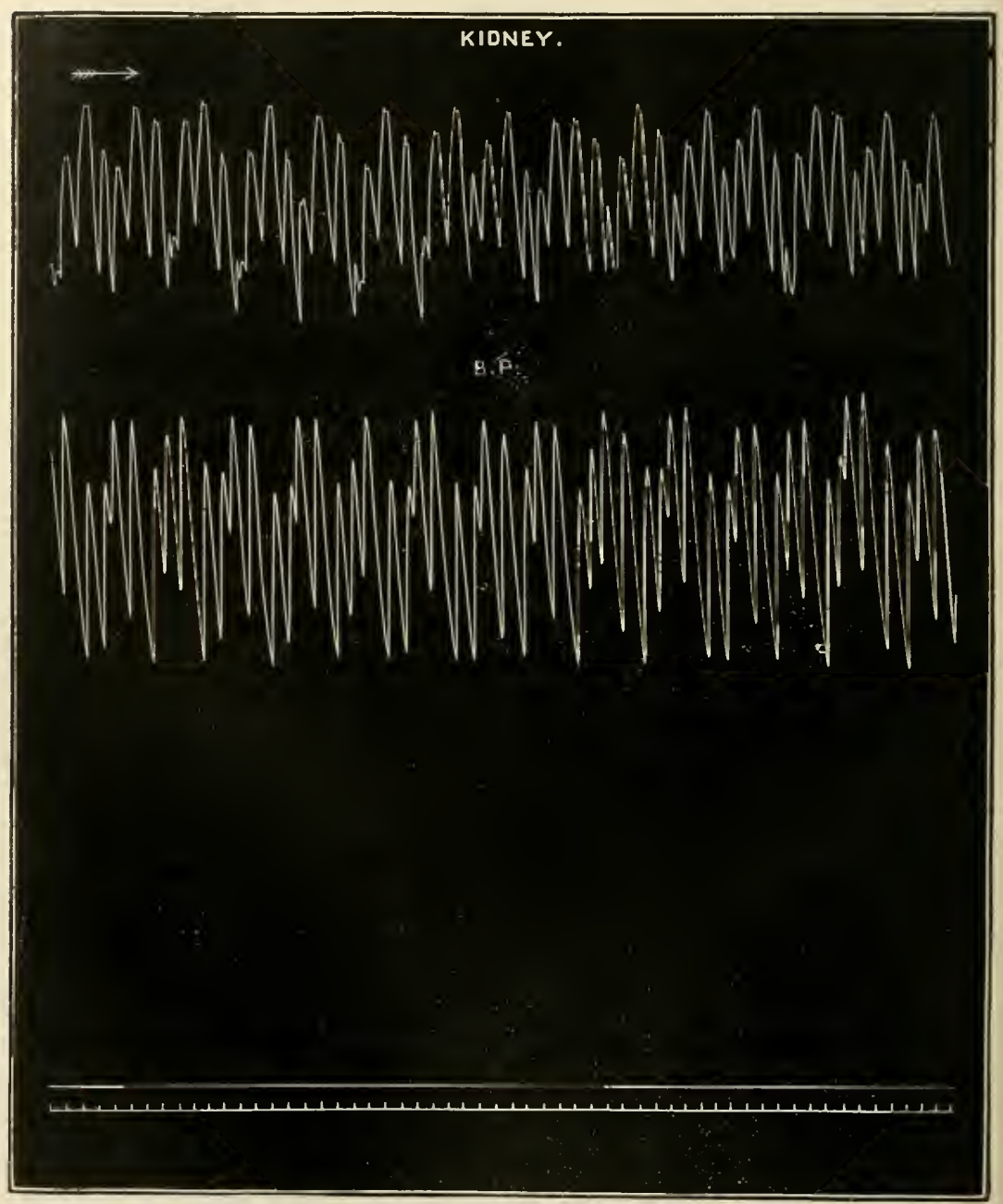

Fig. 156. - Simultaneous Tracing of the Volume Changes of the Kidney and of the Carotid Blood Pressure in a Dog. Thae Tracing Seconds. The Hortzontal Line jarks Zero Blood Pressure.

exactly the curve of blood pressure simultaneously recorded. The variations in volume with each heart beat are well marked, and the 
rise and fall of blood pressure during respiration are represented by an increase and decrease of volume of the kidney. This is exactly the result we should expect to obtain if the vessels follow passively any variations of blood pressure, and this is the result during ordinary conditions when the kidney is at rest. Comparing fig. 156 with fig. 157 , both of which were yielded by the same animal, we see how closely volume changes follow blood pressure changes. Fig. 156 was obtained at the commencement of the experiment, and is therefore to be taken as especially typical of the volume changes. Fig. 157 was obtained later, and with a less magnification. The respiratory movements altered in character as this tracing was being recorded. There occurred an increase in kidney volume and a fall in blood pressure, so that the fall in blood pressure is chiefly to be explained as due to dilatation of blood vessels in which the kidney took part. The alteration in volume closely follows that of the blood pressure. In watching the two being recorded it is very obvious how the rise of the oncometer lever precedes by a quite appreciable interval the rise of the manometer float. 'This difference is purely instrumental in origin, the kidney rise being recorded by apparatus having very little inertia, whereas the inertia of the mercury manometer is great.

Changes in volume of the kidney may be brought about in either of two ways :-

(i.) Passively, in which case the increase and decrease of volume follow proportionately a rise and fall of blood pressure. This is the case, as we have seen, with the ordinary kidney trace, where the rapid undulations in the blood pressure, due to heart beats, and the slower, due to respiratory effects, are exactly reproduced in the kidney oncogram.

(ii.) Actively, in which case changes in volume of the kidney are brought about independently of changes of blood pressure or force of heart beat, and may even work against these. Thus an active constriction of the kidney vessels leads to a diminution of kidney volume and a rise in blood pressure. These changes in kidney volume are best studied by experiments which locally influence the kidney vessels. Of these changes some of the best known are those produced by drugs which are known to influence the secretion of urine. They are also of greatest interest because they teach us at the same time something of the way in which the kidney works when it is called upon to secrete more actively.

Action of Caffeine.-In fig. 158 is reproduced the effect of an injection of 2 c.c. of a 4 per cent. solution of enffeine citrate in normal saline directly into the external jugular vein. After a period of delay it is seen that the blood pressure falls, the variations in pressure with each heart beat become less, then increase, and the blood 
pressure soon rises again and returns to its initial value. In the case of the kidney trace there is the same delay before any effect is produced, and then a little later, after the blood pressure begins to

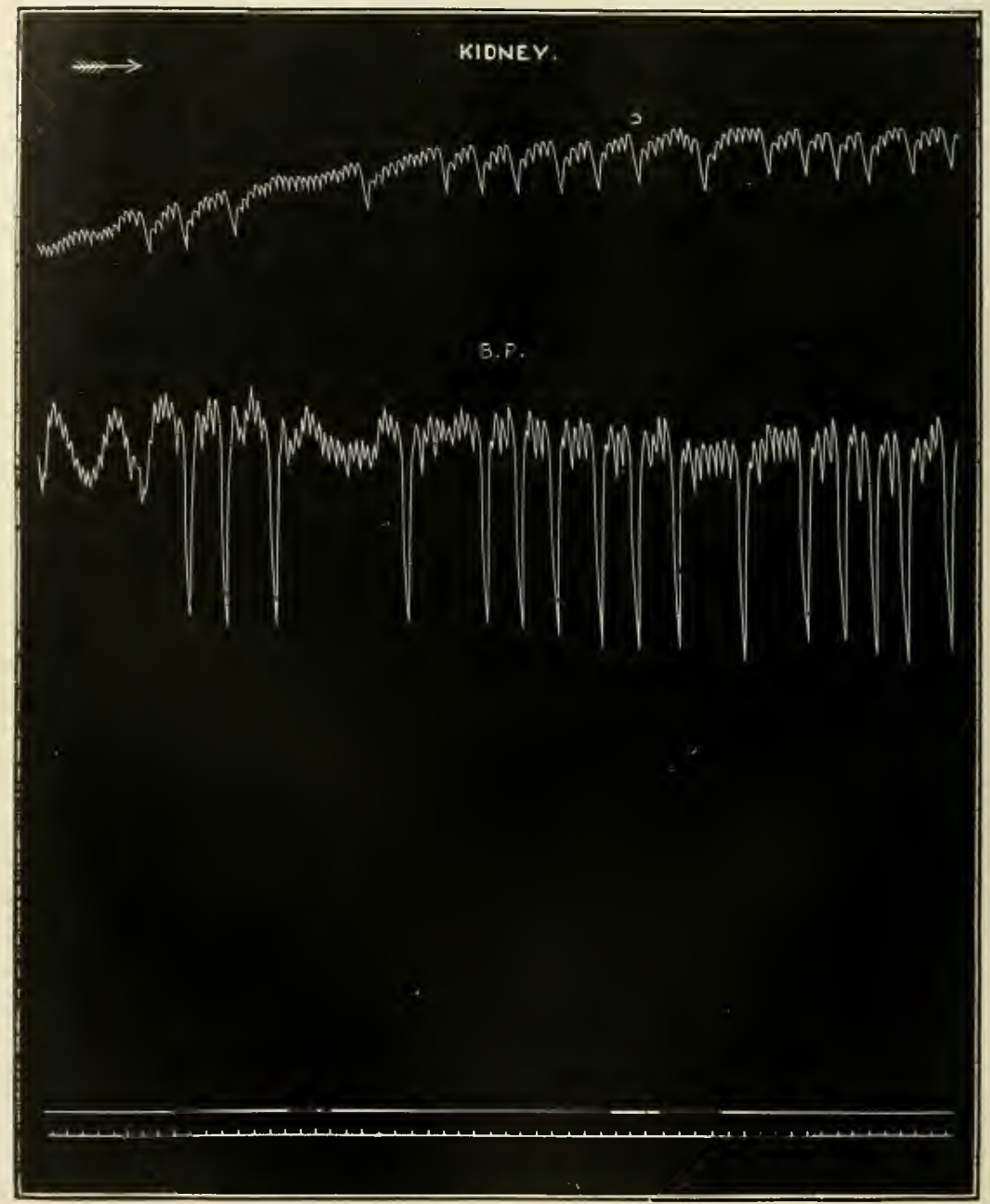

Fig. 157.-Kidney Volume and Blood Pressure in a Dog.

alter, the kidney volume falls and remains less for some time, finally expanding, often to a much higher volume than before the injection. Finally, after a variable time it once more returns to its initial state. The explanation of these changes in volume of the kidney may be 
twofold, i.e. either passive or active. The fall in blood pressure must produce a diminution of the kidney volume unless it be over-compensated by another volume change in the reverse direction. The fall of volume that actually occurs is partly to be explained by the fall in blood pressure, but not entirely, for the fall in blood pressure usually precedes by a definite interval the fall in the kidney trace, and secondly the blood pressure attains its original height long before the kidney begins to expand. The main cause producing the diminution of the kidney volume is therefore active, and due to constriction of its

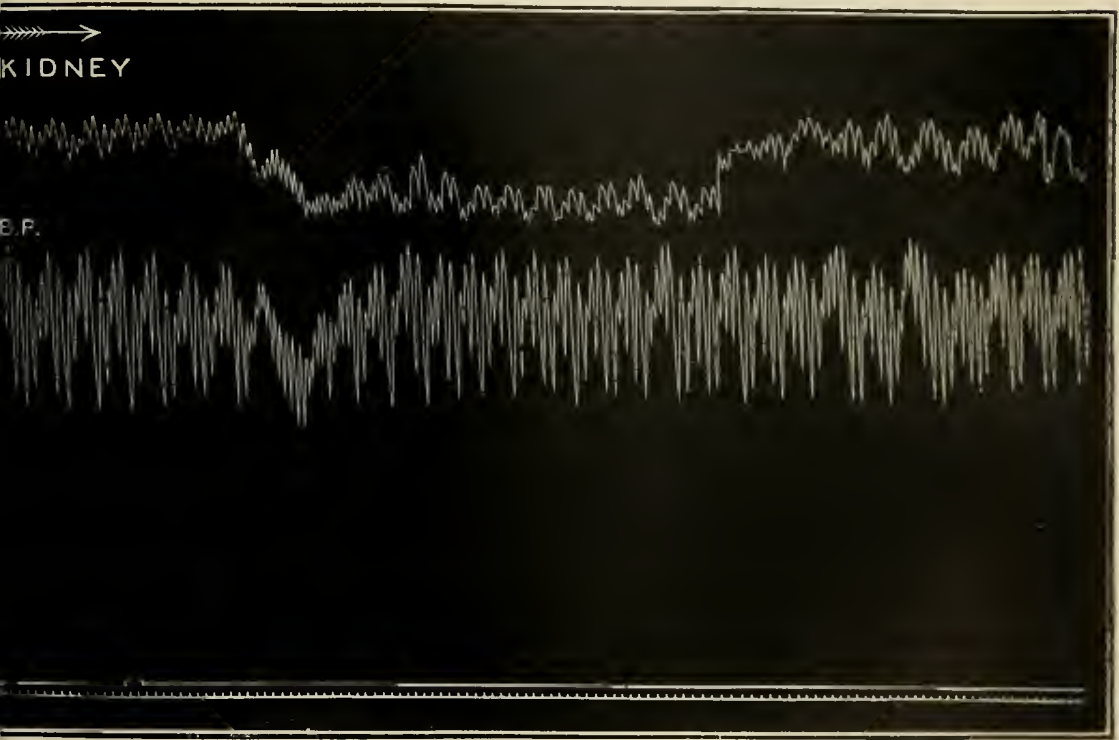

Fig. 158.-Effect of Caffeine upon the Kidney Volume ani Blood Pressure. Tracing reduced to Half Size.

blood vessels. This constriction ultimately yields, and is followed by a dilatation lasting a still longer time, and then the kidney returns to its initial state. When the rate of secretion of the urine is recorded at the same time as the kidney changes it is found that this rate varies and accurately follows the changes in volume of the kidney. When the kidney vessels contract the rate of secretion drops, and in the second stage, the period of relaxation, the secretion is accelerated, and finally, as the kidney regains its original state, the rate of secretion returns to that observed at the commeneement of the experiment. The fall in blood pressure produced in this experiment is due to the 
direct action of the drug upon the heart. This follows from the experiment already demonstrated, in which the force of the heart beats was directly studied (fig. 113, p. 143).

The changes of rate of flow studied in association with the changes in volume are of interest when connected with the observations which show that rate of secretion of urine depends rather upon rate of flow of blood through the kidney than upon changes in blood pressurethough these must of course act secondarily. Increase in urine flow being associated with a dilatation of the kidney vessels directly confirms this view.

Action of Digitalin.---The effect on the blood pressure is a fall of short duration followed by a rise of much longer duration. During the rise the rate of heart beat is slower, but each beat is more forcible. The changes in the kidney tracing are not synchronous with those of the blood pressure. There is produced a slow constriction of the vessels which reaches a considerable amount and is very persistent. Gradually relaxation occurs and results in a greater volume than before the injection; but finally there is a return after several minutes to the initial volume. We must note that during the kidney constriction the passive variations in volume due to heart beats are very well marked. These only become masked or obliterated if the constriction be very great; as, for instance, after a very large dose of the drug. The alterations in rate of urine secretion are interesting. During the period of constriction the rate slows considerably, but is accelerated to a certain degree during the subsequent relaxation. Neither the relaxation nor the rate of flow ever attains to the same degree as that observed after an injection of caffeine.

Action of Neurine.-The tracing (fig. 159) is introduced here because it forms a very good demonstration experiment. In the experiment reproduced 0.5 c.c. of a solution, made by adding one drop of a 25 per cent. solution of neurine to 6 c.c. of 1 per cent. $\mathrm{NaCl}$, was injected into the external jugular of a dog. The effect upon the respiration is very striking. After a brief period of delay the animal passes for a short time into a series of short respiratory spasms, next the respiration rapidly slows and ceases, to ultimately recommence, first with some irregularity, then expiration becomes rapid and short and inspiration prolonged, the rhythm remaining regular. Changes in blood pressure closely follow these changes in respiratory rhythm (see fig. 159). At first there is a slight fall, partly due to diminished force and rate of the heart beat. This is followed by a marked rise in pressure as the inspiratory gasps occur, and is accompanied by an acceleration of the heart beat. Then follows a period of fall of pressure in which the heart beat is slowed, and finally as respiration is once more 


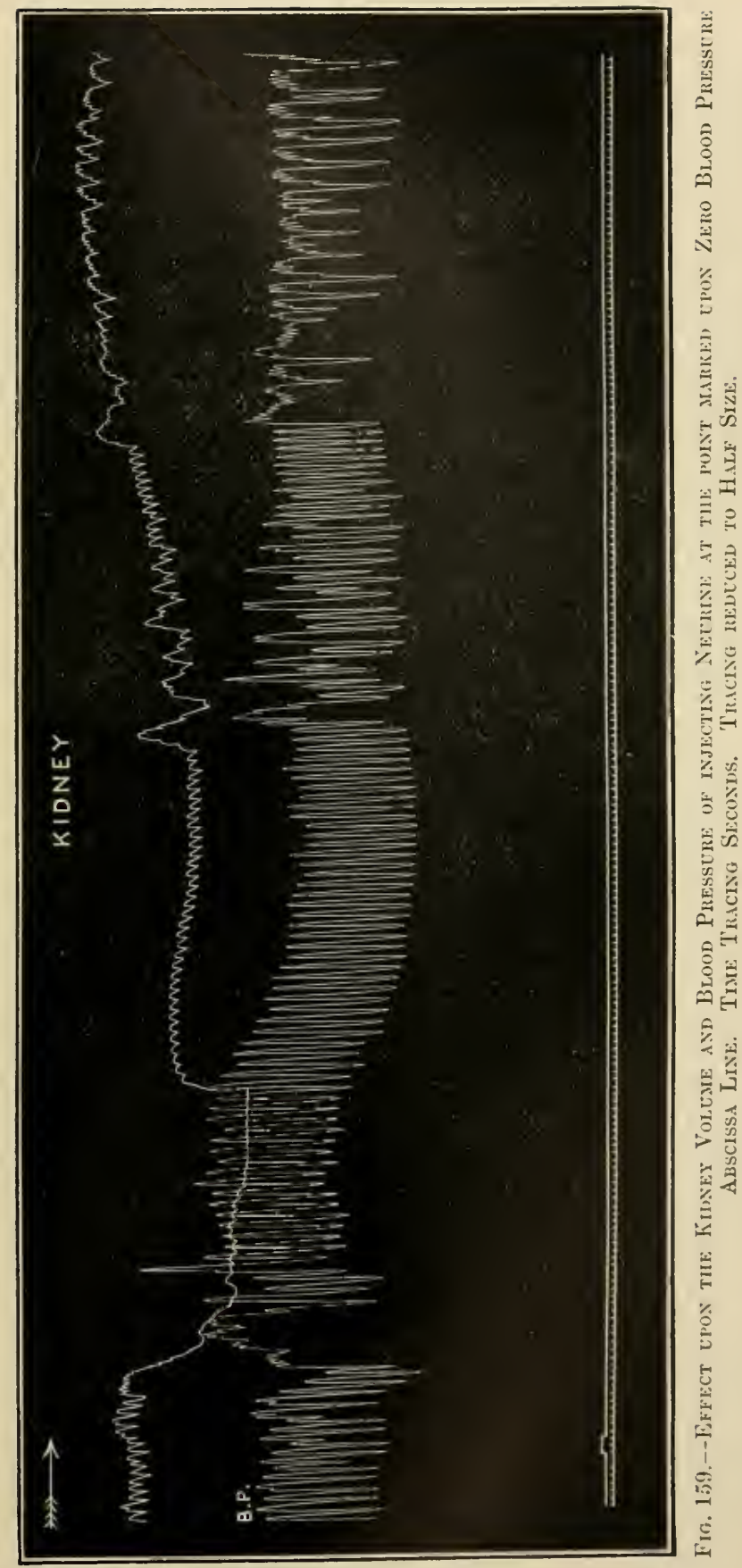


established the rate of heart beat is increased and the mean pressure becomes higher.

The changes of kidney volume do not exactly follow those of the blood pressure, but are to a certain degree independent of them. After a period of delay, which is longer than that necessary to produce changes in the blood pressure, there is a sudden constriction of the kidney vessels, at first very rapid, then more gradual, and finally so marked that the variations due to heart beat are almost obliterated. In this part of the trace a dilatation is to be observed synchronous with the rise of pressure due to the inspiratory spasms. Next follows a very sudden dilatation, and the volume then follows the bloodpressure changes very closely until respiration recommences, when a further very marked dilatation takes place, the volume becomes greater than initially, and then as respiration and blood pressure gradually return to the normal, so the kidney volume recovers, though at a slower rate than the blood-pressure changes. During the marked second dilatation the rate of urinary flow is increased.

\section{THE COURSE OF THE VASO-MOTOR NERVES TO THE KIDNEY}

By means of oncometer experiments the vaso-motor nerves to the kidney have been mapped out. Vaso-constrictor nerves are proved to be present among the particular fibres stimulated if a diminution of the kidney volume result, accompanied by either no change or by a rise of blood pressure. Simultaneous records of the blood pressure changes exclude the possibility of the observed kidney changes being due to variations brought about passively on changes in the blood pressure. These nerves have thus been proved for the dog to leave the cord mainly in the anterior roots of the 11 th, 12 th, and 13 th thoracic nerves, and to a less extent in the 7 th, 8th, and 9 th. Nerve cells, shown by the nicotine method (see p. 133), are found on the course of these fibres, situated in the coliac, mesenteric, or renal ganglia. Vaso-dilators have been found in the 11th, 12th, and 13th anterior roots with ganglion cells in the solar or.renal ganglia. 
Is a previous experiment, p. 168, we saw how we eould record the respiratory rhythm in an animal by the aid of a tambour resting on the thorax or abdomen. This method gives us all that is required so far as the main details of time of inspiration and expiration and rate of breathing are concerned, but we also require some method that will give us a means of recording these same points, and in addition the depth of breathing with some greater accuracy. One method that has been adopted is to make the animal inspire from a large glass vessel which is placed in communication with a tambour, so that changes of pressure inside the vessel cause movements of the tambour lever. Each inspiration eauses a fall in pressure in the glass ehamber, and therefore a fall of the tambour lever. For this method it is not necessary to have the vessel completely closed. The air must also be frequently renewed.

Another method that has been largely employed is to introduce one end of a stiff lever so as to lie between the liver and the under surface of the diaphragm. The lever moves about an axis ncar to this end, so that all that is necessary is to reeord the movements of the free end of the lever. This is usually carried out by attaching it to a writing lever by a fine thread.

In the case of the rabbit we possess a further very convenient and aecurate method, for there exists in this animal a slip of the diaphragm in the anterior mid-line which can be isolated without opening the pleural cavities. A record of the movements of this slip is the method employed in the following demonstration :-

\section{DEMONSTRATION. THE NERVOUS REGULATION OF RESPIRATION}

A rabbit is anæsthetised with ether. By a median incision in the neck: extending for about $1 \frac{1}{2}$ inches above and below the larynx, the two ragi are first isolated and threads passed under them. The superior laryngeal on 
one side is next isolated and a thread passed under it, so that it may readily be lifted up when required. The nerve is easily found as a branch of the vagus rumning off at right angles to the trunk towards the mid-line at the level of the larynx. It usually passes beneath the carotid artery. 'The glossopharyngeal of one side is separated in a similar manner. It is found by tracing the vagus up to the base of the skull. It is found running from the vagus deep down opposite the angle of the jaw, and there lies to the outer side of the carotid. It runs forwards to disappear under the posterior edge of the mylo-hyoid.

A median longitudinal incision about 2 inches in length is next made with the xiphoid cartilage as its central point. All the tissues are then cnt throngh down to the sternum and cartilage, and any bleeding is stopped. The abdominal cavity is opened at the tip of the ensiform cartilage, and the two muscular strips of the diaphragm isolated on either side. The cartilage is then cut across, care being taken not to injure the attachment of slips to its under surface nor the blood vessels to the slips, which leave the lower surface of the sternum at about its junction with the cartilage. A sharp hook made of a bent pin is then passed through the edge of the cartilage, and attached by a thread to one of the levers of fig. 110. The magnification of the movement need not be greater than two-fold, and the loading should be effected isotonically and varied mutil the best excursion of the lever is obtained.

The first few records of figs. 160 and 161 show the form of the tracing. A rise of the lever is caused by a contraction of the diaphragm slip and therefore represents an inspiration, a fall, expiration. It is seen that relaxation of the diaphragm is carried out very rapidly, much more so than the corresponding contraction. The rate of breathing is rapid, at times as fast as two per second, at other times much slower. If the animal be deeply under the anæsthetic the rate is usually somewhat slowed. To show the influence of nervous stimuli upon the rate and depth of breathing, the following nerves should be stimulated :-

1. The superior laryngeal.-We may stimulate this nerve in two ways. We may imitate the normal method by introducing into the larynx a curved probe whose end has been wrapped in cotton wool. In this instance, and indeed for all these nerve stimulations, the rabbit must not be too deeply under the anæsthetic. The result of this stimulation is shown in I, fig. 160. In II, fig. 160, is seen the effect of weak tetanising shocks applied directly to the nerve. In both cases we see that there is slowing of the respirations, pauses occurring in expiration. By contrasting these with the well-known result of the presence of a foreign body in the larynx we see quite clearly that the results are very different. The effect of the anæsthetic has been to very greatly diminish the effect upon the respiratory centre, and in the case of the electrical excitation of the nerve there seems to be no doubt that the impulses thus originated are of very different character from those normally passing along the nerve after it is stimulated. 
The same remarks apply with equal force to the other stimulations we are about to examine.

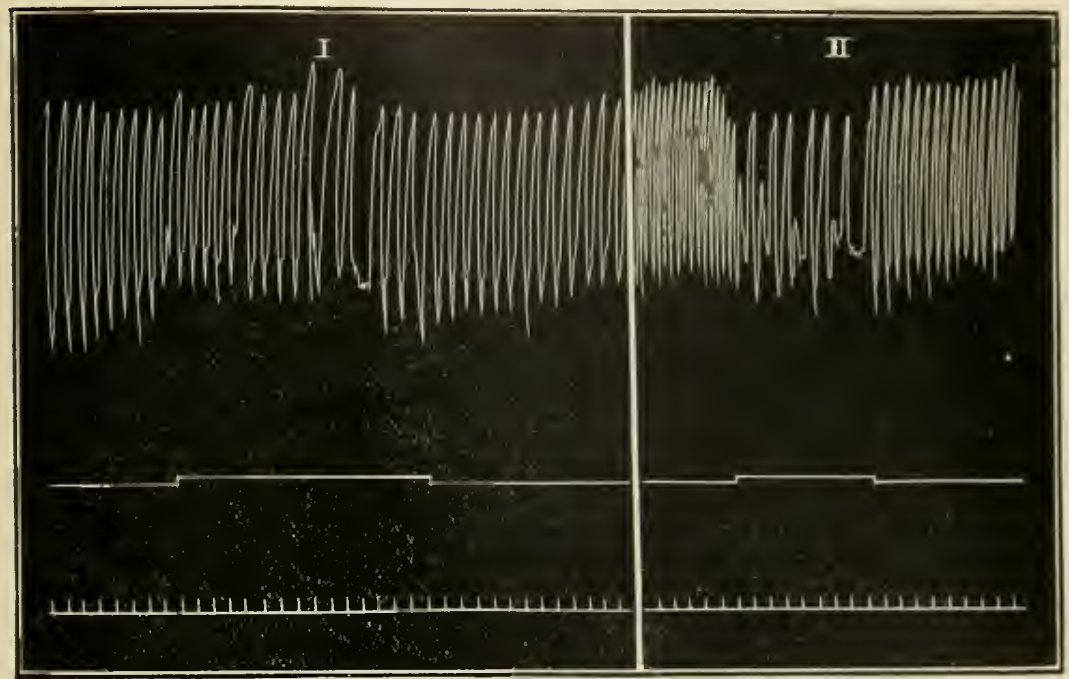

Fig. 160.-Alteration in Respiration on Stimulation of the Superior Laryxgeal Nerve: 1, By Mechanical Irritation of the Larixi; il, by Tetanisation of the Nerve. Rabbit.

2. The glosso-pharyngeal.-We can show the effect of this nerve on respiration, as in the previous case, by two modes of stimulation : (i.) By making the animal swallow a little water, and (ii.) by electrical excitation of the nerve. Fig. 161 gives the usual result of these stimulations. In I the animal was made to swallow about 2 c.c. of water placed in its pharynx. We see that inspiration is immediately inhibited, and that a very gradual relaxation occurred. During this time the animal was making rapid swallowing movements. In II with electrical stimulation we have a corresponding result. Breathing was at once inhibited for the time of about three respirations, and then recommenced, at first with shallow, then with decpening respirations. After stimulation ceased there are seen to be two alterations in the curve, namely at $a$ and $b$. These were synchronous with two swallowings. It is seen that in each case swallowing commenced in the middle of inspiration, which was then immediately inhibited, a slight expiration followed, and then as the swallowing ended the inspiration was completed.

3. Effect of section of the two vagi. - While the respiration is being recorded, the one vagus is lifted up by the loop previously placed round it and cut. After a short time the second is cut in a similar 
manner. The effect of section of the first vagus may be nil, no change either in rate or depth occurring; or there may be slight slowing

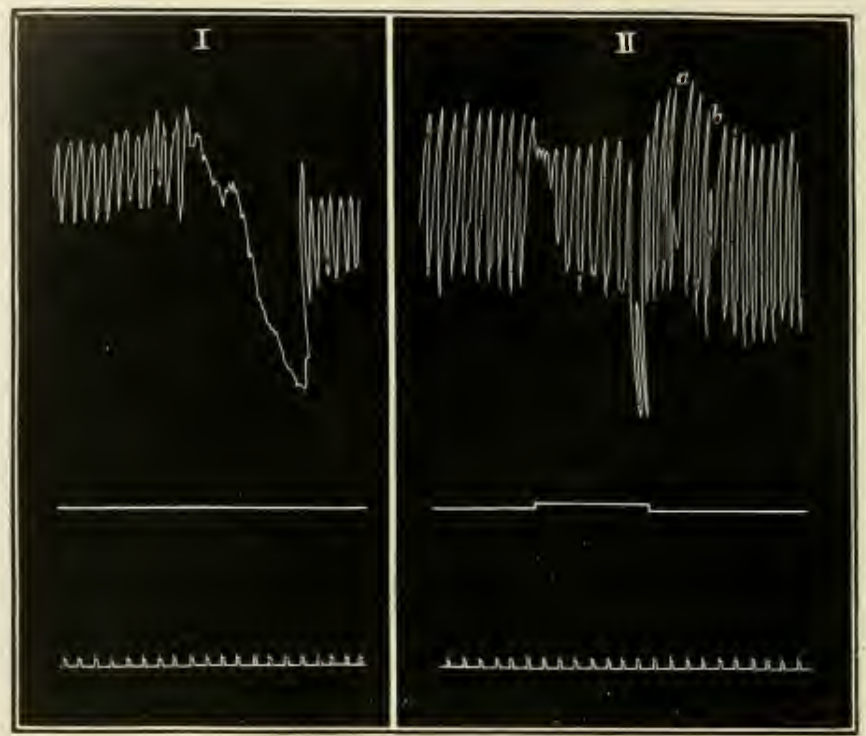

Fig. 161.-Effect upon Respiration of Stmulation of the Glosso-pharyngeal Nerve: i, by Making the Animal Swallow Water; if, by Tetanisation of THE Nerve.

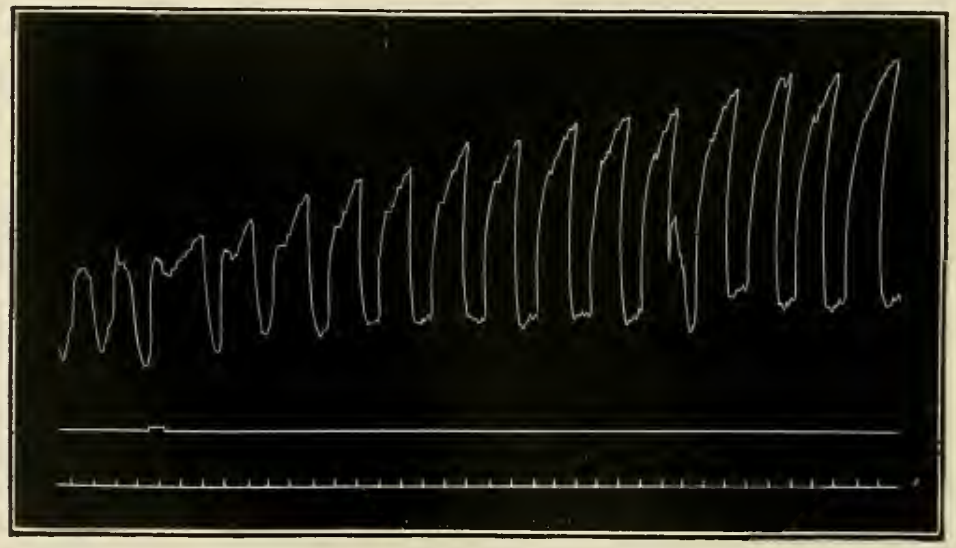

Fig. 162.-Result of Section of the Vagus, the other Nerve hatisg been Previously Divided.

accompanied by an increase in depth. The effect of section of the second vagus (fig. 162) is always to diminish the rate and increase 


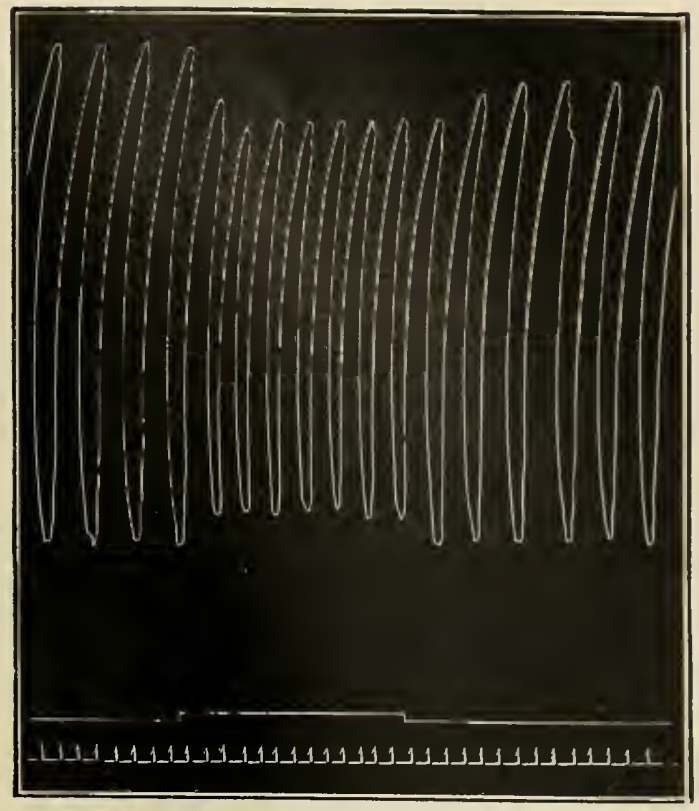

FIG. 163.

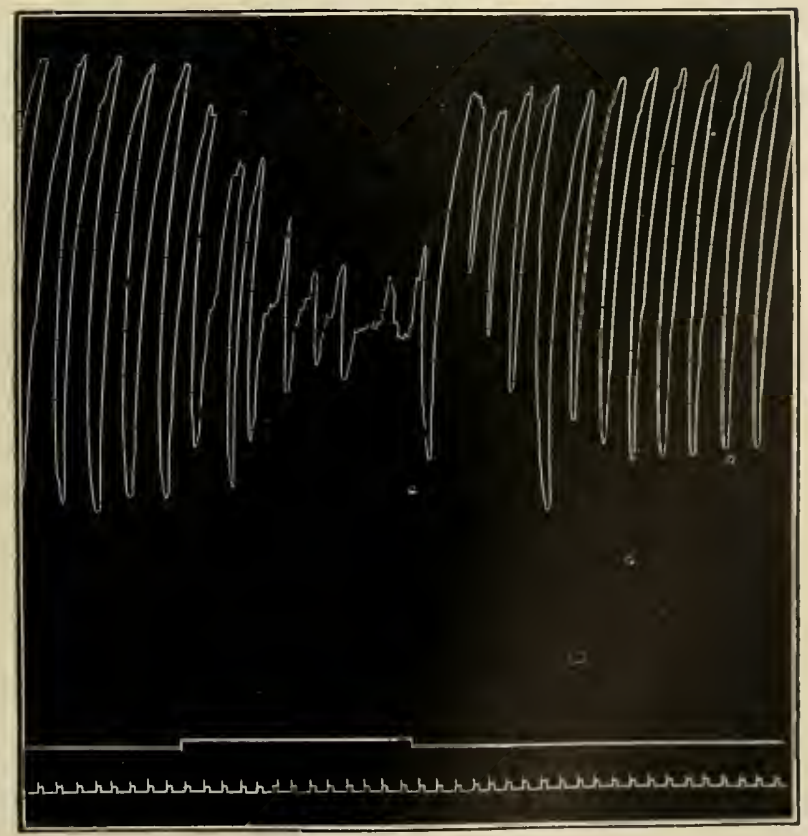

Fig. 164.

Figs. 163 and 164.--Stimulation of the Central Exd of the Vagus, botil Vagi having been Divided. In Fig. 163 tile Stresgti of Stimulus was Weaker thaN in Fig. 164. 
the depth of respiration, the increase in depth to a certain extent compensating for the diminution in rate.

4. Stimulation of the central end of the vagus.-What we may regard as the most typical result of stimulation of the central end, both vagi being cut, is that recorded in fig. 163 . In this case the tetanisation was very weak and caused an acceleration in rate from 32 respirations per min. to 36 per min., and the amplitude of the record diminished from $5 \mathrm{~cm}$. to $4 \mathrm{~cm}$. The diminution in extent is seen to be brought about by a less complete relaxation, as well as by a less extensive contraction. This result is much better obtained if the anæsthesia be not too deep. If the strength of stimulation be increased the effects are found to vary considerably. A common result is that reproduced in fig. 164, where it is seen that there is a gradually increasing tendency to standstill with the diaphragm neither relaxed nor contracted, but in a state of mid-contraction. In some cases standstill is produced in an inspiratory phase, inspiratory tetanus; in others, again, in an expiratory phase, expiratory tetanus.

\section{RECORD OF THE RESPIRATORY MOVEMENTS IN MAN}

Various instruments have been devised for recording the movements of the thorax in man. Of these the two following should be examined :-

Experiment 1.- Take a tracing with Marey's pneumograph (fig. 165). The instrument consists of a thin flat iron plate, $f$, with two stout bars of brass at either end. Attached to one bar is a tambour, $h$, which moves a lever, $b$. To

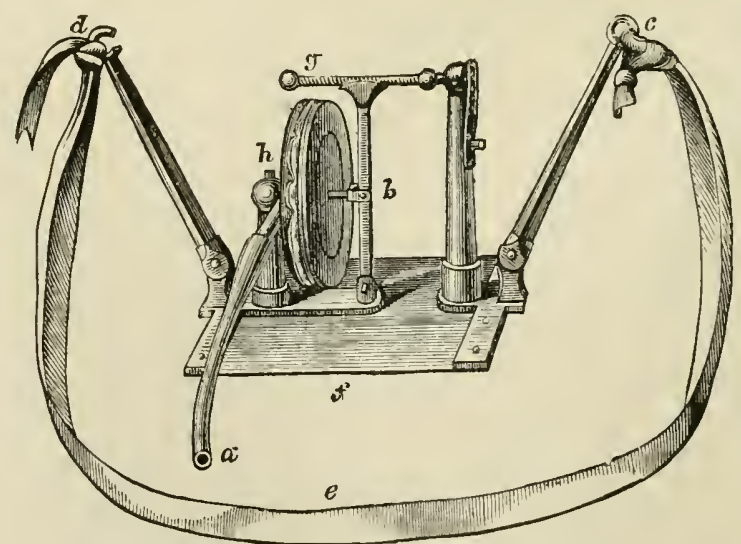

Fig. 165.-Marey's Pneumograph. (McKendrick.)

the other is fixed a vertical bar with a horizontal screw, $g$, which fits into the upper part of the lever $b$. By the band $c e d$ the apparatus is tied firmly on to the chest. Then with each inspiration the plate $f$ is bent, the rertical bar 
pulled from the tambour, which is therefore expanded. The variations in volume of the tambour are then recorded by a second tambour.

Experiment 2.-Take a tracing by Burdon-Sanderson's stethometer.

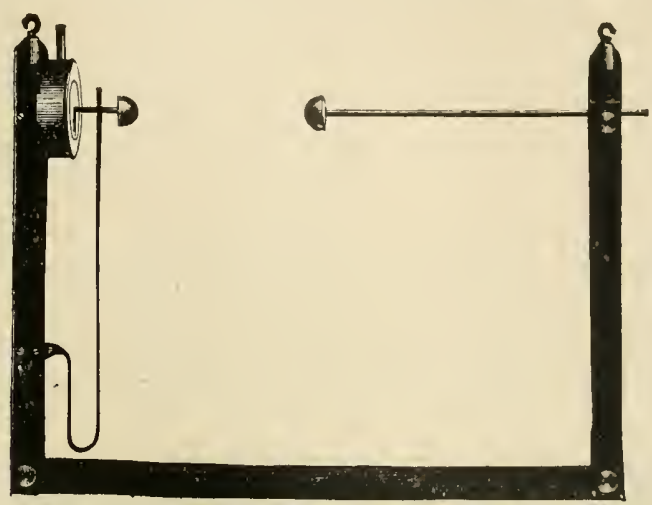

INST. CO. LTD. CAMb.

Frg. 166.-Sanderson's Sththoueter.

Fig. 166 shows the general form of the instrument, and fig. 167 the way in which it is fitted to the chest for recording the ehanges in transverse diameter. It consists of a large tambour A with a central dise of metal fixed to its

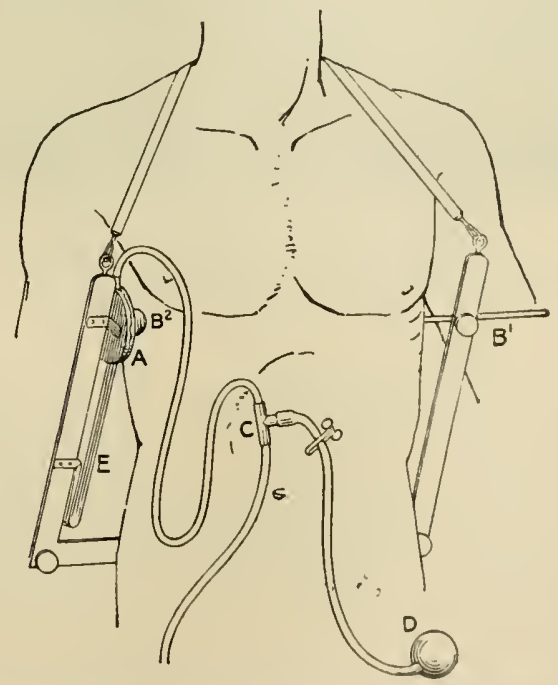

Fig. 167.-Mode of Applyng the Stethometen to Reconi Chasens in Transverse Diameter of thl Chest.

rubber membrane. An ivory knob, $\mathrm{B}^{2}$, attached to one end of a screw carried at the end of a spring $\mathrm{E}$ serves for aljustment to one side of the chest. At 
the other side is another knob on the end of a bar, $\mathbf{B}^{1}$, for adjustment to the corresponding point on the opposite side. An increase in diameter leads to a separation of the two knobs, only one of which can move relatively to the framework. This drives air out of the tambour and causes a rise of the lever of the recording tambour. The most important diameters to employ are (1) that which connects the 8 th rib in the axillary line with the same rib of the opposite side, and (2) that from the lower end of the sternum to the 8 th dorsal spine.

Record the changes in these two positions (1) during quiet breathing, (2) while sipping a glass of water, and (3) while swallowing a mouthful of biscuit.

The results obtained will be similar to those reproduced in fig. 168, which were taken with the instrument recording changes in the transverse diameter of the thorax. It is seen that inspiration and expiration each occupied about the same time; that at first the movement is rapid

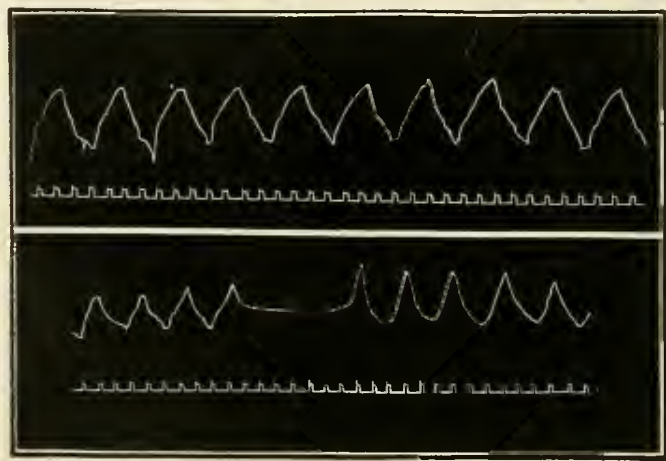

Fig. 168.-Record of Changes in the Transverse Diameter of the Thorax during Respiration (Man). The Upper Curve taken during Quiet Breathing: the Lower Curve shows the Effect of Swallowing. Time Tracing SECONDS.

in either direction, and then gradually slows. In the lower of the two tracings the inhibition of respiration during swallowing is recorded. A glass of water was slowly swallowed, and respiration was at once inhibited at the commencement of expiration, remaining in that state until swallowing ceased, when a fresh and rather deeper inspiration followed. From this time respiration resumed its usual characters. 


\section{CHAPTER XX}

\section{DEMONSTRATION OF THE SECRETION OF SALIVA FRON THE SUB- MAXILLARY GLAND OF THE DOG}

A DOG is secured to the animal holder and placed under ether; the hair is then clipped from the jaws and neck and the skin cleaned with a wet sponge. The necessary operation ${ }^{1}$ is then carried out in the following stages :-

1. Make an incision along the inner border of the lower jaw, beginning about its anterior third, a little in front of the insertion of the digastric muscle, and extend it backwards to the transverse process of the atlas, dividing the skin and platysma. 2. Expose the

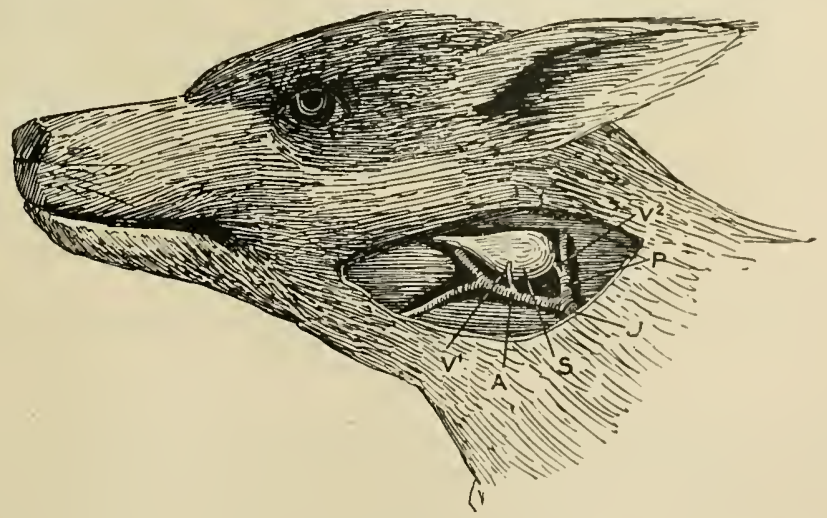

Fig. 169. - The Relation of the Veins to the Subuaxhlalix Glasi in tile Dog. (After Bernird.)

jugular vein (fig. 169) at or near the point where it divides into two branches, A and 1 , and lay bare those branches also. One of them, $P$, passes upwards behind the gland; the other, $A$, passes forwards below it and then divides into two branches. The gland itself has two veins: one of them, $\mathrm{v}^{1}$, comes from its lower side and enters

1 The description of the operation is taken from the Handbook for the Physiological Laboratory, by Brunton, Foster, Klein, and Sanderson. 
the vein $\mathrm{A}$. The other, $\mathrm{V}^{2}$, comes from its posterior aspect and enters the vein P. Sometimes one vein is the larger, sometimes the other. 3. Tie both branches of the lower division of the jugular a little beyond $\mathrm{v}^{1}$. Tie the upper of the two branches where it crosses the ramus of the jaw and remove the part between the ligatures. 4. Tie the other division (P) on the distal side of the place where it receives the gland vein, $\mathrm{v}^{2}$. 5. Remove the cellular tissue from the surface of the digastric, and from the groove between it and the masseter. Be careful not to injure the facial artery, and the duct of the gland which runs forwards and inwards between it and the masseter. 6. Separate the digastric muscle by means of a director or

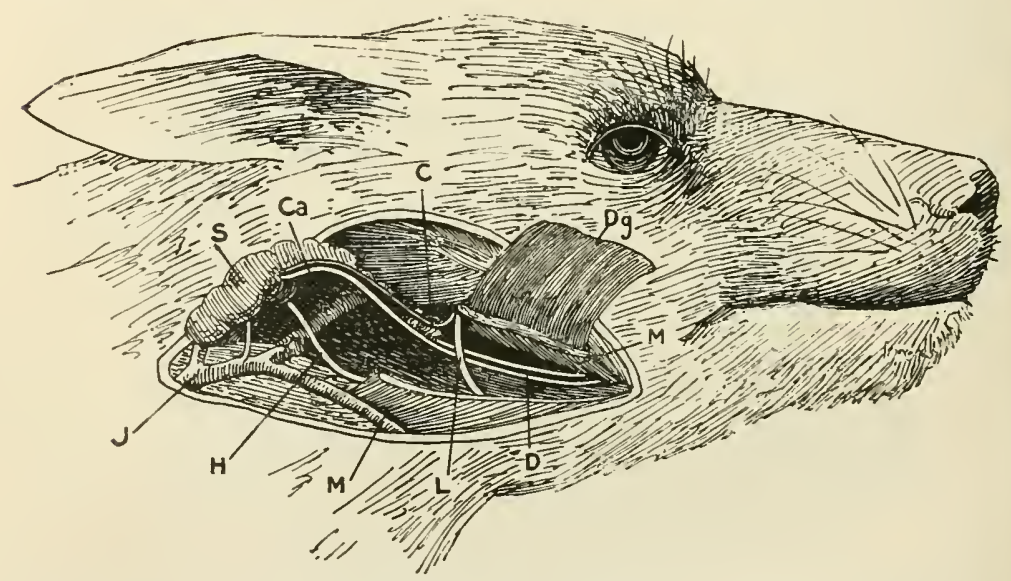

Fig. 170.-Relations of the Duct and Nerves of the Submaxillary Gland in the Dog. (After Bernard.)

aneurism needle from the facial artery. Tie the arterial twig which supplies the muscle. Separate the muscle from its attachment to the jaw, or divide it about its anterior third, cutting it through very carefully so as not to injure the duct and nerves which lie below it. 7. Lay hold of the lower end of the digastric with a pair of artery forceps and draw it backwards. This brings into view a triangular space whose apex is directed forwards, and whose base is formed by the reflected digastric. Its lower margin (the dog being supposed to be in the upright position as in the figures) is formed by the geniohyoid muscle, and its upper one by the ramus of the jaw and the lower edge of the masseter. The anterior half of its floor is formed by the mylo-hyoid muscle, on which some nerves ramify. The carotid artery enters the triangle at its lower angle and runs along 
its base, giving off first the lingual artery, secondly the facial. Just as the carotid begins to pass in front of the digastric, it is crossed by the hypoglossal nerve, $\mathrm{H}$, and is accompanied by filaments of the sympathetic. At the upper angle of the triangle several structures pass from it to the hilum of the gland, close to the margin of the digastric. These are: 1 , the duct; 2 , the nerves; 3 , the principal artery of the gland. The artery is given off by the facial. It lies beneath the nerves, but is easily reached by drawing them aside. 8. Carefully isolate the digastric by a director or aneurism needle from all the structures just mentioned. Divide it close to its insertion into the temporal bone. 9. Divide the mylo-hyoid muscle, $\mathrm{M}$, eutting its fibres across about their middle, and reflect the upper half, taking care not to injure the mylo-hyoid nerve which lies upon it, and tying all the veins which come into view on its surface with a double ligature. This brings into view the lingual nerve, $\mathrm{L}$, which issues from under the ramus of the jaw just opposite the groove between the digastric and masseter muscles, and, after passing across the floor of the triangle towards the middle line, enters the mucous membrane of the mouth. 10. Draw the parts a little towards the middle line with the fingers, and follow the lingual nerve to the ramus of the jaw. A small twig, c, will then be seen, which passes off from its posterior aspect, bends down, making a sort of loop, and then runs backwards to the gland in close relation to the duct. This nerve is the chorda tympani. In the angle between the chorda and the lingual lies the submaxillary ganglion. 11. Isolate the chorda and pass a thread under it, so that the nerve may be raised from its place at will. 12. To reach the sympathetic divide the hypoglossal nerve, $\mathrm{H}$, just where it crosses the carotid, and lift up its central end. Close to the inside of the carotid lies the vagus, and when this is raised the sympathetic is seen lying underneath and to the inner side of it. The sympathetic separates from the vagus at this point, and passes to the superior cervical ganglion. From the ganglion, fibres accompany the carotid and enter the gland along with its arteries. The ganglion can easily be found by following the carotid filaments backwards. 13. Place a cannula in the submaxillary duct. The ducts of the submaxillary and sublingual pass along the middle of the triangle close to one another. The submaxillary duct lies closer to the ramus of the jaw, and is larger than the sublingual duct. Isolate it slightly with an aneurism needle. Pass under it a thread for the purpose of tying in the cannula. Pass under the duct a smooth splinter of wood or a piece of card, half an inch long by one-eighth of an inch wide, on which it may rest. Close the duct as near the mouth as possible with a clip, or tie a thread round it, so as to obstruet it. Raise the chorda by the threald 
which has been passed round it, and stimulate it by a weak interrupted current-the purpose of this is to distend the duct with secretion, and thus render the introduction of the cannula much easier. Open the duct with a pair of sharp scissors, insert the cannula, and tie it in.

To show variations in rate of secretion the secretion may be collected in small glass tubes or capsules, having attached a short piece of rubber tubing to the end of the cannula. The following plan is a very good one for class demonstration. A long piece of glass tubing with thick walls and of about $1 \mathrm{~mm}$, bore is taken. To one end of this a piece of rubber tubing is fixed, in the course of which is inserted a glass T-piece, the lateral orifice being closed by a piece of tubing and spring clip. The rubber tubing and a few inches of the glass tubing are filled with a solution of methylene blue. The cannula is then filled with fluid and connected to the long glass tube by the rubber tubing. The glass tubing is then held in a horizontal position against a white surface, so that the coloured column of fluid stands out clearly. It is also convenient to have the tule marked with transverse lines at short intervals. When the demonstration is being made to large classes, it is often convenient to project an image of the coloured column on to a screen by means of a lantern. As saliva is secreted the coloured fluid is moved along the horizontal tube, and its rate can be very accurately watched. The meniscus of the fluid can at once be brought to any position of the tube by aid of the T-piece on the rubber tubing. By opening this some fluid may be forced out or sucked in, if the end of the rubber tubing be immersed in fluid.

We may now show the chief facts in the rate of secretion of saliva by the following experiments :-

1. Observe the rate of flow of saliva from the unstimulated gland. In animals anæsthetised with ether this rate is, as a rule, greater than when other anæsthetics are employed.

2. Stimulate the chorda.-Lift up the nerve, place it upon a pair of shielded electrodes, and tetanise it with weak induced currents. The rate of flow of saliva is very greatly accelerated.

3. Stimulate the sympathetic.-The rate of flow, in the case of the dog, is no greater than before stimulation.

4. Observe the vaseular changes in the gland on stimulation of the two nerves. When the chorda is stimulated the gland will be distinctly observed to become redder, due to dilatation of its blood vessels. On the other hand, sympathetic stimulation is followed by a paling of the gland, due to constriction of its vessels.

5. Take a camel's-hair pencil moistened with a $0 \cdot 1$ per cent. solution of nicotine in 1 per cent. $\mathrm{NaCl}$ solution and paint the submaxillary ganglion with the solution of nicotine, taking care to limit the action of the nicotine to the submaxillary ganglion. Now stimulate the chorda once more and a rapid flow of secretion is again obtained. In the next place paint the chorda with the solution of nicotine at a spot about $1 \frac{1}{2}$ inch from the hilum. Then once more stimulate the chorda near its origin from the lingual. A free flow of saliva is 
still obtained. Lastly, paint the chorda with the nicotine solution, just at its entrance into the hilum, and again stimulate. No secretion is obtained.

Finally apply the electrodes to the hilum of the gland, when on stimulation a free secretion will again be obtained. This experiment with nicotine demonstrates the existence of nerve cells on the course of the chorda fibres. Nicotine in minute doses is known to paralyse nerve cells without injuring the nerve fibres, though in large doses it paralyses nerve fibresas well. The experiment therefore prores that the nerve cells in the submaxillary ganglion are not placed on the course of chorda fibres running to the submaxillary gland. They are, in fact, known to lie on the course of secretory fibres running to the sublingual gland. The result of painting the chorda with nicotine proves that the nicotine solution is not strong enough to paralyse the nerve fibres. The absence of secretion on stimulation after the hilum has been painted with nicotine proves that there are gland cells on the course of the secretory fibres which are placed on the nerve at or near its entrance into the hilum. This is further confirmed by obtaining a secretion on subsequent stimulation at the hilum, for then the post-ganglionic fibres are excited. 
Experiment 1.-Destroy the brain of a frog, leaving the spinal cord intact. This should be done with a blunt instrument, so that the animal loses as little blood as possible. If the animal be tested immediately by pinching one of the toes it may or may not respond by a movement of the leg. If it be kept for half an hour or a little longer it will very readily respond. The absence of response just after the destruction of the brain is due to the shock of the operation, but this rapidly passes off in the case of the frog.

After this period of rest note the condition of the frog. It lies on the table without any attempt at spontaneous movement. Its head and body lie in contact with the table, whilst in an intact frog they are always inclined to the surface on which it rests. The legs are usually drawn up and the fore limbs may be extended at right angles to the axis of the body or may lie folded over the sternum. The eyes are closed and no respiratory movements are attempted. The general attitude of the animal should be contrasted by comparison with that of an intact frog.

Having determined these points its behaviour when its position is altered should be investigated. If a leg be stretched out it is usually drawn up again to its original position as soon as the fingers are withdrawn. Place it on its back; it will lie at rest practically in the position in which it is placed. It makes no attempt to turn over into its previous position, whereas an intact frog immediately turns over as soon as it is allowed to. Suspend the frog by passing a bent pin through the lower jaw. The pin does not act as a stimulus, and so cause reflex movements, because the centres of the sensory nerves of this part have been destroyed in pithing the brain. The frog may thus be supported in any convenient manner and the reflex movements in response to various forms of stimuli studied.

1. Mechanical.

(a) Pinch any one of the toes of the right foot; the right leg is drawn up. If the toe be held you will feel a puil on the fingers, tending to lift the leg. This pull is not continuous, but varies in strength. Pinch a toe of the left foot; the left leg is drawn up. Pinch the skin of the 
flank; the leg of the same side is rapidly drawn up, as if to push away the object stimulating the skin. These are all instances of unilateral reflex movements, and may be extended in many directions.

(b) Pinch the skin over the anterior surface of the pubes or round the anus; both legs are now drawn up to rub the spot stimulated.

(c) Pinch one of the toes gently. With a mild stimulus there may only be a slight flexion at ankle and knee. Increase the strength of the pinch, and the movements of the leg become more marked. If the strength be still further increased, movements of the opposite leg will also be produced.

2. Electrical.

(a) Single induction shochs.-Apply a pair of wire electrodes from the secondary coil to the skin of the leg. Stimulate with single induced shocks, gradually increasing the strength of the shock. No reflexes are produced, though a twitch produced by the direct excitation of the muscles may be produced if the stimulus be sufficiently strong.

(b) Repeated induced shocks.-At first employ very weak stimuli. A reflex is quite readily obtained. This forms an instance of summation of effect. A single stimulus produces no result; but if repeated, even though weak, the effects are gradually summed up until they are able to produce a series of reflex impulses.

\section{Chemical.}

(a) Take some 0.2 per cent. sulphuric acid in a small beaker, and with the frog suspended move one leg to one side with a loop of thread or a glass rod, and then immerse the other foot in the dilute acid. In a short time the leg is withdrawn from the acid, but will again relax, dip into the acid and be withdrawn for a second time. Remove the acid and wash the skin thoroughly with water to remove all traces of acid. Allow the frog to rest for a few minutes.

In all cases where a chemical irritant has been employed it is of the greatest importance that this resting period should be sufficiently long - five to ten minutes-before a fresh excitation is attempted, otherwise the results obtained are not characteristic.

(b) Repeat the experiment, using 10 per cent. acetic acid instead of the sulphuric.

\section{Thermal.}

Touch one of the toes with a heated wire; the foot is withdrawn.

Having shown by these experiments that a reflex act is produced in response to mechanical, electrical, chemical, or thermal stimuli, proceed next to study the characters of the reflex. 


\section{The latent period or reflex time.}

Arrange a time marker recording $\frac{1}{4}$ seconds and a signal to write vertically over one another on a blackened surface set to rotate at about $1 \mathrm{~cm}$. per $2 \frac{1}{2}$ seconds. Take some of each of the four strengths of sulphuric acid, 0.1 per cent., 0.2 per cent., 0.3 per' cent., and 0.4 per cent., in small beakers, labelling each. Dip the foot up to the ankle in the weakest solution, recording the instant of immersion by closing the key of the signal. Wait until the foot is withdrawn and then open the key of the signal. Count the number of oscillations of the time marker which have occurred during the closure of the current through the signal. This is the reflex time for that strength of acid. Wash the skin thoronghly, allow the frog to rest for a time, and then repeat the experiment, using the 0.2 per cent. solution. In the same way repeat for the other strengths of solution.

Arrange the results thus obtained in tabular form as in the following instance:-

\begin{tabular}{c|c} 
Strength of Acid & Reflex Time in Seconrls. \\
\hline Per cent. & \\
$0 \cdot 1$ & $3 \cdot 25$ \\
$0 \cdot 2$ & $3 \cdot 0$ \\
$0 \cdot 3$ & $2 \cdot 25$ \\
$0 \cdot 4$ & $2 \cdot 0$
\end{tabular}

2. Purposive character of the reflex.-In all the reflex actions studied it will be noticed that the muscular response is a very complex one. It is in no way an irregular series of twitches of the limb muscles, but is a movement similar in nature to those carried out by the frog during its life. It involves several muscles, each of which contracts at the right instant, to the proper extent, and at the proper rate, and another set of muscles which relax to the right degree, and at the right time; i.e. it is a co-ordinated movement. In addition to this the response obtained is different according to the part stimulated, and when examined is seen to tend either to remove the irritating body, to move that part of the body from the irritant, or to remove the whole body. This purposive character of the response is well seen in the following experiment :-

Take some squares of filter paper, about $4 \mathrm{~mm}$. each side, and dip them into some 20 or 40 per cent. acetic acid. Remove the excess of acid from one of these and place it upon the flank of the frog. After a latent period the limb on the same side is drawn up, and the flank rubbed with it as if to wipe away the irritating body. In this the movement frequently succeeds. Wash the skin, and after a period of rest apply another square and hold the leg of the same side. The leg of the opposite side will probably be moved so as to remove the irritant. Wash the skin again and study the effects of altering the position of the irritant. In all cases characteristic but different movements are produced.

3. Irradiation of reflex movements.-In many of the experiments we have so far tried it has been noted that with a given stimulus 
applied, say, to one part of a limb, a fixed response is obtained; but if the strength of the stimulus be increased, the movement may involve parts on the other side. This is termed irradiation. In studying the question of response with regard to strength of stimulus employed it is found that as the strength increases the stimulus tends to spread first to the same level on the opposite side, e.f. from one leg to the other ; and that only when the stimulus is still further increased does it tend to spread upwards and downwards to fresh levels. There are conditions in which the extension of a stimulus to other parts is greatly facilitated; as, for instance, in strychnine poisoning.

Take a frog with its brain destroyed and inject 2 drops of a 0.5 per cent. solution of strychnine sulphate. In a few minutes stimulation of the skin in any part of the body excites a general convulsion of the whole body. All the muscles are thrown into violent tetanic spasms and the limbs become extended and rigid. The tetanic spasm passes off to be at once repeated on even the slightest stimulation, such as a tap on the table. Note that these contractions are not co-ordinated muscular inovements, but are general tetanic contractions.

Destroy the cord by pithing. At once the contractions cease, showing that the effect of strychnine is one acting directly upon the cord, not upon the nerves and museles.

4. Inhibition of reflexes.-Employing the frog at first taken, expose the upper end of the cord and place a crystal of sodium chloride upon it. After a minute try to obtain any of the reflexes previously obtained easily. They will now be found not to occur, and may not be produced even though the strength of the stimulus be considerably increased. 


\section{CHAPTER XXII}

SOME EXPERIMENTS IN THE PHYSIOLOGY OF THE EYE.

ACCOMMODATION, OPHTHALMOSCOPY, COLOUR SENSE, PERIMETRY

Accommodation.--An object can be seen distinctly if it be placed close in front of the eye, or if it be removed to some distance from the eye. But as an object can only be seen distinctly if its image be accurately focussed on the retina, it follows that the eye possesses some mechanism by means of which any image can at will be focussed upon the retina. This means consists in a power of alteration of the curvature of the lens. That this is the optical change produced may be proved by studying the images reflected from the curved refracting surfaces of the eye, while the eye is fixed first upon a near and secondly on a distant object. The power of throwing a distinct image, now of a near object and now of a distant one, upon the retina is termed accommodation.

Before examining these images reflected from the eye make out the following points upon a series of watch glasses of different curvatures :-

1. When light falls upon the surface of a medium of different refrangibility from that in which it is travelling some of the light is reflected, even though the medinm be transparent.

2. Hold a lighted mateh in front of a sheet of polished glass. An erect image of the flame is observed of the same size as the match.

3. Repeat, but use the convex surfaces of watch glasses of different degrees of curvature, starting with nearly flat ones and choosing a series in which the amount of curvature gradually increases. In all these cases images are produced which lie behind the glass, i.e. they are virtual, and which decrease in size as the curvature increases. Note further that the images are erect, and that, as they become smaller with the increased curvature of the surface, the rirtual image comes to lie nearer to the reflecting surface.

4. Next examine the images produced from the concave surfaces of the series of watch glasses. These are all inverted and lie in front of the surface, i.e. they are real. They are all smaller than the object, and diminish in size and move towards the surface as the latter becomes more curred.

Now examine the images of a candle formed from reflection from the eye. Hold the candle a little to one side and in front of the observed eye and then look obliquely at the eye. One image is very clearly seen : it is erect, small and virtual, and therefore comes from a convex surface. The positions at which reflected images can be 
formed, i.e. the surfaces separating media of different refrangibility, are (1) the anterior surface of the cornea, (2) the anterior surface of the lens, and (3) the posterior surface of the lens. We shall find that reflected images are formed by these three surfaces and that the first produces the brightest image because it separates two media possessing a greater difference of refrangibility than is the case with the other surfaces. The clear image already described comes, then, from the anterior surface of the cornea. On examining carefully in a darkened room, a second image will be observed lying apparently behind the first, much less bright than that image, but erect and somewhat larger than the first image. This image is therefore produced by a convex surface lying behind, and less curved than the anterior surface of the cornea. It is from the anterior surface of the lens. On further examination a third image can be made out less bright than either of the preceding; it is, moreorer, inverted, real, and smaller than either. It comes, therefore, from a concave surface more curved than either of the preceding. This surface is the posterior surface of the lens. These three images are called the Sanson-Purkinje images. The changes in size and position of these images may be utilised to prove that the curvature of the lens alters during accommodation. For this purpose the observed eye should first be accommodated for a distant object and then for a near one, the reflected images being observed as the change occurs. It will be found that the first image remains unchanged, but that the second inage becomes smaller and moves nearer to the first. This proves that the surface forming it, the anterior surface of the lens, becomes more convex. The movement towards the first image is due to two causes: firstly, that the anterior surface of the lens becomes more conrex; and secondly, that the anterior surface of the lens approaches a little nearer to the cornea.

The third image is also found to change, but to a much less degree. It becomes a little larger, and appears to move further from the second. The apparent size and position of this image are, however, modified by the fact that it is viewed through the two surfaces of the cornea and lens; and careful measurements of this image hare shown that the changes observed are due, not to a change of curvature of the posterior surface of the lens, but to the change in the anterior surface through which it is viewed.

The observation and mneasurement of these images are much tacilitated by the phakoscope, an instrument devised by von Helmholtz for that pur. pose. It consists (fig. 171) of a triangular box whose angles are cut off. At one angle two prisms, $b$ and $b^{\prime}$, are fixed which, when illmminated, concentrate two beams of light upon the observed eye. At the opposite angle, $a$, is an aperture 
for the observer's eye, and at the third another aperture throngh which tho observerl eye looks straight forward through an aperture above $c$. 'The observed first looks at a far object and the images are then noted, especially with re-

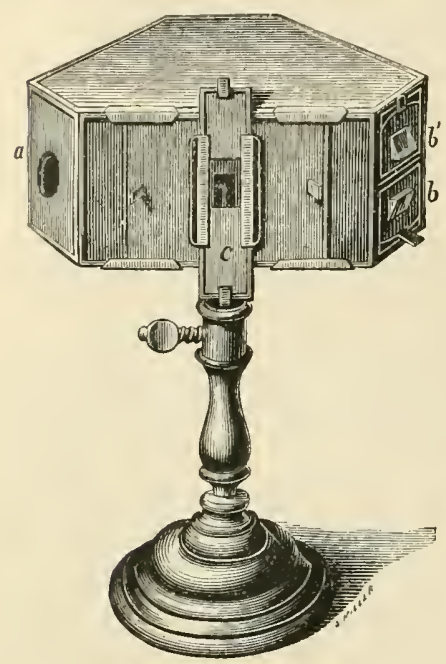
gard to their size and position. The images obtained are given in fig. $172 \mathrm{~A}$. The two bright ones to the left are from the ante. rior surface of the cornea, the lower one being formed from $b$, the upper from $b^{\prime}$. The middle pair are much larger than the first, and the third pair are smaller than any, and inverted. Prove this by blocking $b^{\prime}$ with a card. The lower image of the right-hand pair and the upper images of the other two pairs lisappear

The observed eye is now accommodatel for a near object by looking at a pin in the shutter $c$, when the images

Fif. 171.-The Phakoscol'e. (McKeNDRICK.)
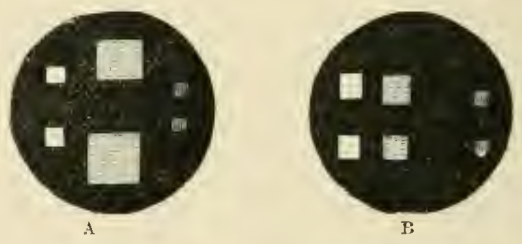

Fig, 172.-The Reflected Images as Seen in the Phakoscope: a, while the Eve is at Rest ; B, DURing Accomadodion. (McKeNDrick.)

change to those seen in E, tig. 172. The wirldle pair become smaller, lie closer to one another, and approach the first pair. 'The third pair separate a little from one another and become a little larger.

The changes in the media of the eye, brought about during accommodation, are further illustrated by the following experiment, known as

Scheiner's Experiment.-Take a long strip of wood, and to one end fix a card rertically, and pierce this with two fine pin-holes lying close together and on a horizontal line. They must be se near each other that they both lie within the diameter of the pupil. Fix two needles vertically on the wood, one about eight inches in front of the card, the other about twenty-four inches away. Close one eye and look through the pin-holes in the card at the two needles.

1. Fix the eye upon the distant needle. A clear single image is obtained of this, bnt two blurred images of the nearer needle are at the same time observed. Now close the right-hand pin-hole, when the left-hand image of the near needle disappears. On closing the left-hand pin-hole the right-hand inlage disappears.

2. In the second place look at the nearer needle, when a double image of the far needle will be observed. Now close the right-hand pin-hole, when the right-hand image disappears. On closing the left-hand pin-hole, the left-liand image disappears.

The meaning of this experiment will become clear from a study of 
fig. 173. I illustrates the condition of things in the first part of the experiment. $\quad \mathrm{C} \mathrm{C}^{1}$ is the card, $\mathrm{A}$ and $\mathrm{B}$ the two pin-holes; $\mathrm{r}$ is the far needle in transverse section, and $\mathrm{R}$ the near needle. The eye is accommodated for the far needle $\mathrm{P}$, and the rays of light from it (e.g. the continuous lines of the figure) which pass through $A$ and $B$ meet on the retina at $p$. Hence there is a clear single image of $\mathbf{P}$. The light from $\mathrm{n}$ (i.e. the interrupted lines of the figure) are not, with the position of the refracting surfaces, sufficiently refracted to meet on the retina, and consequently fall upon it in two patches at $r^{1}$ and $r^{2}$. These are able, however, to give a moderately good image, because they are only formed from the rays passing through two minute
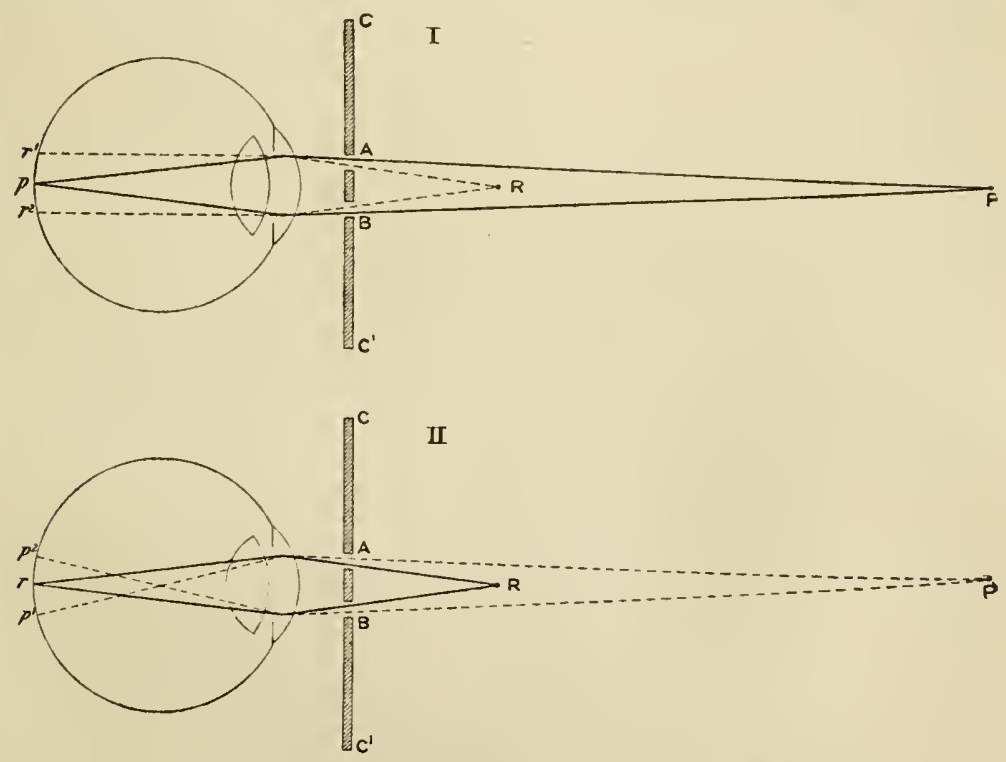

Fig. 173. - To Ihlestrate Schenver's Experimext.

orifices, $\mathrm{A}$ and $\mathrm{B}$. On now closing the left-hand pin-hole, $\mathrm{A}$, the image at $r^{1}$ disappears. This lies on the retina to the left of the second image at $r^{2}$. But the left half of the retina is normally concerned with objects lying to the right, and vice versa. Consequently the mind projects the image at $r^{1}$, as if it were coming from an object to the right, and the image at $r^{2}$ as from an object to the left. Blocking A therefore causes the right-hand image to disappear, and blocking 1 ; the left-hand image.

II gives the condition of things for the second half of the experiment. The eye is accommodated for $\mathrm{k}$, and the rays are more 
refracted than in the previous case. The rays from $\mathrm{k}$ meet accurately upon the retina at $r$, and produce a clear image there. The rays from $P$ are more refracted, and meet at a point in front of the retina, cross, and impinge at two positions, $p^{1}$ and $p^{2}$, upon the retina, where they give rise to the sensation of two images of the needle $P$ inclistinct and blurred. The image $p^{2}$ will be referred as coming from an object lying to the right of that caused by $p^{1}$. On now blocking the lefthand pin-hole $\mathrm{A}$ the image formed by $p^{1}$ disappears. This is the left-hand image. Similarly on blocking the right-hand hole $\mathrm{B}$ the right-hand image due to $p^{2}$ disappears.

The ophthalmoscope.-On looking at an eye the pupil always appears black. This is because most of the rays entering the eye are absorbed by the retinal and choroidal pigment, and those few which are reflected travel back along nearly the same path as that they took on entering the eye. To see any part of the interior of the eye, these reflected rays must enter the observing eye ; but as soon as the eye is placed to intercept them, it also blocks the course of the entering light. The first condition, then, is to be able to receive the reflected rays without at the same time intercepting the rays from the source of light.

This is attained by the instrument invented by von Helmholtz, the ophthalmoscope. The principle of this is to reflect light into the eye by a mirror in the centre of which is a small aperture through which the observer looks, and is thus able to receive some of the rays reflected from the interior of the observed eye.

There are two methods of employing the ophthalmoscope, which are known as the indirect and direct methods respectively.

I. The indirect method.-The person whose eye is to be examined is seated in a darkened room with a large steady flame placed a few inches from his head on his left side on a level with his eyes. For examining his right eye take the ophthalmoseope mirror in your right hand, and with the mirror towards the observed cye look throngh the central aperture, with the right eye so that (with the left eye closed) you can see the observed eye clearly. Now open the left eye, and watching the position of the reflected light rotate the mirror until the reflected light is thrown on to the eye. Now tell him to look steadily at some object behind you at the other end of the room. The pupil will now become a bright red. Get him to move his eye in varions directions and in one position, when it is turned a little inwards; the red will change to a yellowish colour. This indicates the position of the optic disc. Now talke the large biconvex lens in the left hand, holding it vertically about 2 to 3 inches from the eye, steadying your hand by resting your little finger on the temple. Your eye should be about 15 inches from his. In this position an image of the funclus of the eye will be formed by the lens about 2 or 3 inches in front of it, i.e. about 10 inches from your eye, and you will be able to see this image, which if the observed eve have not been noved will be one of the optic disc. Most beginners find some difficulty in aroiding the reflection from the cornea and in adjusting the accommodation, and the distance of the head, so as to see the image clearly. The head must be 
slowly moved a little nearer to or further from the observed eye, and at the same time an attempt made to accommodate the eyes for a point between the observer and the lens.

Fig. 174 gives diagrammatically the course of the light in this instance. $\mathrm{E}$ is the observed eye and $\mathrm{u}$ ir the concave mirror with its central aperture,

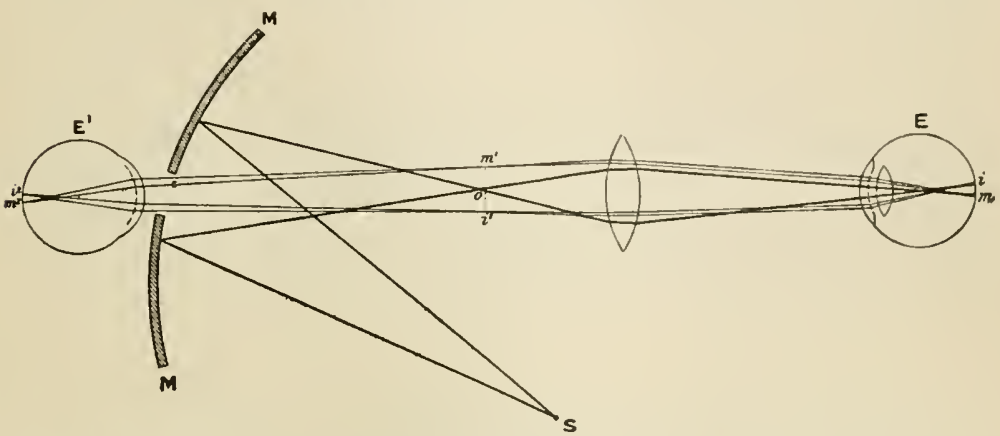

Fig. 174. - The Course of the Light in the Indrect MIethod of Euploina The Ophthalioscope.

$\mathrm{s}$ a source of light the rays from which falling upon the mirror are reflected to form an image at 0 . They then diverge, but are again condensed by the lens, and entering the eye, $\mathrm{E}$, form a second image just behind the lens; they then again diverge and diffusely illuminate the fundus oculi. The rays of light reflected from two points, $i$ and $m$, on the fundus, diverging from the eye, are refracted by the lens to form an inverted real image, $i^{1} m^{1}$, larger than the object, $i m$. These latter rays then diverging are collected and focussed by the observing eye $\mathrm{E}^{1}$ to form an image $i^{2} m^{2}$ on the retina.

The indirect method of examination is most generally useful because it gives a large field of view under a low magnifying power (about five diameters). In the view obtained it must be remembered that all the parts are inverted, that seen to the right being from the left part of the fundus, and vice versa.

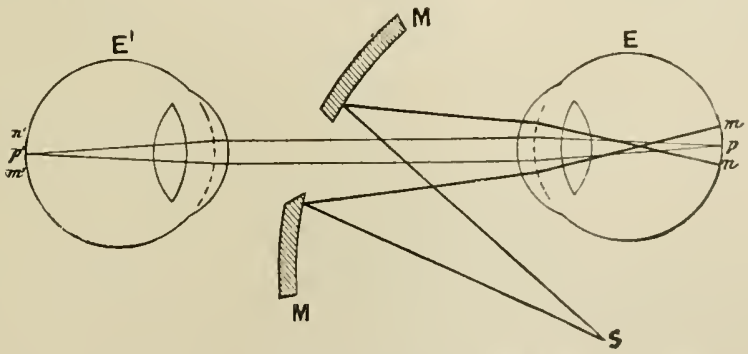

Fig. 175.-The Course of the Light in Eximising the Ere 13 the Direct Meth(]).

I1. The divect method.-In this method the eximination is made with the mirror alone, without the intervention of the biconvex lens. In this method a small concave mirror is used, and is brought as close as possible to the observed eye, which should be accommodated for distant vision. The 
reflected rays from any point of the fundus in a normal eye then emerge parallel, and the observer's eye should also be accommodated for distant vision in order that a clear image may be thrown npon his retina. As this latter is at first difficult and requires some practice, it will be found easier to insert a biconcave lens behind the aperture of the mirrol to render the rays divergent, and then the observer accommodates mntil distinct vision is obtained.

Fig. 175 shows the course of the rays of light when employing this metlod. $\mathrm{s}$ is the source of light and $\mathrm{n}$ is the mirror which reflects the rays of light, these are focussed by the eye being examined (E) to a point in the ritreous, and from this canse a diffuse lighting of the fundus. Rays of light issuing from a point $p$ emerge from the eye parallel to one another, and entering the observing eye $\mathrm{E}^{1}$ are brought to a focus. $p^{1}$, which lies on the retina, as the eye is accommodated for distant vision. Similarly a point $m$. will give rise to an image at $m^{1}$, and a point $n$ at $n^{1}$.

\section{HOLMGREN'S METHOD OF TESTING COLOUR VISION}

In this method a large number of sample skeins of worsteds of different colour's and shades of colour are employed. The colours include reds, oranges, yellows, yellowish greens, pure greens, blue greens, blues, violets, purples, pinks, browns, and greys. The method of testing consists in picking out one of the skeins and requesting the subject to be tested to select skeins from the pile which resemble it in colour. No two skeins are alike, so that the examinee is to pick out skeins which appear of the same colour, though they may be lighter, or darker, or of nearly the same shade.

When testing for colour blindness the following plan is recommended. A pale green, a purple, and a red skein are chosen and are termed test skieins.

I. The green skein is first picked out. This skein should be the palest shade of pure green, which is neither a yellow green nor a blue green. The examination is continued until the examinee has picked out all the other skeins of the same colour, or else placed with them one or more skeins of what are termed the confusion colours. These confusion colours which a colour-blind person will thus pick out are of various tints, according to the amount of his defect. Thus there may be greys, light reds, or light purples. The fact that any confusion colour is picked out is sufficient to show that he is colour blind. To determine the kind and degree of colour blindness the next test skein is given.

II. This is a purple skein, and should be midway in colour between the lightest and darkest purple in the heap. The test is continued until the examinee has picked out all the purples, or until certain confusion colours have been selected. 
A person who has been proved colour blind by the first test, but who only selects purples in the second, is incompletely colour blind.

If he select with the purples blue and violet, he is completely red blind.

If he select with purple only green and grey, he is completely green blind.

As a final and confirmatory test, the third test skein is presented.

III. This is a bright red skein of medium shade tending rather to a yellowish red.

The red-blind person chooses with the reds, greens and browns of darker shade than the skein presented.

The green blind chooses with the reds, greens and browns of lighter shade than that of the test skein.

\section{PERIMETRY}

When we wish to carefully examine any object we turn our eyes to such a position that the image of the object falls upon the forea centralis. This is termed direct vision. The vision produced by images formed on the peripheral parts of the retina is in contradistinction termed indirect vision. Indirect vision is much less acute than direct, but still the periphery of the retina is capable of appreciating movements or changes in intensity of light falling upon it.

In order to test the limits of indirect vision the perimeter (fig. 176) is employed. This consists of a vertical pillar carrying a horizontal axis which bears on one side a circular arc and on the other a vertical disc to which a chart can be fixed. A little holder can be moved along the are into any position. A second support terminating in a knob is provided, against which the cheek is held about an inch below the centre of the eye to be tested. The opposite eye is then closed, and the other one fixed in position by looking steadily at a white knob in the centre of the axis of the instrument. A chart is next fixed in posi-

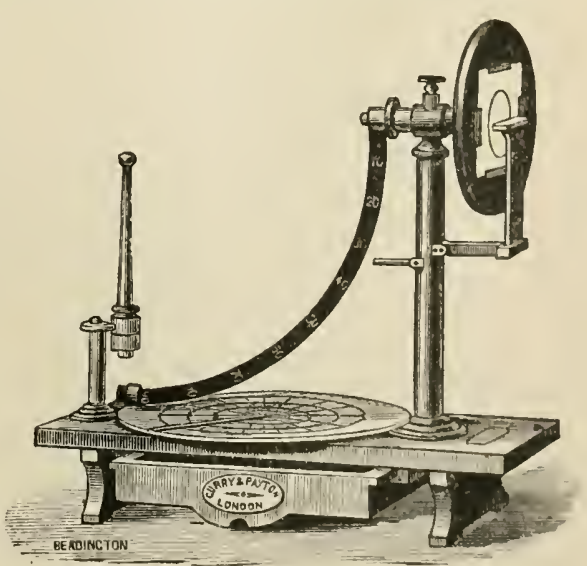

Fig. 176.-Priestlex-Sintis's Perimeter. (Halliburton.) tion in the vertical disc, and first a white square held in the earricr on the rotating arc. This is moved from the periphery towards tho centre until it is clearly observed, and its position then malied upon the chart. The arc 
is then moved into a fresh meridian and a fresh observation taken, and so on until a series of points have been mapped ont upon the chart which lie on the limit of distinct rision. These are united by a curved line, such as the dotted line in fig. 177, which represents the normal average field of vision for white light for the right eye.

Having determined this for a white object, next determine similar lines for coloured objects.

For the white object it is seen that the field of vision extends more

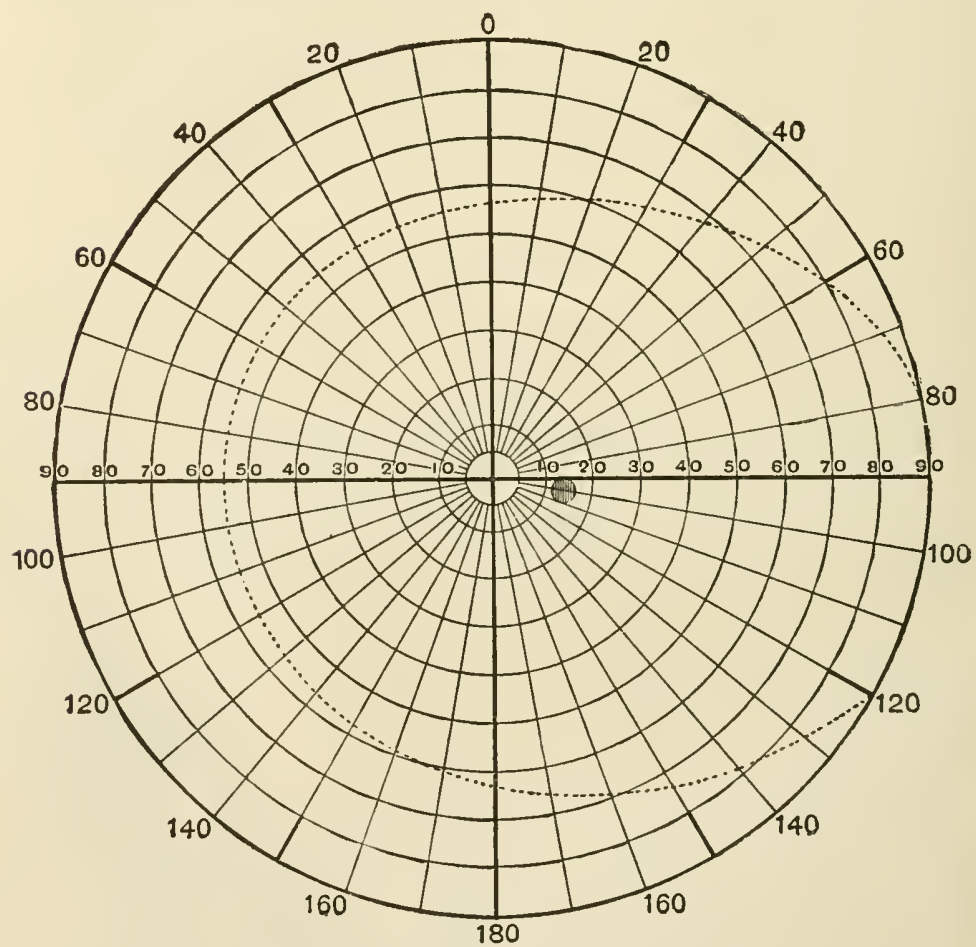

Fig. 177.-A Permetric Chart for the Right Eye. (Halliburton.)

to the outer than to the inner side, and further above than below the horizontal meridian.

In the case of colours it will be found that the capacity for distinguishing them diminishes more rapidly at the periphery than is the case for white light.

The field of vision is more extensive for blue than for other colours; it is least extensive for green and intermediate for red. 


\section{N D E X}

Absolute force of muscle, 52

Acceleration of heart by sympathetic, 126

Accomodation, 218

After-load, 42

Ammonia, excitation of muscle by, 78

Anelectrotonus, 88

Anodic contraction, 80

Anterior root of spinal nerve, function of, 86

Apex-time in isometric curves, 42

Artificial respiration apparatus, 138

Asphyxia, action of heart during, 144

- changes in blood-pressure during, 182

Atropine on frog's heart, 131

\section{Batteries, 1, 2, 3}

Beat of heart, record of, 98

Bernard's curare experiment, 76

Biedermann's fluid, 103

Blood-flow, velocity of, 188

Blood-pressure, arrangement of apparatus for, 168

- effect of asphyxia, 182

- nicotine, 181

- stimulation of depressor nerve, 175

_- of vagus, 177,186

- mean general, 182

-normal tracing, 172

Brain, behaviour of frog when its brain is destroyed, 214

Break, extra-current, 14, 19

Break-key, 30

Break-shock greater than make-shock, 14,18

Bunsen battery, 2

Caffeine citrate, action upon the heart, 143

- - - upon the kidney, 195

Cannula, arterial, 170

-- perfusion, 136
Capillary electrometer, 154

Cardiogram, 145

Cardiograph, Marey's, 144

Chemical stimulation of nerve, 86

Chorda tympani, dissection of, 211

- - stimulation of, 212

Chronogram, 22

Chronograph, 23

Circulation, schema of the, 157

Coil, induction, 4

Colour-blindness, 225

- - vision 224

Commutator, Pohl's, 12

Conductivity of nerve, changes during electrotonus, 90

Constant current, action on nerve, 87

- - excitation of muscle by, 79

Contact key, 10

Contraction, Law of, 93

- paradoxical, 156

- period, 33

- secondary, 148

- wave in muscle, 73

- with metals, 147

- without metals, 147

Contractur, 67

Convulsions, effect upon blood-pressure, 182

Crank lever, 26

Crescent in frog's heart, 98

- stimulation of, 125

C-spring manometer, 183

Curare, effect on muscle, 76

Current, constant, action on nerve, 87

- of action of muscle, 151

- of injury, 151

- of rest, 146

Cut-out key, 13

Cylinder, recording, 24

D.xiell battery, 1

Depressor nerve, action of, 175

- - anatomy of, 170

Dicrotic notch and wave, 165 
Digitalin, action on mammalian heart, 143

— - on kidney, 198

Direct excitation, 18

- vision, 225

Du Bois-Reymond's key, 11

Dudgeon's sphygmograph, 166

Dyspnœa, effect upon blood-pressure, 182

Electrical stimulation of nerve, 87

Electrodes, polarisation of, 81

— simple flexible, 106

- - wire, 26

- unpolarisable, 83

Electrometer, capillary, 154

Electro-physiology, 146

Electrotonus, 88

Engelmann's experiment, 81

Excitability, changes of, in electrotonus, 88

- independent muscular, 76

Excitation, direct and indirect, 18

- maximal and minimal, 26

- polar, of muscle, 80

— - of nerve, 90

- unipolar, 28

Extra-current, causation of, 6

— break, 14, 19

- make, 19

Fatigue of muscle, changes in height of twitch, 69

- - study of, 66

Fick's manometer, 183

Field of vision for colour, 227

Force, absolute, of muscle, 52

- of heart-beat, decrease of, by vagus, 122

- - increase of, by sympathetic,

Forceps, muscle, 25

Friction-key, 11

Galvani's experiment, contraction witl metals, 147

_ _ - without metals, 147

Galvanometer, 149

Gastrocnemius preparation, 16

Genesis of tetanus, 59

Glosso-pharyngea], action on respiration, 203

Gracilis and semimembranosus preparation, 19

Graphic method, 21

Green blindness, 225

Grenet battery, 2

Grove battery, 3
Heart (frog's), anatomy of, 97

- - action of atropine on, 131

- _. - muscarine on, 130

- _ - nicotine on, 133

- - - pilocarpine on, 131

- - - temperature, 112

- - currents of, 153

- current of action of, 149

- - excision of, 101

- Luciani's groups, 137

- minimal stimulns canses maximal contraction, 105

- nerves of, 120

- plethysmographic record of, 135

- rate of beat, 98

- record of beat, 98

— rhythm of, 102, 103

- stimulation of crescent, 125

Heart (mammalian), action during asphyxia, 144

- — of caffeine on, 143

- - o of digitalin on, 143

- - of neurine on, 144

— - of vagus on, 141

- recording movements of, 140

Helmholtz modification, 9

Helmholtz's phakoscope, 219

Holmgren's worsteds, 224

Hürthle's manometer, 184

Hyoglossus preparation, 20

InAGES formed by reflection from curved surfaces, 218

Independent muscular excitability, 76

Inertia, effect of, on single twitch, 37

Impulse, nervous, velocity of, 94

Indirect excitation, 18

- vision, 225

Induction coil, 4

Inhibition of frog's heart by vagas, 123

- of heart by muscarine, 130

- of reflexes, 217

Injection, intravenous, 181

Injury, current of, 151

Irradiation of reflex movements, 216

Irritability, independent muscular, 77

Isometric contraction, 39

- lever, 40

- muscle-curves, 41

Isotonic contraction, 39

KatelectrotoNus, 88

Kathodic contraction, 80

Key, break, 30

- cut-out, 13

- du Bois, 11

- friction, 11 
Key, mercury, 10

一 spring, 10

- trigger, 54

Kidney, course of vaso-motor nerves to, 200

Kidney-volume, action of caffeine on, 195

_ — of digitalin on, 198

- - of neurine on, 198

- normal tracing, 194

Kronecker's frog-heart manometer, 137

Kühne's curare experiment, 78

Kühne's experiment of contraction without metals, 147

- sartorius experiment, 77

LARYNGEAL, superior, action on respiration, 202

Latent period of muscle contraction, 33 - - of reflex action, 216

Law of contraction, 93

Leclanché battery, 3

Leg-muscles of frog, 17

Lever, crank, 26

- simple, 25

Load, influence of, on single twitch, 48

Luciani's groups, 137

Ludwig's stromuhr, 187

MAKE, extra-current, 6,19

shock less than break-shock, 14, 18

Manometer, 158

- Fick's, 183

- Hürthle's, 184

- Kronecker's frog-heart, 137

- mercury, 171

Marey's pneumograph, 206

- sphygmograph, 164

- tambour, 28

Maximal excitation, 26

Mechanical stimulation of nerve, 86

Mercury key, 10

Minimal excitation, 26

Moist chamber, 18

Monochord, 91,92

Muscarine, action on frog's heart, 130

Iuscle, excitation by constant current, 79

-- fatigue of, 66

- forceps, 25

- iso-electric when normal, 147

- less excitable than nerve, 77

— of frog's leg, 17

- simple curve, 34

- thickening of, during a twitch, 71

- wave of contraction, 73

- - rate of, 75

Muscular excitability, independent, 76
NEEF's hammer, 8

Negative variation, 146

- nature of, 148

Nerve, action of constant current on, 87

- changes of conductivity during electrotonus, 89

- - of excitability during electrotonus, 88

- forms of stimuli for, 86

- functions of, 85

- more excitable than muscle, 77

Nerve-muscle preparation, 16

Nerve-cells, submaxillary, paralysed by nicotine, 212

Nerves of rabbit's neck, 170

— to frog's heart, 120

Nervous impulse, velocity of, 94

Neurine, action on kidney, 198

- on mammalian heart, $14 t$

Nicotine, action on frog's heart, 133

-- - on submaxillary gland, 212

- effect on blood-pressure, 181

Non-polarisable electrodes, 83

ONCOGRAPH, 191

Oncometer, Roy's, 189

- air, 191

Ophthalmoscope, 222

Paranoxical contraction, 156

Pendulum myograph, 53

Perfusion cannula for frog's heart, 136

l'erimeter, 225

Period, latent, 33

- of contraction, 33

- of relaxation, 33

Pftïger's law, 93

Phakoscope, 219

Pilocarpine on frog's heart, 131

Pithing a frog, 16

Plethysmograph, Schäfer's frog-heart 136

Plethysmographic method for frog's heart, 135

Pneumograph, Marey's, '206;

Pohl's commutator, 12

Polar excitation of muscle, 80

— of nerve, 90

- Biedermann's method, 81

- Engelmann's method, 81

Polarisation of electrodes, 81

Posterior root of spinal nerve, function of, 85

Potential, measurement of, 152

Pressor effect, 176

Pressure bottle for manometer, 171

Primary coil, 4

Pulse-wave as studied on the schema, 162 
Pulse-wave in man, 165

Purkinje-Sanson images, 219

RATE of heart-beat, 98

- of muscle-wave, 75

- of work in simple twitch, 35

Recording cylinder, 24

- tambour, 28

- time, 22

Red-blindness, 225

Reed, vibrating, 60

Reflex action, 214

Reflexes, inhibition of, 217

- irradiation of, 216

- purposive character of, 216

Reflex time, 216

Refractory period of heart, 108

Relaxation period, 33

Respiration, action of central end of vagus, 206

- action of the glossopharyngeal, 203

- effect of swallowing upon, 203, 208

- methods of recording, 201

- result of section of the two vagi, 203

Respiratory movements, effect on bloodpressure, 173

Rheochord, 92

Rhythm of heart, 102, 103

- - effect of heat upon, 113

Ritter's tetanus, 87

Roots of nerve, functions of, 85

Salivary gland, secretory nerves to, 212

- secretion, 209

Sanderson's stethometer, 207

Sanson-Purkinje images, 219

Scheiner's experiment, 220

Schema of the circulation, 157

- - action of elastic tube, 161

Sciatic nerve, effect of stimulation of, upon blood-pressure, 175

Secondary coil, 4

- contraction, 148

Secretion of saliva, 209

Semimembranosus and gracilis prepara. tion, 19

Shunt of galvanometer, 150

Slowing of frog's heart by vagus, 123

Sphygmogram, 165167

Sphygmograph, Dudgeon's, 166

- Marey's, 164

Spinal cord, study of reflex action in 214

Spring chronograph, 24

- key, 10

Staircase-effect, in heart, 105

_- in muscle, 63,69

Stannius ligature, the first, 104

- - the second, 128
Stethogram, 208

Stethometer, Sanderson's, 207

Stimulation, unipolar, 28

Stimuli for nerve, 86

Stromuhr, 187

Strychnine, action on spinal cord, 217

Submaxillary gland, secretion by the, 203

Submaximal excitation, 27

Summation of muscular contraction, 56

Superior laryngeal, action of, upon the respiration, 202

Supra-renal extract, effect upon blood. pressure, 182

Suspension method of recording heartbeat, 98

Swallowing, effect of, upon respiration, 203,208

Sympathetic, action on heart, 125, 126

-_ - on submaxillary gland, 212

- course of fibres to frog's heart, 121

TAयBour, 28, 29

Temperature, influence of, upon a single muscle-twitch, 44

- - - a single beat of the heart, 116

- _ - upon the frog's heart, 112

Tetanisation of frog's ventricle, 110

Tetanus, complete, 64

- fatigue during, 70

- genesis of, 59

- Ritter's, 87

Thermal stimulation of nerve, 86

Thickening of muscle during a twitch, 71

Time, measurement of, 22

Tonometer, Roy's, 135

Trigger-key, 54

Twiteh, simple, 30

- thickening of muscle during a, 71

- modified by fatigue, 67

UNipolar excitation, 28

Unpolarisable electrodes, 83

VAGUs, action of eentral end upon respiration, 206

- - on heart, frog's, 122

- - - mammalian, 141

- course of, to frog's heart, 120

- effect of section upon respiration, 203

- - of stimulation upon blood-pres. sure, 177

- inhibition, escape of heart from, 179

Varnish for tracings, 32 
Vaso-motor nerves to kilney, 200

Velocity of nervous impulse, 94 of the blood-flow, 188

Ventricle contraction, 107

- - effect of heat, 116

- - - of tetanisation, 110

- - of two stimuli, 109

Veratrine, influence of, on muscle, 49
Vibrating reed, 60

Vision, direct and indirect, 225

WAVE of contraction in muscle, 73

- pulse, studied on schema, 162

Work performed during a twitch, 34,50

- in simple twitch, rate of, 35

- variation in amount with different loads, 51

PIRINTED BY

SPOTIISWUODE AND CO., NEW-STLRET SQUARE,

I.ON1) 








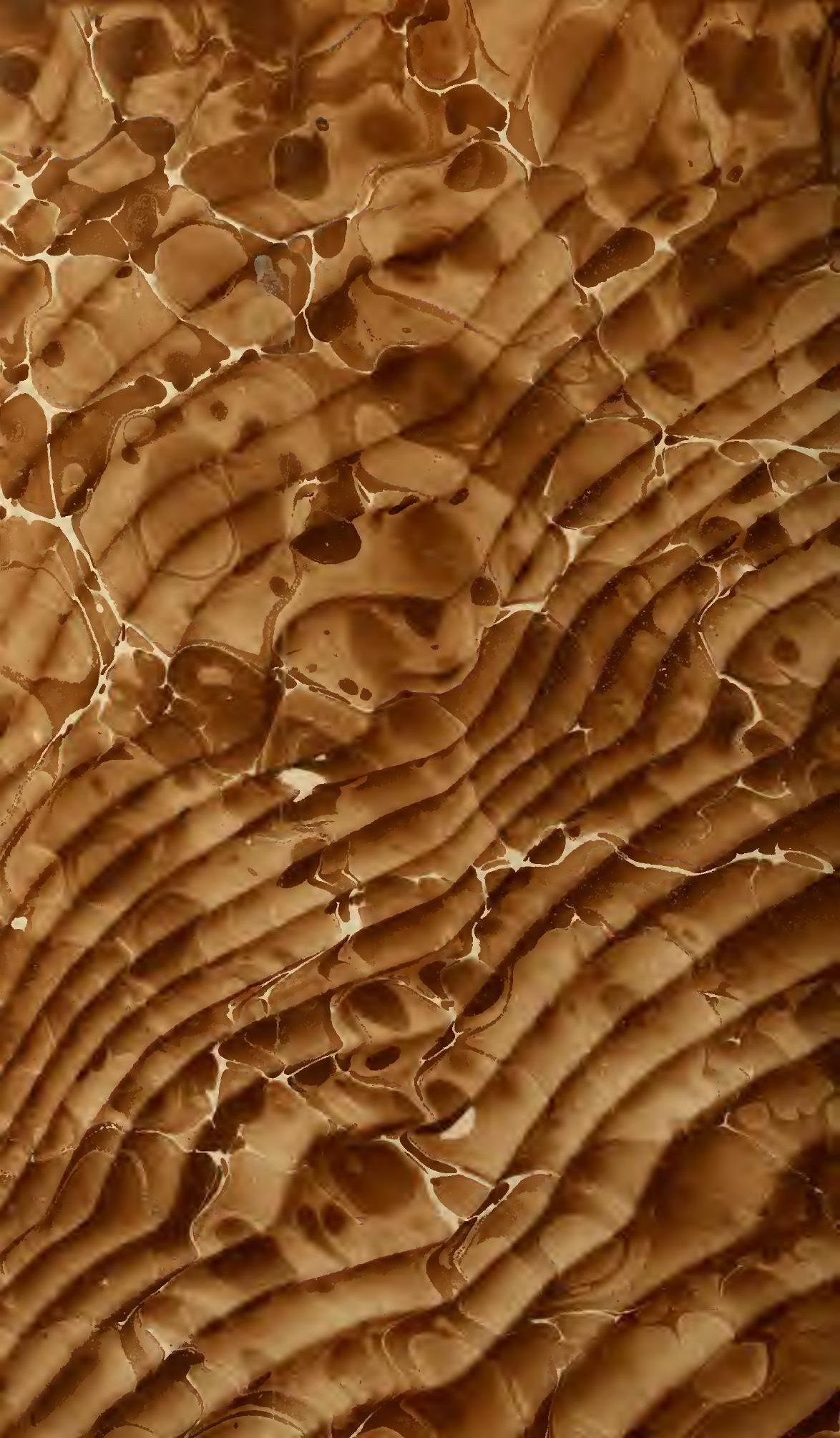




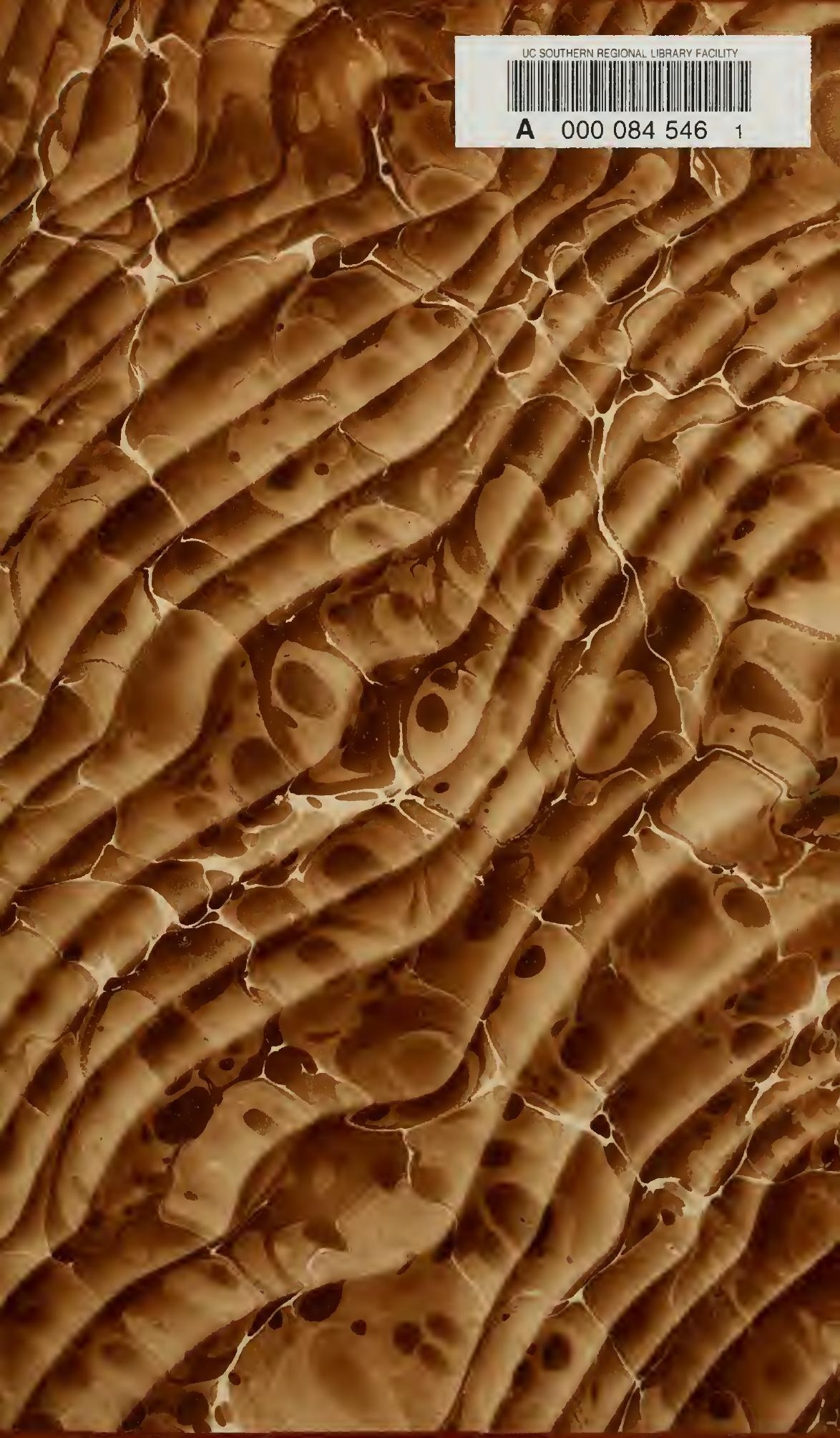




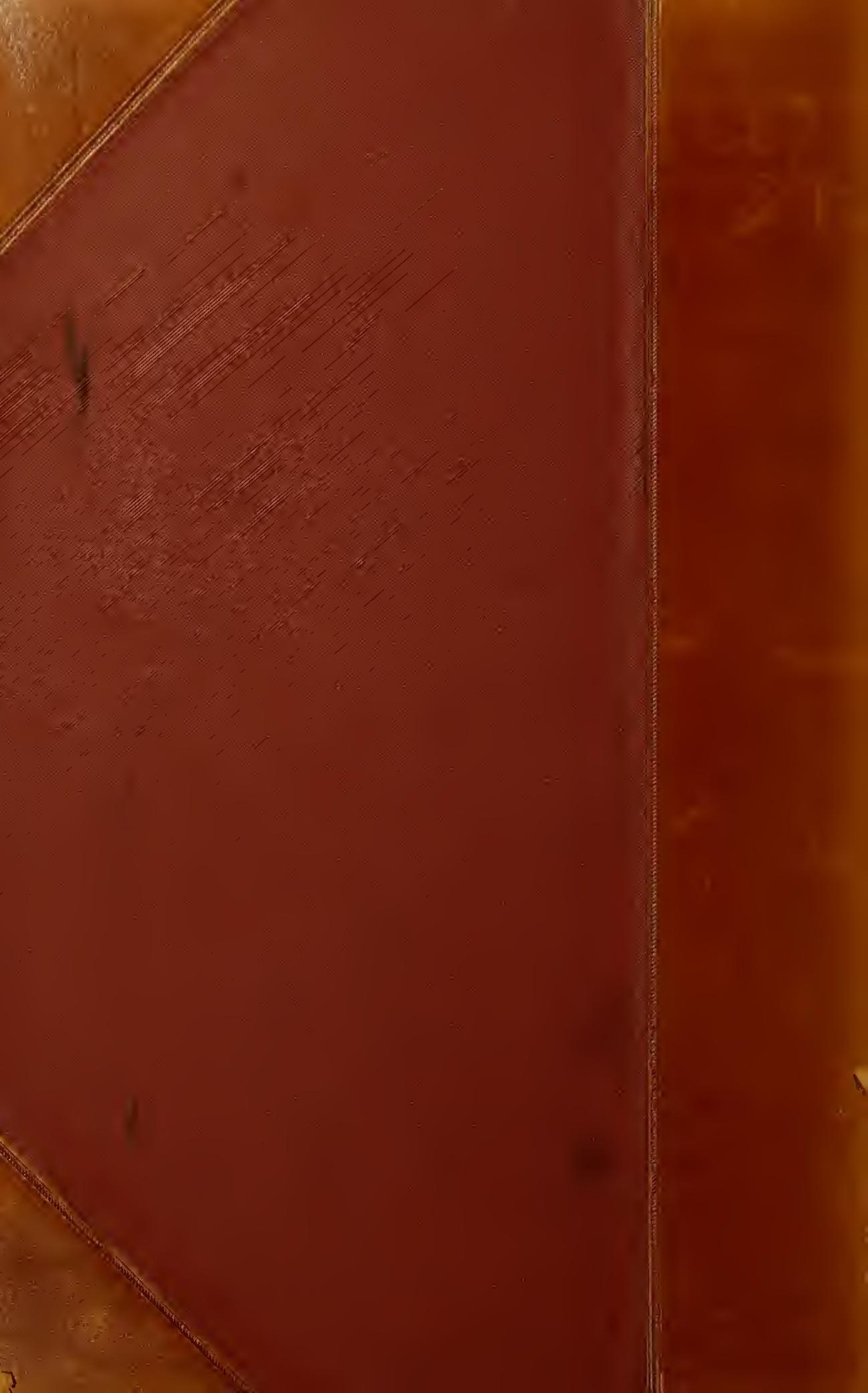

$4 \% ?$

.

18

1.

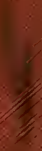

7 